\title{
The mechanics of soft porous solids: \\ from hydrogel dynamics to fibrin compression
}

Melle Punter 


\section{Propositions}

1. From volume measurements on the (de)swelling dynamics of synthetic hydrogels in polymer solutions, the poromechanical properties of the hydrogel network and the diffusivity of the polymers within the hydrogel can be inferred.

(this thesis)

2. The poromechanical properties of soft (bio)polymer networks bonded to the plates of a commercial rheometer can be inferred from force measurements in a single ramp compression test. (this thesis)

3. When the professional practice of a scientific discipline becomes engaged in political activism based on its own scientific findings, then this practice cannot credibly claim to strive for objectivity and thereby robs itself of its scientific prestige.

4. Although the materialistic approach to science has yielded an impressive amount of scientific knowledge, it cannot be concluded that this approach is the sole source of truths about reality.

5. Without a conscious exercise of the virtue of temperance, a society will find itself fighting fat-rich food instead of gluttony.

6. When a society does not acknowledge the existence of timeless truths, then this society is bound to have a brief lifetime.

Propositions belonging to the thesis, entitled

The mechanics of soft porous solids: from hydrogel dynamics to fibrin compression

Melle Tijmen Punter

Wageningen, $3^{\text {rd }}$ of Februari 2020 


\section{The mechanics of soft porous solids: \\ from hydrogel dynamics to fibrin compression}

Melle Tijmen Punter 


\section{Thesis committee}

\section{Promotor}

Prof. Dr B.M. Mulder

Professor of Theoretical Cell Physics

Wageningen University \& Research

Group leader Theory of Biomolecular Matter

AMOLF, Amsterdam

\section{Other members}

Prof. Dr E. (Erik) van der Linden, Wageningen University \& Research

Prof. Dr D. (Daniel) Bonn, University of Amsterdam

Prof. Dr E. (Erik) van der Giessen, University of Groningen

Prof. Dr K.P. (Krassimir) Velikov, Unilever, University of Amsterdam

This research was conducted under the auspices of the Graduate School VLAG

Advanced studies in Food Technology, Agrobiotechnology, Nutrition and Health Sciences 


\title{
The mechanics of soft porous solids: from hydrogel dynamics to fibrin compression
}

\author{
Melle Tijmen Punter
}

\section{Thesis}

submitted in fulfilment of the requirements for the degree of doctor at Wageningen University

by the authority of the Rector Magnificus

Prof. Dr A.P.J. Mol,

in the presence of the

Thesis Committee appointed by the Academic Board

to be defended in public

on Monday 3 Februari 2020

at 1:30 p.m. in the Aula. 


\section{Melle Tijmen Punter}

The mechanics of soft porous solids: from hydrogel dynamics to fibrin compression 222 pages.

$\mathrm{PhD}$ thesis, Wageningen University, Wageningen, The Netherlands (2020)

With references, with summaries in Dutch \& English

ISBN 978-94-92323-35-4

DOI https://doi.org/10.18174/511147 
Deo gratias 


\section{Contents}

Page

Chapter 1 Introduction 1

1.1 The history of mechanics: a topical and concise overview . . . . . . 2

1.2 Hydrogels . . . . . . . . . . . . . . . . . . . . 17

1.3 Biomechanics and fibrin fibers . . . . . . . . . . . . . . 19

1.4 Porous matter in industry . . . . . . . . . . . . . . . 23

1.5 Thesis outline . . . . . . . . . . . . . . . . . . 24

Chapter 2 The swelling and compression of hydrogels in concentrated $\begin{array}{ll}\text { polymer solutions } & 27\end{array}$

2.1 Introduction . . . . . . . . . . . . . . . . . . . . . . 29

2.2 Swelling and compression experiments . . . . . . . . . 30

2.3 Relaxational dynamics model . . . . . . . . . . . . . . . 33

2.4 Poroelastic model . . . . . . . . . . . . . . . . . . 46

2.5 Conclusions and discussion . . . . . . . . . . . . . . 60

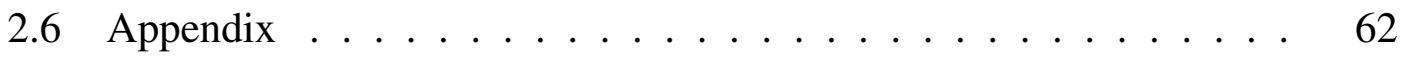

Chapter 3 Poroelasticity of (bio)polymer networks during compression: $\begin{array}{ll}\text { theory and experiment } & 90\end{array}$

3.1 Introduction . . . . . . . . . . . . . . . . . . . . 92

3.2 Materials and Methods . . . . . . . . . . . . . . . 96

3.3 Theoretical framework . . . . . . . . . . . . . . . . . 97

3.4 Results . . . . . . . . . . . . . . . . . . . . . . . 101

3.5 Conclusions and discussion . . . . . . . . . . . . . . 106

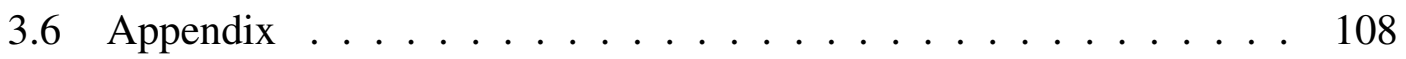


Chapter 4 Plate-sphere compression 136

4.1 Introduction . . . . . . . . . . . . . . . . . . . 138

4.2 Perturbation expansion around an incompressible solid . . . . . . . 140

4.3 Plate-sphere compression . . . . . . . . . . . . . . . . . . . 143

4.4 Conclusions and discussion . . . . . . . . . . . . . . . 147

4.5 Appendix ....................... 148

Chapter 5 Scoop syneresis $\quad 152$

5.1 Introduction . . . . . . . . . . . . . . . . 154

5.2 Syneresis experiments: materials, methods and results . . . . . . . 154

5.3 Two-cylinders model . . . . . . . . . . . . . . . 158

5.4 Filter paper clogging . . . . . . . . . . . . 159

5.5 Validation experiments .............. 171

5.6 Double network . . . . . . . . . . . . . . . . 174

5.7 Conclusions and discussion . . . . . . . . . . . . . . 177

$\begin{array}{lll}\text { Chapter } 6 & \text { General discussion } & 179\end{array}$

6.1 Hydrogel dynamics in polymer solutions . . . . . . . . . . . . . 180

6.2 Compression of (bio)polymer gels . . . . . . . . . . . . . . 182

6.3 Plate-sphere compression . . . . . . . . . . . . 185

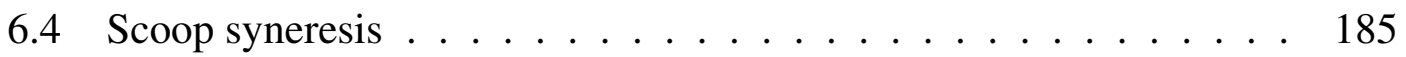

$\begin{array}{ll}\text { Appendices } & 188\end{array}$

$\begin{array}{lll}\text { Chapter A } & \text { Dimensonal analysis } & 188\end{array}$

A.1 Complete physical relations . . . . . . . . . . . . . 188

A.2 A frictionless pendulum . . . . . . . . . . . . . . . . 189

A.3 The $\Pi$-theorem . . . . . . . . . . . . . . . . . 191

A.4 A pendulum with friction . . . . . . . . . . . . . 192

$\begin{array}{ll}\text { Samenvatting } & 193\end{array}$

$\begin{array}{ll}\text { Summary } & 197\end{array}$

$\begin{array}{ll}\text { Acknowledgements } & 199\end{array}$

$\begin{array}{ll}\text { References } & 200\end{array}$ 
Chapter 1

\section{Introduction}


It is a privilege to be in a position to study the mechanical aspects of porous structures, because it is a rich and vast topic. Probably, the most common porous structure we encounter in daily life is the soil on which we stand. The soil provides the basis for all buildings due to its mechanical rigidity. At the same time, due to its porosity it provides both a home for the soil fauna and it absorbs rainfall, thereby allowing crops to grow. Within the home, we find porous water beads for decoration and a porous sponge to clean. When a dry water bead is put into water, it typically takes hours for the bead to swell. For a much larger sponge put in water, on the other hand, it can take only seconds to be filled. This difference in penetration time depends on the pore size and the elasticity of the materials. The water bead consists of a polymer network with tiny pores that cause a relatively low permeability, that is, the ease with which fluid can travel through a porous material. Moreover, with increasing elastic modulus of the polymer network, that is, the resistance associated to deformation of the network, the swelling time of a water bead decreases. The permeability and the elastic modulus are important examples of the poromechanical properties of materials. In this thesis, we mainly focus on the development of theoretical models which allow for the experimental determination of these properties. In particular, using the theory of poromechanics we model the dynamics of soft porous solids.

As we focus on the mechanical aspects of porous materials, we start the introduction by giving a topical and concise overview of the history of mechanics, and we pay special attention to the history of the theory of porous media. After sketching the historical background, we cover the scientific origin and the applications of the materials on which our theoretical models have been tested: synthetic hydrogels and fibrin gels. We end the introduction by providing an outline of the following research chapters.

\subsection{The history of mechanics: a topical and concise overview}

To situate this thesis in the whole of the body of scientific knowledge, we give an overview of some of the historical developments which led to the main theoretical framework which has been applied in this thesis: the theory of linear poroelasticity. For the sake of brevity and relevance, we mainly focus on two important lines which 
run within the voluminous history of mechanics; lines which lead from the ancient origins of mechanics to the modern theory of poroelasticity. The first line is the development of the concept of impetus, the assertion that a moving object actually contains a certain quantity of movement in itself, instead of the idea that the movement of the thing is perpetuated by its surroundings. The first line leads to the modern concepts of inertia, linear momentum and (kinetic) energy: foundations of the modern theory of poroelasticity. Second, we follow the evolution of the theories on the static equilibrium of objects, which is historically closely connected to the principle of virtual work or velocities, and can be illustrated by the classical lever problem: the determination of the equilibrium configuration of, for example, a balance. Treatments of this problem have been preparatory for modern mechanics, culminating in the conservation of angular momentum. Early work on the lever problem contained the germ for the principle of virtual work: an extremely flexible and versatile minimization principle which nowadays is still used to derive the momentum balance for continuous bodies, a foundation of continuum mechanics $[1,2]$.

\subsubsection{Origins of the science of mechanics}

Modern textbooks in physics, of which mechanics is a major subdiscipline, tend to give practically no coverage of the history of mechanics [3, 4], and neither do most doctoral theses. With this historical overview we deviate slightly from this tradition and try to give a not too folklorish account of the history of mechanics [5, p. 53], where we draw upon the work of historians of science. Indeed, historical awareness helps one to understand why science is what it is in the present day, and the knowledge of history allows one to appreciate and to scrutinize modern science, both in its scientific and in its social aspects.

At the start of this historical overview, one of the founding fathers of science should be acknowledged: Aristotle [6] (384-322 BC). Aristotle founded the famous Lyceum in Athens and he was a giant in natural philosophy whose thinking is relevant even today [7] in, for example, the philosophical interpretation of quantum mechanics [8]. In particular, we'd like to expound a little on his physica.

Interesting conceptual parallels can be drawn between Aristotle's physics, that of Isaac Newton (1642-1727), and that of Albert Einstein (1879-1955). Aristotle distinguished between natural motion and violent motion. Natural motion is that of 
bodies when 'left to themselves', whereas violent motion is caused by an external agent, e.g., a rock is violently moved by someone who throws it. The place to which each body naturally moves is for Aristotle determined by the more or less heavy elements of earth, water, air and fire present in the body, or, in modern language, the density of the body. Natural motion occurs vertically and brings the body to its natural place, where it remains. Violent motion, on the other hand, displaces an object from its natural place: a stone is displaced by throwing it higher up on a mountain, for instance. In his turn, Newton considers the motion of a body left to itself to be a rectilinear uniform motion through space, that is to say, Newton's law of inertia specifies the 'natural motion' of the body. External agents, however, can cause a 'violent motion' by exerting a net force on a body, thereby causing it to accelerate and change its state of motion [9, pp. 27,28]. Aristotle's treatment of the dynamics of the place of a body seems analogous to Newton's treatment of the dynamics of the velocity of a body. For Aristotle, a natural motion leads to a definite place of a body which it keeps, whereas for Newton a body naturally keeps its velocity. For Aristotle a violent motion changes the place of the body whereas for Newton the velocity of the body is changed. This reflects a fundamental difference between the modern concept of motion and that of Aristotle [10, pp. 5-9]. Furthermore, Einstein altered Newton's concept of 'natural motion' by posing that without external forces a body moves on a geodesic in space-time, i.e., an object naturally retains its four velocity when moving freely through space-time.

As a side note, during Aristotle's reflections on the vacuum he already formulated the essence of Newton's first law: a body in vacuum will either stay at rest or will move indefinitely until it collides with something. In the vacuum, however, he supposed a body could not reach its natural place, causing Aristotle to regard the vacuum as an impossibility and his reflections to remain only a thought [11, p. 22].

In modern times, Aristotle's physics is often said to be simply incorrect or it is given very little credit [12]. The fact remains, however, that Aristotle's physics gave an account of the motion of objects in agreement with, and restricted to, the capabilities of common observation: it was an accurate qualitative account with a restricted range of validity [9, p. 24]. The qualitative nature of his treatment is not surprising, as the concepts he uses in his physics are not differentiated from concepts having a more general significance [11, p. 20]. In the words of Rovelli [9, p. 23]: Aristotelian physics, or more specifically Aristotle's theory of local movement, is to be considered 
as "...a correct and non-intuitive approximation of Newtonian physics in the suitable domain (motion in fluids) in the same technical sense in which Newton's theory is an approximation of Einstein's theory".

\section{Impetus}

For the concept of inertia, the historic thread runs all the way from Aristotle to Newton. Without a vacuum, Aristotle considered the (violent) motion of a body to be perpetuated by the surrounding air, after the object is released by the external agent that moves the body. The surrounding air was supposed to have an immaterial faculty which allowed the air to maintain the movement of the body. This supposition follows from the notion that air is naturally apt to be moved and to move other things. For example, air is easily set in motion and wind moves all other things [13, p. 125]. John of Alexandria, also known as Philoponus (5-6 ${ }^{\text {th }}$ century AD), is an early natural philosopher who is known to deviate from this explanation. He claimed that when an object is thrown it incorporates a certain power of self-movement, and that the medium through which the object moves hinders the object, rather than that the medium perpetuates the movement of the object [11, p. 47]. In this way he gave an explanation of motion of objects without an external cause, and, importantly, he applied these notions to both natural and violent motion, as well as terrestrial and celestial motion $[13$, p. 126]: the movement of objects on earth and the movement of celestial objects, e.g., the sun.

After Philoponus, this conception of motion was preserved by the Arabic philosophers, e.g., Avicenna. Perhaps it was through them that this concept arrived in the Latin West [14, p. 40], though this is not completely clear [13, p. 129]. In the Latin West, the first ones to formulate a clear conception of impetus were Buridan and Albert of Saxony (14 ${ }^{\text {th }}$ century AD) within the 'Parisian school' of natural philosophy. Buridan poses, similar to Philoponus, that air resists motion rather than furthering it, and he concludes that a projectile is moved by a 'force' which is impressed into it, the force being greater if the object is more massive [13, p. 134]. The following passage gives a good impression of Buridan's thoughts on motion

"Therefore, it seems to me it should be said that a motor in moving a body impresses in it a certain impetus or a certain motive power capable of moving this body in the direction in which the mover moves it, either 
up or down or laterally or circularly. By the same amount that the motor moves the same body more swiftly, by that same amount is more powerful the impetus which is impressed in it. And it is from this impetus that the stone is moved after that which hurls it ceases to move; but on account of the resisting air and the gravity of the stone which inclines it contrary to the way which the impetus has power to move it, the impetus is continually weakened. Therefore, the movement of the stone will become continually slower. At length this impetus is so diminished or destroyed that the gravity of the stone prevails over it and moves the stone down to its natural place... I can throw a stone farther than I can a feather, and iron or lead fitted to hand farther than a piece of wood of the same size. I say that the cause of this is that the reception of all forms and natural dispositions is in matter and by reason of matter. Therefore, the greater quantity of matter there is the more that body can receive of this impetus and the more intensely can it receive it. Now in a dense and heavy body there is more of first matter than in a rare and light one. Therefore, a dense and heavy body receives more of the impetus and does so more intensely... . A feather, moreover, receives such a weak impetus that immediately it is destroyed by the resistance of the air. And so likewise if one moves equally swiftly by hurling a light piece of wood and a heavy piece of iron of the same shape and volume, the iron will move farther because there is impressed in it a more intense impetus...". [14, pp. 40,41]

This passage clearly suggests that his impetus was a qualitative notion which comes close to the modern concepts of linear momentum [5, p. 56] and energy [15, p. 194]. Similar to Philoponus, Buridan and Albert of Saxony applied these concepts to both terrestrial and celestial motions [11, pp. 48-51], foreshadowing Newton who will do similarly three centuries later. The influence of Buridan and Albert allowed the concept of impetus to become a well known scholastic doctrine in the $16^{\text {th }}$ century $[13$, p. 138]. Subsequently, Galileo Galilei (1564-1642) went on to formulate a physics of impetus which is thought to be a logical successor of the medieval account of impetus [16, p. 27], even though Galilei dismissed the foundation of Aristotelian physics [13, pp. 140,141]. After Galilei, the concept of impetus lives on in the works of scholars like Joseph Moxon (1627-1691), for instance. But their use of the word impetus occurs in a rather different philosophical context than with the Scholastics. 
Gradually, the concept loses its Scholastic meaning and becomes something more like the modern day impulse [13, pp. 142,143], until Newton finally described the quantity of motion as momentum, the product of the inertial mass of the body and velocity [14, p. 40]. At the same time, Gottfried Leibniz (1646-1716) formulated a precursor to the modern notion of kinetic energy under the name vis motrix, although it was not yet clearly distinguished from linear momentum [11, pp. 219-221].

\section{The principle of virtual work}

The principle of virtual work is a very general principle that manifests itself in many different forms and flavours: the principle of virtual displacement, the principle of virtual velocities, etc. It has undergone quite some changes through history, culminating in the variational principles which are fundamental to modern theoretical physics [17]. Modern versions of the principle of virtual work are used to derive, for example, the force balance for a continuum body from the principle of virtual power [1, pp. 20,21].

Let us illustrate the principle of virtual work with the following example, which we describe in modern terms. Consider the rigid curved lever in Figure 1.1, hanging on the top $S$ of a frictionless pointed pole around which the lever can rotate. The lever consists of two connected massless rods of length $l_{1}$ and $l_{2}$, making a fixed acute angle of $\theta_{\mathrm{t}}$, with two objects of mass $m_{1}$ and $m_{2}$ being attached at the ends of the lever. The weight of the masses makes the lever rotate in opposite directions, thereby varying the angle $\theta_{1}$ between the lever and the vertical. Given the magnitude of the masses, the dimensions of the lever and the acute angle, one may ask at what angle $\theta_{1}$ the lever will rest in equilibrium. Using

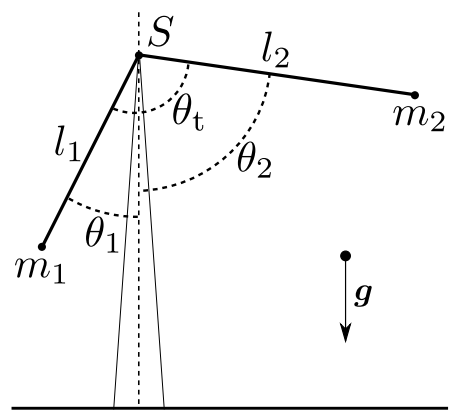

Figure 1.1: A curved rigid lever is supported by a pole at $S$. The lever consists of two massless rods of length $l_{1}$ and $l_{2}$, the acute angle between the rods $\theta_{t}$ being fixed, and has two objects of mass $m_{1}$ and $m_{2}$ attached at its ends. The gravitational acceleration $g$ pulls both masses downwards, causing the system to rotate around $S$. When the lever rests in equilibrium, what is the angle $\theta_{1}$ between the first rod and the vertical? the principle of virtual work, one can derive the condition that $\theta_{1}$ satisfies in equilibrium. 
We consider the work done by the gravitational force on the lever-mass complex when it undergoes a small virtual rotation around the angle $\theta_{1}$ which the lever makes with the vertical. The work which this virtual rotation produces, is called the virtual work performed on the system. The principle of virtual work states that, when the lever is in equilibrium, the virtual work must vanish for any small virtual rotation. This requirement provides a condition for the value of $\theta_{1}$ in equilibrium, and thus determines the rest configuration of the lever. Let the rotation change the angle $\theta_{1}$ by a value $\Delta \theta$. The work $\Delta W$ performed by the gravitational force due to the virtual rotation is thus given as

$$
\begin{aligned}
\Delta W & =\boldsymbol{F}_{1} \cdot \Delta \boldsymbol{x}_{1}+\boldsymbol{F}_{2} \cdot \Delta \boldsymbol{x}_{2}, \\
& =m_{1} g l_{1}\left(\cos \left(\theta_{1}+\Delta \theta\right)-\cos \left(\theta_{1}\right)\right)+m_{2} g l_{2}\left(\cos \left(\theta_{2}-\Delta \theta\right)-\cos \left(\theta_{2}\right)\right),
\end{aligned}
$$

where we defined $\theta_{2}=\theta_{\mathrm{t}}-\theta_{1}$, see Figure 1.1. $\boldsymbol{F}_{i}=m_{i} \boldsymbol{g}=-m_{i} g \hat{z}$ is the gravitational force, with $\hat{z}$ pointing in the positive vertical direction, and $\Delta \boldsymbol{x}_{i}$ is the displacement of a mass, with $i=1,2$. Expanding equation (1.1) as a Taylor series in $\Delta \theta$, and requiring the virtual work to vanish up to first order for any value of the variation $\Delta \theta$, we obtain the following condition

$$
m_{1} l_{1} \sin \left(\theta_{1}\right)=m_{2} l_{2} \sin \left(\theta_{2}\right)
$$

which is the well known statement that the moments of the forces acting on a body in static equilibrium cancel each other, namely, the law of the lever. This example shows explicitly that the 'equation of motion' in equation (1.2) can be found from the requirement that the virtual work vanishes at first order in the variation, the principle that is used in any variational technique up to this day.

The development of the principle of virtual work through history has centered itself around the lever problem [11]. Possibly, the most basic form of the principle can be found with Aristotle where the effect of a force is considered to be not only dependent on its magnitude, but also by the motion associated with this force [18, p. 44]. The first known to investigate the law of the lever quantitatively was Archimedes (287-212 BC). After him, Hero ( $2^{\text {nd }}$ century AD) derived the principle of virtual velocities from the law of the lever, although some historians say it is exactly the other way around [18, p. 56]; in any case it is contained in his work [18, p. 57]. Both in Arabic and Latin writers, one can find (implicit) virtual work principles. Most notably, Jordanus $\left(13^{\text {th }}\right.$ century AD) proved equation (1.2) for the case $\theta_{\mathrm{t}}=180^{\circ}[18$, 
p. 86], which is the simplest case of the law of the levers where equilibrium requires the ratio of the masses to be inversely proportional to the ratio of their distance to the turning point $S$, independently of $\theta_{1}$. He did so, however, without knowledge of modern concepts like mass, work and vectors, for instance. In this derivation, he used the principle gravitas secundum situm, which contained an implicit notion of the principle of virtual work [11, pp. 20,38,40,44,46].

Later, we see the principle of virtual work returning in Simon Stevin's (1548-1620) work on statics [11, p. 127], and the principle of virtual velocity in the work of Galilei (1564-1642) on hydrostatics [11, p. 143]. An important advance in the concept of virtual work was made by René Descartes (1596-1650), who stressed its infinitesimal character [11, p. 156]. The durability of this insight can be witnessed in the above example of the curved lever. With Newton (1642-1727), we do not see a clear incidence of the principle of virtual work, although he does seem to refer to a concept near to the modern concept of power [18, p. 194]. After Newton, the concept of virtual work is perpetuated in, among others, the works of John Bernoulli (1667-1748), this will be discussed in the following section.

\subsubsection{From continuum mechanics to the theory of poromechanics}

Poromechanics, the mechanical theory for porous bodies, is a subdiscipline of the broader field of continuum mechanics, including all materials from fluids to solids to gels. The basis for the theory of continuum mechanics was laid in the $18^{\text {th }}$ and $19^{\text {th }}$ century. The well known scientists of this time mostly held a corpuscular worldview: the world was thought to be made up of particles interacting via at-a-distance forces, it was a very Newtonian worldview. Not surprisingly, several attempts were made to derive the mechanical behaviour of a continuous body from a corpuscular theory, where the body transmits forces by contact instead of at-a-distance forces. Ironically, among all these endeavors an essentially phenomenological approach of Augustin-Louis Cauchy (1789-1857) has stood the test of time gloriously. Many other approaches were flawed, and caused 'the elastic constant controversy': the dispute on whether the number of elastic constants of an isotropic material is either one or two [19, pp. 216-218]. The number of elastic constants of an isotropic material turned out to be two and can be taken as, for instance, the couple of the shear modulus $G$ and the bulk modulus $K$, or as the longitudinal modulus $M$ and the Poisson 
ratio $\nu$, or as Lamé's first parameter $\Lambda$ and the Young's modulus $E$. These different constants quantify the response of a material to specific deformations. For example, the shear modulus determines the stress response to a shear deformation, the Young's modulus and the Poisson ratio give, respectively, the normal force and the degree of lateral expansion of a compressed material that is laterally unconstrained, while the longitudinal modulus gives the compressive normal force of a material that is laterally constrained.

Cauchy's phenomenological approach is widely used in applied mathematics and the engineering sciences up to this day, including this thesis. Presently, there exists no general corpuscular theory for continua, although for specific materials first-principle theories do exist, e.g., the statistical theory of liquid solutions [20, ch.1]. In the absence of a first-principles theory, one needs to postulate a so-called constitutive relation, peculiar to a specific material, to relate the Cauchy stress tensor to what causes stress in the material: the deformation gradient in a solid body, for instance. In general, this constitutive relation is not unique, although it can be constrained by certain principles, e.g., the principle of material frame-indifference [21, p. 8]. In spite of this non-uniqueness problem, for small deformations around the natural configuration of the material, the constitutive relation is linear. In chapter 2, 3 and 4 the material response of the solid (part of the) material is modelled as being linear elastic, which is by far the most investigated constitutive relationship.

The modern relevance of the theory of continuum mechanics can possibly be framed as a reappraisal of Aristotle's philosophy. Contrary to other Hellenistic philosophers, e.g., Heraclitus (540-480 BC), who fashioned an atomistic worldview, Aristotle (384-322 BC) was the first known to disregard the concepts of atoms and the void, and to foster the concept of a continuum instead [22, pp. 1,2]. Only in the time of Descartes did the concept of the atom resurface. This gave rise to the corpuscular worldview, especially through Newton, and resulted in a fruitful path of inquiry. As explained above, however, the continuum worldview has proven also to be highly fruitful. Roughly speaking, corpuscular approaches still dominate the field of physics, while continuum approaches dominate the field of engineering.

Below, we will describe in more detail the developments leading to the theory of poromechanics, also pointing out the connection with the classical concepts of impetus and the principle of virtual work. 
The $18^{\text {th }}$ and $19^{\text {th }}$ century

After Newton, scientists of the $18^{\text {th }}$ century started to study not only point particles, but also whole bodies [23, pp. 6,7], e.g., a body of water, air, wood, iron, etc. Here, the historical lines of impetus and the principle of virtual work converge. On the one hand, in the line of impetus these scientists performed fundamental work in fluid mechanics which was based on the conservation of linear and angular momentum. On the other hand, in the line of the principle of virtual work it developed variational principles. One key contributor to this movement was Leonhard Euler (1707-1783) who, probably inspired by Jean-Baptiste d'Alembert (1717-1783), introduced the notion of internal pressure. With this notion, and using Newton's principle of linear momentum, he was the first to write down the field equations of motion for a perfect fluid: a frictionless fluid [24, ch.2]. Later, as noted above, Cauchy generalized Euler's notion of pressure to the Cauchy stress tensor, which is used up to this day to specify the general state of stress in a continuum, both for fluids and solids [24, ch.3]. In modern notation, the balance of linear momentum per unit of volume in a body, that

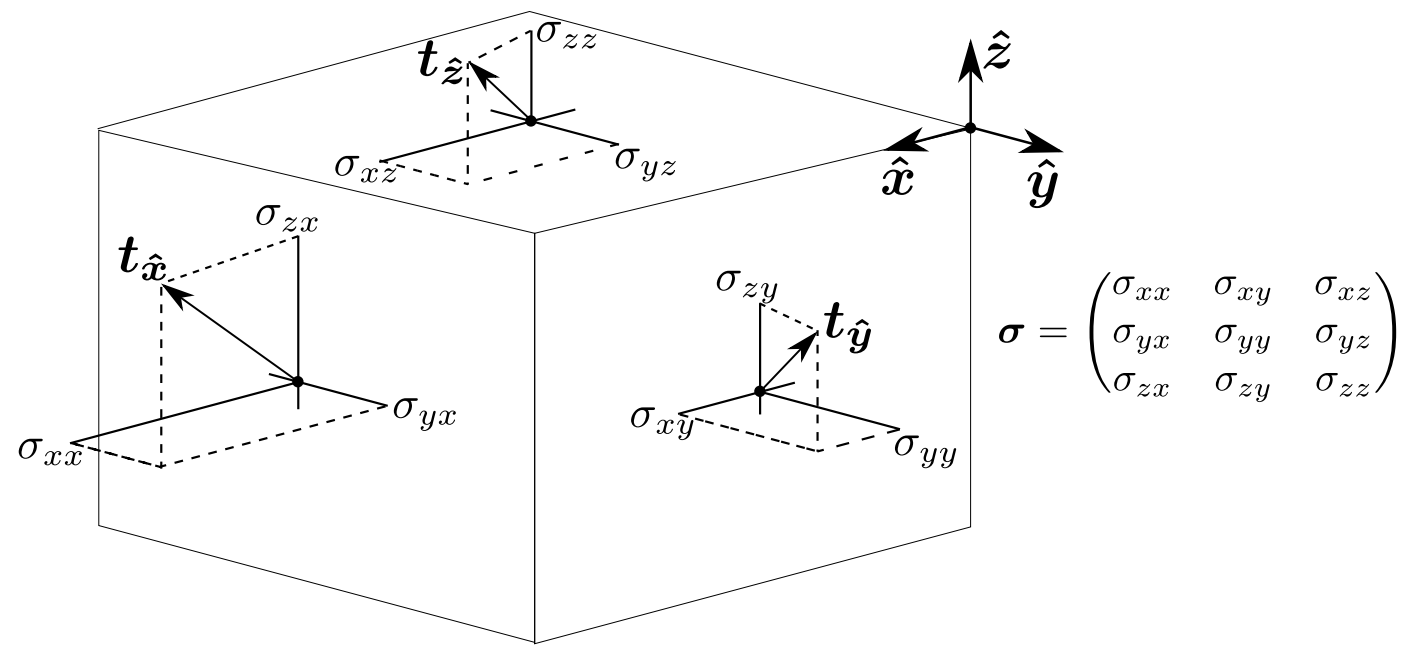

Figure 1.2: An infinitesimal cubic volume element in a material body where the Cauchy stress tensor $\sigma$ specifies the stresses acting on the faces of the cube. At each face the surroundings exert a traction $\boldsymbol{t}_{\hat{\boldsymbol{n}}}=\boldsymbol{\sigma}^{\mathrm{T}} \hat{\boldsymbol{n}}$, with $\hat{\boldsymbol{n}}$ the outward normal vector to the surface. As the Cauchy stress tensor is symmetric $\sigma=\sigma^{\mathrm{T}}$, the tractions do not exert a net torque on the cube. 
is, Newton's second law for a continuum, is written as

$$
\nabla \cdot \boldsymbol{\sigma}+\rho \boldsymbol{f}=\rho \frac{\partial^{2} \boldsymbol{U}}{\partial t^{2}}
$$

where $\boldsymbol{\sigma}$ is the Cauchy stress tensor, $\rho$ the mass density, $\boldsymbol{f}$ the external force per unit of mass, and $\boldsymbol{U}(\boldsymbol{x}, t)$ the displacement of a part of the body at position $\boldsymbol{x}$ at time $t$. The first term gives the resultant force density acting on an infinitesimal volume element from contact with the surrounding part of the body, the second accounts for all non-contact forces (gravity, electrical, etc.), and the right hand side is the force density required for acceleration. The balance of angular momentum implies that the Cauchy stress tensor is symmetric $\boldsymbol{\sigma}=\boldsymbol{\sigma}^{\mathrm{T}}$. By Cauchy's theorem, the force per unit area on any infinitesimal surface area in the body with normal vector $\hat{\boldsymbol{n}}$, namely, a traction, is expressed as $\boldsymbol{t}=\boldsymbol{\sigma}^{\mathrm{T}} \hat{\boldsymbol{n}}$, see Figure 1.2 for the tractions on an infinitesimal cubic volume element in Cartesian coordinates.

With the notion of the Cauchy stress tensor many important contributions could be made to the theory of continuum mechanics. Among others, Adhémar de SaintVenant (1797-1886) deserves mentioning, in particular for his introduction of SaintVenant's principle. This principle states that for a body with high aspect ratio(s) in its geometrical dimensions, e.g., a slender rod, it is not the exact force distribution applied to the ends of the rod which determines the deformation of the body 'far away' from the ends, but only the average force and the torque [24, ch.6]. This important principle will be used in chapter 3, where we consider a disk-like cylindrical gel compressed between two plates parallel to the ends of the cylinder, while the curved area of the cylinder remains free. Instead of requiring the radial and tangential stress at the free surface to vanish pointwise, we only require it to vanish when averaged over the free surface. In this way, we find a solution which is bound to fail near the free boundary, but is a good approximation far away from it - far away being a few times the axial length of the gel since this is the dimension over which we average the stress at the free boundary.

With regard to the porous nature of materials, in 1762 Euler was the first to speak about the pores of a material which would give rise to the elasticity of a material [20, pp. 57,58]. Although his treatment was far from satisfactory, he did make a distinction between 'rough' and 'subtile' matter in a body, which we now, respectively, identify as matrix and fluid [23, p. 24]. In modern terms, a porous material is defined as a material made of a deformable matrix/network/solid phase whose pores are filled 
with fluid, be it (any combination of) air, water or oil, for instance. Due to the connectedness of the pores of the material the fluid can flow through the matrix. A major step in the direction of poromechanical theory was the contribution of Darcy, with the famous law carrying his name. Before that, however, both Reinhard Woltman (17571837) and Achille Delesse (1817-1881) are to be credited with the introduction of the concept of a volume fraction: the discrimination between the volume of space which a porous solid $V_{\mathrm{s}}$ encompasses and the volume that the interpenetrating fluid $V_{\mathrm{f}}$ occupies, summing up to the total volume $V_{\mathrm{t}}=V_{\mathrm{s}}+V_{\mathrm{f}}$ of a volume element in the porous material, see Figure 1.3 for an example. This gives the volume fraction of the solid and the fluid as $\phi_{i}=V_{i} / V_{\mathrm{t}}$, with $1=\phi_{\mathrm{s}}+\phi_{\mathrm{f}}$. Importantly, Delesse found the volume fraction to be equal to the area fraction of the two phases, the area fraction being defined as the ratio of the area of solid or fluid to the total area of a plane cut of the porous material [25, p. 213]. Besides the concept of volume fraction, the introductions of viscosity by Jean Poiseuille (1797-1869) and Claude-Louis Navier (1785-1856) were important precursors to Darcy, because it quantified the resistance for fluid to flow through a tube [20, pp. 58,59], which is closely related to the flow through a porous material. In fact, as a first approximation one can consider a porous material as a collection of parallel tubes [26].

Henry Darcy (1803-1856), being born in Dijon, investigated the supply systems of drinkable water to the cities, in particular he studied this in his own hometown [20, pp. 59,60]. In this work Darcy proposed a law for laminar fluid flow through a porous medium that came to be known as Darcy's law, and that reads in modern form [27]

$$
\phi_{\mathrm{f}}\left(\boldsymbol{v}_{\mathrm{f}}-\boldsymbol{v}_{\mathrm{s}}\right)=-\frac{k}{\eta}\left(\nabla p-\rho_{\mathrm{f}} \boldsymbol{g}\right),
$$

with $\boldsymbol{v}_{i}$ the local average velocity of the solid or fluid, $k$ the permeability of the porous solid, $\eta$ the dynamic viscosity of the fluid, $p$ the fluid pressure, $\rho_{\mathrm{f}}$ the mass density of the fluid and $\boldsymbol{g}$ the gravitational acceleration. It proposes the average flow of fluid relative to the average velocity of the solid to be simply proportional to the local gradient of the fluid pressure corrected for the hydrostatic pressure, that is, it is proportional to the fluid overpressure. Equation (1.4) can be regarded as a linear response relation between the fluid mass flux and the fluid pressure for a porous material. In modern approaches, Darcy's law can be derived with a nonequilibrium thermodynamics approach [28, ch.3], or, if the fluid-filled pore itself can be considered as a continuum, by averaging the laminar flow of a Newtonian fluid 
through the pores over a volume called the representative volume element [20, ch.3]. The dimension of this volume element $l$ should typically be an order of magnitude larger than the dimension of both the fluid-filled pores and the solid phase. At the same time, the size of the volume element should be small compared to the macroscopic size of the porous body $L$, such that $(l / L)^{2} \ll 1$ [30]. The concept of a representative volume element also allows to properly define the concept of a volume fraction, see Figure 1.3. Currently, ongoing research is pursued to extent Darcy's law for inertial effects in the fluid flow, and for rarefied gasses [31]. Interestingly, one year before Darcy formulated his law, Fick experimentally discovered another linear response relationship describing the mass flux of dissolved particles due to a concentration gradient. Fick's law was in turn inspired by Fourier's law of heat conduction, where the heat flux also satisfies a linear response relationship [23, pp. 97-99]. All three laws, Darcy's,

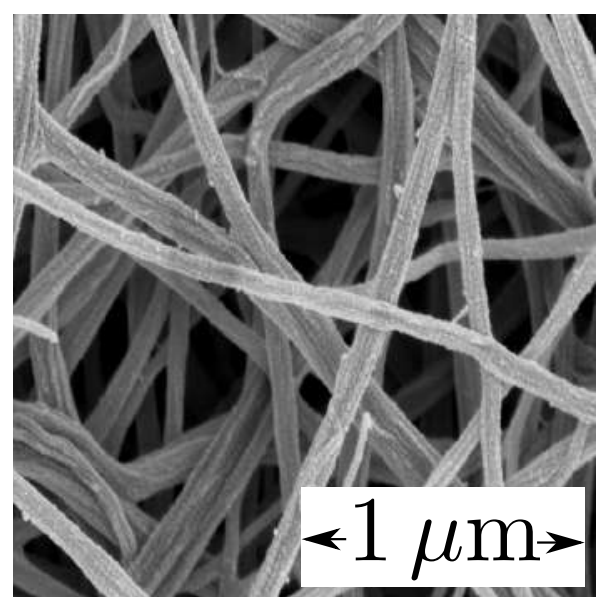

Figure 1.3: Scanning electron microscopy image of a fibrin fiber network (the structural component of blood clots, see section 1.3.1). Consider a volume element of total volume $V_{\mathrm{t}}$ where the fluid and the network encompass, respectively, a volume $V_{\mathrm{f}}$ and $V_{\mathrm{n}}$, with $V_{\mathrm{t}}=V_{\mathrm{f}}+V_{\mathrm{n}}$. For a representative volume element of linear dimension $l$, which is much smaller than the macroscopic size of the network $L$ but much larger than the pore size, i.e., $1 \mu \mathrm{m} \ll l \ll L$, the local volume fraction of fluid and network in the porous material is given as $\phi_{i}=V_{i} / V_{\mathrm{t}}$. Scanning electron microscopy image taken from [29].

Fick's and Fourier's, have proven to be fruitful theoretical assumptions and are used extensively up until today.

Finally, the first to pioneer a more theoretical continuum mechanics approach to poromechanics was Josef Stefan (1835-1893). He investigated mixtures of three components and was the first to employ herein the concept of volume fraction [25, p. 215]. Many other contributions deserve mentioning, but for this thesis we focus, for example, not on temperature-dependent effects, so we ignore those otherwise relevant contributions. In all investigations we assume the temperature throughout the porous body to be constant, since either the thermal diffusion time scale is much smaller than the gel dynamics time scale (chapter 2 and 5), or temperature related 
effects are expected to be small (chapter 3). Moreover, the general thermodynamical theory of mixtures still poses open questions [20, p. 65], making it a story on its own.

\section{From the $20^{\text {th }}$ century to the present day}

With these foundations of poromechanical theory from the nineteenth century in mind, we arrive at a period characterized by geomechanics, i.e., the study of the mechanics of soil and rocks, starting at the beginning of the $20^{\text {th }}$ century, after which the modern theory of poromechanics was developed. In the $20^{\text {th }}$ century, one can roughly distinguish between two main schools of thought: one is more empirically oriented and founded by Karl von Terzaghi (1883-1963), whilst the other is more theoretically oriented, with Paul Fillunger (1883-1937) as its first notable adherent. Terzaghi and Fillunger were both professors at the technical university of Vienna, and they got caught up in a scientific and personal conflict which ended with the woeful suicide of Fillunger [23]. This tragic event probably also hampered the more theoretical school of thought in geomechanics [25, p. 218].

A major contribution from Terzaghi is where he split the Cauchy stress tensor of a porous material in two parts: the pressure of the fluid and an additional stress due to the solid, the Terzaghi effective stress. The rationale behind this distinction is that the solid imbibes the fluid and therefore it inherits the pressure of the fluid, causing a nonzero stress in the solid even when it is not strained, that is, when it is in its stress-free conformation. When correcting the solid stress for the fluid pressure, one finds the stress in the solid due to, for example, strain in the solid material. Considering a porous material as the superposition of a solid and a fluid continuum with, respectively, Cauchy stress tensor $\boldsymbol{\sigma}_{\mathrm{s}}$ and fluid stress tensor $\boldsymbol{\sigma}_{\mathrm{f}}=-p \mathbb{1}$, the Terzaghi effective stress is defined as $\sigma^{\prime}=\phi_{\mathrm{s}}\left(\boldsymbol{\sigma}_{\mathrm{s}}-\boldsymbol{\sigma}_{\mathrm{f}}\right)$, giving the Cauchy stress tensor of the porous material $\sigma$ as [27]

$$
\boldsymbol{\sigma}=\boldsymbol{\sigma}^{\prime}-p \mathbb{1},
$$

where typically $\sigma^{\prime}$ would be the stress in the material due to the elasticity of the solid.

An important follower of Terzaghi was Maurice Biot (1905-1985). He developed a theory of linear poroelasticity which is used up to this day, see for instance [32]. 
In the line of Fillunger, Clifford Truesdell (1919-2000), the founder of the modern rational mechanics school, laid the foundation of the modern theory of mixtures on which the modern theory of porous media is build [25, pp. 218-221]. de Boer and Coussy made important contributions to the formation of a rational framework incorporating finite strain and thermodynamical effects [20, p. 70]. For the purposes of this thesis, however, the distinction between the more empirical and theoretical school is irrelevant, since in the quasi-static linear poroelastic limit, they agree. In this limit, acceleration is neglected, $\partial^{2} \boldsymbol{U} / \partial t^{2}=0$, and we neglect any buoyancy effects, $\rho=\rho_{\mathrm{f}}$. By combining equation (1.3), (1.4) and (1.5), one obtains the following balance of linear momentum

$$
\nabla \cdot \boldsymbol{\sigma}^{\prime}=-\frac{\eta}{k} \phi_{\mathrm{f}}\left(\boldsymbol{v}_{\mathrm{f}}-\boldsymbol{v}_{\mathrm{s}}\right)
$$

which shows how fluid flow through the solid causes a stress response in the solid material, and vice versa. The Terzaghi effective stress is of the form of a linear elastic solid, with the elastic constants specifying the response of the porous material at a (hypothetical) vanishing fluid pressure.

Interestingly, this limit of the linear poroelastic theory agrees with the gel dynamics theory for small deformations of a gel network [33], see section 1.2 for the definition of a gel. In this gel dynamics theory the force balance for a gel is derived through a form of the principle of virtual work. Under a variation of the deformation gradient in the hydrogel network, the resulting variation of the Helmholtz free energy of the network is required to vanish up to first order in the variation, that is, the free energy is minimized [33, pp. 3-6]. In this variation the fluid pressure enters as a Lagrange multiplier, needed to conserve the volume of the hydrogel, which stems from the (approximate) incompressibility of the fluid. The Terzaghi effective stress can be identified with the contribution of the hydrogel network to the free energy. This is reasonable, because the free energy of the network can be viewed as a surplus to the free energy of the fluid, similar to that the Terzaghi effective stress can be regarded as a surplus to the pressure that the penetrating fluid carries over to the network [27, pp. 5-6]. Next, assuming Darcy's law as a kind of linear response relation, the gel dynamics equations are obtained [33, p. 7]. Interestingly, the same set of equations can be obtained from a dissipative form of the principle of virtual work, called Onsager's variational principle [33-38]. In this approach one minimizes the so-called dissipation function which is a measure of the quantity of energy dissipated per unit of time for the dynamics of the gel. It has contributions from the friction 
between the viscous fluid flowing through the hydrogel network, and from the forces required to deform the network.

Concluding, for small deformations of the gel network the gel dynamics equations are of the exact same form as the equations of motion in quasi-static linear poroelastic theory. Therefore, we effectively treat the experimental systems of hydrogels (chapter 2), fibrin gels (chapter 3) and a model low fat mayonnaise (chapter 4) as porous media. In the next sections we will consider these experimental systems by delving into their background, relevance and applicability.

\subsection{Hydrogels}

In chapter 2 we treat the response of hydrogels on diffusing colloidal particles which can penetrate the hydrogel from the surroundings. Here, we introduce the varieties of hydrogels and the historical background of their application.

A hydrogel is understood to be a three-dimensional polymer network that can imbibe in its swollen equilibrium state a large amount of water, relative to its dry volume [39]. The hydrophilicity of hydrogels is due to the presence of chemical groups such as hydroxylic- $(-\mathrm{OH})$, carboxylic- $(-\mathrm{COOH})$, amidic- $(-\mathrm{CONH}-)$, primary amidic- $\left(-\mathrm{CONH}_{2}\right)$ and sulphonic $\left(-\mathrm{SO}_{3} \mathrm{H}\right)$ groups in the polymer backbone chain and/or the lateral chains [40]. There exists an enormous variety of hydrogels. They can be composed of natural or synthesized polymers, or a hybrid combination of the two. The polymers can be cross-linked chemically, that is, with covalent bonds, or they can be cross-linked physically, that is to say, with non-covalent bonds like hydrophobic and ionic interactions. Homopolymer hydrogels are composed of a single kind of monomer, whereas copolymer and multipolymer hydrogels are composed of, respectively, two or more kinds of monomer. Moreover, when swollen with water the monomers can be non-ionizable, ionizable and they may be ampholytic and zwitterionic. The pore size of a hydrogel determines the time scale on which it can change its volume by imbibing or expelling water. So-called non-porous hydrogels have a pore size of the same order of magnitude as the monomers of which the polymers are composed, namely, the pore size is typically $1-10 \mathrm{~nm}$. Additionally, microporous and macroporous hydrogels have pore sizes ranging from, respectively, 10-100 nm and $0.1-1 \mu \mathrm{m}$ [40]. The gels we study in chapter 2 and 3 can be classified, respectively, 
as microporous synthetic hydrogels and macroporous biopolymer hydrogels.

The term 'hydrogel' was already coined in, at least, the year 1894; by then it denoted a colloidal gel of inorganic salts $[39,41]$. The term 'gel' is even older, as sol-gel chemistry was already used by Thomas Graham (1805-1869), who is known as the father of physical chemistry, to make silica gels [42, p. 1]. This kind of gels is well known from, for example, the beads used in parcels to keep their contents dry during transportation. The study of the physical properties of polymeric gels resides mainly around the middle of the $20^{\text {th }}$ century with the work of Paul Flory (19101985), Maurice Huggins (1897-1981) and Leslie Treloar (1906-1985) [43-48]. The first industrial-size application of polymeric hydrogels was by Wichterle \& Lim in 1960 [49]. They searched for materials suitable for alloplastic and prosthetic use because plastic prosthesis can cause mechanical irritation and irritant substances may diffuse from the plastic into the surrounding tissue of the patient. Therefore, they aimed for a material which could somewhat mimic human tissue. It was required to have high water content, to be inert against biological processes and to be permeable for metabolites [49]. A main application they found for synthesized poly(2hydroxyethyl methacrylate) (pHEMA) hydrogels were contact lenses [50, p. 31]. Subsequently, research into hydrogel applications took off [51] and hydrogels are now present in the domestic sphere through, for example, disposable diapers [52], cosmetics [53, 54] and watering beads for plants. Also, hydrogels have found application in the medical sphere with applications like tissue engineering [55], bone regeneration [56], wound healing [57] and drug delivery [58]. Given these medical applications, one needs little imagination to see that the deformation of hydrogels in response to diffusing colloidal particles from their surroundings is an interesting and worthwhile topic of study. Some of the newest (prospective) fields of hydrogel application lie in metamaterials [59], agriculture [60-62] and wastewater treatment [63].

After Wichterle \& Lim, many new paths were openend in the field of hydrogel research. These paths can be described in terms of generations as follows, where we paraphrase a review by Buwalda et al. [39]. For two decades after Wichterle \& Lim's main discovery in 1960, the first generation of hydrogel research was aimed mainly at chemically cross-linked synthetic polymers of which pHEMA, poly(vinyl alcohol) (PVA) and poly(ethylene glycol) (PEG, also called PEO) are common examples. In the seventies a second generation of physically cross-linked hydrogels entered the 
stage which were responsive to their environmental conditions like the $\mathrm{pH}$ and the temperature. A third generation of hydrogels arose in the middle of the nineties comprising yet other crosslinking methods like stereocomplexation, inclusion-complex formation, model-ligand coordination and peptide interactions. The most recent generation is that of 'smart' hydrogels. An example of smart hydrogels are double network hydrogels where a combination of physical, covalent and ionic bonds is utilized such that their mechanical stability and release kinetics can be controlled. In a way, the model light mayonaise which we study as a model system in chapter 5 can be considered as a double network hydrogel for it is thought to feature both a network of oil droplets as well as an amylose network which originates from cooked starch.

As noted above, hydrogels were developed as biocompatible materials, that is, materials which are well compatible with tissues, for instance. This is closely related to the next section, where we discuss the historical background of research into the mechanical aspects of biopolymer networks and tissues.

\subsection{Biomechanics and fibrin fibers}

In chapter 3 we develop a new method to determine the poroelastic properties, that is, the hydraulic and elastic properties, of biopolymer networks and tissues. Considering biopolymer networks and tissues as gels, we develop a theoretical model which accounts for the stress and strain in the gel network, and the flow of fluid through the network. Experimentally, we investigate the compression of fibrin gels, which are model systems for blood clots. To place these results in a wide context, we give a brief account of the history of the field of biomechanics, and shortly touch on the related fields of biophysics and mechanobiology. Also, we give an introduction into the model system we used: fibrin gels.

Biomechanics can be said to be the science of 'mechanics applied to biology' [64]. It considers different components of organisms, from the molecular to the macroscopic scale, and aims to elucidate the mechanical aspects associated with them. A plethora of applications is derived from this science: the understanding of bone failure [65], plastic surgery [66], surgical robots [67], tissue engineering [68], etc. The history of biomechanics is, similar to physics, a long one $[69,70]$ and features, as with physics, Aristotle as one of its founders [71]. Through the ages we meet many famous 
names [72] like Leonardo da Vinci (1452-1519) with his treatise on locomotion, for instance. Modern biomechanics was established only in the second half of the $20^{\text {th }}$ century, however, with, among others, the introduction of mixture theory which allowed the investigation of poromechanical aspects of matter in living beings [69, p. 4].

A field closely related to biomechanics is biophysics, which comprises the study of biological subjects with approaches from the physical sciences [73]. The first generation of biophysicists may be identified with the so-called '1847 group': a group of four researchers that ".. imagined that we should constitute physiology on a chemicophysical foundation, and give it equal scientific rank with Physics..." [74]. The large growth of biophysics, however, similar to biomechanics, occurred in the second half of the $20^{\text {th }}$ century [75]. The physicalism dominating biophysics has been criticised as being an overly reductionist approach to biology in, for example, biosemiotics [76, pp. x,xi], but nevertheless biophysics has proven to be a fruitful field of research. The most recent program of inquiry in biophysics could be framed as the physics of modular cell biology. A module is considered to be a complex of interacting molecules in a cell having a well-defined function that is chemically isolated from other modules. Modules can be more or less connected to or insulated from other modules, depending on whether one function influences another and whether cross-talk of modules is harmful [77-79]. An example of a well-studied module is the cytoskeleton [8083].

A relatively new offspring from the field of biomechanics, in conjunction with the field of biophysics, is mechanobiology. It focuses on the interaction between mechanical strain and biological reaction. It does not stop with the passive mechanical properties of tissues, but studies the functional adaptation and tissue remodelling due to the interactions between cells, the interactions between cells and the extracellular matrix, and the mechanically induced biophysical changes in cells [69, 84-86]. The recent interest in mechanobiology might be related to the following historical considerations. It has been known for millennia that organisms are composed of fibrous connective tissue, and these fibers, e.g., collagen, were used in the production of a variety of commodities [87, p. 86]. Although the fibrous structure of tissues was well known, the development of cell theory, being a cornerstone of modern biology [88], was founded by Robert Hooke (1635-1703) in the $17^{\text {th }}$ century, and was completed in the $19^{\text {th }}$ century $[70, \mathrm{p} .14]$. With this scientific advance, tissues were recognized as 
being composed of cells and extracellular matrix, but the latter was initially viewed as an inanimate, nonreactive and purely structural component [87, p. 90]. This has proven to be an untenable view, however, and possibly partly explains the current interest in mechanobiology.

With this said, it is not too much of a surprise that the porous nature of the cytoskeleton and the extracellular matrix has received much scrutiny in recent years $[85,89-$ 92], next to the more classical work on the poromechanics of cartilage [93, pp. 245248]. In line with this earlier research, we expect our work on the compression of gels to provide a useful tool for both the determination of the poromechanical properties of soft biopolymer networks and tissues, and for the controlled study of the influence of inhomogeneous strain in tissues on cells in the extracellular matrix. Next, as we used fibrin gels as a model system to develop our theoretical model, we give a short introduction into the formation of fibrin fibers and their functioning, where we paraphrase the reviews of Weisel \& Litvinov [94] and Laurens et al. [95].

\subsubsection{Fibrin network formation}

In case of hemorrhage, i.e., the bleeding resulting from damage to a blood vessel, the process of hemostasis starts to stop the bleeding. The first step in this process is the formation of a 'plug' of blood platelets to seal off the wound. At the same time, a cascade of enzymatic reactions, resulting from the injury of the blood vessel, causes the conversion of a dissolved macromolecule in the blood - the protein called fibrinogen - into a network of fibrin fibers, in other words, a blood clot is formed. This blood clot further prevents bleeding and promotes healing of the wound. At the end of the enzymatic reaction cascade, an enzyme called thrombin appears. This enzyme catalyses the cleavage of fibrinopeptides from fibrinogen molecules, thereby creating fibrin monomers. These monomers form a half-staggered dimer and subsequent addition along the long axis of the monomers creates a double stranded protofibril containing 20-25 monomers, which has a length of $0.5-0.6 \mu \mathrm{m}$, see Figure 1.4 for an illustration. By aggregating along their short axis, the protofibrils form fibrin fibers; the mechanisms, structural motifs and driving forces of this aggregation are not well known. Due to branching of the fibrin fibers a space-filling fibrin fiber network is formed. Finally, the fibrin network is covalently cross-linked by the plasma transglutaminase Factor XIIIa. This enzyme catalyzes the covalent cross-linking of 


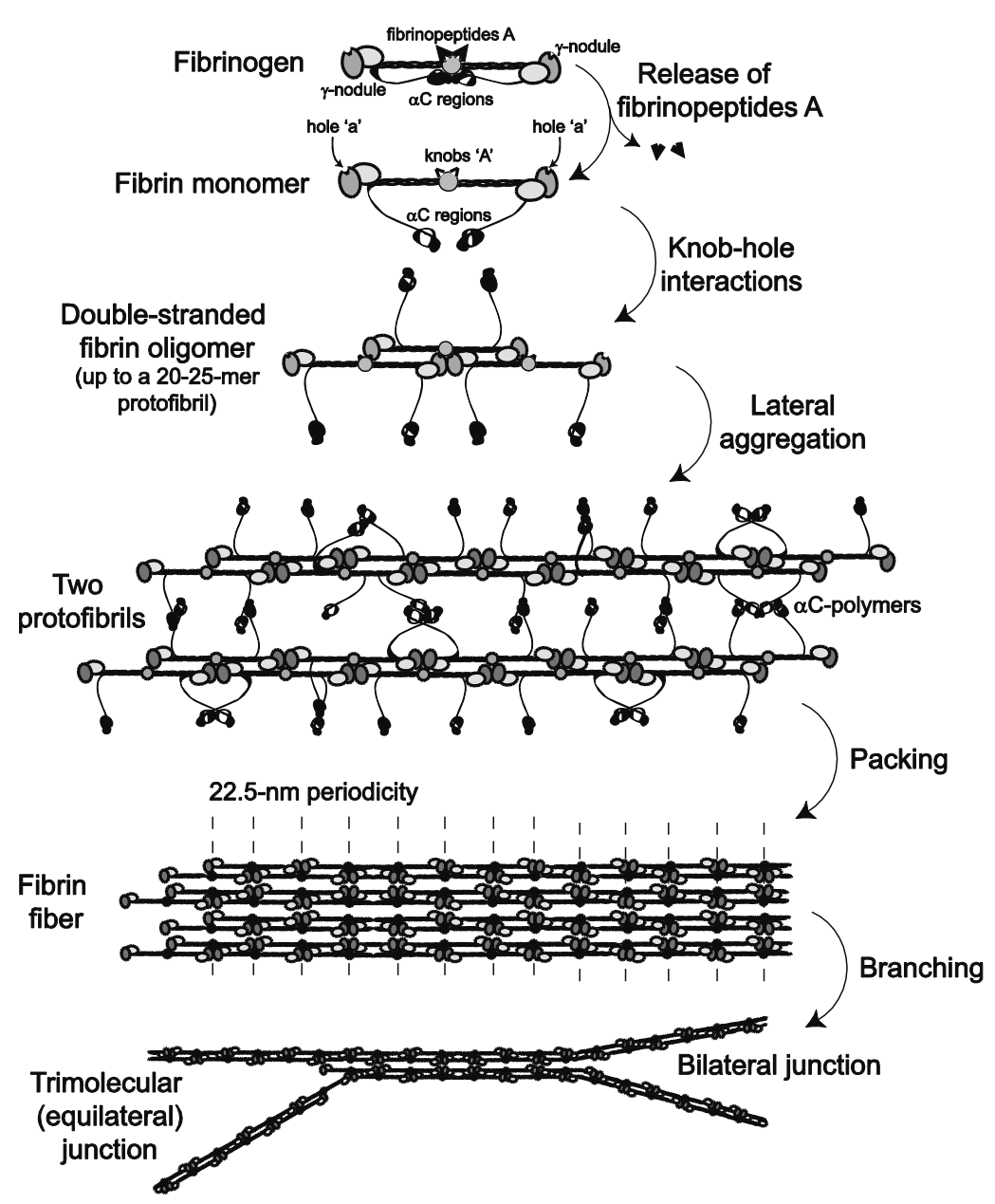

Figure 1.4: The formation of a fibrin fiber from fibrinogen macromolecules. After the enzyme thrombin cleaves fibrinopeptides from dissolved fibrinogen molecules, the resulting fibrin monomers polymerize spontaneously into a double-stranded fibrin oligomer. When the oligomer contains 20-25 monomers, it acts as a protofibril which can laterally aggregate with other protofibrils into a fibrin fiber. This fibrin fiber can branch and connect to other fibers to form a fibrin network: the structural component of a fibrin gel. The image is taken from Weisel \& Litvinov [94].

the fibrin fibers, thereby making the network mechanically stable.

Fibrin polymer networks posses a variety of mechanical properties, which is perhaps not unexpected given the complex structure of a fibrin network. From the viewpoint of material science a fibrin gel can be considered as a viscoelastic material: the fibrin network produces an elastic response and the interpenetrating fluid produces a 
viscous response. In this thesis we consider a fibrin gel as a biphasic material composed of a linear elastic solid, the fibrin network, and a viscous fluid. To consider the fibrin network as a linear elastic solid is tantamount to assuming an effective description of the network, because fibrin networks are known to exhibit strong nonlinear responses [96-99]. Fibrin networks strain stiffen when stressed [100] and they exhibit mechanical responses on different scales [94]. At the macroscopic scale $(\mathrm{cm})$ they can display dramatic decreases in volume when they are stretched [101]. On the mesoscopic level $(10 \mu \mathrm{m})$, fibers align in the direction where the network is strained and form bundles, thereby dramatically increasing the local elasticity [96]. Finally, on the microscopic $(\mu \mathrm{m})$ scale fibers buckle and bend in the direction of compression.

Practically, fibrin is used as a biomaterial in the medical realm. For example, it is used as a sealant to achieve hemostatis and tissue sealing in surgery [102, 103], and it is used to promote wound healing [104-106].

\subsection{Porous matter in industry}

As we have seen in section 1.1.2, a traditional subject in poromechanics is soil mechanics. This subject is still of importance for building physics and hydrology [107109]. Another classical application of porous medium theory is the retrieval of oil and gas from porous rock [110]. Relatively new fields of application are topics like bioengineering [111, 112], for instance, see section 1.3 for more information, and the industry of processed food [113]. The list of standard problems which may be encountered in these fields of application is a long one [114], but nevertheless we hope to add to this list with the approximate solution of chapter 3 .

The work described in this thesis was performed in the context of an industrial partnership program (IPP) between The Netherlands Organisation for Scientific Research (NWO) and Unilever R\&D Vlaardingen. The IPP is called 'Hybrid Soft Materials: From Physical Mechanisms to Designer Products', and its goal is to

"...understand and characterize the physico-chemical mechanisms that determine the structure and rheological properties of hybrid bio-inspired soft matter networks, with the ultimate goal of providing design principles for real consumer product applications" [115], 
a goal which may remind one of the goal of the pioneers in the field of biophysics that we encountered in section 1.3. One may ask, what is the overarching raison d'être of this IPP? In general, companies fabricate products which generate a profit when sold. For these products, the most important aspect, as seen from a company's perspective, is that they serve their purpose somewhere within a favourable spot in the parameter space spanned by, for example, product safety, production cost, consumer experience, shelf life, and product quality. To foster more control over these parameters, the IPP focuses on the rational understanding of food products, or food product related model systems. Apart from this, the interaction between science and industry may be profitable.

Ever since the production of mayonnaise became industrialized, and since the fat content of mayonnaise has become (considered to be) a problem, the replacement of the fat content with water required a natural additive which would thicken the mayonnaise [116]. An associated problem with using starch as an additive is the syneresis of low fat mayonnaise: the spontaneous excretion of fluid. In chapter 5 we study the physical mechanisms determining this fluid expulsion.

\subsection{Thesis outline}

Given the foregoing sketches of the historical background of the theory of poromechanics and the physical systems we study in this thesis, we outline the following research chapters.

In Chapter 2, we focus on the swelling and compression of hydrogel particles in polymer solutions. Experimentally, the hydrogel particles are found to exhibit non-monotonic (de)swelling under influence of the diffusion of polymer molecules into the hydrogel network during (de)swelling. Because the diffusion of polymer molecules decreases the osmotic pressure difference between the inside and the outside of the hydrogel particle, and because they interact with the hydrogel network, a rich phenomenology of (de)swelling occurs. To understand these processes, we develop two models, the first is a semi-phenomenological approach and the second is based on the theory of poroelasticity. The first applies relaxational model A dynamics [117], where we demand the free energy of the hydrogel-polymer system to descend to its minimum, and we numerically solve the resulting nonlinear equations of 
motion for the hydrogel radius as a function of time. In the second approach, we start by considering the dynamics of the diffusing polymer molecules and the resulting deformation of the hydrogel network in the limit of a dilute polymer solution. We solve the poroelastic equations of motion exactly using mode-expansions. Next, by making a dominant mode assumption, we formulate a closed-form model for hydrogelpolymer dynamics, allowing direct quantitative insight in the (de)swelling dynamics. With this model, the volume change of a spherical hydrogel, when swelling or shrinking in a polymer solution, can be calculated straightforwardly. By fitting the calculated volume change to measurement, both models allow to estimate the hydrogel network bulk modulus, its permeability, the diffusion constant of the polymer molecules, and the strength of the interaction between the polymer molecules and the hydrogel network.

In Chapter 3, we develop a novel method to measure the poromechanical properties of biopolymer networks, tissues, or any gel that can be clamped to the plates of a (commercial) parallel plate rheometer. We formulate an approximate closed-form solution for the displacement field of the solid network and the accompanying flow of fluid when a disk-like gel is ramp compressed. The solution is formed in analogy with the exact solution of a compressed gel experiencing no surface friction with the rheometer. To test this solution we compare the theoretically calculated normal force with the experimentally measured force from compression experiments on fibrin gels. From this comparison we find close agreement with the calculated time-dependent normal force for large-pore and small-pore fibrin gels, for gels having a wide range of fibrinogen concentrations, and for various compression rates. Moreover, we obtain estimations for the permeability and the (effective) elasticity of the fibrin networks. Interestingly, we find the effective elastic properties of fibrin networks to depend on the compression rate. By extending our model phenomenologically to incorporate strain stiffening, we find the onset stress, around which stiffening occurs, to depend on the fibrinogen concentration in a power-law fashion. Similarly, the permeability of small-pore fibrin networks is found to scale in a power-law fashion, a result which may provide input for research into the complex polymerization process of fibrin fibers.

In the first two research chapters we focus on the understanding and characterization of fluid filled (bio)polymer networks, namely, gels. In particular, the second chapter focused on compression tests. Compression tests are generally well understood, 
but problems with the misalignment of plates and shape-limited materials are known. Aiming to contribute to the solution of these problems, we consider in Chapter $4 \mathrm{a}$ compression test device with a novel plate-sphere geometry. In solving the corresponding boundary value problem, we present a novel method to solve the NavierCauchy equations of motion, i.e, the force balance for a linear elastic solid. By expanding the displacement field around the incompressible limit, we obtain a linear hierarchy of coupled equations. The applicability of this method is shown by solving for the displacement field of a cylindrical sample of material that is compressed with a spherically tipped plate. This compression-test geometry may have the advantage of reducing error in the determination of the Young's modulus of a material from compression measurements, and it may be useful to facilitate the pinning of lubricated shape-limited materials.

In the final research chapter of this thesis, Chapter 5, we focus on a model system for low fat mayonnaise exhibiting the spontaneous expulsion of fluid, that is, syneresis. Experimentally, this system is investigated in a conventional jar-tube setup which simulates a consumer jar of mayonnaise from which a scoop of mayonnaise is taken, thereby inducing fluid outflow due to hydrostatic pressure differences. Theoretically, we investigate the appropriateness of this setup by assuming the hypothesis that the membrane in the setup becomes clogged with the oil droplets present in the low fat mayonnaise. After ruling out this hypothesis, we propose that a double network in the mayonnaise, combined with fluid flow through the porous network, possibly explains the observed expulsion of fluid.

Finally, we end this thesis by treating the foregoing results in a general discussion in which we discuss our findings and relate them to the wider scientific context. Also, we propose some opportunities for future research. 


\section{Chapter 2}

\section{The swelling and compression of hydrogels in concentrated polymer solutions}

The results of this chapter are contained in ${ }^{1}$ :

M.T.J.J.M. Punter, P. van der Schoot, H.M. Wyss \& B.M. Mulder. "The compression and swelling of hydrogels in polymer solutions: a dominant-mode model". In: preparation.

F.J. Aangenendt*, M.T.J.J.M. Punter*, B.M. Mulder, P. van der Schoot \& H.M. Wyss. "Non-monotonic swelling and compression dynamics of hydrogels in polymer solution". In: submitted (2019).

* These authors contributed equally to this work.

\footnotetext{
${ }^{1}$ The experiments reviewed in this chapter have been designed and carried out by Jelle J.F. Sleeboom, Frank J. Aangenendt, and Hans M. Wyss from the Technical University of Eindhoven (The Netherlands).
} 


\section{Abstract}

The swelling and compression of hydrogels in polymer solutions can be understood by considering hydrogel-osmolyte-solvent interactions which determine the osmotic pressure difference between the inside and the outside of a hydrogel particle and the changes in solvent quality for the hydrogel network. We incorporate both these effects in a semi-phenomenological relaxational dynamics model as well as in a dominant-mode model based on the theory of poroelasticity. Specifying the free energy of a hydrogel particle, the relaxational dynamics model predicts the evolution of the volume of a hydrogel particle as a function of time. Using the theory of poroelasticity, we find the exact solution to hydrogel dynamics in a dilute polymer solution, which quantifies the effect of diffusion and partitioning of osmolyte, and the related solvent quality change, on the volumetric changes of the hydrogel network. By making a dominant mode assumption, we propose the dominant-mode model for the swelling and compression dynamics of spherical hydrogels in concentrated polymer solutions. Osmolyte diffusion induces a bi-exponential response in the size of the hydrogel radius, whereas osmolyte partitioning and solvent quality effects induce mono-exponential responses. Comparison of both the relaxational dynamics model and the dominant-mode model to experiments, provides reasonable values for the bulk modulus of a hydrogel particle, the permeability of the hydrogel network and the diffusion constant of osmolyte molecules inside the hydrogel network. Our model shows that the dynamics of hydrogel-osmolyte interactions can be conceptually simple and we expect our approach to provide a roadmap for further research into hydrogel dynamics and applications of hydrogel dynamics induced by, for example, changes in the temperature and the $\mathrm{pH}$. 


\subsection{Introduction}

Hydrogels are hydrophilic crosslinked polymer networks which, when fully swollen, typically imbibe large volumes of water relative to their dry volume. They can have strong volumetric responses to changes in, for example, the $\mathrm{pH}$, the temperature, and the osmotic pressure of their surroundings, which makes them interesting for drug delivery and responsive materials like actuators and soft robotics [39, 118, 119].

Recently, the compression and reswelling of microgel particles in response to an osmotic shock has been reported [120]. Initially fully swollen microgels were brought into a continuously flowing polymer solution and from both phenomenological and formative poroelastic modelling of their volumetric response, the diffusion constant of osmolyte inside the hydrogels, the permeability and the bulk modulus of the hydrogel network could be inferred. Due to nonlinearities, both the phenomenological and poroelastic model were solved numerically.

Here, we report experiments with remarkable overshoot behaviour of hydrogels swelling in polymer solutions. First, we describe these experiments using a numerically solved relaxational dynamics model based on the free energy of a hydrogel particle, which extends the previous phenomenological model [120]. This model provides little explicit insight in the resulting underlying hydrogel dynamics, however. In particular, the question remains what explicit quantities determine whether an overshoot or undershoot in the hydrogel volume occurs before it relaxes to equilibrium.

To remedy this deficiency, we also propose, using the theory of poromechanics, a dominant-mode model describing the volumetric response of hydrogels for both swelling and compression in concentrated polymer solutions. Large volumetric changes of hydrogels typically give rise to nonlinearities in the bulk modulus of the hydrogel, the permeability of the hydrogel network and the diffusion coefficient of the osmolyte. To obtain explicit insight in the hydrogel dynamics, we solve the osmolyte mass conservation equation and the force balance equation exactly for hydrogels in dilute polymer solutions. We find the temporal effect of osmolyte diffusion on the hydrogel radius to be of bi-exponential form, expressing the interplay between osmolyte that stresses the hydrogel network and the resulting 'diffusion' of the hydrogel network $[33,121]$. Partitioning of the osmolyte between the hydrogel and the 
surrounding polymer solution, and changes in the quality of the solvent for the hydrogel network induce mono-exponential swelling or compression to the equilibrium state, determined solely by hydrogel network diffusion. From this exact solution we approximate the hydrogel response to concentrated polymer solutions by making a dominant mode assumption.

Both the extended relaxational dynamics model and the dominant-mode model are fitted to the measured volumetric response of hydrogels from both Sleeboom et al. [120] and additional experiments. Reasonable values are obtained for the (effective) diffusion constant, permeability and bulk modulus, which scale as expected with the concentration of hydrogel network and osmolyte.

After briefly introducing the swelling and compression experiments on hydrogel particles we (re)analyze here, we first introduce the phenomenological relaxational dynamics model and compare it to experiments. Next, the equations of motion of hydrogel dynamics are formulated using the theory of poroelasticity. With the exact solution of hydrogel-osmolyte dynamics in a dilute polymer solution, we formulate the dominant-mode model. Finally, the dominant-mode model is compared to the experiments and the estimations for the bulk modulus, the hydrogel permeability and the osmolyte diffusion constant are discussed.

\subsection{Swelling and compression experiments}

\subsubsection{Microgels}

In the experiments of Sleeboom et al. [120] microgels with various acrylamide monomer concentrations and cross-linker densities were synthesized. This resulted in 'soft', 'medium' and 'stiff' microgel particles, depending on their estimated bulk modulus, as assessed with Capillary Micromechanics [122]. The microgels were trapped in a microfluidic device where they were first equilibrated in demi-water and subsequently compressed by replacing the demi-water with a continuously flowing polymer solution of dextran 70k (from Leuconostoc spp, $M_{w}=70 \mathrm{kDa}$, SigmaAldrich, radius of gyration $R_{\mathrm{g}} \approx 6 \mathrm{~nm}$ [123]), see Figure 2.1A and 2.1B. The hydrogels initially decrease in volume and either reswell to (approximately) their original volume or to some reduced volume, see Figure 2.1C-F. Each of the Figures 


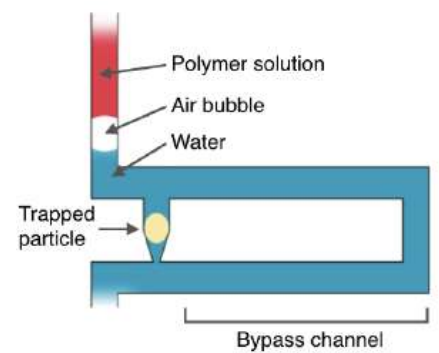

(A)

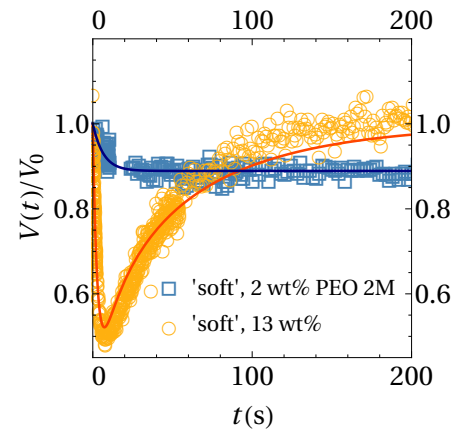

(D)

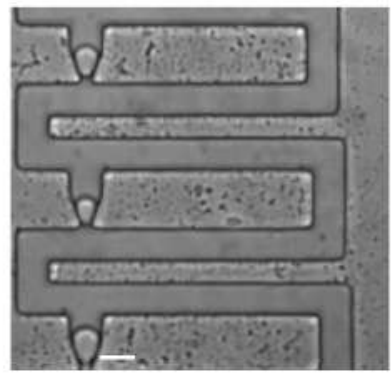

(B)

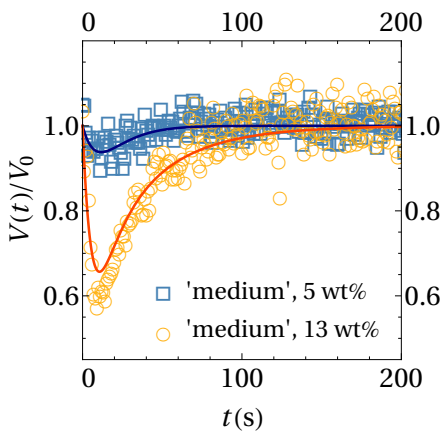

(E)

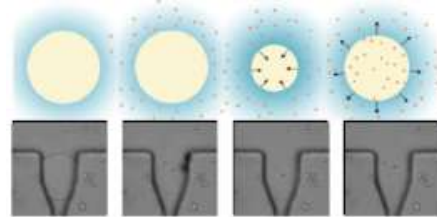

(C)

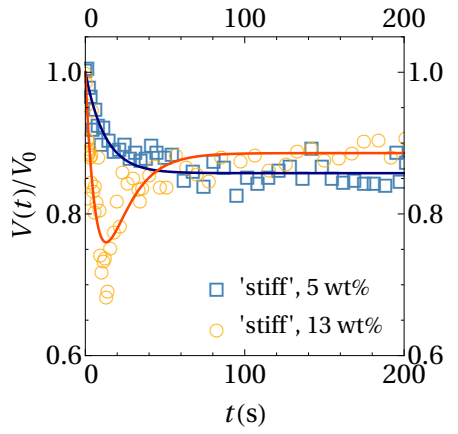

(F)

Figure 2.1: Schematic (A) and microscopy (B) image of the microfluidic setup in which microgels, with radius $a=20 \mu \mathrm{m}$, are trapped and the surrounding water (blue) can be replaced by a polymer solution (red). The ratio of the volume $V(t)$ of 'soft' (D), 'medium' (E), and 'stiff' (F) microgels to their original volume $V_{0}$ is given as a function of time $t$ after water is replaced by a dextran 70k polymer solution, unless specified otherwise. Replacing water by a PEO 2M polymer solution (D) shows that a large osmolyte causes permanent compression of the hydrogel particle. The solid lines are fits to the relaxational dynamics model, with the fitted parameters displayed in Table 2.1.

2.1D-F show that a higher osmotic pressure induces a faster initial decrease in hydrogel volume, but, see Figure $2.1 \mathrm{~F}$, not necessarily a smaller equilibrium volume. Therefore, apart from the osmotic pressure, solvent-quality effects are expected to be relevant for the determination of the equilibrium volume. Also, if PEO 2M is taken as an osmolyte molecule, see Figure 2.1D, no reswelling occurs, showing that the occurrence of reswelling is related to the size of the molecules. 


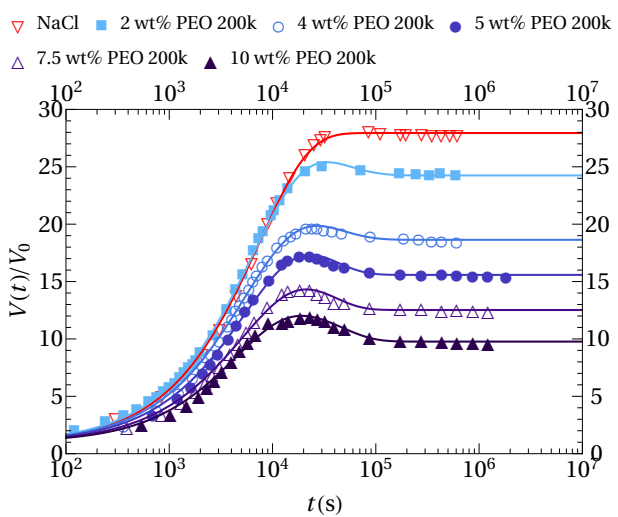

(A)

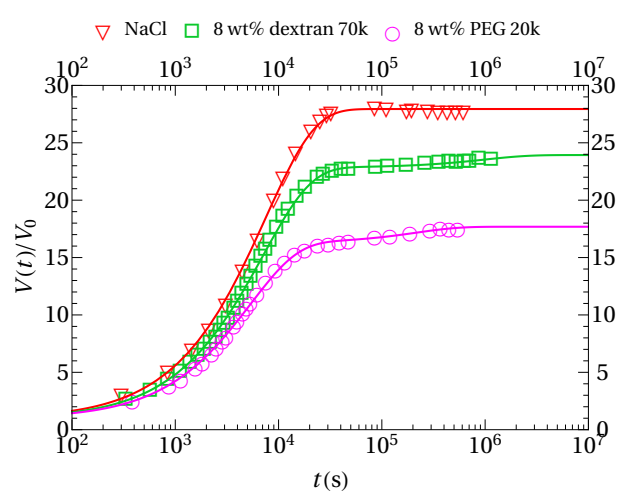

(B)

Figure 2.2: The normalized volume $V(t) / V_{0}$ as a function of time $t$ of initially dry Aquapearl hydrogel particles for different PEO 200k concentrations exhibiting an overshoot (A), and for dextran 70k and PEG 20k exhibiting no overshoot (B). The solid lines are fits of the relaxational dynamics model, with the fit and material properties displayed in the upper half of, respectively, Table 2.2 and Table 2.3.

\subsubsection{Aquapearls}

We use so-called Aquapearls (sodium poly-acrylate particles, Deco-Boulevard, Lohmar, Germany) as model hydrogel particles of macroscopic size. Swelling experiments were conducted by having dried hydrogel particles of average initial radius $R_{\text {dry }}=1.21 \mathrm{~mm}$ swell in polymer solutions of dextran $70 \mathrm{k}$, polyethylene glycol $20 \mathrm{k}$ (PEG 20k, $M_{w}=20 \mathrm{kDa}$, Sigma-Aldrich, $R_{\mathrm{g}} \approx 7 \mathrm{~nm}$ [124]) and polyethylene oxide 200k (PEO 200k, $M_{w}=200 \mathrm{kDa}$, Sigma-Aldrich, $R_{\mathrm{g}} \approx 22 \mathrm{~nm}$ [124]) at various osmolyte concentrations, see Figure 2.2. With increasing PEO 200k concentration, the equilibrium radius decreases, while the overshoot occurs at approximately the same time. For $t \gtrsim 10^{5} \mathrm{~s}$, there seems to be additional slow deswelling of the hydrogel particles, see in particular the $10 \mathrm{wt} \%$ PEO 200k experiment in Figure 2.2A. The PEG 20k and dextran 70k swelling experiments, on the other hand, exhibit slow swelling for $t \gtrsim 10^{5} \mathrm{~s}$, suggesting that the kind of osmolyte molecule determines the slow (de)swelling. Compression experiments show, similar to the swelling experiments, slow (de)swelling, see Figure 2.3. The PEO 200k compression experiments exhibit slow deswelling, and the PEG 20k and dextran 70k experiment show slow swelling, therefore the latter exhibits an undershoot in hydrogel volume. 


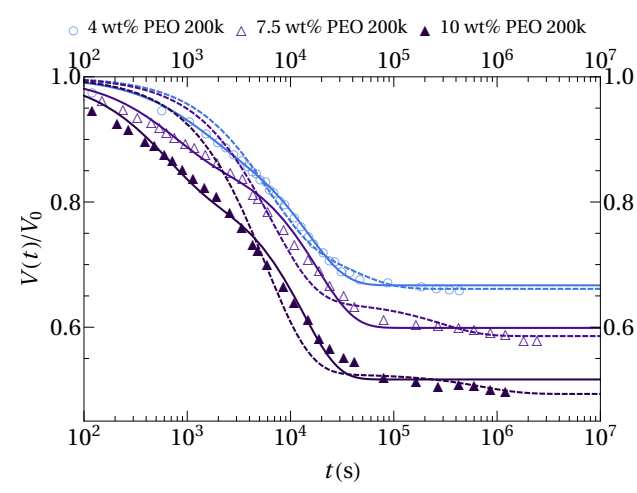

(A)

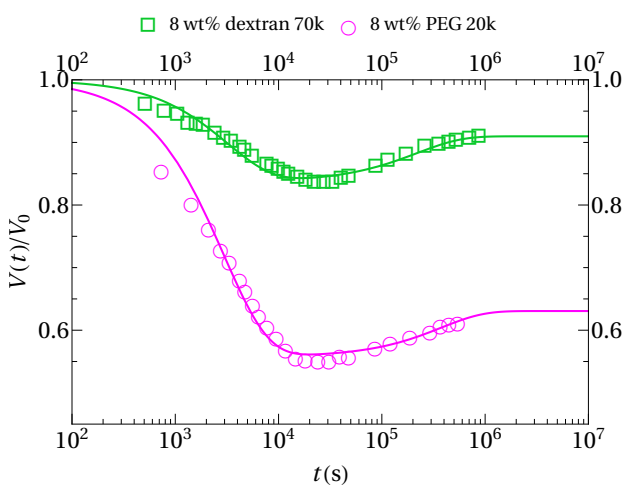

(B)

Figure 2.3: The normalized volume $V(t) / V_{0}$ as a function of time $t$ of Aquapearl hydrogel particles in different PEO 200k concentrations, exhibiting no undershoot (A), and for dextran 70k and PEG 20k exhibiting an undershoot (B). The solid lines are fits of the relaxational dynamics model, with the fit and material properties displayed in the lower half of, respectively, Table 2.2 and Table 2.3. The dashed lines in (A) are fits where the hydrogel (de)swelling time scale is fixed from the swelling experiments, thereby forcing the osmolyte diffusion to take into account the final stage of slow deswelling, see Table 2.4 for the fit parameters.

\subsection{Relaxational dynamics model}

We develop, based on the simple phenomenological model from our previous study [120], an extended relaxational dynamics model, which incorporates the modification of the solvent quality, brought about by the presence of macromolecules within the network of the hydrogel particles, as well as, related to this, a potentially uneven equilibrium distribution of these osmolyte macromolecules between the inside and the outside of the hydrogel, implying a remaining osmotic pressure difference.

We assume that if a particle is subjected to a pressure difference by immersing it in a polymer solution, this jump is instantaneous and that the pressure depends on the concentration of the osmolyte. We consider three separate regimes for the size of the osmolyte compared to the pores of the network:

The first regime is where the radius of gyration $R_{\mathrm{g}}$ of the osmolyte polymer chain is much larger than the network's mesh size $\zeta, R_{\mathrm{g}} \gg \zeta$. In this limit, we assume 
that the osmolyte does not penetrate the hydrogel network within experimental time scales.

The second regime is when the polymer chains are much smaller than the network's mesh size, $R_{\mathrm{g}} \ll \zeta$, in which case the diffusion of the molecules inside the network is nearly unhindered.

Describing the third regime between these two limiting cases, $R_{\mathrm{g}} \sim \zeta$, is not trivial. In our model we directly employ the diffusion coefficient of the osmolyte within the network $D_{\text {osm }}$ to describe the mobility of the polymer chains within the network. While this diffusion coefficient depends on the molecular weight of the polymer and the mesh size of the network, a theoretical prediction based on these parameters is not straightforward $[125,126]$. Due to the presence of the network, we assume that the diffusion of the osmolyte inside the particle is slower than diffusion of the osmolyte outside the particle. And because diffusion of osmolyte into the hydrogel is slow, the hydrogel particle responds elastically to the osmotic pressure difference caused by different concentrations of osmolyte inside and outside the gel particle. Therefore, we assume that initial volume changes are dominated by elastic effects.

At $t=0$ the osmolyte concentration inside the particle is 0 and the outside osmolyte concentration stays fixed during the experiment. Our simple model does not specifically take into account the spatial distribution of the osmolyte within the particle, instead regarding only the time dependent average osmolyte concentration within the entire particle. In our previous experiments [120], see Figure 2.1, we flow fresh background fluid past the particle, thereby ensuring that the outside concentration remains constant and homogeneous. To the same end, here we submerge the macroscopic hydrogel particles in a large container of background fluid under constant stirring of the fluid on a roller-bank, see section 2.6.1 of the Appendix. A large volume of the fluid reservoir ensures that any osmolyte absorption into the hydrogel does not significantly affect the outside concentration. As our background fluid has roughly 50 times more volume than the hydrogel particle this is a reasonable assumption. As we actively stir the solutions we also assume that the concentration of osmolyte outside the particle, and near the particle surface, is homogeneous.

Similar to our previous study [120], we assume model A dynamics [117] for the volumetric response of a hydrogel particle, which provides a governing equation for the 
radius of the particle $\dot{R}=-\Gamma \partial \Psi / \partial R$, where $\Gamma$ is a phenomenological rate constant and $\Psi$ is the free energy of the hydrogel. The free energy is taken to be

$$
\begin{aligned}
\Psi & =\overbrace{m \frac{3}{2} k_{\mathrm{B}} T\left(\frac{R}{R_{0}}\right)^{2}+m \frac{3}{2} k_{\mathrm{B}} T\left(\frac{R_{0}}{R}\right)^{2}}^{\text {elasticity }} \\
+ & \underbrace{N k_{\mathrm{B}} T(\log \rho v-1)}_{\text {mixing }}-\underbrace{N \mu}_{\text {uptake }}+\underbrace{\Pi \frac{4 \pi R^{3}}{3}}_{\text {expansion }}-\underbrace{k_{\mathrm{B}} T B m \frac{\rho}{\rho_{w}}}_{\text {interaction }},
\end{aligned}
$$

where the first two terms account for elastic deformations relative to the osmolytefree equilibrium state at radius $R_{0}$. The third term measures the mixing free energy of the osmolyte in the particle, the fourth quantifies the energetic cost of taking up osmolyte molecules from the surrounding solution, and the fifth measures the cost of hydrogel expansion in the solution. The last term, parametrized by $B$, is an interaction term with the role of a (cross) virial coefficient which can be positive or negative, depending on the interactions between the solvent, osmolyte and polymer network. We define $m$ as the number of cross-linked sub-chains, $N$ is the number of osmolyte molecules in the hydrogel particle, $\rho \equiv 3 N / 4 \pi R^{3}$ is the mean density of osmolyte inside the particle, $v$ is a microscopic volume scale, and $\mu$ and $\Pi$ are, respectively, the chemical potential and osmotic pressure of the osmolyte in the surrounding solution.

If the background fluid behaves like an ideal solution, we can apply van 't Hoff's law to the osmotic pressure $\Pi=k_{\mathrm{B}} T \rho_{w}$ of the outside solution, with $\rho_{w}$ the mean density of osmolyte. Due to hydrogel-osmolyte interactions the osmolyte concentration inside and outside the particle are not necessarily equal in the final equilibrium state, but are characterized by the equilibrium partition coefficient $Q$ [125], as

$$
Q \equiv \frac{3 N_{\mathrm{eq}} / 4 \pi R_{\mathrm{eq}}^{3}}{\rho_{w}},
$$

where $N_{\text {eq }}$ and $R_{\text {eq }}$ are, respectively, the number of osmolyte molecules inside the particle, and the particle radius in the final equilibrium state. The equilibrium partition coefficient measures the resulting ratio of osmolyte concentrations inside and outside of the hydrogel particle. In case the osmolyte molecules can be regarded as point particles, i.e., their radius of gyration $R_{\mathrm{g}}$ is much smaller than the mesh size 
of the hydrogel network $\zeta$, and if they experience no long-range interactions with the hydrogel, then $Q$ equals the volume fraction of solvent $\phi \equiv 1-\left(R_{\mathrm{dry}} / R_{\mathrm{eq}}\right)^{3}$, with $R_{\text {dry }}$ the radius of a dry hydrogel network. We estimate $Q$ as the fraction of available volume in the particle for a specific osmolyte, therefore all solvent-quality related interactions are absorbed in $B$. Thus, for osmolyte molecules of nonzero size able to penetrate the hydrogel particle, $0<Q<\phi$.

In principle, for a full description of the hydrogel-osmolyte-solvent interactions, we would require three Flory-Huggins-type $[44,45]$ parameters to quantify the impact of the solvent quality for both the network and osmolyte, and the interaction between network and osmolyte [127]. For simplicity, we choose a description using only the effective energetic parameter $B$.

Definition of the normalized particle radius as $\alpha(t) \equiv R(t) / R_{0}$ leads to a modified evolution equation for the particle radius

$$
\frac{\partial \alpha}{\partial t}=-3 \Gamma_{\alpha}\left[\alpha-\frac{1}{\alpha^{3}}-P\left(\frac{f Q \alpha_{\mathrm{eq}}^{3}}{\alpha}-\alpha^{2}\right)+B \frac{f Q \alpha_{\mathrm{eq}}^{3}}{\alpha^{4}}\right],
$$

where $P \equiv \Pi / K$ is the osmotic pressure scaled by the bulk modulus $K \approx$ $3 k_{\mathrm{B}} T m / 4 \pi R_{0}^{3}$ of the network, and $\Gamma_{\alpha} \equiv m \Gamma k_{\mathrm{B}} T / R_{0}^{2}$ is a relaxation rate. We defined $\alpha_{\text {eq }} \equiv R_{\text {eq }} / R_{0}$ as the ratio of the particle radius in the final equilibrium state $R_{\text {eq }}$ to that in the osmolyte-free state $R_{0}$, and we define $f(t)=N(t) / N_{\text {eq }}$ as the amount of osmolyte in the hydrogel particle at time $t$ relative to that in the final state, $N_{\text {eq. }}$.

To account for diffusion of the osmolyte from the fluid into the hydrogel particle, we use the diffusion equation in integral form, $\partial N / \partial t=D_{\text {osm }} \oint d^{2} S \cdot\left[\rho \nabla \mu / k_{\mathrm{B}} T\right]$ across the interface, where $D_{\text {osm }}$ is the diffusivity of the osmolyte within the gel. We treat the concentrations inside and outside the hydrogel particle as uniform but different. On dimensional grounds, this becomes $\partial N / \partial t=C D_{\mathrm{osm}} R^{-2}\left(N_{\text {eq }}-N\right)$ with $R(t)$ the radius of the hydrogel particle and $N_{\text {eq }}$ the number of osmolyte particles in the final equilibrium state; $C$ is a proportionality constant to be determined by comparison with the known solution of the diffusion equation for osmolyte molecules diffusing through a static hydrogel network, see Section 2.3.2. In normalized form, the diffusion equation reads

$$
\partial f / \partial t=-\Gamma_{f} \alpha^{-2}(f-1),
$$


with $\Gamma_{f} \equiv C D_{\text {osm }} / R_{0}^{2}$ the kinetic parameter that sets the time scale for the osmolyte molecules to enter the hydrogel particle by diffusion. The final equilibrium radius $R_{\text {eq }}$ can be found from equation (2.3) and (2.4) in the static limit.

If the osmolyte cannot penetrate into the hydrogel, $Q=0$ and the particle will simply compress or swell monotonically under influence of the applied constant pressure, see the right column in Figure 2.4. For $B=0$ the osmolyte does not alter the solvent quality for the hydrogel network, and the final volume is determined by the osmotic pressure difference between the inside and the outside of the particle, see the top row in Figure 2.4. By ignoring long range interactions and regarding the hydrogel network as a phantom network, i.e., $B=0$ and $Q=\phi \approx 1$, the model reduces to the version we described in Sleeboom et al. [120], see the top left scenario in Figure 2.4. In this case, the osmolyte can penetrate the network and does not interact with it in any way; as a consequence, in the final equilibrium state the concentrations inside and outside the hydrogel particle are the same.

For $B \neq 0$ and $Q>0$ the osmolyte penetrates the gel and the solvent quality is affected by the presence of the osmolyte. This generally results in a modification of the final volume, except in the special case where the osmotic pressure difference between the inside and the outside of the particle balances the effect of increased solvent quality, i.e., $B Q-P(Q-1)=0$. Using this relation, we reanalyze the experiments of Sleeboom et al. [120] for the 'soft' and 'medium' hydrogel particles, see Figure 2.1. For the experiments in the present study, the illustrations in the middle column at the middle row of Figure 2.4 summarize the different qualitative behaviors for both swelling and compression experiments. These illustrations show how a small decrease in solvent quality (dotted curve) can cause an undershoot in a compression experiment, whereas a large decrease in solvent quality (solid curve) can cause an overshoot in a swelling experiment.

\subsubsection{Fitting results}

By fitting the extended relaxational dynamics model to the experimental data we can obtain the permeability $k$, the bulk modulus $K$, the diffusivity of the osmolyte in the hydrogel $D_{\mathrm{osm}}$, and an indication for the change in solvent quality due to the osmolyte in the form of the parameter $B$. We fit our model to our experiments using the Mathematica functions NonlinearModelFit and NDSolve where we set $P, \Gamma_{\alpha}$, 


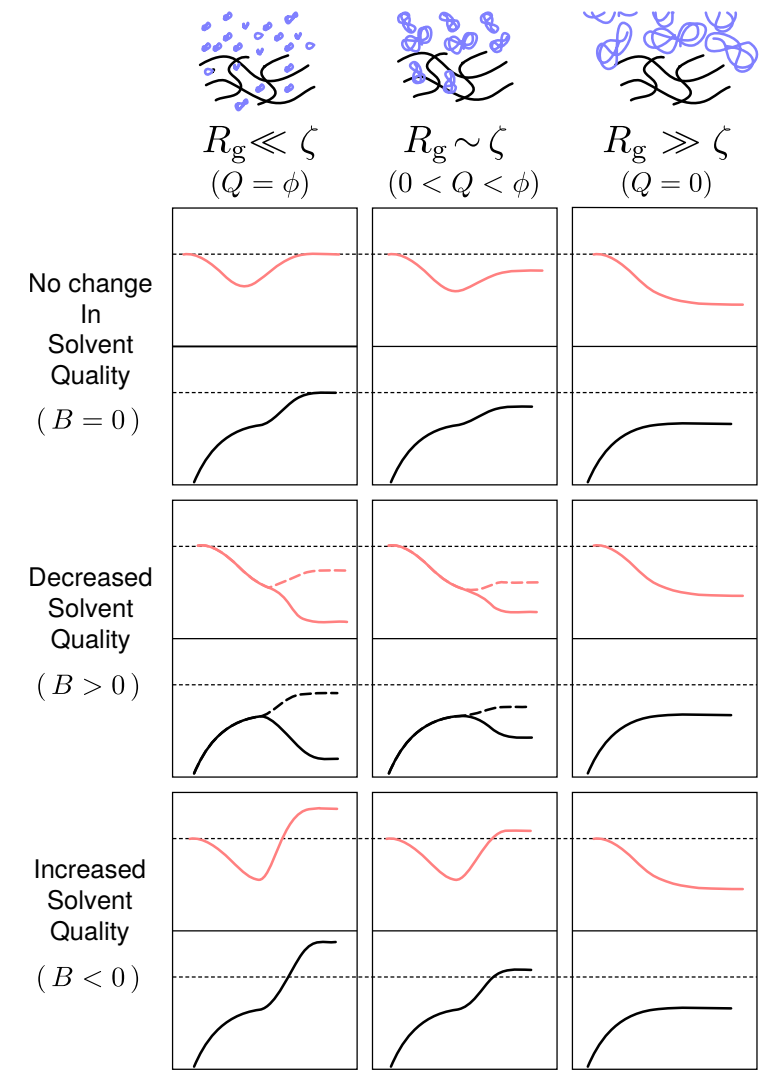

Figure 2.4: Schematic illustration of the different theoretical scenarios for the time evolution of the volume of a hydrogel particle on a log-linear scale under compression (solid red lines) and swelling (solid black lines) in polymer solutions, as a function of both the ratio of the radius of gyration $R_{\mathrm{g}}$ of the dissolved macromolecules to the mesh size of the hydrogel network $\zeta$ (columns) and their effect on the solvent quality of the solution $B$ with respect to the hydrogel network (rows). The horizontal dotted lines indicate the osmolytefree equilibrium level of swelling of the hydrogel particle. If $R_{\mathrm{g}} \ll \zeta$ or $R_{\mathrm{g}} \sim \zeta$, osmolyte can diffuse into the hydrogel particle (left and middle column). When there is no change in solvent quality (top row), the difference in osmotic pressure between the inside and the outside of the particle decreases over time due to osmolyte diffusion, causing slow (additional) swelling. A small decrease in solvent quality (middle row, dotted curve) can cause an undershoot in a compression experiment, whereas a large decrease in solvent quality (middle row, solid curve) can cause an overshoot in a swelling experiment. Increases in solvent quality (bottom row) enhance the magnitude of slow (re)swelling.

$\Gamma_{\mathrm{s}}$ and $B$ as fitting parameters, and we estimate $Q$ as follows.

The equilibrium partition coefficient $Q$ is defined as the ratio of the concentration of 
osmolyte molecules inside the hydrogel particle to the concentration outside of the particle in equilibrium, see equation (2.2). Both short-range and long-range interactions determine the equilibrium partitioning of osmolyte. Short-range interactions between the osmolyte and the hydrogel network set the fraction of available volume by determining the excluded volume through hard-core repulsive interactions. Long-range interactions, on the other hand, set the chemical potential for osmolyte molecules in the available volume inside the hydrogel particle. In our model, we account for long-range interactions in the energetic parameter $B$. Therefore, we estimate $Q$ as the fraction of available volume $\phi_{\text {av }}$ in the hydrogel particle, based purely on short-range interactions. Due to the finite size of osmolyte molecules, the fraction of available volume does not generally equal the equilibrium volume fraction of solvent $\phi \equiv 1-\left(R_{\mathrm{dry}} / R_{\mathrm{eq}}\right)^{3}$, with $R_{\mathrm{dry}}$ the radius of a fully dried hydrogel particle and $R_{\text {eq }}$ its radius in equilibrium.

To estimate the available equilibrium volume fraction in the Aquapearl hydrogels for 70k dextran, 20k PEG and 200k PEO, we model the available volume of a hydrogel particle as being comprised of spherical solvent-filled impenetrable shells with an inner radius equal to the mesh size $\zeta$ of the hydrogel network. Assuming the osmolyte particles to be hard spheres whose radius equals their radius of gyration $R_{\mathrm{g}}$, the available volume fraction for an osmolyte molecule moving in a shell equals

$$
\phi_{\mathrm{av}}=\left(1-R_{\mathrm{g}} / \zeta\right)^{3} .
$$

To estimate $\phi_{\mathrm{av}}$ for dextran 70k, PEG 20k and PEO 200k in the Aquapearl particles, we first estimate the available volume fraction for dextran 70k using equation (2.5). To do so, we calculate the ratio of the mesh size of the Aquapearl particles $\zeta_{\text {aqua }}$ to the mesh size of a $5 \mathrm{wt} \%$ poly-acrylamide network $\zeta_{\text {poly }}$ with known $\phi_{\mathrm{av}}$. We find the ratio of the mesh sizes by comparing the permeabilities $k \propto \zeta^{2}$ of the two kinds of hydrogel particles.

As a proxy for the available volume fraction $\phi_{\mathrm{av}}$ of the $5 \mathrm{wt} \%$ poly-acrylamide network for dextran $70 \mathrm{k}$ ( $R_{\mathrm{g}}=6 \mathrm{~nm}$ [123]), we use the measured available volume fraction of a $6 \mathrm{wt} \%$ polacrylamide hydrogel for dextran molecules with a radius of $6.42 \mathrm{~nm}$ [126]. As a proxy for the permeability of the Aquapearl particles, we use the result of section 2.6.5 in the Appendix, where we find the permeability of the Aquapearl particles to be $k=8(5) \mathrm{nm}^{2}$. To determine the permeability of $5 \mathrm{wt} \%$ poly-acrylamide hydrogels, we fit our model to the compressive response of $5 \mathrm{wt} \%$ 
Table 2.1: Material properties of the soft, medium and stiff poly-acrylamide hydrogels of Sleeboom et al. [120] from fits with the relaxational dynamics model. For the bulk modulus of the medium and the stiff particles we used the value obtained from Capillary Micromechanics, see the Supplemental Material of Sleeboom et al., and for the soft particles we used the estimation of the bulk modulus from the fits in Sleeboom et al. The weight percentage indicates the concentration of the dextran 70k polymer solution used in the experiment. The standard error for each derived material parameter is given in brackets. We assumed all fitted parameters to be uncorrelated.

\begin{tabular}{lcccc} 
Experiment & $\begin{array}{c}\Pi \\
{[\mathrm{kPa}]}\end{array}$ & $\begin{array}{c}K \\
{[\mathrm{kPa}]}\end{array}$ & $\begin{array}{c}k \\
{\left[\mathrm{~nm}^{2}\right]}\end{array}$ & $\begin{array}{c}D_{\mathrm{osm}} \\
{\left[\mu \mathrm{m}^{2} / \mathrm{s}\right]}\end{array}$ \\
\hline \hline Medium 13wt\% & 29.4 & 13 & $0.17(0.02)$ & $1.01(0.16)$ \\
Medium 13wt\% & 29.4 & 13 & $0.19(0.02)$ & $0.74(0.11)$ \\
Medium 5wt\% & 4.2 & 13 & $0.18(0.03)$ & $3.43(0.94)$ \\
Soft 13wt\% & 29.4 & 10 & $0.40(0.01)$ & $0.51(0.02)$ \\
Soft PEG 2M & 1.8 & 10 & $0.51(0.03)$ & $0.00(0.00)$ \\
Stiff 5wt\% & 4.2 & 17 & $0.15(0.09)$ & $7.36(307.00)$ \\
Stiff 13 wt\% & 29.4 & 17 & $0.09(0.01)$ & $2.46(0.34)$ \\
\hline \hline
\end{tabular}

'medium' poly-acrylamide microgel particles with dextran 70k from our previous work [120], see Table 2.1 for the fitted parameters. In making the fits for the medium and soft particles we enforced the particle to reswell to its original size by demanding that $B Q-P(Q-1)=0$, and we put $Q=\phi_{\mathrm{av}}=0.11$ for the medium and soft gels and $Q=\phi_{\mathrm{av}}=0.02$ for the stiff gels [126]. We average the fitted values for the permeability $k$ of the medium hydrogels to obtain an estimate for the permeability of the $5 \mathrm{wt} \%$ poly-acrylamide network.

Assuming the proportionality constant in the scaling relation for the permeability $k \propto \zeta^{2}$ to be equal for the poly-acrylamide and the Aquapearl particles, we find the ratio of the mesh size of the Aquapearl particles to that of $5 \mathrm{wt} \%$ poly-acrylamide medium hydrogels as $\zeta_{\text {aqua }} / \zeta_{\text {polyac }} \approx 7$. Then, using equation (2.5), and that the available volume fraction for dextran $70 \mathrm{k}$ of the $5 \mathrm{wt} \%$ poly-acrylamide hydrogels is $\phi_{\mathrm{av}}=0.11$ [126], we find the available volume fraction within the Aquapearl particles for dextran 70k as $\phi_{\mathrm{av}}^{\mathrm{dex}}=0.78$, where we used $R_{\mathrm{g}}=6 \mathrm{~nm}$ [123].

As we know the ratio of the radius of PEG 20k and PEO 200k molecules to that of dextran 70k, we find the fraction of available volume in the Aquapearls for PEG 20k 
Table 2.2: Fitting parameters for the model curve fits displayed in Figure 2.2 and Figure 2.3. The upper and lower sections of the Table display the swelling and compression cases, respectively. The standard error for each fitted parameter is given in brackets.

\begin{tabular}{lcccc} 
& $\begin{array}{c}P \\
{\left[10^{-1}\right]}\end{array}$ & $\begin{array}{c}\Gamma_{\alpha} \\
{\left[10^{-5} / \mathrm{s}\right]}\end{array}$ & $\begin{array}{c}\Gamma_{f} \\
{\left[10^{-5} / \mathrm{s}\right]}\end{array}$ & $\begin{array}{c}B \\
{\left[10^{-1}\right]}\end{array}$ \\
\hline \hline Nolution & 0 & $0.94(0.01)$ & - & - \\
dextran 70k 8wt\% & $3.3(0.1)$ & $0.75(0.01)$ & $0.10(0.12)$ & $1.9(0.6)$ \\
PEG 20k 8wt\% & $12.7(0.3)$ & $0.62(0.01)$ & $0.39(0.16)$ & $5.6(0.3)$ \\
PEO 200k 2wt\% & $0.0(0.8)$ & $1.03(0.02)$ & $2.71(1.20)$ & $5.2(1.2)$ \\
PEO 200k 4wt\% & $4.0(1.5)$ & $0.88(0.02)$ & $2.69(1.30)$ & $10.3(1.7)$ \\
PEO 200k 5wt\% & $4.9(2.9)$ & $0.79(0.04)$ & $3.39(1.28)$ & $17.2(2.8)$ \\
PEO 200k 7.5wt\% & $8.9(5.1)$ & $0.65(0.04)$ & $3.40(1.41)$ & $23.9(3.9)$ \\
PEO 200k 10wt\% & $19.2(2.6)$ & $0.54(0.01)$ & $1.95(0.50)$ & $30.0(1.6)$ \\
PEO 200k 4wt\%+ & & & & \\
PEG 20k 8wt\% & $23.7(1.5)$ & $0.52(0.01)$ & $1.83(0.31)$ & $18.9(0.3)$ \\
\hline dextran 70k 8wt\% & $2.8(0.0)$ & $1.96(0.10)$ & $0.44(0.03)$ & $0.9(0.0)$ \\
PEG 20k 8wt\% & $14.3(0.5)$ & $1.17(0.08)$ & $0.22(0.35)$ & $5.3(2.1)$ \\
PEO 200k 4wt\% & $1.8(0.1)$ & $5.83(0.78)$ & $7.23(1.27)$ & $12.9(0.2)$ \\
PEO 200k 7.5wt\% & $2.3(0.2)$ & $9.94(1.45)$ & $5.84(1.12)$ & $16.8(0.3)$ \\
PEO 200k 10wt\% & $3.4(0.3)$ & $10.90(1.73)$ & $8.16(1.68)$ & $22.0(0.4)$ \\
\hline \hline
\end{tabular}

and PEO 200k as $\phi_{\mathrm{av}}^{\mathrm{PEG}}=0.75$ and $\phi_{\mathrm{av}}^{\mathrm{PEO}}=0.36$, where we used $R_{\mathrm{g}}=7 \mathrm{~nm}$ for PEG 20k [124], and $R_{\mathrm{g}}=22 \mathrm{~nm}$ for PEO 200k [124]. Finally, taking $Q=\phi_{\mathrm{av}}$, we fit the extended model to the volumetric measurements of the Aquapearl particles, see Figure 2.2 and Figure 2.3. The parameters obtained from this fitting procedure are summarized in Table 2.2.

We first analyze the parameters obtained for the hydrogels swollen in PEO 200k to verify that the parameters are consistent. For these hydrogels we observe an increase in $P$ for increasing polymer concentrations, which is as expected, as the external pressure, $P \propto \Pi$, increases with increasing polymer concentration. We expect that the decay rate for the particle radius $\Gamma_{\alpha}$ decreases with increasing polymer concentration, because a higher network polymer density in the particle lowers the permeability for solvent molecules, and indeed, we do observe a slight decrease in $\Gamma_{\alpha}$ for increasing polymer concentration. We also observe a trend for an increase of $\Gamma_{f}$ with 
increasing PEO 200k concentration, which is somewhat expected, as the mutual diffusion coefficient increases with polymer concentration [128]. Further, we observe an increase of the parameter $B$ with increasing PEO 200k concentrations. This is reasonable, as the interaction term in equation (2.3) is not proportional to the absolute concentration of osmolyte molecules, and therefore the effect of increasing polymer concentration shows itself in $B$. The $P$ values for the hydrogels swollen in PEG 20k $8 \mathrm{wt} \%$ and dextran 70k $8 \mathrm{wt} \%$ are also consistent, as the PEG 20k solution has a much higher osmotic pressure than the dextran 70k solution, 65(9) $\mathrm{kPa}$ [129] versus $10 \mathrm{kPa}$ [123], respectively, where the estimated uncertainty, if available, is displayed in brackets.

For the compression experiments all the obtained values for dextran 70k and PEG 20k are comparable to those from the swelling experiments. For PEO 200k the obtained values for $P$ and $B$ in the compression experiments exhibit the same trends as those observed in the swelling experiments.

We find significant differences, however, between the values of $\Gamma_{\alpha}$ and $\Gamma_{f}$ obtained from the compression and the swelling experiments, respectively. While the rate of change of the particle radius to its equilibrium value $R_{\text {eq }}$ is set by $\Gamma_{\alpha}$, the rate constant $\Gamma_{f}$ sets the time scale for osmolyte diffusion, which may induce slow (de)swelling of the hydrogel particle by changing both the solvent quality and the osmotic pressure difference, see Figure 2.4. Therefore, if the measured volume does experience an under- or overshoot, as observed for the dextran 70k and PEG 20k compression experiments in Figure 2.3B the undershoot can be taken into account uniquely by the slow diffusion of osmolyte, thereby determining $\Gamma_{f}$. However, the PEO 200k compression experiments in Figure 2.3A exhibit no undershoot, thus making the effects contained in $\Gamma_{\alpha}$ and $\Gamma_{f}$ less clearly separated and their values prone to error.

\subsubsection{Material properties}

We can relate the model parameters to the bulk modulus $K$, the permeability $k$ and the diffusion coefficient of the osmolyte in the network $D_{\text {osm }}$. We determine $K$ directly from the fitted $P$ value as we defined $P=\Pi / K$. From literature we know $\Pi$ as a function of concentration, for both the dextran 70k [123] and the PEG 20k [129] solutions used in our experiments, but not for our PEO 200k solutions. To determine $\Pi$ as a function of concentration for our PEO 200k solutions, we place $1 \mathrm{~mL}$ samples 
of a 2 wt\% PEO 200k solution in dialysis bags and submerge them in dextran solutions of various concentrations with known osmotic pressures. After having given the samples a week to equilibrate, we extract the PEO 200k concentration as a function of osmotic pressure, assuming that the osmotic pressure inside has equilibrated to the pressure outside; see section 2.6.3 of the Appendix for details.

To determine the permeability $k$, we consider the (hypothetical) case of the swelling of an initially compressed hydrogel without osmolyte and find from equation (2.3) an exponential long-time relaxation at a rate of $12 \Gamma_{\alpha}$. Assuming that this rate corresponds to the dominant relaxation rate in the long-time limit of the analytical swelling model of Tanaka and Fillmore [121], we obtain $12 \Gamma_{\alpha}=\pi^{2} K k / R_{0}^{2} \eta$, with $\eta$ the dynamic viscosity of water. This expression is an improvement on the one of our previous work [120], where we compared the short-time swelling response of our phenomenological model to the long-time response of the analytical swelling model of Tanaka and Fillmore.

Finally, we can estimate $D_{\text {osm }}$ from the rate constant $\Gamma_{f}$. To do so, we need to realize that in our phenomenological model we had absorbed the unknown prefactor $C$ in the definition of $\Gamma_{f}$. To determine $C$, let us consider the (hypothetical) case where the particle radius remains fixed, $\Gamma_{\alpha}=0$, but diffusion of osmolyte can still occur. In this case, the only relevant process is the diffusion of osmolyte into the network, and we can solve equation (2.4) for all times, which yields $\Gamma_{f}$ as an exponential relaxation rate. Setting this rate equal to the dominant relaxation rate in the longtime limit of the analytical solution of the diffusion equation in a sphere of radius $R_{0}$ [130], we obtain $C=\pi^{2}$. This determination of $C$ is an improvement to our previous work [120], where we had put $C=1$.

We have placed all of these results in Table 2.3. From the swelling experiments (upper section of the table) we obtain an average value of 35(9) $\mathrm{kPa}$ for $K$ and 4(2) $\mathrm{nm}^{2}$ for $k$. We obtained these averages by weighting each value with the inverse square of its standard error; the corresponding standard deviation is displayed in brackets. These parameters are in fair agreement with the values of $K=27(6)$ $\mathrm{kPa}$ and $k=8(5) \mathrm{nm}^{2}$, obtained in separate, conventional experiments. To obtain these experimental values for $K$ and $k$, we used a macroscopic version of the Capillary Micromechanics technique [122] and a custom-built permeability measurement setup, respectively. See sections 2.6.4 and 2.6.5 of the Appendix. 
We notice that the osmolyte diffusion coefficient $D_{\text {osm }}$ obtained from the swelling experiments for the dextran 70k and the PEG 20k solutions is consistently lower than for the PEO 200k solution. The reason for this could be that, while overall the PEO 200k has a higher molecular weight than PEG 20k and dextran 70k, the fraction of the polydisperse PEO 200k species that is small enough to penetrate the hydrogel could still diffuse faster than the dextran 70k or PEG 20k polymers, see section 2.6.2 of the Appendix. Indeed, in experiments employing a mixture of PEG 20k and PEO 200k we obtain a diffusion coefficient $D_{\text {osm }}$ that lies between those observed for PEO 200k and for PEG 20k.

The anomalously high values for $K, k$ and especially $D_{\text {osm }}$ obtained from the PEO 200k compression experiments are possibly caused by the monotonic decrease of hydrogel volume in these compression experiments, which makes the effects of osmolyte diffusion $\left(\Gamma_{f}\right)$ and the rate of solvent permeation of the hydrogel particle $\left(\Gamma_{\alpha}\right)$ difficult to distinguish, see the last paragraph of section 2.3.1 for more information. Indeed, if we fix $\Gamma_{\alpha}$ with the value fitted from the corresponding swelling experiment, we find values for $K, k$ as well as $D_{\text {osm }}$ that are closer to those of PEG 20k and dextran 70k, see Table 2.4. The corresponding fit curves are displayed in Figure 2.3 as dashed lines.

Overall, given the simplicity of our model, for the swelling experiments the model accounts for the experiments surprisingly well, even as we neglected any polydispersity effects. Nevertheless, it could be useful to develop an improved model that specifically accounts for this factor.

From the results of Table 2.3 we conclude that the decrease in solvent quality is stronger for PEO 200k than for both dextran 70k and PEG 20k. This is a key ingredient needed to explain the long-time hydrogel particle dynamics in every swelling and compression experiment we conducted. In the experiments (part of) the osmolyte slowly diffuses into the hydrogel, as witnessed by the (de)swelling and (de)compression processes happening on long time scales in Figure 2.2-2.3. By diffusing into the hydrogel, the osmolyte lowers the osmotic pressure difference, thereby inducing swelling of the hydrogel, lowering the solvent quality at the same time. This causes compression of the hydrogel. The competition between these two effects determines the measured volumetric response of the hydrogel particle at long time scales, see the middle row of Figure 2.4 for an illustration. For the dextran $70 \mathrm{k}$ and PEG 20k the decrease in osmotic pressure difference prevails, causing additional slow 
Table 2.3: Material properties based on the model fits displayed in Figure 2.2 to Figure 2.3. The upper and lower sections of the Table display the swelling and compression cases, respectively. The standard error for each material parameter is given in brackets. We assume all fitted parameters to be uncorrelated.

\begin{tabular}{lcccc} 
Solution & $\begin{array}{c}\Pi \\
{[\mathrm{kPa}]}\end{array}$ & $\begin{array}{c}K \\
{[\mathrm{kPa}]}\end{array}$ & $\begin{array}{c}k \\
{\left[\mathrm{~nm}^{2}\right]}\end{array}$ & $\begin{array}{c}D_{\mathrm{osm}} \\
{\left[\mu \mathrm{m}^{2} / \mathrm{s}\right]}\end{array}$ \\
\hline \hline NaCl only & 0 & 27 & $5.7(0.1)$ & - \\
dextran 70k 8wt\% & 10.4 & $32(1)$ & $3.9(0.1)$ & $1.3(1.6)$ \\
PEG 20k 8wt\% & $64.7(8.6)$ & $51(7)$ & $2.0(0.3)$ & $5.3(2.2)$ \\
PEO 200k 2wt\% & $0.4(0.3)$ & $633(73800)$ & $0.3(31.0)$ & $37.0(16.4)$ \\
PEO 200k 4wt\% & $6.1(0.6)$ & $15(6)$ & $9.6(3.7)$ & $36.7(17.8)$ \\
PEO 200k 5wt\% & $10.8(0.8)$ & $22(13)$ & $5.8(3.5)$ & $46.3(17.5)$ \\
PEO 200k 7.5wt\% & $28.4(1.4)$ & $32(18)$ & $3.3(1.9)$ & $46.4(19.3)$ \\
PEO 200k 10wt\% & $54.1(2.2)$ & $28(4)$ & $3.2(0.5)$ & $26.6(6.8)$ \\
PEO 200k 4wt\%+ & & & & \\
PEG 20k 8wt\% & $70.7(8.6)$ & $30(4)$ & $2.9(0.4)$ & $25.0(4.3)$ \\
\hline dextran 70k 8wt\% & 10.4 & $37(1)$ & $8.7(0.4)$ & $6.0(0.4)$ \\
PEG 20k 8wt\% & $64.7(8.6)$ & $45(6)$ & $4.2(0.7)$ & $3.0(4.7)$ \\
PEO 200k 4wt\% & $6.1(0.6)$ & $33(4)$ & $28.9(5.1)$ & $98.7(17.4)$ \\
PEO 200k 7.5wt\% & $28.4(1.4)$ & $124(11)$ & $13.1(2.2)$ & $79.7(15.3)$ \\
PEO 200k 10wt\% & $54.1(2.2)$ & $161(15)$ & $11.0(2.0)$ & $112.0(23.0)$ \\
\hline \hline
\end{tabular}

swelling in the swelling experiments and slow decompression in the compression experiments, see Figures 2.2B and 2.3B. On the other hand, for PEO 200k the decrease in solvent quality prevails, causing slow compression in the swelling experiments, which results in the observed overshoot, see Figure 2.2A. Also, it causes additional slow compression in the compression experiments, see Figure 2.3A.

For dextran 70k and PEG 20k the diffusion constant in the swelling and compression experiments is consistent and reflects their monodispersity. However, from fits to the data for PEO 200k, after fixing the swelling time scale $\Gamma_{\alpha}$, we obtain significantly higher diffusion coefficients from the swelling experiments than from the corresponding compression experiments, see Table 2.3 and 2.4. Due to the very broad size distribution of PEO 200k, as shown in section 2.6.2 in the Appendix, the fraction of small PEO molecules, which are of approximately the size of dextran 70k and 
Table 2.4: Model parameters and material properties obtained by fitting $P, \Gamma_{f}$ and $B$ for the PEO 200k compression experiments, using $\Gamma_{\alpha}$ from the corresponding swelling experiments shown in Table 2.2. See the dashed lines in Figure 2.3 for the curve fits.

\begin{tabular}{lccc} 
& $4 \mathrm{wt} \%$ & $7.5 \mathrm{wt} \%$ & $10 \mathrm{wt} \%$ \\
\hline \hline$P$ & $5.9(0.2)$ & $9.4(0.5)$ & $16.9(1.1)$ \\
$\Gamma_{\alpha}$ & 0.88 & 0.65 & 0.54 \\
$\Gamma_{f}$ & $2.21(0.83)$ & $0.29(0.08)$ & $0.10(0.76)$ \\
$B$ & $8.5(0.5)$ & $10.4(1.4)$ & $12.3(14.8)$ \\
$\Pi$ & $6.1(0.6)$ & $28.4(1.4)$ & $54.1(2.2)$ \\
$K$ & $10(1)$ & $30(2)$ & $32(2)$ \\
$k$ & $14.1(1.4)$ & $3.5(0.2)$ & $2.8(0.2)$ \\
$D_{\text {osm }}$ & $30.2(11.3)$ & $3.9(1.1)$ & $1.4(10.3)$ \\
\hline \hline
\end{tabular}

PEG 20k, are the first to diffuse into the hydrogel. Therefore, the small fraction is the first to decrease the solvent quality and induce compression of the hydrogel particle. As this compression shows itself as a pronounced overshoot in the hydrogel volume on which we fit our model, we obtain the diffusion constant of the fraction of small PEO molecules from the swelling experiments. Therefore, we find a relatively large apparent diffusion constant in the swelling experiments albeit with a large uncertainty due to the additional compression caused by the fraction of larger PEO molecules, see Table 2.3. In the PEO 200k compression experiments, however, the compression caused by solvent quality changes induced by the low $-M_{\mathrm{w}}$ fraction of PEO 200k coincides with a compression purely due to osmotic pressure differences. As soon as the osmotic pressure difference is balanced by the elasticity of the hydrogel network, the additional slow compression is fully determined by the high $-M_{\mathrm{w}}$ fraction of PEO 200k molecules diffusing into the hydrogel particle, implying a much lower diffusion coefficient, see Table 2.4.

\subsection{Poroelastic model}

In section 2.3 we treated the relaxational dynamics model which showed how the volumetric hydrogel dynamics in concentrated polymer solutions could be explained by considering the competition between changes in the osmotic pressure difference 
between the inside and the outside of the hydrogel particle, and changes in the solvent quality, both governed by the diffusion of osmolyte. To obtain more insight in these competing effects, we consider a dominant-mode model for hydrogel dynamics in polymer solutions drawn from the theory of poroelasticity. We first outline the basic equations of poroelasticity $[27,28,33]$, from which the exact solution for a hydrogel particle in a dilute polymer solution is found, see section 2.6.6-2.6.11 of the Appendix for a detailed derivation. Assuming the long-time dynamics of this solution to be similar to the long-time dynamics in the regime of concentrated polymer solutions, we construct a dominant-mode model for hydrogel swelling and compression.

\subsubsection{Hydrogel dynamics in dilute polymer solutions}

Regarding the hydrogel particle as a superposed solid phase (hydrogel network) and fluid phase (dilute polymer solution), and the osmolyte particles as a constituent of the fluid phase $[131,132]$, the mass conservation equations read in Eulerian form

$$
\begin{aligned}
\frac{\partial \phi_{\mathrm{s}}}{\partial t}+\nabla \cdot\left(\phi_{\mathrm{s}} \mathbf{v}_{\mathrm{s}}\right) & =0 \\
\frac{\partial \phi_{\mathrm{n}}}{\partial t}+\nabla \cdot\left(\phi_{\mathrm{n}} \mathbf{v}_{\mathrm{n}}\right) & =0 \\
\frac{\partial c \phi_{\mathrm{s}}}{\partial t}+\nabla \cdot\left(c \phi_{\mathrm{s}}\left(\mathbf{v}_{\mathrm{n}}+\mathbf{v}_{\text {dif }}\right)\right) & =0
\end{aligned}
$$

where both phases are assumed to be incompressible, $\phi_{\mathrm{s}}$ and $\phi_{\mathrm{n}}$ are, respectively, the volume fractions of the polymer solution and the hydrogel network, $\mathbf{v}_{\mathrm{s}}$ and $\mathbf{v}_{\mathrm{n}}$ are their respective velocities, and $c$ is the concentration of osmolyte particles per unit volume of polymer solution. We idealize the hydrogel particle as being spherically symmetric. Summing equation (2.6) and (2.7), using that $\phi_{\mathrm{s}}+\phi_{\mathrm{n}}=1$, and applying the divergence theorem, we find

$$
\phi_{\mathrm{s}} \mathbf{v}_{\mathrm{s}}+\phi_{\mathrm{n}} \mathbf{v}_{\mathrm{n}}=0
$$

which directly relates the velocity of the polymer solution to the velocity of the hydrogel network.

The advective osmolyte velocity in equation (2.8) equals $\mathbf{v}_{\mathrm{n}}$, because the osmolyte particles are assumed to have much stronger frictional interaction with the hydrogel 
network than with the solvent, i.e., the hydrogel network and the osmolyte particles move advectively as a single complex. Thus, the radius of gyration of an osmolyte particle $R_{\mathrm{g}}$ must be comparable to the mesh size $\zeta$ of the hydrogel network, that is, $R_{\mathrm{g}} \sim \zeta$. Assuming osmolyte diffusion to be Fickian, the velocity of osmolyte particles relative to the hydrogel network $\mathbf{v}_{\text {dif }}$ is given as

$$
c \mathbf{v}_{\text {dif }}=-D_{\mathrm{osm}} \nabla c,
$$

with $D_{\text {osm }}$ the diffusion coefficient of osmolyte inside the hydrogel network.

Assuming mechanical equilibrium and the absence of body forces, the overall force balance for the hydrogel can be written as

$$
\nabla \cdot \boldsymbol{\sigma}=0,
$$

with $\sigma$ the Cauchy stress tensor of the whole hydrogel particle, i.e., of the polymer solution and hydrogel network combined. Using the Terzaghi effective stress [23, 27], we can decompose $\sigma$ as

$$
\sigma_{i j}=\sigma_{i j}^{\prime}-(p+\Pi) \delta_{i j},
$$

with $\boldsymbol{\sigma}^{\prime}$ the elastic stress of the hydrogel network, and $p, \Pi$ and $p+\Pi$ are, respectively, the pneumatic pressure, the total osmotic pressure and the total pressure of the polymer solution [133]. For a dilute polymer solution in the hydrogel network, the total osmotic pressure $\Pi=\Pi_{\mathrm{id}}+\Pi_{\mathrm{exc}}$ is composed of the ideal part $\Pi_{\mathrm{id}}=k_{\mathrm{B}} T c$ and the excess pressure due to hydrogel network-osmolyte interactions $\Pi_{\mathrm{exc}}=k_{\mathrm{B}} T c \rho B_{\mathrm{ns}}$, where $\rho$ is the number density of the hydrogel network and $B_{\mathrm{ns}}$ is the cross-virial coefficient of interactions between the hydrogel network and the osmolyte in the polymer solution, through which the solvent quality can be increased $\Pi_{\text {exc }}>0$ or decreased $\Pi_{\text {exc }}<0$. Darcy's law relates gradients in the pneumatic pressure to the flow of solvent through the hydrogel-osmolyte complex as [27]

$$
\phi_{\mathrm{S}}\left(\mathbf{v}_{\mathrm{s}}-\mathbf{v}_{\mathrm{n}}\right)=-\frac{k}{\eta} \nabla p,
$$

where $k$ is the permeability of the hydrogel-osmolyte complex for the solvent, and $\eta$ is the dynamic viscosity of the solvent.

For dilute polymer solutions, the total osmotic pressure is low, implying the strain in the hydrogel network to be small, and thus the network responds approximately as a 
linear elastic material. Assuming the hydrogel network to be isotropic and homogeneous, the elastic stress of the hydrogel network, relative to the stress-free equilibrium state in osmolyte-free solvent, is written as

$$
\sigma_{i j}^{\prime}=\frac{M}{1-\nu}\left[\nu e_{k k} \delta_{i j}+\left(\frac{1}{2}-\nu\right) e_{i j}\right],
$$

with $e_{i j}=\frac{1}{2}\left(\nabla_{i} U_{j}+\nabla_{j} U_{i}\right)$ the infinitesimal strain tensor, $M=K+4 G / 3$ the longitudinal modulus of the hydrogel network [134], $K$ the bulk modulus, $G$ the shear modulus, and $\nu$ the Poisson ratio. The displacement field $\mathbf{U}(r, t)=U(r, t) \hat{r}$ is radial, with $r \in\left[0, R_{0}\right]$ the radial position in the network and $R_{0}$ the stress-free hydrogel radius.

Mass conservation of osmolyte, equation (2.8), and the overall force balance, equation (2.11) are found as

$$
\begin{aligned}
\frac{\partial c}{\partial t}-D_{\mathrm{osm}} \frac{1}{r^{2}} \frac{\partial}{\partial r} r^{2} \frac{\partial c}{\partial r} & =0, \\
\frac{\eta}{k} \frac{\partial U}{\partial t}-M \frac{\partial}{\partial r} \frac{1}{r^{2}} \frac{\partial}{\partial r} r^{2} U & =-\frac{\partial}{\partial r} k_{\mathrm{B}} T c(1+f),
\end{aligned}
$$

where we defined $f \equiv \rho B_{\mathrm{ns}}$. Equation (2.15) is the standard diffusion equation for osmolyte in the hydrogel network. The displacement field, see equation (2.16), obeys a diffusion-like equation sourced by the gradient in total osmotic pressure. These equations can alternatively be derived from the framework of incompressible mixtures [132].

Due to both short-range repulsive and long-range interactions with the hydrogel network, osmolyte is partitioned between the hydrogel particle and the surrounding polymer solution. The equilibrium partition coefficient $Q$ gives the ratio of the equilibrium concentration of osmolyte in the hydrogel to the osmolyte concentration in the surrounding in equilibrium [125]. As the hydrogel network is homogeneous, the osmolyte concentration at the hydrogel surface equals the equilibrium concentration for all times

$$
c(r, t)=Q c_{0}, \text { at } r=R_{0},
$$

with $c_{0}$ the concentration of osmolyte in the surrounding polymer solution. This concentration can be considered constant, because experimentally the microgel dynamics were shown to be independent of the velocity of the polymer solution [120]. 
Similarly, the Aquapearl particles were put on a rollerbank in a bath of polymer solution to ensure a constant outside osmolyte concentration, see section 2.6.1 of the Appendix. Initially, no osmolyte is present in the hydrogel

$$
c(r, t)=0, \text { at } t=0,
$$

Force balance requires the total pressure of the surrounding polymer solution to equal the total radial stress at the hydrogel surface, i.e., $\sigma_{r r}\left(R_{0}, t\right)=-\left(p_{0}+\Pi_{0}\right)$, with $\sigma_{r r}$ given by equation (2.12), $p_{0}$ the pneumatic pressure of the outside polymer solution and $\Pi_{0}=k_{\mathrm{B}} T c_{0}$ the osmotic pressure of the outside polymer solution. Using that $\Pi=k_{\mathrm{B}} T c(1+f)$, invoking equation (2.17), and the fact that the hydrogel network is permeable for solvent $p\left(R_{0}, t\right)=p_{0}$, the radial network stress at the hydrogel surface is given as

$$
\sigma_{r r}^{\prime}(r, t)=-k_{\mathrm{B}} T c_{0}(1-Q(1+f)), \text { at } r=R_{0},
$$

which shows that the difference in osmotic pressure between the surface of the hydrogel and the surrounding polymer solution can provide a resultant force on the hydrogel surface. In a swelling experiment, where the hydrogel network swells from its fully dried state, the network is initially compressed relative to its stress-free state. In a compression experiment, on the other hand, the hydrogel particle starts out in the stress-free state, giving as initial condition

$$
U(r, t)=r\left(\frac{R_{\text {init }}}{R_{0}}-1\right), \text { at } t=0,
$$

where $R_{\text {init }}$ is the initial radius of the hydrogel network and $R_{0}$ the osmolyte-free equilibrium radius.

Equation (2.17)-(2.20) provide the necessary boundary conditions to solve equation (2.15) and (2.16). The solution to equation (2.15) can be written, using a Hankel transform [135], as

$$
\frac{c(r, t)}{Q c_{0}}=1-\sum_{k=1}^{\infty} 2(-1)^{k+1} \mathrm{j}_{0}\left(\pi k \frac{r}{R_{0}}\right) \exp \left(-(\pi k)^{2} \frac{t}{\tau_{\mathrm{osm}}}\right),
$$

where $\mathrm{j}_{0}$ is the spherical Bessel function of the first kind of order zero, and $\tau_{\text {osm }} \equiv$ $R_{0}^{2} / D_{\text {osm }}$ is the time for the osmolyte to diffuse completely through the hydrogel. 
Next, we may solve equation (2.16) using another Hankel transform to find

$$
\begin{aligned}
& U(r, t)=\sum_{m=1}^{\infty} \frac{U_{m}(t)}{\beta_{m}^{2} N_{m}} \mathrm{j}_{1}\left(\beta_{m} r / R_{0}\right) \\
& U_{m}(t)=U_{m}^{\mathrm{init}}(t)+U_{m}^{\mathrm{bc}}(t)+\sum_{k=1}^{\infty} U_{k m}^{\mathrm{dif}}(t),
\end{aligned}
$$

where $\beta_{m}$ is the $m$-th positive solution of

$$
2 \frac{1-2 \nu}{1-\nu} \mathrm{j}_{1}\left(\beta_{m}\right)=\beta_{m} \mathrm{j}_{0}\left(\beta_{m}\right)
$$

and $N_{m}$ is the normalization of the $m$-th mode of the network displacement field

$$
N_{m}=\frac{1}{2 \beta_{m}^{4}}\left(\cos \left(2 \beta_{m}\right)-1+\frac{\beta_{m}}{2} \sin \left(2 \beta_{m}\right)+\beta_{m}^{2}\right) .
$$

The effect of swelling from an initially compressed or swollen state is given as

$$
\frac{U_{m}^{\mathrm{init}}(t)}{R_{0}}=\frac{R_{\text {init }} / R_{0}-1}{\left(3 \mathrm{j}_{1}\left(\beta_{m}\right)-\beta_{m} \mathrm{j}_{0}\left(\beta_{m}\right)\right)^{-1}} \exp \left(-\beta_{m}^{2} \frac{t}{\tau_{\mathrm{n}}}\right)
$$

where $\tau_{\mathrm{n}} \equiv R_{0}^{2} \eta / k M$ is the typical time for the hydrogel network to swell or compress. The effect of force balance at the hydrogel surface is

$$
\frac{U_{m}^{\mathrm{bc}}(t)}{R_{0}}=-\frac{(1-(1+f) Q) \Pi_{0}}{M} \frac{1-\exp \left(-\beta_{m}^{2} t / \tau_{\mathrm{n}}\right)}{\left(\mathrm{j}_{1}\left(\beta_{m}\right)\right)^{-1}},
$$

and the contribution of the $k$-th mode of osmolyte diffusion to the $m$-th displacement mode is given as

$$
\frac{U_{k m}^{\mathrm{dif}}(t)}{R_{0}}=-(1+f) Q \frac{\Pi_{0}}{M} \frac{2 \sin \left(\beta_{m}\right)}{(\pi k)^{2}-\beta_{m}^{2}} \Gamma_{k m}(t)
$$

where

$$
\Gamma_{k m}(t)=\frac{\exp \left(-(\pi k)^{2} t / \tau_{\mathrm{osm}}\right)-\exp \left(-\beta_{m}^{2} t / \tau_{\mathrm{n}}\right)}{1-(\pi k)^{2} \tau_{\mathrm{n}} / \beta_{m}^{2} \tau_{\mathrm{osm}}} .
$$

Equation (2.26) shows that the effect of swelling from a pre-stressed state is determined by the hydrogel network diffusion time $\tau_{\mathrm{n}}$. Changes in the equilibrium volume 
due to partitioning of osmolyte and changes in solvent quality, see equation (2.27), are also mediated on the network diffusion time scale. The effect of osmolyte diffusion in the hydrogel network, see equation (2.28) and (2.29), generates bi-exponential temporal effects, because the gradient of the osmotic pressure in the hydrogel network, varying on the time scale of osmolyte diffusion $\tau_{\text {osm }}$, causes stress gradients in the network.

\subsubsection{Dominant-mode model}

Given the hydrogel dynamics for hydrogel particles in dilute polymer solutions, we construct a dominant-mode model for hydrogel dynamics in concentrated polymer solutions from the following considerations.

If the time scale of network diffusion is either smaller or comparable to that of osmolyte diffusion, i.e., $\tau_{\mathrm{n}} \lesssim \tau_{\text {osm }}$, the first mode in equation (2.21)-(2.29), with $k=m=1$, is dominant on time scale $\tau_{\text {osm }}$ in the long-time limit; all other modes decay much faster. Thus, the first mode is sufficient to understand the long-time effects of the interplay between osmolyte diffusion and hydrogel (de)swelling.

For a concentrated polymer solution, compared to a dilute polymer solution, the volume change in the hydrogel is expected to be large, because of the high osmotic pressure of the polymer solution, implying that the permeability $k$, the bulk modulus $K$ and the osmolyte diffusion coefficient $D_{\text {osm }}$ may vary appreciably over time. We do not expect them to vary over an order of magnitude, however, because if the hydrogel volume decreases, for example, by $75 \%$, the permeability is expected to decrease by $60 \%$, as approximately $V(t) / V_{\text {init }}=\left(\zeta(t) / \zeta_{\text {init }}\right)^{3}$ and $k(t) \propto \zeta^{2}(t)$, with $\zeta(t)$ the time-varying average mesh size of the hydrogel network. The permeability is usually determined with relative uncertainties in the order of $10 \%$, see section 2.6 .5 of the Appendix, and similar uncertainties hold for the bulk modulus [120] and the osmolyte diffusion coefficient [128]. Therefore, we assume the variance of the material parameters does not give rise to qualitatively different hydrogel-osmolyte dynamics. This does not necessarily imply that the dilute-solution hydrogel dynamics of section 2.4.1 suffices to describe the hydrogel dynamics in concentrated polymer solutions, however, because for concentrated polymer solutions the osmolyte must be described as an independent phase instead of a constituent of the fluid phase. 
As a closed-form approximation to the hydrogel dynamics in concentrated polymer solutions, we propose the dominant mode of the exact solution, equation (2.21)(2.29), but now regarding $K, k$ and $D_{\text {osm }}$ as effective constants. Using only the dominant mode amounts to blurring of the hydrogel boundary, because for $t \rightarrow 0$, the gradient in the osmolyte concentration at the hydrogel surface remains finite, contrary to what equation (2.17) and (2.18) imply.

For a dilute polymer solution, the total osmotic pressure of the polymer solution is the sum of the ideal contribution, which equals the osmotic pressure of the surrounding polymer solution, and an excess contribution due to osmolyte-network interactions. Therefore, in the dominant-mode model we replace the total osmotic pressure in the dominant mode of equation (2.22)-(2.28) by the sum of the osmotic pressure of the surrounding concentrated polymer solution $\Pi_{0}$ and a pressure quantifying the solvent-quality change $\Pi_{\mathrm{exc}}$, giving the radius of the hydrogel relative to the stressfree state $\Delta R(t)$ as

$$
\Delta R(t)=\Delta R^{\mathrm{init}}(t)+\Delta R^{\mathrm{bc}}(t)+\Delta R^{\mathrm{dif}}(t),
$$

with the contribution due to initial compression or swelling given by

$$
\Delta R^{\text {init }}(t)=\frac{k}{\eta} \frac{M\left(1-R_{\text {init }} / R_{0}\right)}{R_{0}} C^{\text {init }}(\nu) \frac{\exp \left(-t / t_{\mathrm{n}}\right)}{-1 / t_{\mathrm{n}}},
$$

where $t_{\mathrm{n}} \equiv R_{0}^{2} \eta / k M \beta_{1}^{2}$ is the exponential relaxation time for hydrogel swelling or compression. Force balance at the hydrogel surface provides

$$
\Delta R^{\mathrm{bc}}(t)=-\frac{k}{\eta} \frac{(1-Q) \Pi_{0}-Q \Pi_{\mathrm{exc}}}{R_{0}} C^{\mathrm{bc}}(\nu) \frac{1-\exp \left(-t / t_{\mathrm{n}}\right)}{1 / t_{\mathrm{n}}},
$$

which determines the equilibrium volume of the hydrogel immersed in polymer solution. The effect of osmolyte diffusion results in

$$
\Delta R^{\mathrm{dif}}(t)=-\frac{k}{\eta} \frac{Q\left(\Pi_{0}+\Pi_{\mathrm{exc}}\right)}{R_{0}} C^{\mathrm{dif}}(\nu) \frac{e^{-t / t_{\mathrm{osm}}}-e^{-t / t_{\mathrm{n}}}}{1 / t_{\mathrm{n}}-1 / t_{\mathrm{osm}}},
$$

which is proportional to the maximum osmotic pressure of the polymer solution in the hydrogel network and where $t_{\mathrm{osm}} \equiv R_{0}^{2} / D_{\mathrm{osm}} \pi^{2}$ is the exponential relaxation time for osmolyte diffusion.

The proportionality constants in equation (2.31)-(2.33), are given as $C^{\text {init }}(\nu)=$ $\mathrm{j}_{1}\left(\beta_{1}\right)\left(3 \mathrm{j}_{1}\left(\beta_{1}\right)-\beta_{1} \mathrm{j}_{0}\left(\beta_{1}\right) / N_{1}, \quad C^{\mathrm{bc}}(\nu)=\mathrm{j}_{1}^{2}\left(\beta_{1}\right) / N_{1}\right.$ and $C^{\text {dif }}(\nu)=$ 
$2 \mathrm{j}_{1}\left(\beta_{1}\right) \sin \left(\beta_{1}\right) / N_{1}\left(\pi^{2}-\beta_{1}^{2}\right)$. For $-0.08<\nu \leq 1 / 2$, we can approximate $\beta_{1}$ as

$$
\beta_{1}=\pi\left(1-\frac{2(1-2 \nu)}{\pi^{2}-4+\nu\left(\pi^{2}+8\right)}\right),
$$

within an accuracy of $1 \%$. To first order in $x \equiv 1-\beta_{1} / \pi$ the proportionality constants read $C^{\text {init }}(\nu) \approx 6+\left(18-2 \pi^{2}\right) x, C^{\text {bc }}(\nu) \approx 2+6 x$ and $C^{\text {dif }}(\nu) \approx 2+3 x$, a particularly useful approximation for polyacrylamide hydrogels having a Poisson ratio of about 0.48 [136].

Pre-stress in the hydrogel network, see equation (2.31), and the force balance at the hydrogel boundary, see equation (2.32), cause swelling or compression on time scale $t_{\mathrm{n}}$ to the equilibrium state of the hydrogel, which is determined by the balance between the resultant compressive force $(1-Q) \Pi_{0}$ over the hydrogel surface and the solvent quality pressure $Q \Pi_{\text {exc }}$ at the hydrogel surface. For $Q=0$ the hydrogel is compressed in equilibrium relative to the stress-free state due to the osmotic pressure of the surrounding polymer solution $\Pi_{0}>0$. If the solvent quality increases, however, such that $\Pi_{0}(1-Q)<Q \Pi_{\text {exc }}$, the hydrogel can swell relative to the stress-free state.

In case osmolyte diffusion is slow, i.e, $t_{\mathrm{osm}} \gtrsim t_{\mathrm{n}}$, additional (de)swelling can occur on time scale $t_{\mathrm{osm}}$, depending on the sign of $\Pi_{0}+\Pi_{\mathrm{exc}}$, see equation (2.33). This slow (de)swelling stems from two competing effects due to osmolyte diffusion. On the one hand, the gradient in the osmotic pressure $\Pi_{0}$ vanishes, which decreases the compressive force on the hydrogel particle, thus inducing swelling. On the other hand, the gradient in solvent quality pressure $\Pi_{\text {exc }}$ vanishes. The latter induces either swelling if the osmolyte increases the solvent quality, $\Pi_{\mathrm{exc}}>0$, or compression if the solvent quality is decreased $\Pi_{\mathrm{exc}}<0$. Therefore, for a strong decrease in solvent quality, with $\Pi_{0}+\Pi_{\mathrm{exc}}<0$, an overshoot in a swelling experiment can occur, see Figure 2.6. Similarly, if $\Pi_{0}+\Pi_{\text {exc }}>0$, an undershoot in a compression experiment is possible, see Figure 2.7. In comparison with the relaxational dynamics model of section 2.3 we find an explicit criterion which determines whether an overshoot or undershoot may occur, see the middle row of Figure 2.4 for an illustration of a strong (solid curve) and weak (dashed curve) decrease in solvent quality.

The velocity of the hydrogel surface at $t=0, v_{0}$, is given by a global form of Darcy's law $v_{0} \propto k \Delta P / \eta R_{0}$, which stems from the approximative nature of the dominantmode model. The pressure difference $\Delta P$ in equation (2.31) is proportional to the 


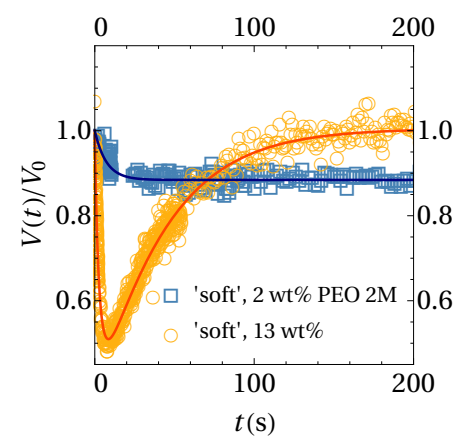

(D)

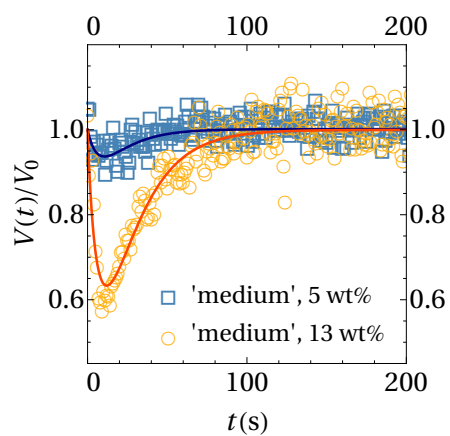

(E)

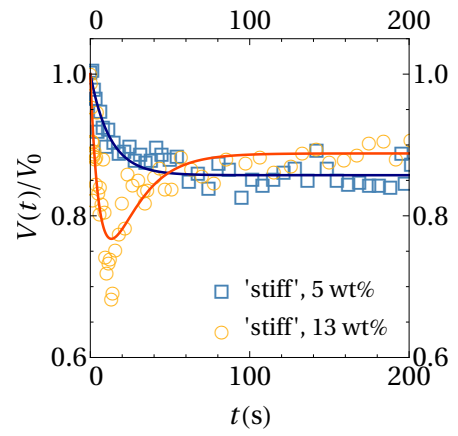

(F)

Figure 2.5: The ratio of the volume $V(t)$ of 'soft' (D), 'medium' (E), and 'stiff' (F) microgels to their original volume $V_{0}$ is given as a function of time $t$ after water is replaced by a dextran $70 \mathrm{k}$ polymer solution, see Figure $2.1 \mathrm{~A}-\mathrm{C}$, unless specified otherwise. Replacing water by a PEO $2 \mathrm{M}$ polymer solution (D) shows that the non-monotonic response is associated with osmolyte diffusion into the hydrogel particle. The solid lines are fits to the dominant-mode model, with parameters displayed in Table 2.5.

stress initially present in the hydrogel network, in equation (2.32) it is proportional to the osmotic pressure difference between the inside and the outside of the hydrogel in equilibrium, and in equation (2.33) it is proportional to the equilibrium osmotic pressure of the polymer solution in the hydrogel network.

To test the validity of the dominant-mode model, we compare it to measurements of the hydrogel volume in the swelling and compression experiments described in section 2.2.

\subsubsection{Comparison to experiment}

To test the dominant-mode model proposed in the previous section, we use equation (2.30)-(2.33) to obtain estimates for the (effective) osmolyte diffusion constant $D_{\text {osm }}$, and both the bulk modulus $K \equiv M(1+\nu) / 3(1-\nu)$ and the permeability $k$ of the hydrogel particles, with $M$ the longitudinal modulus and $\nu \approx 0.48$ the Poisson ratio [136, 137], by fitting the dominant-mode model to the measured volumetric response of both the microgels and the Aquapearl hydrogels, see Figure 2.5-2.7. Also, we find an estimation for the change in solvent quality as the excess osmotic pressure $\Pi_{\text {exc }}$ of the osmolyte in the hydrogel network, similar to the $B$ parameter in 


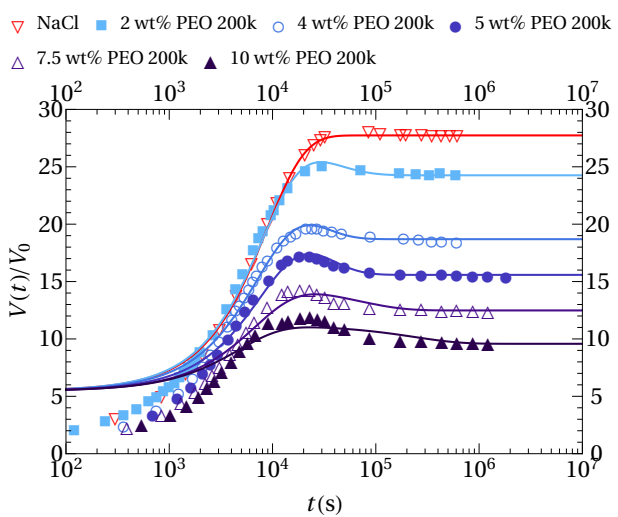

(A)

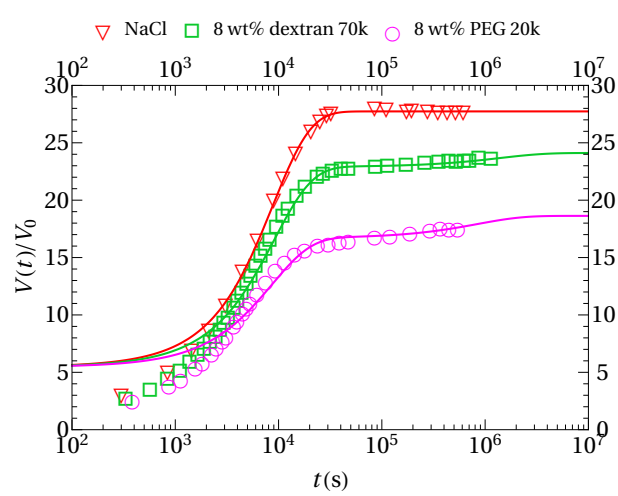

(B)

Figure 2.6: The normalized volume $V(t) / V_{0}$ as a function of time $t$ of initially dry Aquapearl hydrogel particles for different PEO 200k concentrations exhibiting an overshoot (A), and for dextran 70k and PEG 20k exhibiting no overshoot (B). The solid lines are fits of the dominant-mode model, with material parameters displayed in the upper half of Table 2.6.

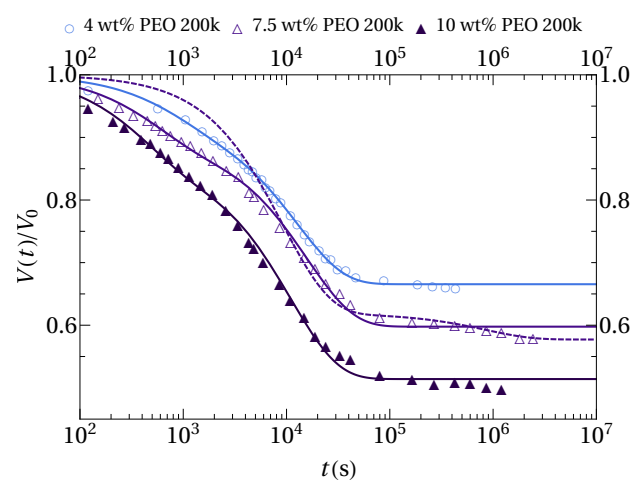

(A)

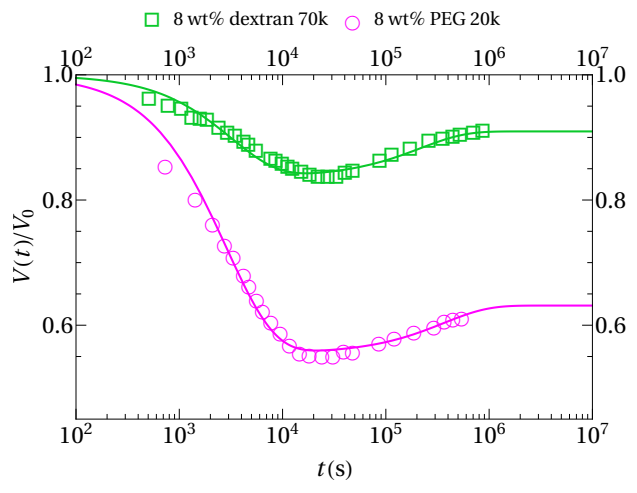

(B)

Figure 2.7: The normalized volume $V(t) / V_{0}$ as a function of time $t$ of Aquapearl hydrogel particles in different PEO 200k concentrations, exhibiting no undershoot (A), and for dextran 70k and PEG 20k exhibiting an undershoot (B). The solid lines are fits of the relaxational dynamics model, with material parameters displayed in the lower half of Table 2.6. The dashed line in (A) is a fit of the $7.5 \mathrm{wt} \%$ PEO 200k experiment which is forced to take into account the final stage of slow deswelling, see Table 2.7 for fit parameters.

the relaxational dynamics model of section 2.3. We estimate the equilibrium partition coefficient $Q$ either from literature for the microgels [126] or by modeling the available volume in the hydrogel network as spherical pores for the Aquapearls, see 
Table 2.5: Material properties from the fits with the dominant-mode model on 'soft', 'medium' and 'stiff' microgel particles, some of which are displayed in Figure 2.5. The standard error for each derived material parameter is given in brackets. We assumed all fitted parameters to be uncorrelated. The value for the bulk modulus $K$ without uncertainty specification has been taken from Capillary Micromechanics measurements [120].

\begin{tabular}{lcccc} 
Solution & $\begin{array}{c}K \\
{[\mathrm{kPa}]}\end{array}$ & $\begin{array}{c}k \\
{\left[\mathrm{~nm}^{2}\right]}\end{array}$ & $\begin{array}{c}D_{\mathrm{osm}} \\
{\left[\mu \mathrm{m}^{2} / \mathrm{s}\right]}\end{array}$ & $\begin{array}{c}\Pi_{\text {exc }} \\
{[\mathrm{kPa}]}\end{array}$ \\
\hline \hline Medium 13wt\% & $8.5(1.7)$ & $0.22(0.02)$ & $5.4(1.0)$ & $238.0(0.4)$ \\
Medium 13wt\% & $6.3(1.1)$ & $0.25(0.03)$ & $7.1(1.0)$ & $234.0(0.8)$ \\
Medium 5wt\% & 12 & $0.23(0.04)$ & $4.3(0.5)$ & $34.4(0.3)$ \\
Medium 5wt\% & 12 & $0.13(0.02)$ & $3.5(0.3)$ & $-34.4(0.3)$ \\
Soft 13wt\% & $2.0(0.1)$ & $0.53(0.01)$ & $12.7(0.3)$ & $238.0(0.1)$ \\
Soft PEG 2M & $9.1(0.1)$ & $0.58(0.04)$ & - & - \\
Stiff 5wt\% & 17 & $0.18(0.11)$ & $7.5(293.0)$ & $3.0(4.8)$ \\
Stiff 13 wt\% & 17 & $0.13(0.01)$ & $5.9(0.6)$ & $1280.0(10.8)$ \\
\hline \hline
\end{tabular}

section 2.3.1. The osmotic pressure of the polymer solutions $\Pi_{0}$ as a function of the concentration of osmolyte are known from literature for the 70k dextran [123], the PEG 20k [129] and the PEO 200k polymer solutions, for the latter see section 2.6.3 of the Appendix. Using the Mathematica function NonlinearModelFit, the dominant-mode model is fitted to the volumetric measurements, see Figure 2.5-2.7. In case the fit routine did not converge, the value of $K$ or $k$ was fixed from the estimated value determined with, respectively, CM or a conventional permeability test, see section 2.6.4 and 2.6.5 of the Appendix. See Table 2.5 and 2.6 for a summary of, respectively, the fit parameters for the microgel and the Aquapearl particles.

As explained in section 2.4.2, the dominant-mode model contains the effective values for the material parameters $K, k, D_{\text {osm }}$ and $\Pi_{\text {exc }}$. Nevertheless, we do expect the fitted values to give the typical order of magnitude and trends in the material parameters, e.g., the magnitude of the solvent quality pressure $\Pi_{\text {exc }}$ is expected to increase with osmolyte concentration. For the microgels, the order of magnitude of the bulk modulus $K$ agrees with independent measurements from Capillary Micromechanics (CM) [120], where the bulk modulus of the medium particles was found as $K=13(5) \mathrm{kPa}$, with the uncertainty in brackets. The permeability $k$ decreases with increasing bulk modulus, which is to be expected, as a higher bulk modulus implies 
Table 2.6: Material properties based on the model fits displayed in Figure 2.6 to Figure 2.7. The upper and lower sections of the Table display the swelling and compression cases, respectively. The standard error for each derived material parameter is given in brackets. We assumed all fitted parameters to be uncorrelated. The value for the bulk modulus $K$ and the permeability $k$ without uncertainty specification has been taken from Capillary Micromechanics measurements and permeability measurements, see section 2.6.4 and 2.6.5 of the Appendix.

\begin{tabular}{lcccc} 
Solution & $\begin{array}{c}K \\
{[\mathrm{kPa}]}\end{array}$ & $\begin{array}{c}k \\
{\left[\mathrm{~nm}^{2}\right]}\end{array}$ & $\begin{array}{c}D_{\mathrm{osm}} \\
{\left[\mu \mathrm{m}^{2} / \mathrm{s}\right]}\end{array}$ & $\begin{array}{c}\Pi_{\text {exc }} \\
{[\mathrm{kPa}]}\end{array}$ \\
\hline \hline Saline & 27 & $7.5(0.5)$ & - & - \\
dextran 70k 8wt\% & $35(5)$ & $5.5(1.0)$ & $1.0(16.1)$ & $-6.8(41.5)$ \\
PEG 20k 8wt\% & $86(8)$ & $2.2(0.5)$ & $1.7(36.8)$ & $-46.3(297.0)$ \\
PEO 200k 2wt\% & 27 & $7.9(0.5)$ & $45.1(53.8)$ & $-17.6(4.2)$ \\
PEO 200k 4wt\% & 27 & $7.6(0.5)$ & $82.1(35.1)$ & $-38.6(3.6)$ \\
PEO 200k 5wt\% & 27 & $6.9(0.8)$ & $62.4(28.3)$ & $-49.7(4.3)$ \\
PEO 200k 7.5wt\% & $30(2)$ & 8 & $15.9(28.3)$ & $-45.9(9.7)$ \\
PEO 200k 10wt\% & $43(2)$ & 8 & $4.8(14.3)$ & $-78.0(24.7)$ \\
\hline dextran 70k 8wt\% & $37(1)$ & $10.5(0.6)$ & $6.4(1.8)$ & $-4.0(0.5)$ \\
PEG 20k 8wt\% & $75(1)$ & $5.3(0.2)$ & $3.9(4.8)$ & $-46.2(11.3)$ \\
PEO 200k 4wt\% & $44(3)$ & $37.2(3.0)$ & $98.8(3.2)$ & $-73.2(5.8)$ \\
PEO 200k 7.5wt\% & $153(10)$ & $16.3(1.4)$ & $78.2(4.4)$ & $-315.0(25.4)$ \\
PEO 200k 10wt\% & $219(16)$ & $14.4(1.3)$ & $109.0(7.0)$ & $-562.0(49.0)$ \\
\hline \hline
\end{tabular}

a smaller cross-linker to cross-linker distance and therefore a smaller mesh size and permeability. The odd one out, however, is the PEG 2M experiment on soft particles, which disagrees with the $13 \mathrm{wt} \%$ dextran $70 \mathrm{k}$ experiment on the bulk modulus of the soft particles. The osmolyte diffusion constant is expected to be a fraction of its value in the surrounding polymer solution [138]. Indeed, the fitted values for $D_{\text {osm }}$ are a fraction of the mutual diffusion coefficient in a $13 \mathrm{wt} \%$ dextran $70 \mathrm{k}$ solution, $D_{\text {osm }}=6 \cdot 10^{1} \mu \mathrm{m}^{2} \mathrm{~s}^{-1}$ [128]. Finally, the solvent quality pressure $\Pi_{\text {exc }}$ increases with the concentration of osmolyte, as expected.

For the Aquapearl swelling experiments the fitted permeability agrees with independent measurements which indicate $k=8(5) \mathrm{nm}^{2}$, see section 2.6 .5 of the appendix. Moreover, the bulk modulus agrees to that obtained by CM, see section 2.6.4 of the 
Table 2.7: Fitted material properties from the PEO 200k $7.5 \mathrm{wt} \%$ experiment where the data points are weighted proportional to their time coordinate, see the dashed line in Figure 2.7 for the corresponding curve fit. The extracted diffusion coefficient corresponds, most probably, to a high molecular weight species of the osmolyte.

\begin{tabular}{lcccc} 
Solution & $\begin{array}{c}K \\
{[\mathrm{kPa}]}\end{array}$ & $\begin{array}{c}k \\
{\left[\mathrm{~nm}^{2}\right]}\end{array}$ & $\begin{array}{c}D_{\mathrm{osm}} \\
{\left[\mu \mathrm{m}^{2} / \mathrm{s}\right]}\end{array}$ & $\begin{array}{c}\Pi_{\text {exc }} \\
{[\mathrm{kPa}]}\end{array}$ \\
\hline \hline PEO 200k 7.5 wt\% & $40(1)$ & $3.2(0.2)$ & $1.6(0.4)$ & $-41(1)$
\end{tabular}

appendix, except for the PEG 20k swelling experiment. The osmolyte diffusion coefficients all have a rather large uncertainty. For the PEO 200k experiments this is probably caused by polydispersity in osmolyte size, see section 2.6.2 of the Appendix and the discussion in section 2.3.2. For the dextran 70k and PEG 20k swelling experiments the uncertainty may be connected to the fact that they do not exhibit an overshoot, thereby making the effect of osmolyte diffusion less pronounced. Finally, the PEO 200k swelling experiments show that the excess osmotic pressure increases with the osmolyte concentration, as expected.

The compression experiments on dextran 70k and PEG 20k roughly agree with the appurtanent swelling experiments, showing the robustness of the dominant-mode model. The PEO 200k compression experiments, however, show anomalously high values for all four fit parameters. In section 2.6.2 of the Appendix we establish that the PEO 200k osmolyte consists of a wide range of species having different sizes. As the different species have different diffusion constants and, possibly, different effects on the solvent quality, a complicated evolution of the volume of the hydrogel particle is to be expected. The dominant-mode model assumes only a single size of osmolyte molecules, however, implying the fitted material parameters to reflect a kind of average effect of all osmolyte species. As a result, the solid line fits in Figure 2.7A show, for example, that the slow deswelling for $t \gtrsim 10^{5} \mathrm{~s}$ is effectively ignored, for the model fit equilibrates at $t \sim 10^{5} \mathrm{~s}$. Indeed, if we force the fit routine to take into account the final deswelling process by weighing each data point proportionally to the time coordinate, and hence giving more weight to the long-time data, the fit of, for example, the $7.5 \mathrm{wt} \%$ PEO 200k compression experiment provides credible values for all four fit parameters, see the dashed line in Figure 2.7A and Table 2.7 for the fit parameters. The fitted diffusion constant, most probably, pertains to a high molecular weight species of PEO 200k, the excess osmotic pressure agrees with the appurtenant 
swelling experiment, and the bulk modulus and permeability of the hydrogel particle agree with their independent measurement, see section 2.6.4 and 2.6.5 of the Appendix. Moreover, the polydispersity in the PEO 200k osmolyte probably causes the large value and uncertainty of the fitted diffusion constant for the PEO 200k swelling experiments, similar as with the relaxational dynamics model of section 2.3 . The low molecular weight species cause a fast decrease in solvent quality, giving rise to the overshoot in hydrogel volume, see Figure 2.6A.

\subsection{Conclusions and discussion}

We construct two models to account for the swelling and compression of hydrogels in polymer solutions: a semi-phenomenological relaxational dynamics model, which minimizes the free energy of the hydrogel particle, and, using the theory of poroelasticity, a dominant-mode model. The dominant-mode model is derived through a dominant mode assumption: the long-time hydrogel dynamics in concentrated polymer solutions is assumed to be similar to that in dilute polymer solutions.

The equilibrium volume of the hydrogel is shown to be determined by the balance between the osmotic compressive force and the solvent quality pressure at the hydrogel surface. Diffusion of osmolyte results in non-monotonic changes in hydrogel volume. On the one hand, osmolyte diffusion causes a gradual decrease in compressive force on the hydrogel particle, resulting in initial compression and subsequent reswelling. On the other hand, depending on whether the osmolyte increases or decreases the solvent quality for the hydrogel network, it can cause swelling or compression at the time scale of osmolyte diffusion. The dominant-mode model provides, contrary to the relaxational dynamics model, an explicit criterion of whether an overshoot or undershoot can occur in the hydrogel volume, depending on the magnitude of the change in solvent quality for the hydrogel network due to the presence of the osmolyte. Also, the dominant-mode model shows that the spatial-temporal effect of osmolyte diffusion may cause non-monotonic evolution of the hydrogel volume. In the relaxational dynamics model, however, where only temporal feedback between osmolyte diffusion and hydrogel swelling was taken into account, non-monotonicity can also be accounted for, showing the temporal effect of osmolyte diffusion to be crucial to understand non-monotonic (de)swelling of hydrogels. 
Comparison of both models to experiment provides reasonable estimations of the bulk modulus and the permeability of the hydrogel network, the osmolyte diffusion constant and the solvent quality change due to the presence of osmolyte. Therefore, the dominant mode assumption, which assumes the long-time hydrogel dynamics in dilute and concentrated polymer solutions to be similar, seems justified. One could argue that this is to be expected, since the material parameters do not vary strongly during the majority of the time-evolution of the hydrogel volume. What is unexpected, however, is the fact that the dominant-mode model also accurately describes the short-time response of the microgel particles, see Figure 2.5. We hypothesize this to be due to a cancellation of errors between the idealized description of the experimental setup and the neglect of higher order modes in the exact solution.

In this study we focused on osmolyte diffusion and the appurtenant effects of its osmotic pressure on the swelling and compression of hydrogels. The dominantmode model can be extended to account for changes in the external $\mathrm{pH}$ and temperature, however, as their spacial-temporal propagation is also governed by a diffusion equation. Therefore, we expect the dominant-mode model to have wider applicability. 


\subsection{Appendix}

\subsubsection{Roller bank}

We measure the weight of a hydrogel particle, which has been swollen in a saline solution for at least a week, as a function of time, after we submerge it in a PEO solution. For samples exposed to a polymer solution at rest, without using the roller bank, a distinct delay in compression is observed. We hypothesize that this has to do with the formation of a depletion zone around the particle. Changing the settings of the roller bank might also influence this process, but we have not investigated this in detail.

\subsubsection{Dynamic Light Scattering on PEO so- lutions}

To obtain more information on the molecular weight distribution of the PEO, we perform dynamic light scattering (DLS) experiments. For a polymer with a uniform molecular weight distribution we would expect a single exponential decay of the electric field correlation function $g_{1}=$ $\left[g_{2}-1\right]^{0.5}$ as a function of time [139], see Figure 2.9. Our measured $g_{1}$, however, clearly does not decay as a single exponential, instead exhibiting a broad distribution of relaxation times. This indicates that the PEO polymer used in our experiments exhibits a broad size distribution. The inset of Figure 2.9 shows the corresponding distribution in hydrodynamic radius, as estimated from the temporal correlation function,

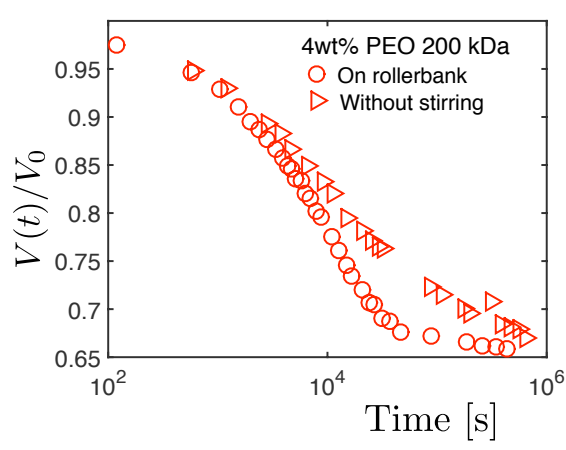

Figure 2.8: Normalized weight as a function of time for a sample on and off the roller bank.

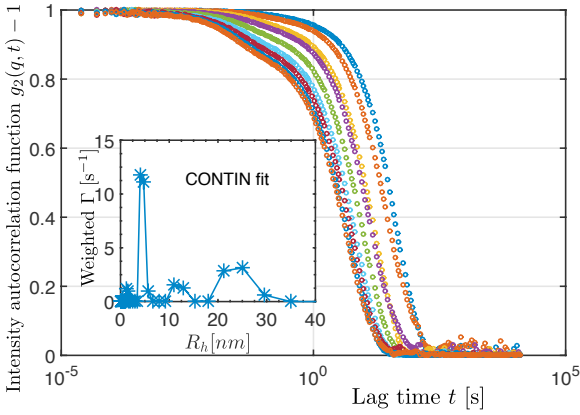

Figure 2.9: Dynamic light scattering data. Electric field correlation function $g_{2}(t)-1$ as a function of lag time $t$, measured at scattering angles ranging from 30 to 120 degrees. The shape of the correlation functions is clearly broader than single exponential, indicating that the PEO does not exhibit a narrow molecular weight distribution. Inset: Distribution of radius of hydration, as extracted from a CONTIN fit to the measured correlation functions. 
using a CONTIN fitting algorithm [140] as implemented in the freely available AfterALV software (AfterALV 1.0d, Dullware Inc, The Netherlands).

\subsubsection{Osmotic pressure PEO}

We measure the osmotic pressure of the PEO solutions as a function of concentration by dialysis against dextran solutions, for which the osmotic pressure has previously been measured in detail [123]. We enclose $2 \mathrm{wt} \%$ PEO solutions into dialysis bags and place them into baths of dextran solutions of various concentrations with corresponding osmotic pressures ranging from $0.9 \mathrm{kPa}$ to $40.7 \mathrm{kPa}$. These baths are then allowed to equilibrate for a period of 1 week. After this period we assume that the osmotic pressure of PEO inside the bag matches that of dextran in the bath outside. Depending on whether the initial osmotic pressure in the PEO solution is larger or smaller than that in the surrounding dextran solution, the concentration within the dialysis bag will have increased or decreased after equilibration, respectively. We neglect changes in concentration in the dextran bath around the dialysis bag, as the volume of the dextran solution far exceeds the volume of the sample in the dialysis bag (ratio 50:1).

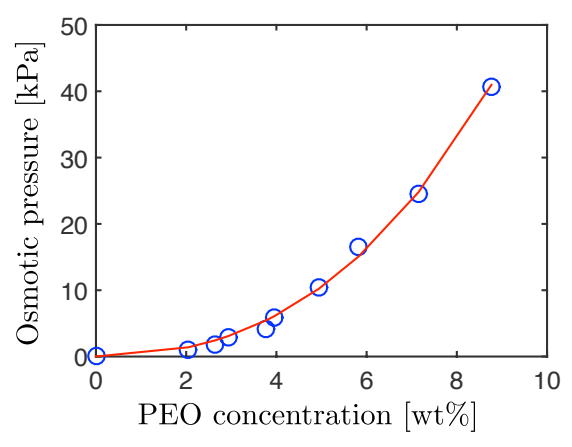

Figure 2.10: Osmotic pressure as a function of concentration for PEO solutions, as determined from dialysis against dextran solutions. The blue circles are the experimental data, and the red line is our second order polynomial fit.
To determine the concentrations of the equilibrated PEO solutions, we extract the solutions from the dialysis bags and determine their weight both immediately after extraction and after thorough overnight drying on a hotplate, respectively. The PEO weight concentration is then taken as the ratio of the dry weight to the initial weight of the solution. The resulting data points for the different solutions are shown in Figure 2.10 as blue circles. The red line is a second order polynomial fit to the data, which adequately describes the experimental data over the range of concentrations studied. To obtain the osmotic pressure of PEG 20k from literature [129], we also used a second order fit of the measured osmotic pressure in the range of 
0-10 wt \%.

\subsubsection{Capillary Micromechanics}

As a comparison with our osmotic compression experiments, we quantify the mechanical properties of our hydrogel particles using the recently developed Capillary Micromechanics method [122]. The results mainly serve as a validation for our developed model. During Capillary Micromechanics, a particle of interest is flown into a tapered glass capillary of circular cross-section. As the tip of the capillary is smaller in diameter than the particles, the particle gets trapped and subsequently blocks the flow. In this situation, the entire pressure difference applied across the capillary falls off across the trapped particle. The corresponding applied external stress must match the internal elastic

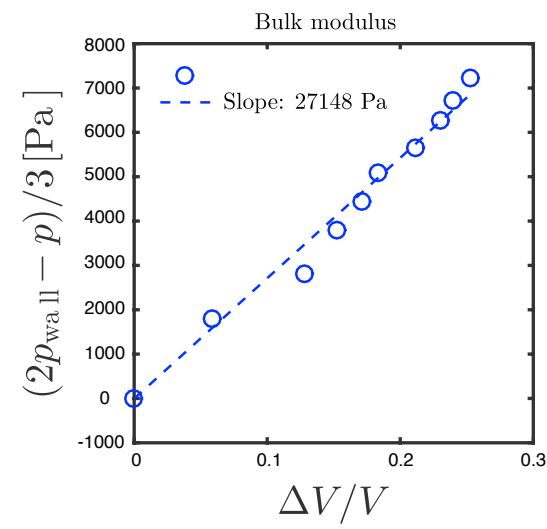

Figure 2.11: Volume change $\Delta V / V$ as a function of the characteristic bulk stress $\left(2 p_{\text {wall }}+p\right) / 3$, as measured using Capillary Micromechanics. stress within the particle, which is a function of the particle's deformation and the elastic moduli of the particle. As the particle changes both its shape as well as its volume in the process, quantifying its deformation enables us to directly extract the full elastic response, including the compressive (bulk) modulus $K$ and the shear elastic modulus $G$. To extract $K$, we quantify the volumetric strain $\Delta V / V$ as a function of the characteristic bulk stress $\sigma_{\text {compr. }} \approx\left[2 \cdot p_{\text {wall }}+p\right] / 3$ applied to the particle, where $p_{\text {wall }}$ is the pressure exerted on the particle at the area of contact between the particle and the wall of the capillary, and $p$ is the pressure drop applied across the capillary. The result for a particle which is swollen in a saline solution for at least a week is depicted in Figure 2.11. From the slope we find $K=27(5) \mathrm{kPa}$, with the fit uncertainty in brackets. 


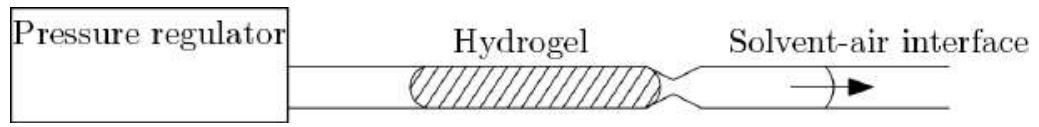

Figure 2.12: Home-built setup to determine the permeability of hydrogel particles.

\subsubsection{Home-built permeability setup}

The permeability $k$ is measured using a home-built setup consisting of a thin-walled glass capillary (TW120-6, WPI, USA) and a pressure regulator (MFC-EZ, Fluigent, Germany). In the capillary we create an hourglass-shaped notch by controlled pulling with a micropipette puller (model P-97, Sutter Instruments, USA). The function of this notch is to prevent the hydrogel from moving forward when pressure is applied to it. To prevent air bubbles in the system, we first fill the capillary with the solvent in which the hydrogel of interest has been swollen. Hereafter we insert the hydrogel by piercing the capillary through the hydrogel, thereby cutting out a cylindrical piece of hydrogel that fits snugly into the capillary. Finally we connect the pressure setup to the capillary. We apply pressures between $0.8 \mathrm{kPa}$ and $4 \mathrm{kPa}$ and track the movement of the solvent-air interface as a function of time after the application of pressure. A

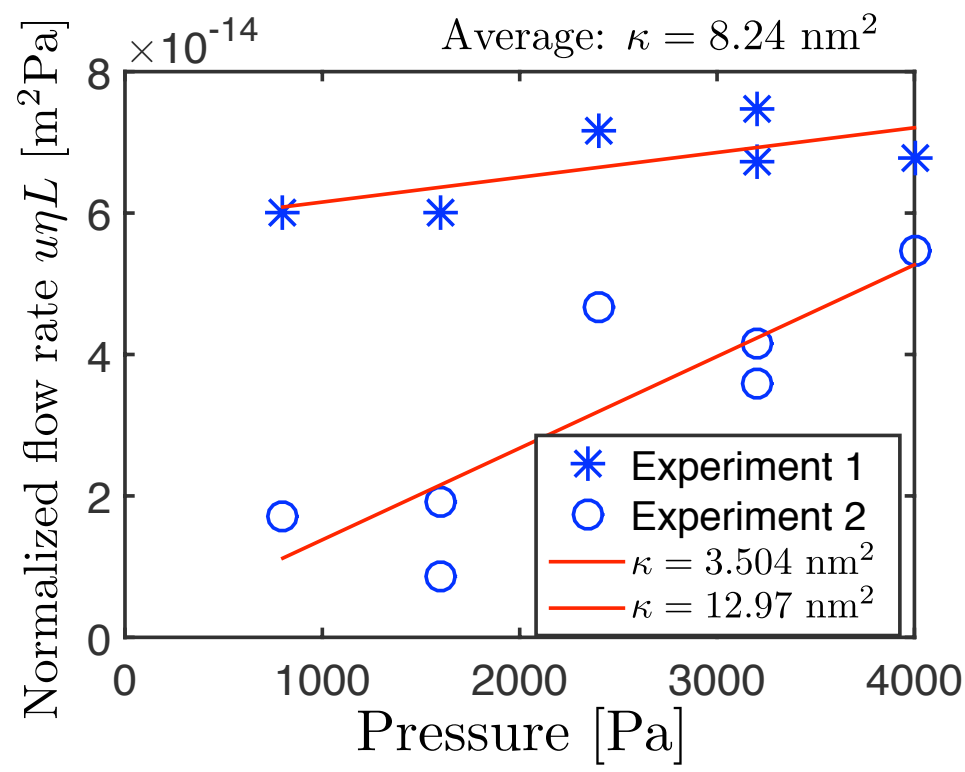

Figure 2.13: Normalized flow rate as a function of applied pressure. The slope indicates the permeability. 
schematic overview of the setup is shown in Figure 2.12. According to Darcy's law, the flow velocity is proportional to the pressure drop $\Delta P$, as $u \eta L=\Delta P k$, with $u$ the fluid flow rate, $\eta$ the dynamic viscosity, and $L$ the length of the porous material over which the pressure difference is applied. To obtain the permeability $k$, we thus plot the scaled flow rate $(u \eta L)$ as a function of the pressure difference $\Delta P$, as shown in Figure 2.13; the permeability can be directly extracted as the slope of the resultant curve. We have plotted the results for two particles. We here obtain permeabilities of $k=8(5) \mathrm{nm}^{2}$, with the uncertainty in brackets. These values are reasonable and within the range of permeabilities previously reported for hydrogels of similar polymer concentration.

\subsubsection{Theoretical background}

In the following sections we give the theoretical background to the poromechanical analysis of section 2.4.1 from which we developed the dominant-mode model. First, we show the general implications of fluid, network and osmolyte mass conservation. Subsequently, we elaborate on the force balance inside the gel and the role herein of the osmotic pressure exerted by the osmolyte molecules. Next, we consider the implications of our assumption of spherical symmetry and formulate the equations of motion (EOM) for a spherically symmetric hydrogel. Finally, we take the limit of hydrogel dynamics in dilute polymer solutions, and give a detailed derivation of the solution to the EOMs in this limit.

\subsubsection{Conservation laws}

We describe the hydrogel as a superposition of two continua: a solid continuum (hydrogel network) and a fluid continuum (dilute polymer solution). Both continua fill the entire space and are made up of infinitely many material particles. Movement of the two is governed by mass conservation, force balance and frictional interactions between the two continua. The osmolyte molecules are assumed to move with and to diffuse through the network because the radius of gyration of the osmolyte molecules $\left(R_{\mathrm{g}} \sim 6 \mathrm{~nm}[141,142]\right)$ is comparable to the pore radius of the network ( $\zeta \sim 30 \mathrm{~nm}$ for the $5 \mathrm{wt} \%$ polyacrylamide mirogels [143]). Therefore, we assume osmolyte molecules to move advectively with the hydrogel network. Moreover, we 
assume the volume fraction of osmolyte molecules to be small compared to the volume fraction of fluid.

In this section we first derive the mass conservation equations in Eulerian form for the network, the fluid and the osmolyte molecules, using the theory of poromechanics as formulated by Coussy [28]. Subsequently, we cast the conservation laws in their Lagrangian form, as this is a more convenient form for making (numerical) computations.

\section{Eulerian framework}

First thing to note is that we assume all pressures (hydrostatic pressure, Terzaghi effective stress and osmotic pressure) to be of such a magnitude that the specific density of both the hydrogel network and the fluid are constant. This is a safe assumption since the bulk modulus of pure water is of the order GPa, whilst the osmotic pressure exerted by the osmolyte molecules is of the order of $\mathrm{kPa}$. The specific density of fluid, for example, is the mass of fluid per unit of fluid volume, as opposed to the average density, which is the mass of fluid per unit of total volume. Furthermore, we do not bother about any temperature inhomogeneities because of the following. The typical time needed for heat to diffuse through the hydrogel $t_{q}$ is of the order $t_{q}=R_{0}^{2} / \alpha$, with $R_{0}$ the radius of the undeformed hydrogel and $\alpha$ the thermal diffusivity of a polyacrylamide hydrogel. Is has been established that $\alpha$ is of the order $10^{-7} \mathrm{~m}^{2} / \mathrm{s}[144,145]$ and in the (de)swelling experiments on microgels $R_{0} \approx 20 \mu \mathrm{m}$, implying $t_{q}$ to be of the order of $10^{-3} \mathrm{~s}$. From the experiments we infer that the relevant timescale for deformation of the hydrogel and diffusion of the osmolyte molecules is of the order of seconds or tens of seconds, suggesting we have a clear seperation of timescales between the processes of heat conduction and that of mass transport. Similarly, the swollen Aquapearls have $R_{0} \approx 4 \mathrm{~mm}$, implying $t_{q}$ to be in the order of $10^{2} \mathrm{~s}$, while the gel (de)swelling time is many orders of magnitude larger, see Figure 2.2 and 2.3. Therefore, the assumption of a homogeneous temperature throughout the hydrogel, imposed by the surrounding solution, is justified.

To denote the quantity of fluid in an infinitesimal volume $\mathrm{d} \Omega$, we introduce the fluid volume fraction $\phi_{\mathrm{S}}(\boldsymbol{x}, t)$, i.e., the ratio of the volume of fluid in $\mathrm{d} \Omega$, the center of which is located at $\boldsymbol{x}$, to the total volume $\mathrm{d} \Omega$. The volume of fluid in $\mathrm{d} \Omega$ is thus 
given by $\phi_{\mathrm{s}} \mathrm{d} \Omega$. Similarly, we define the polymer network volume fraction $\phi_{\mathrm{n}}$, and the volume fractions satisfy $\phi_{\mathrm{s}}+\phi_{\mathrm{n}}=1$. If we refer to the hydrogel in the following, we refer to the part of space where $\phi_{\mathrm{n}} \neq 0$.

To derive the conservation laws, we consider a volume $\Omega_{t}$ at time $t$. The volume of polymer network $V_{\mathrm{n}, t}$ and the volume of fluid $V_{\mathrm{s}, t}$ in $\Omega_{t}$ can be expressed as

$$
\begin{aligned}
V_{\mathrm{n}, t} & =\int_{\Omega_{t}} \mathrm{~d} \Omega_{t} \phi_{\mathrm{n}}, \\
V_{\mathrm{s}, t} & =\int_{\Omega_{t}} \mathrm{~d} \Omega_{t} \phi_{\mathrm{s}},
\end{aligned}
$$

where $\mathrm{d} \Omega_{t}$ is an infinitesimal volume element and the integral is over the volume $\Omega_{t}$. As noted above, we describe the gel as the superposition of two continua, both filling the entire space. During a short time $\mathrm{d} t$ of the gel deformation, both continua in $\Omega_{t}$ will generally change their shape, location and volume, whilst the volume fractions $\phi_{\mathrm{s}}$ and $\phi_{\mathrm{n}}$ change. Considering, for example, the fluid continuum, we should find the same volume of fluid, $V_{\mathrm{s}, t}$, if we integrate $\phi_{\mathrm{s}}(\boldsymbol{x}, t+\mathrm{d} t)$ over the volume which the fluid continuum occupies after a time $\mathrm{d} t, \Omega_{\mathrm{s}, t+\mathrm{d} t}$, for we consider the fluid mass and specific density to be constant.

To derive the conservation equations, we formalize this statement by introducing the material derivative $\mathrm{d}^{i} / \mathrm{d} t$, which measures the amount of change of a quantity when this quantity is attached to a tiny piece of the $i$-th continuum, a material particle. Mathematically, any point in $\mathbb{R}^{3}$ coincides with a unique material particle of the $i$-th continuum. Using the material derivative, any position vector $\boldsymbol{x}$ changes to a new position vector $\boldsymbol{x}+\mathrm{d} t\left(\mathrm{~d}^{i} \boldsymbol{x} / \mathrm{d} t\right)$. Also, a scalar field $G=G(\boldsymbol{x}, t)$ changes its value when attached to a material particle at position $\boldsymbol{x}$, because both $\boldsymbol{x}$ and $t$ change within a time $\mathrm{d} t$. With $i=\mathrm{n}$ refering to the network continuum and $i=\mathrm{s}$ to the fluid continuum (polymer solution), we state the following relations

$$
\begin{aligned}
\frac{\mathrm{d}^{i} \boldsymbol{x}}{\mathrm{d} t} & =\boldsymbol{v}^{i}, \\
\frac{\mathrm{d}^{i} \mathbf{d} \boldsymbol{x}}{\mathrm{d} t} & =\left(\nabla_{x} \otimes \boldsymbol{v}^{i}\right) \cdot \mathbf{d} \boldsymbol{x}, \\
\frac{\mathrm{d}^{i}\left(\mathrm{~d} \Omega_{t}\right)}{\mathrm{d} t} & =\mathrm{d} \Omega_{t} \nabla_{x} \cdot \boldsymbol{v}^{i}, \\
\frac{\mathrm{d}^{i} G}{\mathrm{~d} t} & =\frac{\partial G}{\partial t}+\boldsymbol{v}^{i} \cdot \nabla_{x} G,
\end{aligned}
$$


where $\boldsymbol{v}^{i}=\boldsymbol{v}^{i}(\boldsymbol{x}, t)$ is the velocity of a material particle at position $\boldsymbol{x}$ on time $t$, $\mathbf{d} \boldsymbol{x}$ is an infinitesimal vector, $\nabla_{x}$ is the gradient operator with respect to position $\boldsymbol{x}$ of a material particle, i.e., the current position, and $\mathrm{d} \Omega_{t}$ is an infinitesimal volume element which is given by the composed product of vectors $\mathbf{d} \boldsymbol{x}_{1}, \mathbf{d} \boldsymbol{x}_{2}$ and $\mathbf{d} \boldsymbol{x}_{3}$, forming a parallelepiped, see Coussy [28] for proofs. Considering an integral over all volume elements $\mathrm{d} \Omega_{t}$ making up the volume $\Omega_{t}$, one can find the rate of change of the volume of the fluid continuum in $\Omega_{t}$ at time $t$ as

$$
\frac{\mathrm{d}^{\mathrm{S}}}{\mathrm{d} t} \int_{\Omega_{t}} \mathrm{~d} \Omega_{t}=\int_{\Omega_{t}}\left(\nabla_{x} \cdot \boldsymbol{v}^{\mathrm{s}}\right) \mathrm{d} \Omega_{t}
$$

In a similar manner, we can obtain the change in the volume of fluid in $\Omega_{t}$, when following the fluid, as

$$
\frac{\mathrm{d}^{\mathrm{s}}}{\mathrm{d} t} \int_{\Omega_{t}} \mathrm{~d} \Omega_{t} \phi_{\mathrm{s}}=\int_{\Omega_{t}} \mathrm{~d} \Omega_{t}\left(\frac{\partial \phi_{\mathrm{s}}}{\partial t}+\nabla_{x} \cdot\left(\phi_{\mathrm{s}} \boldsymbol{v}^{\mathrm{s}}\right)\right) .
$$

The volume of fluid is conserved in time, however, since no fluid is destroyed nor created, and this holds for any volume $\Omega_{t}$. Therefore, the integrand of equation (2.42) should be zero for any volume element $\mathrm{d} \Omega_{t}$, giving rise to the fluid volume conservation equation

$$
\frac{\partial \phi_{\mathrm{s}}}{\partial t}+\nabla_{x} \cdot\left(\phi_{\mathrm{s}} \boldsymbol{v}^{\mathrm{s}}\right)=0
$$

Similarly, by following the network continuum in time we obtain

$$
\frac{\partial \phi_{\mathrm{n}}}{\partial t}+\nabla_{x} \cdot\left(\phi_{\mathrm{n}} \boldsymbol{v}^{\mathrm{n}}\right)=0
$$

Using that $\phi_{\mathrm{s}}+\phi_{\mathrm{n}}=1$, and summing equations (2.43) and (2.44), we immediately find the incompressibility condition for a biphasic porous material

$$
\nabla_{x} \cdot\left(\phi_{\mathrm{s}} \boldsymbol{v}^{\mathrm{s}}+\phi_{\mathrm{n}} \boldsymbol{v}^{\mathrm{n}}\right)=0
$$

which we will invoke in section 2.6 .9 to exploit the symmetry of a spherical gel.

To obtain the osmolyte conservation equation, we consider the number of osmolyte molecules $N_{t}$ in a volume $\Omega_{t}$ as

$$
N_{t}=\int_{\Omega_{t}} \mathrm{~d} \Omega_{t} \phi_{\mathrm{s}} c
$$


where $c$ is the number of osmolyte molecules per unit of fluid volume. Note that $\phi_{\mathrm{S}} c$ gives the number of particles per unit of total volume. To obtain the rate of change of the number of osmolyte molecules in the volume $\Omega_{t}$ we follow the network continuum, because we assume the osmolyte molecules to move advectively with the network. The change of the number of particles in $\Omega_{t}$ must equal the number of particles travelling per second through the boundary $\Omega_{t}$ due to diffusion, giving

$$
\frac{\mathrm{d}^{\mathrm{n}}}{\mathrm{d} t}\left(\int_{\Omega_{t}} \mathrm{~d} \Omega_{t} \phi_{\mathrm{s}} c\right)+\int_{\partial \Omega_{t}} \mathrm{~d} S_{t} \hat{n} \cdot \boldsymbol{m}_{\mathrm{dif}}=0,
$$

where $\partial \Omega_{t}$ is the boundary of $\Omega_{t}, \mathrm{~d} S_{t}$ is an infinitesimal area on $\partial \Omega_{t}, \hat{n}$ is the outward pointing unit vector normal to $\partial \Omega_{t}$ at the position of $\mathrm{d} S_{t}$, and $\boldsymbol{m}_{\mathrm{dif}}$ is the flux of polymer particles through the boundary due to diffusion. The particle flux through the boundary is purely diffusive because we assume the osmolyte molecules to move advectively along with the network. We assume the osmolyte molecules to obey Fick's law, so

$$
\boldsymbol{m}_{\mathrm{dif}}=-D_{\mathrm{osm}} \nabla_{x} c,
$$

where $D_{\text {osm }}$ is the osmolyte diffusion constant inside the polymer network of the gel. We expect $D_{\text {osm }}$ to be significantly lower than the diffusion constant in pure water due to hindrance of the hydrogel network. Generally, $D_{\text {osm }}$ is taken to be a function of the fluid volume fraction $\phi_{\mathrm{s}}$, the radius of gyration of the osmolyte molecules $R_{\mathrm{g}}$ dissolved polymer radius $r_{s o l}$ and the permeability of the hydrogel $k$. With the help of Gauss' divergence theorem we obtain from equation (2.47)

$$
\frac{\partial \phi_{\mathrm{s}} c}{\partial t}+\nabla_{x} \cdot\left(\phi_{\mathrm{s}} c \boldsymbol{v}^{\mathrm{n}}\right)+\nabla_{x} \cdot \boldsymbol{m}_{\mathrm{dif}}=0,
$$

the convection-diffusion equation for relatively large osmolyte molecules dissolved in fluid penetrating a porous medium.

The conservation equations, equation (2.43), (2.44) and (2.49), are in their Eulerian form, referring to the position, $\boldsymbol{x}$, of a volume element at time $t$. To solve them one needs appropiate boundary conditions for the (possibly) moving boundary, besides additional equations of motion for the bulk. Instead, the Lagrangian form of these equations makes reference to the labels given to the material particles of the network continuum and time $t$. The label given to the material particles is typically their position at $t=0$. Therefore, the boundary conditions are more easily specified in a Lagrangian framework as they are continuously imposed at the same material particle 
of the network continuum. Furthermore, when working with Lagrangian quantities the material derivative $\mathrm{d}^{\mathrm{n}} / \mathrm{d} t$ simplifies to $\partial / \partial t$. In the following we will cast all three conservation equations in their Lagrangian form.

\section{Lagrangian framework}

To cast the conservation equations (2.43), (2.44) and (2.49) in their Lagrangian form we first consider equation (2.44) and rewrite it to

$$
\frac{\mathrm{d}^{\mathrm{n}}}{\mathrm{d} t}\left(\phi_{\mathrm{n}} \mathrm{d} \Omega_{t}\right)=0
$$

where we used equation (2.40) and (2.39). This equation implies that if we consider an infinitesimal volume element $\mathrm{d} \Omega_{t}$ at any time $t$ and position $\boldsymbol{x}$, and follow in time the network continuum in this volume element, then the volume of network in this piece of network continuum does not change. Therefore, we have

$$
\phi_{\mathrm{n}} \mathrm{d} \Omega_{t}=\phi_{\mathrm{n}, 0} \mathrm{~d} \Omega_{0},
$$

with $\phi_{\mathrm{n}, 0}$ the volume fraction of network in the network continuum volume element at $t=0$, and $\mathrm{d} \Omega_{0}$ its volume at $t=0$.

To relate the volume of an infinitesimal volume element of the network continuum at time $t$ to its original volume at time $t=0$, and because the stress-strain relation we invoke in the following sections is expressible in it, we introduce the deformation gradient $\boldsymbol{F}$, which relates any infinitesimal vector attached to the network continuum, $\mathbf{d} \boldsymbol{x}$, to this same vector at $t=0, \mathbf{d} \boldsymbol{X}$, through

$$
\begin{aligned}
\mathbf{d} \boldsymbol{x} & =\boldsymbol{F} \cdot \mathbf{d} \boldsymbol{X} \\
d x_{i} & =\frac{\partial x_{i}}{\partial X_{j}} d X_{j} .
\end{aligned}
$$

So $(\boldsymbol{F})_{i j}=\frac{\partial x_{i}}{\partial X_{j}}$, and $\left(\boldsymbol{F}^{-1}\right)_{i j}=\frac{\partial X_{i}}{\partial x_{j}}$. Using equation (2.52) we can write for the infinitesimal volume $\mathrm{d} \Omega_{t}$ at time $t$

$$
\begin{aligned}
\mathrm{d} \Omega_{t} & \equiv \mathbf{d} \boldsymbol{x}_{1} \cdot\left(\mathbf{d} \boldsymbol{x}_{2} \times \mathbf{d} \boldsymbol{x}_{3}\right), \\
& =\operatorname{det}(\boldsymbol{F}) \mathbf{d} \boldsymbol{X}_{1} \cdot\left(\mathbf{d} \boldsymbol{X}_{2} \times \mathbf{d} \boldsymbol{X}_{3}\right), \\
& =\operatorname{det}(\boldsymbol{F}) \mathrm{d} \Omega_{0}, \\
& =J \mathrm{~d} \Omega_{0},
\end{aligned}
$$


where we defined $J \equiv \operatorname{det}(\boldsymbol{F})$. Using equation (2.51) with equation (2.57) we obtain

$$
\phi_{\mathrm{n}} J=\phi_{\mathrm{n}, 0},
$$

which relates the volume fraction of network at time $t$ to the volume fraction at of network at $t=0$. In a similar way we define the ratio of the volume of fluid in a volume $\mathrm{d} \Omega_{t}$ to the volume which the network continuum in $\mathrm{d} \Omega_{t}$ had at $t=0$ as

$$
\Phi_{\mathrm{S}} \equiv J \phi_{\mathrm{s}}
$$

Accordingly, we define the volume fraction of network with respect to the network continuum configuration at $t=0$ as

$$
\Phi_{\mathrm{n}} \equiv J \phi_{\mathrm{n}}
$$

and from equation (2.51) it follows immediately that $\Phi_{\mathrm{n}}=\phi_{\mathrm{n}, 0}$ and since this holds for all times we have $\Phi_{\mathrm{n}}=\Phi_{\mathrm{n}, 0}$, which is a restatement that the volume of network is conserved if one follows a volume element of the network continuum.

Using $\Phi_{\mathrm{S}}$ we can cast equation (2.43) in its Lagrangian form in the following way. First, we define $\boldsymbol{w}$, the fluid flux which passes through an infinitesimal area attached to the network continuum as

$$
\boldsymbol{w} \equiv \phi_{\mathrm{s}}\left(\boldsymbol{v}^{\mathrm{s}}-\boldsymbol{v}^{\mathrm{n}}\right)
$$

such that the volume passing through a surface $\mathrm{d} S_{t}$ is equal to $\hat{n} \cdot \boldsymbol{w} \mathrm{d} S_{t}$, with $\hat{n}$ the normal vector to the surface. Using this definition we find for equation (2.43)

$$
\begin{aligned}
& \frac{\partial \phi_{\mathrm{s}}}{\partial t}+\nabla_{x} \cdot\left(\phi_{\mathrm{s}} \boldsymbol{v}^{\mathrm{n}}\right)+\nabla_{x} \cdot \boldsymbol{w}=0 \\
& \frac{\mathrm{d}^{\mathrm{n}} \phi_{\mathrm{s}}}{\mathrm{d} t}+\phi_{\mathrm{s}} \nabla_{x} \cdot \boldsymbol{v}^{\mathrm{n}}+\nabla_{x} \cdot \boldsymbol{w}=0
\end{aligned}
$$

With equation (2.59) we have $\phi_{\mathrm{s}} \mathrm{d} \Omega_{t}=\Phi_{\mathrm{s}} \mathrm{d} \Omega_{0}$, so we write

$$
\frac{\mathrm{d}^{\mathrm{n}} \phi_{\mathrm{s}}}{\mathrm{d} t}=\frac{\mathrm{d} \Omega_{0}}{\mathrm{~d} \Omega_{t}}\left(\frac{\mathrm{d}^{\mathrm{n}} \Phi_{\mathrm{s}}}{\mathrm{d} t}-\frac{\Phi_{\mathrm{s}}}{\mathrm{d} \Omega_{t}} \frac{\mathrm{d}^{\mathrm{n}} \mathrm{d} \Omega_{t}}{\mathrm{~d} t}\right)=\frac{\mathrm{d} \Omega_{0}}{\mathrm{~d} \Omega_{t}} \frac{\mathrm{d}^{\mathrm{n}} \Phi_{\mathrm{s}}}{\mathrm{d} t}-\phi_{\mathrm{s}} \nabla_{x} \cdot \boldsymbol{v}^{\mathrm{n}},
$$

such that we obtain for equation (2.63)

$$
\mathrm{d} \Omega_{0} \frac{\mathrm{d}^{\mathrm{n}} \Phi_{\mathrm{s}}}{\mathrm{d} t}+\mathrm{d} \Omega_{t} \nabla_{x} \cdot \boldsymbol{w}=0
$$


As $\nabla_{x}$ is the gradient operator in space we would like to rewrite it in terms of the positions $\boldsymbol{X}$ of the network continuum at $t=0$, such that we can use $\boldsymbol{X}$ as a label for the material particles of the network and express the fluid conservation equation as a function of the independent variables $\boldsymbol{X}$ and $t$. To do so we consider again the flux through a surface attached to the network continuum which has an area $\mathrm{d} S_{t}$ and normal vector $\hat{n}$ at time $t$, and express this flux as a vector $\boldsymbol{W}$ with respect to the state of the netwerk continuum at $t=0$, which satisfies

$$
\boldsymbol{w} \cdot \hat{n} \mathrm{~d} S_{t}=\boldsymbol{W} \cdot \hat{N} \mathrm{~d} S_{0},
$$

where $\mathrm{d} S_{0}$ is the area of and $\hat{N}$ is the normal vector to the material surface under consideration at $t=0$. Using Gauss' theorem we obtain from equation (2.66)

$$
\mathrm{d} \Omega_{t} \nabla_{x} \cdot \boldsymbol{w}=\mathrm{d} \Omega_{0} \nabla_{X} \cdot \boldsymbol{W},
$$

and with Nanson's formula it follows from equation (2.66) that $\boldsymbol{W} \equiv J \boldsymbol{F}^{-1} \boldsymbol{w}$, with $\boldsymbol{F}^{-1}$ the inverse deformation gradient. With the help of equation (2.67) we obtain the Lagrangian form of the fluid conservation equation from equation (2.65) as

$$
\frac{\partial \Phi_{\mathrm{s}}}{\partial t}+\nabla_{X} \cdot \boldsymbol{W}=0,
$$

where we consider $\Phi_{\mathrm{s}}=\Phi_{\mathrm{S}}(\boldsymbol{X}, t)$ as a function of position of the material particles of the network continuum in the reference configuration $\boldsymbol{X}$ at $t=0$, the material particle time derivative following the network continuum reduces to a partial time derivative, and $\boldsymbol{W}=J \boldsymbol{F}^{-1} \phi_{\mathrm{s}}\left(\boldsymbol{V}^{\mathrm{s}}-\boldsymbol{V}^{\mathrm{n}}\right)$ with $\boldsymbol{V}^{\mathrm{s}}=\boldsymbol{V}^{\mathrm{s}}(\boldsymbol{X}, t)$ the speed of a material particle of the fluid continuum which is at the same position at time $t$ as the material particle of the network continuum which was located at $\boldsymbol{X}$ at $t=0$. Furthermore, $\boldsymbol{V}^{\mathrm{n}}=\boldsymbol{V}^{\mathrm{n}}(\boldsymbol{X}, t)$ is the speed of a material particle of the network continuum originally located at position $\boldsymbol{X}$ at time $t$. These speeds obey $\boldsymbol{V}^{i}(\boldsymbol{X}, t)=$ $\boldsymbol{v}^{i}(\boldsymbol{x}, t)$, where $\boldsymbol{v}^{i}(\boldsymbol{x}, t)$ is the speed of the material particle of the $i$-th continuum located at position $\boldsymbol{x}$ at time $t$.

To write the osmolyte conservation equation, equation (2.49), in Lagrangian form, we first invoke equation (2.44) and (2.40) to obtain

$$
\phi_{\mathrm{s}} \frac{\mathrm{d}^{\mathrm{n}} c}{\mathrm{~d} t}+c \nabla_{x} \cdot \boldsymbol{v}^{\mathrm{n}}+\nabla_{x} \cdot \boldsymbol{m}_{\text {dif }}=0,
$$

where $\boldsymbol{m}_{\text {dif }}=-D_{\mathrm{osm}} \nabla_{x} c$. Next, multiplying equation (2.69) with the volume of an infinitesimal volume element $\mathrm{d} \Omega_{t}$ located at the position where we consider the 
osmolyte conservation, we can write

$$
\Phi_{\mathrm{s}} \frac{\mathrm{d}^{\mathrm{n}} c}{\mathrm{~d} t}+c \nabla_{X} \cdot J \boldsymbol{F}^{-1} \boldsymbol{V}^{\mathrm{n}}+\nabla_{X} \cdot \boldsymbol{M}_{\mathrm{dif}}=0
$$

where we used $\mathrm{d} \Omega_{t} \phi_{\mathrm{s}}=\mathrm{d} \Omega_{0} \Phi_{\mathrm{s}}$, equation (2.67), defined $\boldsymbol{M} \equiv J \boldsymbol{F}^{-1} \boldsymbol{m}_{\mathrm{dif}}$ and $\nabla_{X}$ denotes a gradient operator with respect to the coordinates of the reference configuration. Taking all quantities in equation (2.70) as a function of $\boldsymbol{X}$ and $t$ we obtain

$$
\Phi_{\mathrm{s}} \frac{\partial c}{\partial t}+c \nabla_{X} \cdot J \boldsymbol{F}^{-1} \boldsymbol{V}^{\mathrm{n}}+\nabla_{X} \cdot \boldsymbol{M}_{\mathrm{dif}}=0
$$

with $\boldsymbol{M}_{\text {dif }} \equiv-J \boldsymbol{F}^{-1} \boldsymbol{F}^{-T} D_{\mathrm{osm}} \nabla_{X} c$ and $\Phi_{\mathrm{s}}=J \phi_{\mathrm{s}}$. Equation (2.71) expresses the change in time of the number of osmolyte molecules per unit of fluid volume in an infinitesimal volume element of the network continuum. The second term quantifies the change in the concentration of osmolyte molecules due to compression or expansion of the network. To understand this, recall that the osmolyte molecules move advectively with the network due to their size. The third accounts for the diffusional flux of osmolyte in or out of the material particle.

\subsubsection{Force balance}

The force balance for a material volume $\mathrm{d} \Omega_{t}$ can be derived using the hypothesis of local forces, the linear and angular momentum balance of the fluid and network continuum combined, Newtons third law, and the tetrahedon lemma, see Coussy [28] for more details. The force balance for all matter inside an infinitesimal volume element $\mathrm{d} \Omega_{t}$ can be expressed for a biphasic material as

$$
\nabla_{x} \cdot \boldsymbol{\sigma}+\boldsymbol{f}^{\mathrm{n}}+\boldsymbol{f}^{\mathrm{s}}=\phi_{\mathrm{n}} \rho_{\mathrm{n}} \boldsymbol{a}^{\mathrm{n}}+\phi_{\mathrm{s}} \rho_{\mathrm{s}} \boldsymbol{a}^{\mathrm{s}},
$$

where $\boldsymbol{\sigma}$ is the Cauchy stress tensor which gives the force $\boldsymbol{T}$ on a material surface $\mathrm{d} S_{t}$ of $\mathrm{d} \Omega_{t}$ as

$$
\boldsymbol{T}=\boldsymbol{\sigma} \hat{n},
$$

with $\hat{n}$ the unit vector normal to $\mathrm{d} S_{t}$. Furthermore, $\boldsymbol{f}^{i}$ is the external force per unit of volume acting on the volume element of the $i$-th continuum in $\mathrm{d} \Omega_{t}$, with $i=\mathrm{s}$ for the fluid continuum and $i=\mathrm{n}$ for the network continuum. The specific density of the $i$-th continuum is $\rho_{i}$ and $\phi_{i}$ is its volume fraction. Recall that we describe the gel as a superposition of a fluid and network continuum, both filling the entire space. 
The force of gravity, for example, can be written as $\boldsymbol{f}^{i}=\phi_{i} \rho_{i} \boldsymbol{g}$, where $\boldsymbol{g}=g \hat{z}$ and $g$ is the gravitational acceleration constant. In the dynamics of spherical gels, immersed in liquid, gravity plays no role for the hydrostatic pressure of the outside liquid balances that in the hydrogel, so we can set $g=0$. The accelerations $\boldsymbol{a}^{i}$, defined as $\boldsymbol{a}^{i}=\mathrm{d}^{i} \boldsymbol{v}^{i} / \mathrm{d} t$, are generally negligible because by dimensional scaling, as will be done for the equations of motion in the next sections, one finds that the acceleration terms scale as the ratio of the typical kinetic energy per unit volume and the typical size of the Terzaghi effective stress of the polymer network, $\phi_{i} \rho_{i}\left(a / \tau_{\mathrm{n}}\right)^{2} / K$, where $R_{0}$ is the radius of the gel, $\tau_{\mathrm{n}}$ is the typical time for fluid to flow through the network, i.e., the typical time needed for the network to deform and $K$ is the bulk modulus of the hydrogel network. Given these considerations, we obtain as force balance equation

$$
\nabla_{x} \cdot \boldsymbol{\sigma}=0
$$

expressing a state of mechanical equilibrium, where $\sigma$ is the Cauchy stress tensor accounting for all forces per unit area which all matter outside of a volume element $\mathrm{d} \Omega_{t}$ exert on the surfaces of this volume element.

\section{Stress Tensor}

To express $\boldsymbol{\sigma}$ in terms of our dependent variables we use the concept of Terzaghi's effective stress: the difference between the total stress and the total fluid pressure $P$ [27]. Microscopically, the fluid permeates the polymer network, causing the polymer network to inherit the pressure of the fluid. Therefore, one can write the total stress as the sum of fluid pressure and a surplus, the Terzaghi effective stress due to the hydrogel network $\sigma^{\prime}$, giving

$$
\boldsymbol{\sigma}=\boldsymbol{\sigma}^{\prime}-P \mathbb{1}
$$

Next, imagine an infinitesimal volume $\mathrm{d} \Omega_{t}$ inside the gel with a number of osmolyte molecules per unit of fluid volume $c$. Using one of the basic assumptions of nonequilibrium thermodynamics [28], the local equilibrium assumption, we suppose it is reasonable to define an osmotic pressure of the osmolyte molecules in $d \Omega_{t}, \Pi_{\text {osm }}$. Subsequently, one can speak of the osmotic stress on the surface surrounding the volume element $\mathrm{d} \Omega_{t}$ in the same way one can speak of the osmotic stress reducing the size of a hydrogel when its network effectively acts as a semi-permeable membrane 
to the surrounding solution. Given these considerations, we write the total pressure that the network inherits, as

$$
P=p+\Pi_{\mathrm{osm}},
$$

with $p$ the pneumatic pressure and $\Pi_{\mathrm{osm}}$ the osmotic pressure of the osmolyte molecules. In the following we assume a virial expansion for the osmotic pressure: $\Pi_{\mathrm{osm}}=\Pi_{\mathrm{osm}}(c)$, and for simplicity we neglect contributions to the pressure stemming from hydrogel-osmolyte interactions, i.e., changes in solvent quality. Furthermore, we use Hencky elasticity as a constitutive relation relating $\sigma^{\prime}$ to the deformation gradient of the network continuum $\boldsymbol{F}$ as

$$
J \boldsymbol{\sigma}^{\prime}=\Lambda \operatorname{tr}(\boldsymbol{H}) \mathbb{1}+(M-\Lambda) \boldsymbol{H},
$$

with $\boldsymbol{H}=\frac{1}{2} \ln \boldsymbol{F} \boldsymbol{F}^{T}, \Lambda=K-2 G / 3$ Lamé's first constant, $M=K+4 G / 3$ the longitudinal modulus, $K$ the bulk modulus of the hydrogel network and $G$ the shear modulus of the hydrogel network. We choose Hencky elasticity as a phenomenological constitutive relation because it reduces to linear elasticity for small strains but measures the natural (logarithmic) strain in three dimensions. Furthermore, because a covalently bonded hydrogel network typically has a Poisson ratio close to 0.5 , therefore behaving much like rubber, we deem Hencky elasticity with constant material parameters a suitable contitutive relation.

\section{Lagrangian formulation}

It can be shown [28] that $\boldsymbol{\sigma}: \boldsymbol{d}^{\mathrm{n}} \mathrm{d} \Omega_{t}$ is the work done in a volume $\mathrm{d} \Omega_{t}$ due to strain of the network continuum per unit of time, where $\boldsymbol{d}^{\mathrm{n}} \equiv \frac{1}{2}\left(\nabla_{x} \otimes \boldsymbol{V}^{\mathrm{n}}+\left(\nabla_{x} \otimes \boldsymbol{V}^{\mathrm{n}}\right)^{T}\right)$ is the Eulerian strain rate tensor, $\boldsymbol{\sigma}$ is the Cauchy stress tensor and $\boldsymbol{\sigma}: \boldsymbol{d}^{\mathrm{n}}=\sigma_{i j} d_{i j}^{\mathrm{n}}$. The Lagrangian counterpart of the Eulerian strain rate tensor is the time derivative of the Green-Lagrange strain tensor, $d \boldsymbol{\Delta} / \mathrm{d} t$. To express the strain power in Lagrangian quantities we define the Piola-Kirchhoff stress tensor $\boldsymbol{\pi}$ to satisfy

$$
\boldsymbol{\sigma}: \boldsymbol{d}^{\mathrm{n}} \mathrm{d} \Omega_{t}=\boldsymbol{\pi}: \frac{d \boldsymbol{\Delta}}{\mathrm{d} t} \mathrm{~d} \Omega_{0},
$$

where $\mathrm{d} \Omega_{0}$ is the volume, at $t=0$, of the volume element of the network continuum present in $\mathrm{d} \Omega_{t}$. Using that $\boldsymbol{d}^{\mathrm{n}}=\boldsymbol{F}^{-T} \frac{d \boldsymbol{\Delta}}{\mathrm{d} t} \boldsymbol{F}^{-1}$, and $\mathrm{d} \Omega_{t}=J \mathrm{~d} \Omega_{0}$, we obtain from equation (2.78)

$$
\boldsymbol{\pi}=J \boldsymbol{F}^{-1} \boldsymbol{\sigma} \boldsymbol{F}^{-T}
$$


Multiplying equation (2.74) with $\mathrm{d} \Omega_{t}$, and using equations (2.67) and (2.79), we find

$$
\nabla_{X} \cdot(\boldsymbol{F} \boldsymbol{\pi})=0
$$

enabling us to write the force balance equation as a function of time $t$ and the position of the volume elements of the network continuum at $t=0$, and material particle label $\boldsymbol{X}$.

\subsubsection{Spherical symmetry}

As we consider a spherical gel we assume the deformation of the gel and the flow of fluid to occur radially and to depend only on the radial coordinate $r$, so $\boldsymbol{v}^{i}=v^{i}(r, t) \hat{r}$. Also, the volume fraction of the fluid, $\phi_{\mathrm{s}}$, and the volume fraction of polymer network, $\phi_{\mathrm{n}}$, are assumed to depend only on $r$. Next, we define the volume averaged center of mass speed, $\boldsymbol{v}_{\mathrm{cm}}$, as $\boldsymbol{v}_{\mathrm{cm}} \equiv \phi_{\mathrm{s}} \boldsymbol{v}^{\mathrm{s}}+\phi_{\mathrm{n}} \boldsymbol{v}^{\mathrm{n}}=\left(\phi_{\mathrm{s}} v^{\mathrm{s}}+\phi_{\mathrm{n}} v^{\mathrm{n}}\right) \hat{r}=v_{\mathrm{cm}}(r, t) \hat{r}$. Using the incompressibility condition, equation (2.45), and Gauss' theorem we can write

$$
0=\int_{\Omega_{t}} \mathrm{~d} \Omega_{t} \nabla_{x} \cdot \boldsymbol{v}_{\mathrm{cm}}=\int_{\partial \Omega_{t}} \mathrm{~d} S_{t} \hat{n} \cdot \boldsymbol{v}_{\mathrm{cm}},
$$

where $\Omega_{t}$ is an arbitrary volume in the gel, $\partial \Omega_{t}$ is its boundary, and $\hat{n}$ is the outward pointing normal vector to $\partial \Omega_{t}$ at the position of an infinitesimal area element $\mathrm{d} S_{t}$ on the boundary. Taking $\Omega_{t}$ to be a sphere of radius $r$, it follows that

$$
0=4 \pi r^{2} v_{\mathrm{cm}}
$$

implying for any $r>0$

$$
v^{\mathrm{s}}=-\frac{\phi^{\mathrm{n}}}{\phi^{\mathrm{s}}} v^{\mathrm{n}} .
$$

Using equation (2.83), we find the flux of fluid through an infinitesimal surface attached to the network continuum to be

$$
\boldsymbol{w}=\phi_{\mathrm{s}}\left(\boldsymbol{v}^{\mathrm{s}}-\boldsymbol{v}^{\mathrm{n}}\right)=-\boldsymbol{v}^{\mathrm{n}}
$$

In the next section we show how the volume conservation equations and the force balance constitute a set of equations of motion for the deformation of the network continuum and the concentration of osmolyte molecules. Equation (2.84) and Darcy's law will play an essential role in this derivation. 


\subsubsection{Equations of motion}

In the previous sections we found Lagrangian equations of motion governing the transport of the osmolyte molecules, the fluid and the hydrogel network as a function of time $t$ and the position $\boldsymbol{X}$ of the volume elements of the network continuum in the reference configuration at $t=0$. The equations of motion are as follows

$$
\begin{aligned}
& 0=\frac{\partial \Phi_{\mathrm{s}}}{\partial t}+\nabla_{X} \cdot \boldsymbol{W}, \\
& 0=\Phi_{\mathrm{s}} \frac{\partial c}{\partial t}+c \nabla_{X} \cdot J \boldsymbol{F}^{-1} \boldsymbol{V}^{\mathrm{n}}+\nabla_{X} \cdot \boldsymbol{M}_{\mathrm{dif}}, \\
& 0=\nabla_{X} \cdot(\boldsymbol{F} \boldsymbol{\pi}),
\end{aligned}
$$

where $\Phi_{\mathrm{s}}$ is the ratio of the volume of fluid in an infinitesimal volume element $\mathrm{d} \Omega_{t}$ to the volume of this element at $t=0, \boldsymbol{W}=J \boldsymbol{F}^{-1} \phi_{\mathrm{s}}\left(\boldsymbol{V}^{\mathrm{s}}-\boldsymbol{V}^{\mathrm{n}}\right)$ is the flux vector of fluid through a surface attached to the network continuum, $\boldsymbol{V}^{i}$ is the velocity vector of network $(i=\mathrm{n})$ or fluid $(i=\mathrm{s})$ continuum, $c$ is the number of osmolyte molecules per unit of fluid volume, $\boldsymbol{M}=-J \boldsymbol{F}^{-1} \boldsymbol{F}^{-T} D_{\mathrm{osm}} \nabla_{X} c$ is the diffusive flux of osmolyte molecules, $\boldsymbol{F}$ is the deformation gradient, $J=\operatorname{det}(\boldsymbol{F})$, and $\boldsymbol{F} \boldsymbol{\pi}=J \boldsymbol{\sigma} \boldsymbol{F}^{-T}$ with $\boldsymbol{\sigma}$ the Cauchy stress tensor. The five unknowns are $\Phi_{\mathrm{s}}, \boldsymbol{V}^{\mathrm{s}}, \boldsymbol{V}^{\mathrm{n}}, c$ and $\boldsymbol{\sigma}$. Nonetheless, we can find a closed set of equations from the following considerations.

First, we introduce the deformation vector field $\boldsymbol{U}=\boldsymbol{U}(\boldsymbol{X}, t)$ satisfying $\boldsymbol{x}(\boldsymbol{X}, t)=$ $\boldsymbol{X}+\boldsymbol{U}$, with $\boldsymbol{X}$ the position of a piece of the hydrogel network at $t=0$ and $\boldsymbol{x}$ the position of this piece at time $t$. Rewriting the left hand side of equation (2.37) as $\mathrm{d}^{\mathrm{n}} \boldsymbol{x} / \mathrm{d} t=\partial \boldsymbol{x}(\boldsymbol{X}, t) / \partial t=\partial \boldsymbol{U} / \partial t$, it follows immediately that

$$
\frac{\partial \boldsymbol{U}}{\partial t}=\boldsymbol{V}^{\mathrm{n}}
$$

Also, in the case of a spherically symmetric hydrogel we have

$$
\boldsymbol{V}^{\mathrm{s}}=-\frac{\phi_{\mathrm{n}}}{\phi_{\mathrm{s}}} \boldsymbol{V}^{\mathrm{n}}
$$

and $\boldsymbol{V}^{i}=V^{i}(R, t) \hat{R}$, as shown in section 2.6.9, causing $\boldsymbol{V}^{\mathrm{s}}$ to be expressible in terms of $\boldsymbol{U}$. Furthermore, we can write $\boldsymbol{F}$ in spherical coordinates as $\boldsymbol{F}=$ $\operatorname{diag}\left(1+\frac{\partial U}{\partial R}, 1+\frac{U}{R}, 1+\frac{U}{R}\right) \equiv \operatorname{diag}\left(\lambda_{r}, \lambda_{\theta}, \lambda_{\theta}\right)$, with $R$ the original radial position at $t=0$ of hydrogel network in an infinitesimal volume element located at $\boldsymbol{x}$ 
at some time $t, R \in] 0, a]$, and $R_{0}$ being the radius of the hydrogel at $t=0$. With equation (2.59) and (2.60) it follows that $J=J\left(\phi_{\mathrm{s}}+\phi_{\mathrm{n}}\right)=\Phi_{\mathrm{s}}+\Phi_{\mathrm{n}}$, and since $\Phi_{\mathrm{n}}=\Phi_{\mathrm{n}, 0}=1-\Phi_{\mathrm{s}, 0}$, see section 2.6.7, we have $J=1+\Phi_{\mathrm{s}}-\Phi_{\mathrm{s}, 0}$. Finally, because $J=\operatorname{det}(\boldsymbol{F})=\lambda_{r} \lambda_{\theta}^{2}, \Phi_{\mathrm{s}}$ can be expressed in terms of $U(R, t)$.

Using the above considerations, $\boldsymbol{V}^{\mathrm{s}}, \boldsymbol{V}^{\mathrm{n}}$ and $\Phi_{\mathrm{s}}$ can be expressed in terms of $U(R, t)$ and we are left with three dependent variables and three equations. However, using equation (2.89) we have $\boldsymbol{W}=-J \boldsymbol{F}^{-1} \boldsymbol{V}^{\mathrm{n}}$ and one finds that equation (2.85) is trivially satisfied. Because in deriving equation (2.89) we used equation (2.45) which was found by combining the fluid and network conservation equations, equation (2.85) is trivially satisfied.

To find a closed set of equations we use the derivation of the Cauchy stress tensor from section 2.6.8 and write it as

$$
\boldsymbol{\sigma}=\boldsymbol{\sigma}^{\prime}-\left(p+\Pi_{\mathrm{osm}}\right) \mathbb{1}
$$

where $\sigma^{\prime}$ is the Terzaghi effective stress tensor given by equation (2.77) and is expressed in $U(R, t)$ through $\boldsymbol{F}$. Next, we invoke Darcy's law, an empirical law governing the flow through porous media, which allows us to express the pressure in terms of the flux vector of fluid as

$$
\boldsymbol{w}=-\frac{k}{\eta} \nabla_{x} p,
$$

where $k$ is the permeability of the network, $\eta$ is the dynamic viscosity of the fluid in which the osmolyte molecules are dissolved, i.e., the dynamic viscosity of water for our experiments, and $\boldsymbol{w}=\phi_{\mathrm{s}}\left(\boldsymbol{v}^{\mathrm{s}}-\boldsymbol{v}^{\mathrm{n}}\right)$. The permeability is generally a function of the volume fraction of network, the concentration of osmolyte molecules and the diffusion constant of the osmolyte. In Lagrangian coordinates, equation (2.91) is written as

$$
\boldsymbol{F}^{T} \boldsymbol{V}^{\mathrm{n}}=\frac{k}{\eta} \nabla_{X} p
$$

upon using equation (2.89). Using Darcy's law, we find two equations of motion for $U(R, t)$ and $c(R, t)$ as

$$
\begin{array}{r}
\frac{\eta}{k} \frac{\partial U}{\partial t}-\frac{1}{\lambda_{r}} \frac{\partial \sigma_{r r}^{\prime}}{\partial R}-\frac{2}{R} \frac{\left(\sigma_{r r}^{\prime}-\sigma_{\theta \theta}^{\prime}\right)}{\lambda_{\theta}}+\frac{\partial \Pi_{\mathrm{osm}} / \partial c}{\lambda_{r}} \frac{\partial c}{\partial R}=0, \\
\Phi_{\mathrm{s}} \frac{\partial c}{\partial t}-\frac{1}{R^{2}} \frac{\partial}{\partial R}\left(\lambda_{\theta} R\right)^{2} \frac{D_{\mathrm{osm}}}{\lambda_{r}} \frac{\partial c}{\partial R}+c \frac{1}{R^{2}} \frac{\partial}{\partial R}\left(\lambda_{\theta} R\right)^{2} \frac{\partial U}{\partial t}=0,
\end{array}
$$


which is essentially a set of coupled diffusion-like equations for $U(R, t)$ and $c(R, t)$. Each equation has a source term generated by the other dependent variable. To derive these equations we used

$$
\nabla_{X} \cdot \boldsymbol{A}=\left(\frac{\partial A_{r r}}{\partial R}+\frac{2}{R}\left[A_{r r}-A_{\theta \theta}\right]\right) \hat{R}
$$

for a matrix $\boldsymbol{A}=\operatorname{diag}\left(A_{r r}, A_{\theta \theta}, A_{\theta \theta}\right)$ which depends only on the radial coordinate $R$. Also, we observe the identity $\frac{\partial \lambda_{\theta}^{2}}{\partial R}+\frac{2}{R} \lambda_{\theta}\left(\lambda_{\theta}-\lambda_{r}\right)=0$. The components of the Terzaghi effective stress tensor $\boldsymbol{\sigma}^{\prime}=\operatorname{diag}\left(\sigma_{r r}^{\prime}, \sigma_{\theta \theta}^{\prime}, \sigma_{\theta \theta}^{\prime}\right)$ are given by

$$
\begin{aligned}
\sigma_{r r}^{\prime} & =\frac{M \ln \lambda_{r}+2 \Lambda \ln \lambda_{\theta}}{\lambda_{r} \lambda_{\theta}^{2}}, \\
\sigma_{\theta \theta}^{\prime} & =\frac{\Lambda \ln \lambda_{r}+(M+\Lambda) \ln \lambda_{\theta}}{\lambda_{r} \lambda_{\theta}^{2}},
\end{aligned}
$$

where $M \equiv K+4 G / 3$ is called the longitudinal modulus and $\Lambda=K-2 G / 3$ is Lamé's first parameter.

Finally, in order to solve these equations appropiate boundary and initial conditions for $c(R, t)$ and $U(R, t)$ are required. For notational simplicity, we consider in the rest of this Appendix the (hypothetical) case where the partition coefficient equals unity, $Q=1$. Generalization to the more general case with $Q \neq 1$, which we considered in section 2.4.1, is straightforward. Force balance at the boundary of the hydrogel demands the traction force exerted on the hydrogel by the surrounding solution to be balanced by the stress in the hydrogel: $\boldsymbol{f}_{\text {ext }}=\hat{r} \boldsymbol{\sigma}$. The solution at the boundary of the hydrogel is assumed to have a mechanical pressure $p_{0}$ and an osmotic pressure $\Pi_{0}$. We neglect the pressure drop in the running solution over the distance of the hydrogel in the microgel experiments. Also, the concentration of osmolyte molecules in the solution at the boundary is taken to be a constant $c_{0}$ because the hydrogel is immersed in a running/mixed solution, causing osmolyte molecules to be constantly replenished. Experimentally, we verified that lowering the flow velocity in the microgel experiments does not alter the evolution of the hydrogel in a polymer solution. Given these considerations we obtain as a force balance on the hydrogel surface in the radial direction

$$
\sigma_{r r}^{\prime}(a, t)=-\left(\Pi_{0}-\Pi_{\mathrm{osm}}(c(a, t))\right)-\left(p_{0}-p(a, t)\right),
$$

where $R_{0}$ is the radius of the hydrogel at $t=0$ and $\sigma_{r r}^{\prime}(a, t)$ is given by equation (2.96). As we assume the partition coefficient to be unity, the hydrogel network is 
effectively regarded as a phantom network, implying $c(a, t)=c_{0}$ for $t>0$. Also, because the hydrogel boundary is permeable for water we assume the pressure of water to be continuous: $p(a, t)=p_{0}$. These conditions provide a condition at $R=a$ for both $U(R, t)$ and $c(R, t)$ for $t>0$. At the center of the hydrogel $U(R, t)$ should go to zero, for a point can not deform into a spherical surface: $U(0, t)=0$. We expect the concentration of osmolyte molecules $c(R, t)$ to be a continuously differentiable function inside the hydrogel when moving on a line from one edge of the hydrogel through the center to the other edge, therefore $\partial c / \partial R=0$ at $R=0$. The osmolyte molecule concentration inside the hydrogel is zero before the hydrogel is put into the polymer solution, implying $c(R, 0)=0$, and the hydrogel is initially undeformed, giving $U(R, 0)=0$. With these four boundary and two initial conditions equation (2.93) and (2.94) can be solved (numerically), given the concentration of osmolyte molecules $c_{0}$, the bulk modulus of the hydrogel network $K$, the shear modulus of the hydrogel network $G$, the dynamic viscosity of water $\eta$, and the functional form of the permeability of the hydrogel network $k$, the diffusion coefficient for osmolyte molecules inside the hydrogel $D_{\mathrm{osm}}$, and the osmotic pressure of the osmolyte molecules $\Pi_{\mathrm{osm}}$.

\subsubsection{Dilute polymer solutions}

In the previous section, equation (2.93) was found as equation of motion (EOM) for the deformation $U(R, t)$ of a spherical hydrogel in a polymer solution, with $R$ the position of an infinitesimal portion of the hydrogel network at $t=0$ and $t$ the time. The EOM of $U(R, t)$ is two-way coupled to equation (2.94): the EOM for the number concentration of osmolyte molecules per unit of fluid volume $c(R, t)$. A general exact solution to this sytem of equations lies outside the scope of our capabilities. An exact solution is desirable, however, for it shows the structure of the solution. In this section we will give an exact solution to the EOMs which is valid in the limit of dilute polymer solutions. To solve these EOMs we first assume appropiate functional forms for the permeability of the hydrogel network $k$, the osmolyte diffusion coefficient $D_{\mathrm{osm}}$, and the osmolyte osmotic pressure $\Pi_{\mathrm{osm}}$. Second, we cast them in dimensionless form and finally employ an appropiate perturbation theory to obtain (linearized) EOMs for each order in the perturbation. Afterwards, we exactly solve for the leading order contributions to $c$ and $U$, giving the dominant response of a spherical hydrogel to a surrounding polymer solution. 


\section{Functional forms}

To apply perturbation theory to the equations of motion (EOMs) for the deformation field $U$ and the osmolyte molecules concentration profile $c$, we first consider possible functional forms for the permeability of a polyacrylamide hydrogel $k$, the osmotic pressure $\Pi_{\mathrm{osm}}$ of, for example, $70 \mathrm{kDa}$ dextran, and the osmolyte molecules diffusion coefficient in a polyacrylamide network $D_{\mathrm{osm}}$.

The permeability scales with the volume fraction of network as $\phi_{\mathrm{n}}^{-3 / 2}$ [146], thereby implying $k=k_{0}\left(\frac{\phi_{\mathrm{n}, 0}}{\phi_{\mathrm{n}}}\right)^{3 / 2}$, with $k_{0}$ the permeability of the undeformed network at $t=0$ and $\phi_{\mathrm{n}, 0}$ the volume fraction of hydrogel network at $t=0$. Moreover, the scaled osmolyte molecules osmotic pressure $\tilde{\Pi}_{\text {osm }}$ of monodisperse linear polymer solutions under good solvent conditions can be expressed in terms of a virial expansion in the dilute limit [147]

$$
\tilde{\Pi}_{\mathrm{osm}} \equiv \frac{\Pi_{\mathrm{osm}}}{k_{\mathrm{B}} T c_{0}}=\tilde{c}\left(1+A_{2}^{\prime} \Phi_{\mathrm{osm}, 0} \tilde{c}+A_{3}^{\prime}\left(\Phi_{\mathrm{osm}, 0} \tilde{c}\right)^{2}+A_{4}^{\prime}\left(\Phi_{\mathrm{osm}, 0} \tilde{c}\right)^{3}+\ldots\right),
$$

where $\Pi_{\mathrm{osm}}$ is the osmolyte osmotic pressure, $c_{0}$ is the number concentration of osmolyte molecules in the surrounding solution, $\Phi_{\mathrm{osm}, 0}=v_{\mathrm{osm}} c_{0}$ is the packing fraction of osmolyte molecules in the solution flowing around the hydrogel, $v_{\mathrm{osm}}=\frac{4}{3} \pi R_{\mathrm{g}}^{3}$ is the occupied volume per osmolyte molecule with $R_{\mathrm{g}}$ the radius of gyration, and $\tilde{c} \equiv c / c_{0}$ is the ratio of the fluid concentration of osmolyte inside the hydrogel $c$ to the osmolyte concentration in the surrounding solution $c_{0}$, i.e., the scaled osmolyte molecules concentration. The non-linear coefficients $A_{n}^{\prime}$ are related to the virial coefficients $B_{n}$ by $A_{n+1}^{\prime}=B_{n+1} / v_{\mathrm{osm}}^{n}$. Given that the weight of a typical dextran monomer is $179 \mathrm{Da}$ [141] and that dextran typically consists of a main linear chain with a few small branches [148], we approximate dextran as a linear chain and calculate the first three non-linear coefficients with finite polymerization correction as $A_{2}^{\prime} \approx 1.318, A_{3}^{\prime} \approx 0.564$ and $A_{4}^{\prime} \approx-0.122$ [147]. When comparing the virial expansion with measurements of the osmotic pressure of $70 \mathrm{kDa}$ dextran [123], we observe that theory and experiment have about a factor of two discrepancy. Reasons for this may be that dextran is not a perfectly linear polymer, and the uncertainties in other measured quantities, e.g., the radius of gyration $R_{\mathrm{g}}$. Finally, the osmolyte 
molecules diffusion coefficient $D_{\text {osm }}$ can be written as [128]

$$
D_{\mathrm{osm}}=D_{\mathrm{self}} \frac{c}{k_{\mathrm{B}} T}\left(\frac{\partial \mu_{\mathrm{osm}}}{\partial c}\right)_{T, p}=D_{\mathrm{self}}\left(\frac{\partial \tilde{\Pi}_{\mathrm{osm}}}{\partial \tilde{c}}\right)_{T, p},
$$

where $D_{\text {self }}$ is the self-diffusion coefficient which is generally assumed to be equal to the tracer difusion coefficient $D_{\text {tracer }}$. The tracer diffusion coefficient of osmolyte molecules inside hydrogel networks and polymer solutions is often taken to be of the empirical form [149]

$$
D_{\text {tracer }}(J)=D_{0} e^{-\alpha J^{\beta}}
$$

with $\alpha$ and $\beta$ dimensionless constants of order unity, $D_{0}$ the tracer diffusion coefficient in pure fluid, and $J=\operatorname{det}(\boldsymbol{F})$ the dilution factor of the network with respect to the undeformed state of the hydrogel at $t=0$.

\section{Dimensionless form}

To write the equations of motion in dimensionless form we scale all distances to the typical dimension of length, the original hydrogel radius $R_{0}$, and scale all times to an appropiate dimension of time, the typical time for the fluid to flow through the hydrogel network $\tau_{\mathrm{n}}=a^{2} \eta / M k$, with $\eta$ the dynamic viscosity of water, $K$ the bulk modulus of the hydrogel network and $k$ the permeability of the hydrogel network. The resulting equations are

$$
\begin{array}{r}
\left(\frac{\phi_{\mathrm{n}, 0}}{\phi_{\mathrm{n}}}\right)^{3 / 2} \frac{\partial \tilde{U}}{\partial \tilde{t}}-\frac{1}{\lambda_{r}} \frac{\partial \tilde{\sigma}_{r r}}{\partial \tilde{R}}-\frac{2}{\tilde{R}} \frac{\left(\tilde{\sigma}_{r r}-\tilde{\sigma}_{\theta \theta}\right)}{\lambda_{\theta}}+\epsilon \frac{\partial \tilde{\Pi}_{\text {sol }} / \partial \tilde{c}}{\lambda_{r}} \frac{\partial \tilde{c}}{\partial \tilde{R}} \\
\Phi_{\mathrm{s}} \frac{\partial \tilde{c}}{\partial \tilde{t}}-\frac{\tau_{\mathrm{n}}}{t_{\mathrm{sol}}} \frac{1}{\tilde{R}^{2}} \frac{\partial}{\partial \tilde{R}}\left(\tilde{R}^{2} \frac{\lambda_{\theta}^{2}}{\lambda_{r}} \frac{D_{\mathrm{osm}}}{D_{\text {tracer }}(1)} \frac{\partial \tilde{c}}{\partial \tilde{R}}\right)+\tilde{c} \frac{1}{\tilde{R}^{2}} \frac{\partial}{\partial \tilde{R}}\left(\left(\lambda_{\theta} \tilde{R}\right)^{2} \frac{\partial \tilde{U}}{\partial \tilde{t}}\right)
\end{array}
$$

where $\tilde{U}=U / a$ is the scaled deformation field, $\tilde{t}=t / \tau_{\mathrm{n}}$ is the scaled time, $\tilde{R}=R / a$ is the scaled original position of the hydrogel network, $t_{\text {sol }}=a^{2} / D_{\text {tracer }}(1)$ is the ratio of the original hydrogel radius squared to the diffusion constant of osmolyte molecules in the hydrogel in the undeformed state, i.e., the typical time for osmolyte molecules to diffuse through the hydrogel network, $\tilde{\sigma}_{i i}=\sigma_{i i}^{\prime} / M$ (no summation) are the scaled Terzaghi effective stresses for $i=r, \theta$, and the principle stretches are given by $\lambda_{r}=1+\partial \tilde{U} / \partial \tilde{R}$ and $\lambda_{\theta}=1+\tilde{U} / \tilde{R}$. Furthermore, $\tilde{c}=c / c_{0}$ is the concentration of osmolyte molecules per unit of fluid volume scaled to the concentration of 
osmolyte molecules in the surrounding fluid and $\epsilon=k_{\mathrm{B}} T c_{0} / K$ is the ratio of a hypothetical van 't Hoff osmotic pressure of the surrounding solution to the bulk modulus of the hydrogel $K$, i.e., $\epsilon$ is a measure for the osmotic strength of the surrounding polymer solution relative to the resistance to (de)swelling of the hydrogel network. The scaled stresses of the polymer network are given by

$$
\begin{aligned}
& J \tilde{\sigma}_{r r}=\ln \lambda_{r}+2 \frac{\Lambda}{M} \ln \lambda_{\theta}, \\
& J \tilde{\sigma}_{\theta \theta}=\frac{\Lambda}{M} \ln \lambda_{r}+\left(1+\frac{\Lambda}{M}\right) \ln \lambda_{\theta},
\end{aligned}
$$

where $J=\lambda_{r} \lambda_{\theta}^{2}$ and $\Lambda / M=\nu /(1-\nu)$, with $\nu$ the Poisson ratio.

Summarizing, the parameters governing the dimensionless equations are the relative strength of the polymer solution $\epsilon$, the ratio of the typical gel deformation time to the typical osmolyte molecules diffusion time $\tau_{\mathrm{n}} / \tau_{\mathrm{osm}}$, the Poisson ratio $\nu$, the phenomenological coefficients determining the diffusion of dextran inside the polyacrylamide network $\alpha$ and $\beta$, the volume fraction of osmolyte molecules in the dextran solution $\Phi_{\mathrm{osm}, 0}$, and the dextran osmotic pressure virial coefficients $B_{i}$, with $i=1,2, \ldots$.

\section{Perturbation theory}

From the EOMs, equation (2.102) and (2.103), it can be observed that $\epsilon$ determines the size of the $\tilde{c}$ source term in equation (2.102) and that the source term in equation (2.103) is proportional to $\tilde{U}$. To solve the EOMs in the limit of dilute polymer solutions, $\epsilon=k_{\mathrm{B}} T c_{0} / K \ll 1$, we employ a perturbation expansion in $\epsilon$. For $\epsilon=0$, i.e., no osmolyte, we have $\tilde{U}=0$ for all times because there is no source term in equation (2.102), because there is no driving term in the boundary conditions, and because $\tilde{U}=0$ at $\tilde{t}=0$. Therefore, we write

$$
\begin{aligned}
\tilde{U} & =\epsilon \tilde{U}^{(1)}+\epsilon^{2} \tilde{U}^{(2)}+\ldots, \\
\tilde{c} & =\tilde{c}^{(0)}+\epsilon \tilde{c}^{(1)}+\epsilon^{2} \tilde{c}^{(2)}+\ldots,
\end{aligned}
$$

with $\tilde{U}^{(i)}$ and $\tilde{c}^{(i)}$ the contribution to $\tilde{U}$ and $\tilde{c}$ of the $i$-th order in $\epsilon$. For notational convenience, we drop all tildes in the rest of the Appendix until they are restored. Because the leading order term in the expansion for $U$ is of order $\epsilon$, we anticipate 
that the source term in equation (2.103) is small relative to the other terms. Next, the importance of the non-ideal terms in equation (2.99) is determined by $\Phi_{\mathrm{osm}, 0}$ which can be written as $\Phi_{\mathrm{osm}, 0}=v_{\mathrm{osm}} c_{0}=\epsilon \frac{4}{3} \pi R_{\mathrm{g}}^{3} \frac{K}{k_{\mathrm{B}} T}$ upon using the definition of $\epsilon$. Assuming the polyacrylamide network to be isotropic and homogeneous in its elastic constants, we have $K=G \frac{2(1+\nu)}{3(1-2 \nu)}$, with $\nu$ the Poisson ratio. Also, classical network theory [150] gives $G=n k_{\mathrm{B}} T$, with $n$ the cross-link density which is assumed to be proportional to the number of chains per unit volume. Taking the volume per chain to be approximately equal to the cube of the mesh size $\zeta^{3}$, we obtain $\Phi_{\mathrm{osm}, 0} \approx \epsilon \frac{4}{3} \pi\left(R_{\mathrm{g}} / \zeta\right)^{3} \frac{2(1+\nu)}{3(1-2 \nu)} \approx 0.18 \epsilon$ for a $70 \mathrm{kDa}$ dextran radius of gyration of $R_{\mathrm{g}} \approx 6 \mathrm{~nm}$ [141, 142], a typical mesh size of the polyacrylamide network of $\zeta \approx 50 \mathrm{~nm}$ [143] and a Poisson ratio of $\nu \approx 0.48$ [136]. From these considerations we may conclude that $\Phi_{\mathrm{osm}, 0}$ can be determined by the relative strength of the polymer solution $\epsilon$.

Given the proportionality of $\Phi_{\mathrm{osm}, 0}$ to $\epsilon$, and equations (2.99), (2.100), (2.101), (2.106) and (2.107), we write equation (2.102) and (2.103), as well as the boundary conditions found in section 2.6.10, as a power series in $\epsilon$. Because $\epsilon$ is small and because it can be chosen arbitrarily, we require for each order in $\epsilon$ the appurtenant coefficient on both sides of each equation to be equal. We obtain from the zeroth order of equation (2.103) a Fickian diffusion equation for the zeroth order osmolyte concentration $c^{(0)}$

$$
\Phi_{\mathrm{s}, 0} \frac{\partial c^{(0)}}{\partial t}-\frac{\tau_{\mathrm{n}}}{\tau_{\mathrm{osm}}} \frac{1}{R^{2}} \frac{\partial}{\partial R} R^{2} \frac{\partial c^{(0)}}{\partial R}=0,
$$

with $c^{(0)}=1$ at $R=1$ for $t>0, \partial c^{(0)} / \partial R=0$ at $R=0$ for $t>0$, and $c^{(0)}=0$ at $t=0$ for $\left.\left.R \in\right] 0,1\right]$. Fickian diffusion is to be expected, because the zeroth order contribution to $U$ corresponds to no deformation, i.e., $U^{(0)}=0$. Furthermore, from the first order equation from equation (2.103), the force balance condition at the surface of the hydrogel and the initial condition for $U$, we find the following equation for $U^{(1)}$

$$
\frac{\partial U^{(1)}}{\partial t}-\frac{\partial}{\partial R} \frac{1}{R^{2}} \frac{\partial}{\partial R} R^{2} U^{(1)}+\frac{\partial c^{(0)}}{\partial R}=0,
$$

with $M \partial U^{(1)} / \partial R+2 \Lambda U^{(1)} / R=0$ at $R=1$ for $t>0, U^{(1)}=0$ at $R=0$ for $t>0$, and $U^{(1)}=0$ at $t=0$ for $\left.\left.R \in\right] 0,1\right]$. The evolution of $U^{(1)}$ satisfies a diffusivelike EOM and is driven by the gradient of the zeroth order osmolyte concentration profile, implying differences in the osmotic pressure in the hydrogel to cause deformation. The solution to this equation gives the dominant response of a hydrogel to 
a dilute polymer solution. The higher order EOMs are all linear diffusion-like equations with increasingly complex source terms. The source term in the $c^{(1)} \mathrm{EOM}$, for example, is composed of terms of combinations of $c^{(0)}$ and $U^{(1)}$ which incorporate the dominant variation of the diffusion coefficient and the lowest order influence of dilution of the hydrogel network through the source term in equation (2.103). For reference, equation (2.108) and (2.109) can also be found from the theory of irreversible processes applied to the movement of osmolyte molecules through a matrix in the limit of dilute polymer solutions, see the work of Mauck et al. [132].

\section{Exact solution}

In the previous paragraph the equations of motion for the leading order contributions to the perturbative expansion of the hydrogel deformation field $U$ and the osmolyte molecules concentration profile $c$ in the limit of dilute polymer solution were found. Their solution is found by first solving equation (2.108) with its accompanying boundary and initial conditions [135] to find

$$
c^{(0)}(R, t)=1-\sum_{n=1}^{\infty} 2(-1)^{n+1} \mathrm{j}_{0}(\pi n R) \exp \left(-(\pi n)^{2} \frac{\tau_{\mathrm{n}}}{\Phi_{\mathrm{s}, 0} \tau_{\mathrm{osm}}} t\right),
$$

where $\mathrm{j}_{\mathrm{o}}$ is the zeroth order spherical Bessel function of the first kind.

Equation (2.109) with its appurtenant boundary and initial conditions can be solved by using an integral transform, the Hankel transform. We find an orthogonal set of eigenfunctions, in order to transform the $R$-coordinate, by considering an auxiliary problem [135]. This problem is similar to the spacial equation one obtains by seperation of variables, is given by

$$
\begin{aligned}
\frac{\partial}{\partial R}\left(\frac{1}{R^{2}} \frac{\partial}{\partial R}\left(R^{2} \psi\right)\right)+\beta^{2} \psi & =0, \\
M \frac{\partial \psi}{\partial R}+2 \Lambda \frac{\psi}{R} & =0, \text { at } R=1, \\
\psi & =0, \text { at } R=0,
\end{aligned}
$$

and has the following solutions

$$
\psi_{m}(R)=\frac{1}{N_{m}^{1 / 2}} \mathrm{j}_{1}\left(\beta_{m} R\right), m=1,2, \ldots
$$


where $\mathrm{j}_{1}$ is a spherical Bessel function of the first kind of order one and $N_{m}^{1 / 2}$ is a normalization such that the solutions form an orthonormal set of functions, founding a complete basis on $R \in] 0,1]$. We neglect Bessel functions of the second kind because they diverge at $R=0$, and the boundary condition at $R=0$ is fulfilled because $\mathrm{j}_{1}(0)=0$. The eigenvalues $\beta_{m}$ are determined by the positive solutions of the transcendental equation

$$
4 \frac{(1 / 2-\nu)}{1-\nu} \mathrm{j}_{1}\left(\beta_{m}\right)=\beta_{m} \mathrm{j}_{0}\left(\beta_{m}\right)
$$

implying $\beta_{m}$ to be determined by the Poisson ratio $\nu$ alone. The norm is given by

$$
N_{m}=\int_{0}^{1} \mathrm{~d} R R^{2} \mathrm{j}_{1}^{2}\left(\beta_{m} R\right)=\frac{2 \cos \left(2 \beta_{m}\right)+\beta_{m} \sin \left(2 \beta_{m}\right)+2\left(\beta_{m}^{2}-1\right)}{4 \beta_{m}^{4}} .
$$

The Hankel transform of an arbitrary function $l(R)$ with $R \in[0,1]$ is given by

$$
\bar{l}_{m}=\int_{0}^{1} \mathrm{~d} R R^{2} \psi_{m}(R) l(R)
$$

and its inverse is

$$
l(R)=\sum_{m=1}^{\infty} \bar{l}_{m} \psi_{m}(R)
$$

where the sum is over all positive eigenvalues, given by equation (2.115). Therefore, we have for the transform of the first order contribution $U_{m}^{(1)}$

$$
U_{m}^{(1)}(t)=\int_{0}^{1} \mathrm{~d} R R^{2} U^{(1)}(R, t) \psi_{m}
$$

and upon transforming equation (2.109) we find

$$
\frac{\partial U_{m}^{(1)}}{\partial t}+\beta_{m}^{2} U_{m}^{(1)}=-\int_{0}^{1} \mathrm{~d} R R^{2} \psi_{m} \partial c^{(0)} / \partial R \equiv s_{m}
$$

where we used double partial integration and the boundary terms vanish, because there is no net external force acting at the boundary of the hydrogel. We write $\partial c^{(0)} / \partial R$ as

$$
\partial c^{(0)} / \partial R=-\sum_{n=1}^{\infty} 2(-1)^{n} \pi n \mathrm{j}_{1}(\pi n R) \exp \left(-(\pi n)^{2} \frac{\tau_{\mathrm{n}}}{\Phi_{\mathrm{s}, 0} \tau_{\mathrm{osm}}} t\right)
$$


so in $s_{m}$, on the righthand side of equation (2.120), we have for any $n, m=$ $1,2, \ldots$

$$
\int_{0}^{1} \mathrm{~d} R R^{2} \mathrm{j}_{1}\left(\beta_{m} R\right) \mathrm{j}_{1}(\pi n R)=(-1)^{n} \frac{\gamma_{m n}}{\pi n},
$$

provided that $\beta_{m} \neq \pi n$, which requires $\nu \neq 1 / 2$, and where

$$
\gamma_{m n} \equiv \frac{\sin \left(\beta_{m}\right)}{\beta_{m}^{2}-(\pi n)^{2}}
$$

We can interpret $\gamma_{m n}$ as the spacial interference of osmolyte molecules diffusion and gel deformation modes. For $s_{m}$ we obtain

$$
s_{m}=\sum_{n=1}^{\infty} \frac{2}{N_{m}^{1 / 2}} \gamma_{m n} \exp \left(-(\pi n)^{2} \frac{\tau_{\mathrm{n}}}{\Phi_{\mathrm{s}, 0} \tau_{\mathrm{osm}}} t\right) .
$$

Next, by integrating equation (2.120) in time and by using that $U_{m}=0$ at $t=0$ because $U(R, 0)=0$, we find

$$
\begin{aligned}
U_{m}^{(1)} & =e^{-\beta_{m}^{2} t} \int_{0}^{t} \mathrm{~d} t^{\prime} s_{m n}\left(t^{\prime}\right) e^{\beta_{m}^{2} t^{\prime}} \\
& =\sum_{n=1}^{\infty} \frac{2}{N_{m}^{1 / 2}} \gamma_{m n} e^{-\beta_{m}^{2} \tau} \int_{0}^{t} \mathrm{~d} t^{\prime} e^{t^{\prime}\left(\beta_{m}^{2}-(\pi n)^{2} \tau_{\mathrm{n}} / \tau_{\mathrm{osm}} \Phi_{\mathrm{s}, 0}\right)} \\
& \equiv \sum_{n=1}^{\infty} \frac{2}{N_{m}^{1 / 2}} \gamma_{m n} \Gamma_{m n}(t)
\end{aligned}
$$

with $\Gamma_{m n}$ the temporal interference between osmolyte molecules diffusion and gel deformation modes. The temporal interference $\Gamma_{m n}$ can be calculated as

$$
\Gamma_{m n}(t)=\frac{\exp \left(-\beta_{m}^{2} t\right)-\exp \left(-(\pi n)^{2}\left(\tau_{\mathrm{n}} / \tau_{\mathrm{osm}} \Phi_{\mathrm{s}, 0}\right) t\right)}{(\pi n)^{2} \tau_{\mathrm{n}} / \tau_{\mathrm{osm}} \Phi_{\mathrm{s}, 0}-\beta_{m}^{2}} .
$$

Finally, with the inverse Hankel transform, we find $U^{(1)}(R, t)$ as

$$
U^{(1)}(R, t)=\sum_{n, m=1}^{\infty} \frac{2}{N_{m}} \gamma_{m n} \mathrm{j}_{1}\left(\beta_{m} R\right) \Gamma_{m n}(t) .
$$

Recalling that the above result was derived with dimensionless quantities and upon restoring the tildas for the dimensionless quantities defined in paragraph 2.6.11, we 
obtain the dimensionfull result for $U(R, t)$ and $c(R, t)$ up to leading order in the relative strength of the polymer solution $\epsilon$ as

$$
\begin{aligned}
& U(R, t)=a \epsilon \sum_{n, m=1}^{\infty} \frac{2}{N_{m}} \gamma_{m n} \mathrm{j}_{1}\left(\beta_{m} \frac{R}{a}\right) \frac{\exp \left(-\beta_{m}^{2} t / \tau_{\mathrm{n}}\right)-\exp \left(-(\pi n)^{2} t / \tau_{\mathrm{osm}} \Phi_{\mathrm{s}, 0}\right)}{(\pi n)^{2} \tau_{\mathrm{n}} / \tau_{\mathrm{osm}} \Phi_{\mathrm{s}, 0}-\beta_{m}^{2}} \\
& c(R, t)=c_{0}\left[1-\sum_{n=1}^{\infty} 2(-1)^{n+1} \mathrm{j}_{0}\left(\pi n \frac{R}{a}\right) \exp \left(-(\pi n)^{2} \frac{t}{\Phi_{\mathrm{s}, 0} \tau_{\mathrm{osm}}}\right)\right]
\end{aligned}
$$

From this result we observe that the leading order response of a hydrogel to a dilute polymer solution, i.e., the deformation field $U$, is parametrized by: the initial $t=0$ hydrogel radius $R_{0}$, the typical time for fluid to flow through the hydrogel network $\tau_{\mathrm{n}}$, the typical time for the osmolyte molecules to diffuse through the hydrogel network $\tau_{\text {osm }}$, the strength of the dilute polymer solution $\epsilon=k_{\mathrm{B}} T c_{0} / K$, and the Poisson ratio $\nu$ which determines $N_{m}, \gamma_{m n}$, and $\beta_{m}$ through equation (2.115), and therefore determines the way the network responds to deformations. The coefficients $A_{i}^{\prime}$ determining both the non-ideal osmotic pressure contributions and the diffusion coefficient $D_{\text {osm }}$, and the phenomenological constants $\alpha$ and $\beta$ which determine the influence of gel deformation on osmolyte molecules diffusion in the hydrogel do not enter explicitly into the leading order contributions. These are signals that the leading order contributions effectively treat the permeability as a constant, approximate the osmotic pressure with van 't Hoffs law, and take the diffusion coefficient of osmolyte molecules as a constant throughout the process of compression and reswelling.

The Hankel modes contributing to the deformation field $U$ turn out to be biexponential. The reason for this is the following. The leading order contribution to the concentration profile of osmolyte molecules $c^{(0)}$ satisfies a Fickian diffusion equation, i.e., a first order equation in time, thereby giving rise to exponential relaxation of the concentration profile. The equation of motion for the leading order hydrogel deformation $U^{(1)}$ contains $c^{(0)}$ in its source term and is diffusive-like. Therefore, the response of the hydrogel is an interplay between two diffusive relaxation processes, i.e., the diffusion of osmolyte molecules and the flow of solvent through the hydrogel network, implying its response to be biexponential. 


\section{Chapter 3}

\section{Poroelasticity of (bio)polymer networks during compression: theory and experiment}

The results of this chapter are contained in ${ }^{1}$ :

M.T.J.J.M. Punter, B.E. Vos, B.M. Mulder \& G.H. Koenderink. "Poroelasticity of (bio)polymer networks during compression: theory and experiment". In: Soft Matter (2020) Accepted Manuscript.

\footnotetext{
${ }^{1}$ The experiments reviewed in this chapter have been designed and carried out by Bart E. Vos and Gijsje H. Koenderink from AMOLF (The Netherlands).
} 


\section{Abstract}

Soft living tissues like cartilage can be considered as biphasic materials comprised of a fibrous complex biopolymer network and a viscous background liquid. Here, we show by a combination of experiment and theoretical analysis that both the hydraulic permeability and the elastic properties of (bio)polymer networks can be determined with simple ramp compression experiments in a commercial rheometer. In our approximate closed-form solution of the poroelastic equations of motion, we find the normal force response during compression as a combination of network stress and fluid pressure. Choosing fibrin as a biopolymer model system with controllable pore size, measurements of the full time-dependent normal force during compression are found to be in excellent agreement with the theoretical calculations. The inferred elastic response of large-pore $(\mu \mathrm{m})$ fibrin networks depends on the strain rate, suggesting a strong interplay between network elasticity and fluid flow. Phenomenologically extending the calculated normal force into the regime of nonlinear elasticity, we find strain-stiffening of small-pore (sub- $\mu \mathrm{m}$ ) fibrin networks to occur at an onset average tangential stress at the gel-plate interface that depends on the polymer concentration in a power-law fashion. The inferred permeability of small-pore fibrin networks scales approximately inverse squared with the fibrin concentration, implying with a microscopic cubic lattice model that the number of protofibrils per fibrin fiber cross-section decreases with protein concentration. Our theoretical model provides a new method to obtain the hydraulic permeability and the elastic properties of biopolymer networks and hydrogels with simple compression experiments, and paves the way to study the relation between fluid flow and elasticity in biopolymer networks during dynamical compression. 


\subsection{Introduction}

Soft biopolymer networks have essential functions in living cells [151, 152], the extracellular matrix $[153,154]$ and the process of blood coagulation [94, 155]. Their mechanical properties are determined by the network's hydraulic permeability and (visco)elastic properties.

The permeability of biopolymer networks determines mass transport in soft tissues $[92,156,157]$, the dynamic behaviour of cells $[85,158]$ and the (dis)functioning of blood clots in hemostasis and thrombosis [159-161]. Conventionally, the permeability of porous materials is inferred from the measured flow rate of a liquid through the material $[146,162]$. There are alternative approaches to measure the permeability of porous materials, such as microfluidic devices [32], but sticky biopolymer gels are prone to block such devices. Another complicating factor is that separate measurements on biopolymer gels are required for a characterization of their elastic properties. The elastic properties of biopolymer networks are essential for the physiological function of tissues and in wound healing [100]. For instance, arteries need to be extendable to provide blood pressure capacitance and pulse smoothing in the blood circulation [163], and blood clots are required to be resilient structural scaffolds in wound healing [164]. The elastic properties of biopolymer networks have been studied extensively in shear $[100,165]$, extension $[96,166]$ and static compression [167]. The dynamic response during compression, however, remains largely unexplored $[168,169]$.

Seeking to establish both the permeability and the elastic properties of dynamically compressed biopolymer networks in a single test, we consider slow ramp compression tests with a commercial rheometer. For interpretation of the measured normal force, we use the theory of poroelasticity [23, 27, 28], as applied to polymer gels [33]. Previously, this theory has been applied successfully to other systems, e.g. interstitial fluid flow through (mineralized) bone tissue [170, 171]. In short, this theory constructs a stress field in a poroelastic material whose physical origin is twofold: elastic stresses from the deformed network, and pressure from the fluid. Network stress and fluid pressure are tightly coupled: when a fast compressive deformation is applied, for example, stresses are generated in the network because it is forced to deform in a volume-conserving manner. By equilibrium conditions on the overall stress field, pressure is induced in the fluid, prompting fluid flow through the porous material by 

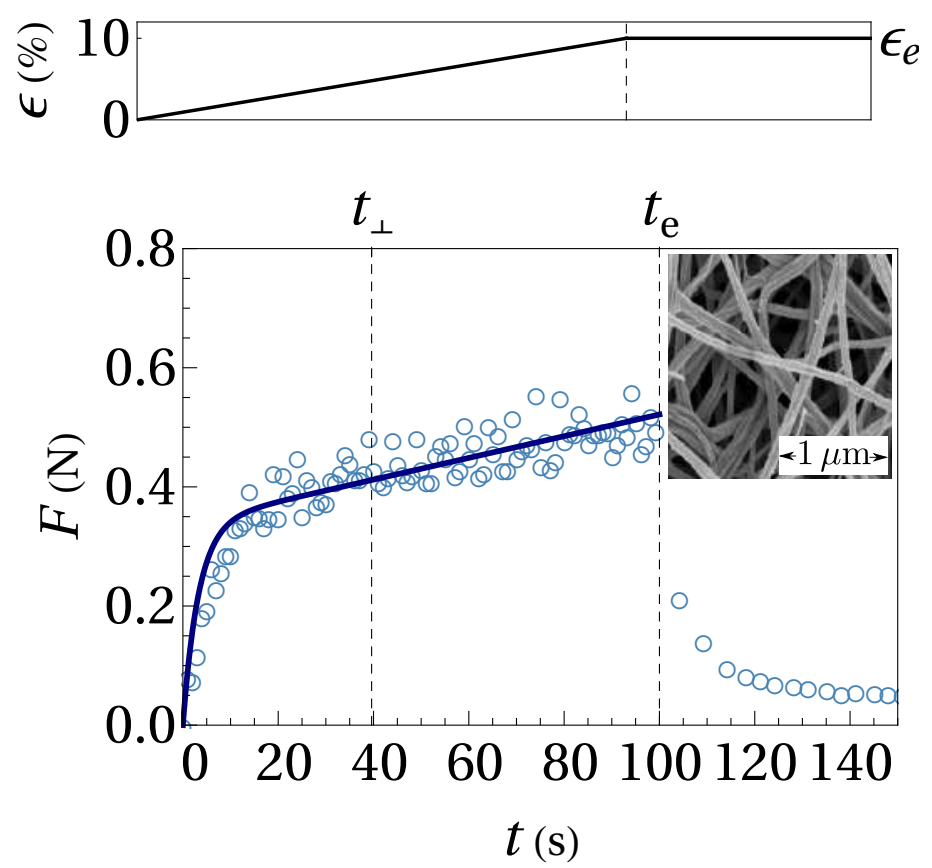

Figure 3.1: The measured normal force $F$ (blue circles) of a large-pore fibrin gel (mesh size $\zeta \sim 1 \mu \mathrm{m}$ and fibrinogen concentration $c=2 \mathrm{mg} / \mathrm{mL}$ ) with initial radius $a=$ $20 \mathrm{~mm}$ and height $h=1 \mathrm{~mm}$ in response to ramp compression in $t_{\mathrm{e}}=100 \mathrm{~s}$ up to $10 \%$ engineering strain $\epsilon_{\mathrm{e}}$. During the pressurizing time $t_{\perp}=40 \mathrm{~s}$ the fluid pressure builds up to its maximal value. Subsequently, the compression of the fibrin network keeps increasing the normal force. After compression, the fluid pressure contribution decreases to zero; the residual normal force consists only of a static network response. Assuming the independently measured shear modulus $G_{0}=139 \mathrm{~Pa}$ to be constant during compression, a fit of the calculated normal force in equation (3.4) (blue curve) gives the permeability of the fibrin network as $k=(1.26 \pm 0.03) \cdot 10^{-1} \mu \mathrm{m}^{2}$ and its longitudinal modulus as $M=K+4 G_{0} / 3=1.5 \pm 0.1 \mathrm{kPa}$, with $K$ the bulk modulus and \pm denotes the estimation uncertainty. Inset: scanning electron microscopy image of a large-pore fibrin gel with $c=2 \mathrm{mg} / \mathrm{mL}$.

Darcy's law [28].

We propose an approximate closed-form solution to the poroelastic equations of motion from which we calculate the normal force, during ramp compression, of a disklike cylindrical gel bonded to the plates of a parallel-plate rheometer. The theoretical calculation of the normal force allows us to infer the permeability and elastic properties of a biopolymer network, or any other gel bonded to the plates. The calculated 
normal force separates the contribution of the fluid and the gel network to the measured normal force, describing the full temporal evolution during ramp compression, which, to the best of our knowledge, is lacking in literature, see for example Kim et al. [169]. To test the approximate solution, we use covalently cross-linked fibrin gels as a model system. Fibrin is a fibrous protein structure that is the main structural component of blood clots. The formation of a fibrin gel starts with thrombin cleaving fibrinopeptides from dissolved fibrinogen molecules to obtain fibrin monomers. The fibrin monomers then assemble in a half-staggered manner, forming elongated protofibrils of two molecules thick. These protofibrils interconnect to form the relatively thick fibrin fibers that constitute the fibrin network [94]. The fibers themselves are immersed in fluid, making a hydrogel with a solid volume fraction of typically less than $1 \%$.

Fibrin gels are convenient experimental model systems because their permeability can be manipulated through the polymerization conditions, with pore sizes that can be either several tens of nanometers or a few microns [172]. We perform ramp compression tests on disk-like cylindrical fibrin gels in which we vary the fibrinogen concentration, the amount of strain, the strain rate and the dimensions of the gel. The normal force response of fibrin gels during compression can be explained with our theoretical solution, accounting for the time-dependent build-up of fluid pressure, see Figure 3.3-3.1. Since for large-pore fibrin gels the vast majority of fluid pressure builds up in only a few seconds, we also considered small-pore fibrin gels having a prolonged phase of pressure build-up, see Figure 3.2.

We find that the flow of fluid through large-pore fibrin networks has a strong influence on its mechanical response under both small (5-10\% compressive strain) and large $(\leq 80 \%)$ compression. Phenomenologically extending the theoretical solution to include strain-stiffening during compression, we show that strain-stiffening of small-pore fibrin networks occurs during pressure build-up around an onset average tangential stress at the gel-plate interface, similar as in shear rheology [100]. The onset stress depends on the fibrinogen concentration in a power-law fashion. Fluid flow through the fibrin network inhibits strain-stiffening, implying a nontrivial interplay between network elasticity and fluid flow. The permeability of small-pore fibrin networks is found to scale approximately with the inverse squared of the fibrinogen concentration, implying with a microscopic cubic lattice model that the number of protofibrils per fibrin fiber cross-section decreases with the overall fibrinogen con- 


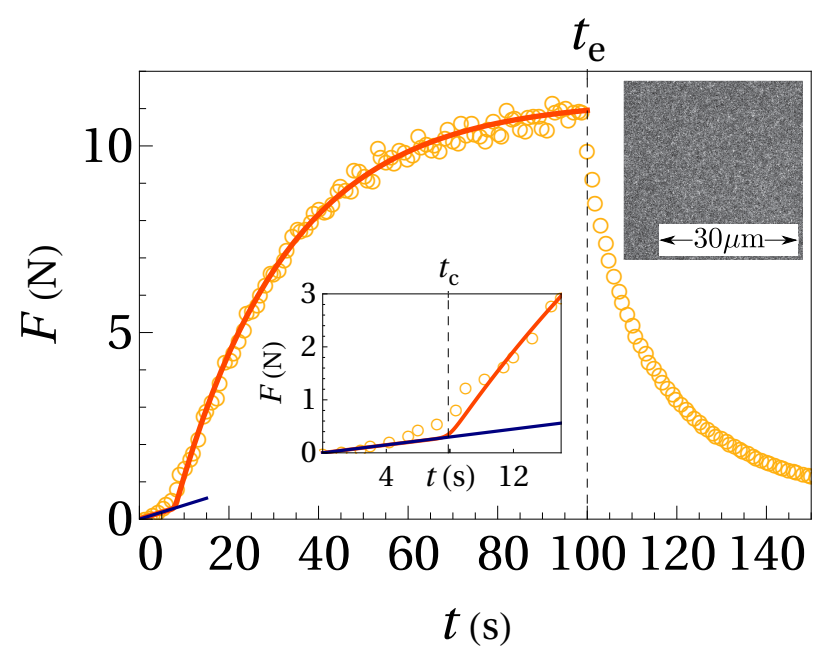

Figure 3.2: The measured normal force $F$ (orange circles) of a small-pore fibrin gel (mesh size $\zeta \sim 0.1 \mu \mathrm{m})$ probed under identical conditions as the large-pore gel of Figure 3.1. The relatively small pores cause a large pressurizing time $t_{\perp}=300 \mathrm{~s}$ and fluid pressure. Before the onset time of strain-stiffening $t_{\mathrm{c}}=7.9 \pm 0.3 \mathrm{~s}$, with \pm denoting the estimation uncertainty, the normal force follows the time dependence expected for a volumeconserving linear elastic solid with the measured initial shear modulus $G_{0}=49 \mathrm{~Pa}$ (blue line in the inset). Fitting equation (3.4), combined with (3.7), we find the permeability as $k=(3.86 \pm 0.15) \cdot 10^{-3} \mu \mathrm{m}^{2}$, the augmented shear modulus after stiffening as $G_{\mathrm{c}}=589 \pm 19 \mathrm{~Pa}$ and the longitudinal modulus as $M=0 \pm 5 \mathrm{kPa}$ (red curve). The latter could not be estimated due to the small contribution of network elasticity to the normal force. Top right inset: single 2D fluorescence microscopy image of a fine fibrin network. As the mesh size of this network is smaller than the diffraction limit of light [172], the network cannot be resolved with fluorescence microscopy.

centration in the gel.

After introducing the compression experiments on fibrin gels, we develop the theoretical background, the theoretical calculation for the normal force, and its phenomenological extensions. The strain rate dependence of the elastic properties of large-pore fibrin networks is discussed, as well as the onset stress for strain-stiffening and the inferred permeability of small-pore fibrin networks. 

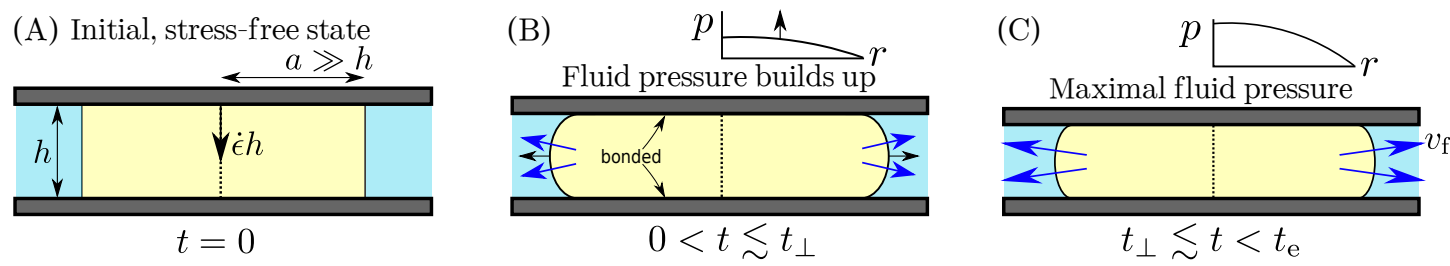

Figure 3.3: Two compression phases of (A) an initially stress-free cylindrical fibrin gel (yellow) of radius $a$ and height $h$ with high aspect ratio $S \equiv a / h \gg 1$ (Figure not on scale). The gel is ramp compressed in a parallel-plate rheometer with the upper plate (gray) having a constant velocity $\dot{\epsilon} h$, where $\dot{\epsilon}$ is the strain rate. B) As compression commences, the fibrin network starts to bulge out (black arrows) because the gel is bonded to the plates, causing the fluid pressure $p$ to build up in a pressurizing time $t_{\perp}$. The build-up proceeds until the fluid outflow velocity $v_{\mathrm{f}}$ (blue arrows) due to the fluid pressure gradient, $v_{\mathrm{f}} \propto-\partial p / \partial r$ with $r$ the radial coordinate in the fibrin gel, is such that the outward bulging of the gel network, which induces the fluid pressure, stabilizes. C) Afterwards, the gel is compressed further at maximal fluid pressure until at time $t_{\mathrm{e}}$ the compression stops.

\subsection{Materials and Methods}

\subsubsection{Materials}

Human plasma fibrinogen (contains naturally occuring Factor XIIIa; plasminogen, von Willebrand Factor and Fibronectin depleted) and human $\alpha$-thrombin were obtained in lyophilized form from Enzyme Research Laboratories (Swansea, United Kingdom). All chemicals were obtained from Sigma Aldrich (Zwijndrecht, The Netherlands). Fibrinogen was dissolved in water at $37^{\circ} \mathrm{C}$ for $15 \mathrm{~min}$ to its original concentration (approximately $13 \mathrm{mg} / \mathrm{ml}$ ) and dialysed against fibrin buffer containing $20 \mathrm{mM}$ 4-(2-hydroxyethyl)-1-piperazineethanesulfonic acid (HEPES) and $150 \mathrm{mM} \mathrm{NaCl}$ at a $\mathrm{pH}$ of 7.4 , and stored at $-80^{\circ} \mathrm{C}$. Prior to use, the fibrinogen was quickly thawed at $37^{\circ} \mathrm{C}$, and then diluted in a final assembly buffer containing $20 \mathrm{mM}$ HEPES, $150 \mathrm{mM} \mathrm{NaCl}$ and $5 \mathrm{mM} \mathrm{CaCl}_{2}$ (large-pore gels). Dense networks (small-pore gels) with an average pore size of $0.08 \mu \mathrm{m}$, as determined by light scattering measurements [172, 173], were obtained in small-pore-gel assembly buffer (400 $\mathrm{mM} \mathrm{NaCl}, 3.2 \mathrm{mM} \mathrm{CaCl}_{2}$ and $50 \mathrm{mM}$ Tris- $\left.\mathrm{HCl}\right)$ at a $\mathrm{pH}$ of 8.5 [174].

Fibrin polymerization was initiated by the addition and quick mixing of $0.5 \mathrm{U} / \mathrm{ml}$ of 
thrombin from a $20 \mathrm{U} / \mathrm{ml}$ thrombin stock, kept on ice for a maximum of 24 hours. After addition of thrombin, the mixture was quickly transferred to the rheometer to allow in situ polymerization. During polymerization, we measured the linear elastic shear modulus $G^{\prime}$ of the fibrin gels by measuring the stress response to a small oscillatory shear strain with an amplitude of $0.5 \%$ and a frequency of $0.5 \mathrm{~Hz}$. In this way the shear modulus just before compression $G_{0}$ was determined.

\subsubsection{Compression experiments}

To measure the normal force produced by fibrin gels under ramp uniaxial compression, we use an Anton Paar rheometer (Physica MCR 501, Graz, Austria) to compress disk-like fibrin gels, confined between two impermeable surfaces: a stainless steel bottom plate and a steel top plate, separated by an initial gap $h$ of 1 or $0.5 \mathrm{~mm}$. We used top plates with diameters of either 20 or $40 \mathrm{~mm}$. The plates of the rheometer were held at $37^{\circ} \mathrm{C}$ throughout the experiment. To provide external hydrostatic pressure and to prevent the formation of a fibrin membrane at the free boundary of the gel, we immersed the gel in mineral oil [175].

In the analysis of the time-dependent normal force, the liquid can safely be assumed to be incompressible at the pressures we apply: the force transducer in the rheometer can apply normal forces up to $50 \mathrm{~N}$, implying the maximum engineering stress to be of the order of $10 \mathrm{kPa}$. To verify that in the compression experiments only liquid is expelled while the network remains intact, we collected the expelled liquid and confirmed by spectrophotometric measurements of the absorbance at 280 and $320 \mathrm{~nm}$ that no protein was present.

\subsection{Theoretical framework}

To calculate the normal force response of a fibrin gel under compression we use the theory of linear poroelasticity which rests on the following three principles [23, 27, 28]. 1). Assuming fluid flow through the fibrin network to be in the regime of low Reynolds number, and because the gel is immersed in fluid, we can neglect, respectively, inertia and gravity, implying the overall force balance of the fibrin gel 
to read [27]

$$
\nabla \cdot\left(\boldsymbol{\sigma}^{\prime}-p \mathbb{1}\right)=\mathbf{0}
$$

where $\sigma^{\prime}$ is the Terzaghi effective stress of the fibrin network: the network stress relative to the pressure $p$ of the permeating fluid. We take the effective stress to be that of a linear elastic isotropic homogeneous solid with the bulk modulus $K$ and the shear modulus $G$ as elastic constants. 2) Assuming the fibrin fibers and the fluid both to be individually incompressible, we find from mass conservation the incompressibility condition for a fibrin gel as [33]

$$
\nabla \cdot \boldsymbol{V}=0
$$

where $\boldsymbol{V} \equiv \phi_{\mathrm{f}} \boldsymbol{v}_{\mathrm{f}}+\phi_{\mathrm{n}} \boldsymbol{v}_{\mathrm{n}}$ is the gel velocity: a sum of the local volume-averaged velocity $\boldsymbol{v}_{i}$ of the fluid and the fibrin network weighted with their respective volume fractions $\phi_{i}$. 3) Finally, in the low Reynolds number regime, Darcy's law governs the flow of fluid through the fibrin network [27]

$$
\phi_{\mathrm{f}}\left(\boldsymbol{v}_{\mathrm{f}}-\boldsymbol{v}_{\mathrm{n}}\right)=-\frac{k}{\eta} \nabla p
$$

where $k$ is the permeability of the fibrin network and $\eta$ the dynamic viscosity of the fluid, which we take to be equal to that of pure water at $37^{\circ} \mathrm{C}$.

For a compressed fibrin gel with initial radius $a$ and height $h$, experiencing no friction with the rheometer plates, equation (3.1)-(3.3) can be solved exactly [176]. During slow ramp compression, the fluid pressure in a frictionless gel becomes maximum after some pressurizing time $t_{\|}$. In our high aspect ratio $S \equiv a / h \gg 1$ compression tests on fibrin gels, however, where the network binds to the plates, this binding strongly influences its mechanical response, see Figure 3.3. Here, we propose an approximate solution to equation (3.1)-(3.3) for a disk-like bonded gel during compression, see section 3.6.1 of the the Appendix for a full derivation. This solution assumes that the shear stress induced by the friction between the gel and the rheometer plate dominates the network stress in the gel. For a given bulk modulus $K$ and shear modulus $G$, the local increase in the radial force on the gel network per unit of volume due to inhomogeneous radial strain is given by $M \partial_{r}(1 / r) \partial_{r} r U$, with $M=K+4 G / 3$ the longitudinal modulus [134], $U$ the radial displacement and $r$ the radial coordinate. The shear stress dominates the network stress when the ratio of this radial force to the local increase in radial force due to bending of the gel network $G \partial_{z}^{2} U$, with $z$ the 
vertical coordinate, is small, i.e., $M / G S^{2} \ll 1$. The solution interpolates between initial volume-conserving (VC) compression, during which no significant outflow of fluid occurs and of which the network displacement field and fluid pressure are well known [177], and pressurized compression in which the fluid pressure is maximal, see Figure 3.3C. The dominant part of the normal force $F$ is found as

$$
\frac{F}{\pi a^{2}}=T(t)\left(\frac{\eta a^{2}}{8 k} \dot{\epsilon}+M \epsilon(t)\right)+(1-T(t)) 2 G \epsilon,
$$

where $\dot{\epsilon} \equiv v / h$ is the strain rate with $v$ the velocity of the upper plate and $h$ the initial height of the gel, and $\epsilon \equiv \dot{\epsilon} t$ is the engineering strain. Equation (3.4) shows that when $T(t) \approx 1, F$ is composed of two contributions: the first stems from the compression-induced fluid pressure and the second from the normal force response of the fibrin network. The first term is proportional to the strain rate $\dot{\epsilon}$ and increases with decreasing permeability $k$ of the network, whilst the second term is proportional to the engineering strain $\epsilon$, and grows proportionally to the longitudinal modulus $M$ of the network. Equation (3.4) gives a quantitative prediction for the evolution of the normal force from the outset of compression into the pressurized phase, needed to describe the normal force during the full range of ramp compression, see the blue curve in Figure 3.1.

The transition function $T(t)$ is given by

$$
\begin{aligned}
T(t) & =1-\exp \left(-12 \frac{t}{t_{\perp}}\right), \\
t_{\perp} & =\frac{h^{2} \eta}{k G},
\end{aligned}
$$

where $t_{\perp}$ is the pressurizing time. The rate of fluid outflow increases until the fluid pressure distribution in the gel is maximal, i.e., the gel is pressurized when $T(t) \approx 1$. Once pressurized, equation (3.4) agrees with the normal force for load-controlled compression [33]. The time scale of relaxation $t_{\perp}$ does not depend on the initial radius $a$ of the gel, because the main contribution to the pressure of the fluid is induced by bending of the fibrin network, i.e., from vertical, $h$-dependent curvature in the radial displacement field. For comparison, radial gradients in the radial strain field relax on a time scale $t_{\|}=a^{2} \eta / k M=t_{\perp} S^{2} G / M \gg t_{\perp}$, implying a geometry-induced separation of time scales for relaxations of vertical and radial strain gradients.

The compression of small-pore fibrin networks suggests the network to strain-stiffen, 
see Figure 3.2, but only for small fluid pressures where the gel deforms approximately volume-conserving. We accommodate this phenomenologically by replacing $G t \rightarrow \int_{0}^{t} \mathrm{~d} t^{\prime} G\left(t^{\prime}\right)$ in the approximate solution wherever $G$ enters, with the shear modulus $G(t)$ increasing instantaneously at an onset time $t_{\mathrm{c}}$. Notwithstanding that strain-stiffening is a continuous process, this instantaneous increase is, in the absence of knowledge of the details, a minimal form to incorporate strain-stiffening, and gives

$$
G(t) \equiv G_{0}+\left(G_{\mathrm{c}}-G_{0}\right) H\left(t-t_{\mathrm{c}}\right),
$$

with $G_{0}$ the measured shear modulus of the undeformed gel, $G_{\mathrm{c}}$ the augmented shear modulus and $H(t)$ the Heaviside step function.

In simple shear experiments [178], strain-stiffening starts to occur at an onset shear stress $\sigma_{\mathrm{c}}$. In our compression experiments, we assume the onset stress to be proportional to the average tangential network stress $\bar{\sigma}_{r z}^{\prime}$ at the gel-plate interface at time $t=t_{\mathrm{c}}$

$$
\bar{\sigma}_{r z}^{\prime} \equiv \frac{1}{\pi a^{2}} \int_{0}^{a} \mathrm{~d} r 2 \pi r \sigma_{r z}^{\prime}\left(z=h, t=t_{\mathrm{c}}\right),
$$

which can be calculated using the solution for the network displacement field presented in section 3.6.1 of the Appendix, giving

$$
\sigma_{\mathrm{c}} \propto T\left(t_{\mathrm{c}}\right) \frac{\eta h a}{6 k} \dot{\epsilon} .
$$

The onset stress is a property of the fibrin network, implying its magnitude to be independent of the aspect ratio $S=a / h$ of the gel, contrary to what equation (3.9) suggests at a first glance. Below, we show, however, that $\sigma_{\mathrm{c}}$ is indeed independent of the aspect ratio.

To study the mechanical response of the fibrin networks outside of the linear regime, we performed compression tests on large-pore fibrin gels up to $80 \%$ compressive strain, $\epsilon \leq 0.8$. For these experiments, we assume a phenomenological form for the normal force in the pressurized phase, based on equation (3.4), by retaining the form of the fluid pressure term, but with a strain-dependent permeability, and by replacing the elastic contribution with the Toll model normal force response of a fibrous network under large compression [169, 179], giving

$$
\frac{F}{\pi a^{2}}=\frac{\eta a^{2}}{8 k(\epsilon)} \dot{\epsilon}+b E_{\mathrm{f}}\left(\phi^{3}(\epsilon)-\phi_{0}^{3}\right)
$$


where $E_{\mathrm{f}}$ is the Young's modulus of a single fibrin fiber, $\phi(\epsilon)=\phi_{0} /(1-\epsilon)$ is (approximately) the strain dependent volume fraction of the fibrin network with $\phi_{0}$ the volume fraction in the initial state, and $k(\epsilon)=k_{0}(1-\epsilon)$ is (approximately) the permeability of the fibrin network, with $k_{0}$ its initial value, see section 3.6.2 of the Appendix for more information. Finally, $b$ is a proportionality constant.

\subsection{Results}

To probe the capability of our theoretical framework to infer both the permeability and the elastic properties of a biopolymer network from simple compression tests, we performed compression experiments on both large-pore and small-pore fibrin fiber networks. Large-pore fibrin networks have a mesh size of about $\zeta \sim 1 \mu \mathrm{m}$, whereas small-pore networks have $\zeta \sim 0.1 \mu \mathrm{m}$ [172]. Therefore, we expect the latter to have a much smaller permeability $k \propto \zeta^{2}$ and, from equation (3.4) and (3.6), a larger

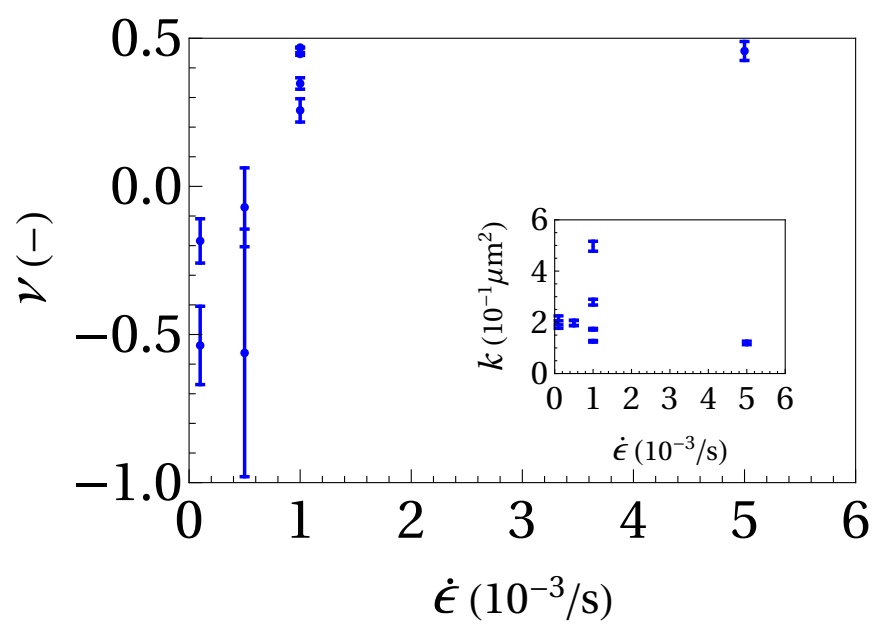

Figure 3.4: Poisson's ratio $\nu$ of large-pore fibrin gels as a function of the strain rate $\dot{\epsilon}$, inferred from ramp compression measurements. All gels were prepared at a fibrinogen concentration of $2 \mathrm{mg} / \mathrm{mL}$, were compressed up to an engineering strain of $5 \%$ or $10 \%$ and have an aspect ratio of either $S \equiv a / h=20$ or $S=10$, with $a$ the radius and $h$ the height of the gel before compression. Poisson's ratio grows with strain rate, suggesting that the fluid velocity has a marked influence on the elastic response of the fibrin network. Inset: the fitted permeability $k$ is independent of the strain rate, as expected, though it shows a large sample-to-sample variation. 
normal force in the pressurized phase and a larger pressurizing time $t_{\perp}$, which we indeed observe by comparing the maximum normal force during compression and the pressurizing time between the large-pore and small-pore experiment in, respectively, Figure 3.1 and 3.2. For details of the fitting procedure and all fit results, we refer to section 3.6.3 of the Appendix.

\subsubsection{Large-pore fibrin gels}

Comparing the measured normal force of large-pore and small-pore fibrin networks, see respectively Figure 3.1 and the inset of Figure 3.2, we observe no supralinear initial increase for large-pore fibrin, see section 3.4.2 for further elaboration on this point. Therefore, we assume the shear modulus of the large-pore samples to remain equal to $G_{0}$ throughout compression, i.e., the independently measured shear modulus just before compression, while we fit the permeability $k$ and the longitudinal modulus $M$ as free parameters. The different large-pore gel samples show a large sample-tosample variability for the fitted permeability $k$ under equal conditions, see the inset of Figure 3.4, and do not suggest dependence of the permeability on the strain rate. The compressibility of the fibrin network seems to decrease with strain rate, however, as evidenced by an increasing Poisson's ratio $\nu=\left(M-2 G_{0}\right) /\left(2 M-2 G_{0}\right)$, see Figure 3.4. As fibrin and other biopolymer networks are known to exhibit (strong) nonlinear effects, even for small strains [96-99], we consider this Poisson's ratio to be an effective value over the range of applied compressive strain. For strain rates close to zero, i.e., $\dot{\epsilon}=0.1 \cdot 10^{-3} / \mathrm{s}$, we find negative values for Poisson's ratio with a large estimation uncertainty. In earlier work [167], it was found that in the static limit fibrin networks seem to have a Poisson's ratio of zero, although no uncertainty estimation was given. If one calculates the radial extension of a static linear elastic solid bound to the plates [177], however, it is found that for negative Poisson's ratios, $-1 \leq \nu \leq 0$, the maximum radial extension is very small: it is less than $30 \mu \mathrm{m}$ for a gel with radius $a=20 \mathrm{~mm}$ and $h=1 \mathrm{~mm}$ under $10 \%$ compressive strain. As the mesh size of a large-pore fibrin network is about $\zeta \sim 1 \mu \mathrm{m}$, this radial extension is on the boundary of being meaningful in the poroelastic continuum approach we use. Therefore, we deem our finding of negative Poisson's ratio near the static limit to be consistent with literature.

To further investigate the dependence of the mechanical response of the fibrin net- 


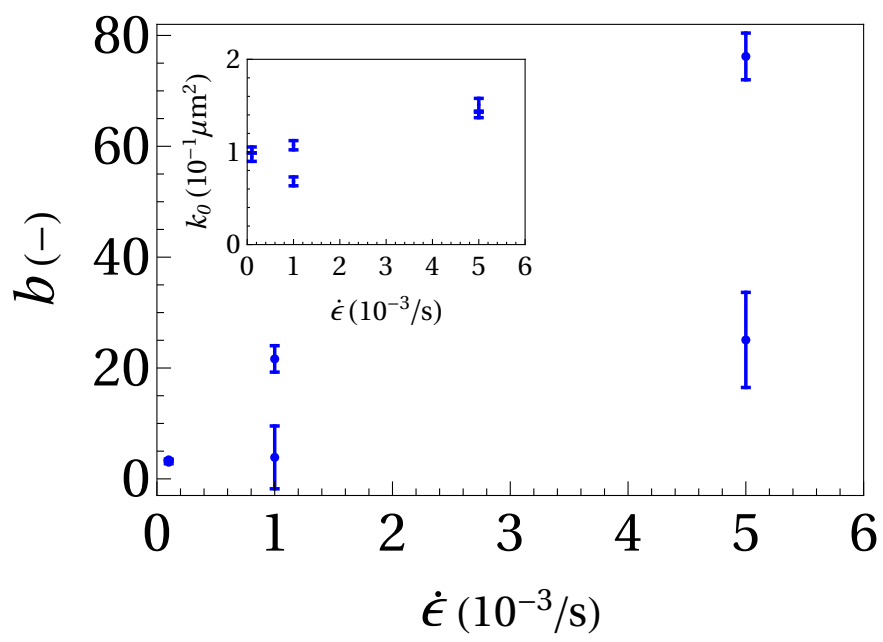

Figure 3.5: The proportionality constant $b$ of the Toll model obtained by fitting the normal force response, see equation (3.10), of a large-pore fibrin fiber network in the pressurized phase during compression experiments up to $80 \%$ strain as a function of strain rate $\dot{\epsilon}$, at a fibrinogen concentration of $2 \mathrm{mg} / \mathrm{mL}$. For increasing strain rate, $b$ grows, suggesting a higher fluid velocity to induce a stronger mechanical response of the fibrin network, similar to the increase in Poisson's ratio $\nu$ for the 5-10\% compression experiments shown in Figure 3.4. Inset: although there is a large sample-to-sample variation, see also the inset of Figure 3.4, the fitted initial permeability $k_{0}$ does not vary appreciably with strain rate, as expected.

work on the applied strain rate, we performed compression experiments of eight consecutive compression ramps of $10 \%$ engineering train, while we let the gel relax fully in between the ramps. Using equation (3.10) we find the permeability at zero strain $k_{0}$ and the proportionality constant of the Toll model [179] $b$ by fitting the maximum value of the normal force during each compression step, where the fluid pressure is assumed to be maximal, see Figure 3.5. Again, given a large sample-to-sample variability, the estimated initial permeability $k_{0}$ seems to be independent of the strain rate, as expected, see the inset of Figure 3.5. The proportionality constant $b$, however, depends significantly on the strain rate. For vanishing strain rate $\dot{\epsilon}$ it approaches a value of order unity, which agrees with literature [169]. 


\subsubsection{Small-pore fibrin gels}

Small-pore fibrin gels exhibit a qualitatively different increase in normal force during compression, see the inset of Figure 3.2, as compared to a large-pore gel, see Figure 3.1. Initially, the normal force increases as one would expect when the volume of the gel is conserved, based on the normal force of a linear elastic volume-conserving solid [177] with a shear modulus equal to that of the uncompressed fibrin network $G_{0}$, see the blue line in the inset of Figure 3.2. Afterwards, it increases supralinearly before it starts to relax due to fluid outflow, similar as with a large-pore gel. The initial supralinear increase suggests that the gel network stiffens while the fluid pressure is still low, which we take into account, as introduced in section 3.3, by assuming a stepwise increase in the shear modulus at some onset time $t_{\mathrm{c}}$, providing the red curve fit in Figure 3.2. Strain-stiffening in small-pore fibrin gels is to be expected, as their small permeability provides a relatively long volume-conserving compression, which is a shearing deformation, and fibrin networks are known to stiffen under shear [180]. Given the fitted values for the onset time $t_{\mathrm{c}}$ at which the shear modulus increases, we can calculate the onset stress $\sigma_{\mathrm{c}}$ at which stiffening occurs for the different fibrinogen concentrations we experimentally realized, see Figure 3.6. The black curve is a power law fit to the calculated onset stresses, suggesting a sharp dependence on the fibrinogen concentration of the gel.

As mentioned in section 3.3, the calculated onset stress should not depend on the aspect ratio $S$ of the gel, since it is a microscopic property of the fibrin network. In the inset of Figure 3.6, the calculated onset stress ( $)$ and the fitted onset times $(\checkmark)$ are shown for the experiments at $c=6 \mathrm{mg} / \mathrm{mL}$, where three experiments have been performed at an aspect ratio of $S=20$ (blue) and three at $S=10$ (red). It shows that the onset time at which stiffening occurs depends on the aspect ratio of the gel, but the onset stress does not. Therefore, the measure we defined for the onset stress in equation (3.9) seems appropriate. The geometry dependence of the stiffening onset time is expected, because for higher aspect ratio less compression is needed to establish a given average shear strain $\gamma_{\|} \propto \dot{\epsilon} t_{\mathrm{c}} a / h$ in the gel.

As the gel is compressed further after the onset time $t_{\mathrm{c}}$, the fluid pressure increases due to increased bending of the fibrin network, thereby increasing the average tangential stress at the sample-plate interface: a measure for the magnitude of the shear stress in the sample. Therefore, due to increased shear stress, one would expect the 
shear modulus to increase further during compression. In our model, however, we assume the shear modulus to remain constant at the augmented value, which was attained at the onset time $t_{\mathrm{c}}$. The red curve fit closely matches the measurements throughout compression, see Figure 3.2, suggesting further strain-stiffening of the fibrin network to be somehow suppressed.

The fitted permeabilities $k$ of the small-pore fibrin networks scale as $k \propto c^{n}$, with $n=-2.2 \pm 0.5$, see Figure 3.7. On the basis of a simple cubic lattice model, this result suggests that the number of protofibrils per fibrin fiber cross-section for smallpore fibrin decreases with fibrinogen concentration, see section 3.6.2 of the Appendix for more information. Finally, due to small pores the equilibrium time $t_{\perp}$ is generally larger than the compression time $t_{\mathrm{e}}$ and the fluid pressure contribution is relatively large compared to the network elasticity contribution. Therefore, and because of the uncertainty in the normal force measurements, we were unable to estimate the longitudinal modulus $M$ from normal force measurements on all small-pore gels, and consequently we cannot calculate Poisson's ratio.

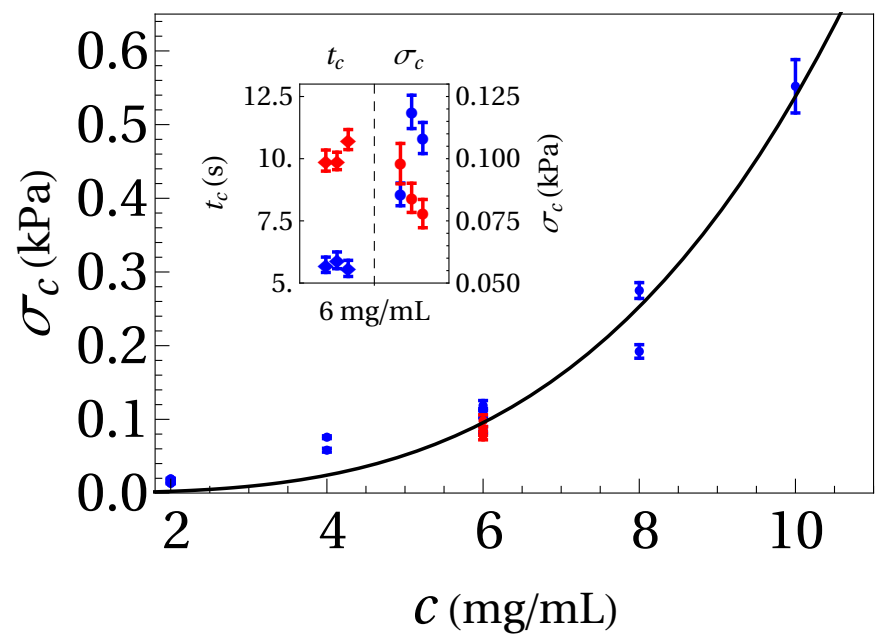

Figure 3.6: The onset stress $\sigma_{\mathrm{c}}$ at which the shear modulus of small-pore fibrin gels increases as a function of fibrinogen concentration $c$. The onset stress can be fitted with a power law $\sigma_{\mathrm{c}}=\sigma_{\mathrm{c} \text {,ref }}\left(c / c_{\mathrm{ref}}\right)^{n}$ where we choose as a reference concentration $c_{\text {ref }}=2 \mathrm{mg} / \mathrm{mL}$, and we fit $\sigma_{\mathrm{c}, \text { ref }}=2.3 \pm 0.8 \mathrm{~Pa}$ and $n=3.4 \pm 0.2$, with the estimation uncertainty in brackets. The inset shows that the stiffening onset time $t_{\mathrm{c}}(\checkmark)$ depends on aspect ratio, blue symbols correspond to $S \equiv a / h=20$ and red to $S=10$, whereas the onset stress $\sigma_{\mathrm{c}}(\mathbf{)})$ is geometry independent. 


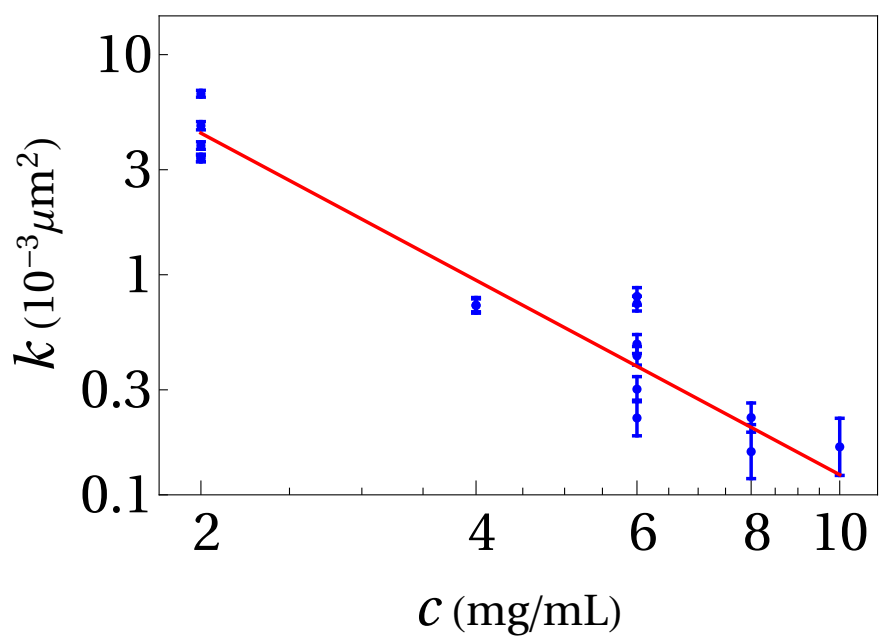

Figure 3.7: The permeability of small-pore fibrin gels as a function of the overall fibrinogen concentration $c$. The red line is a fit of $k=k_{\text {ref }}\left(c / c_{\text {ref }}\right)^{n}$, where we choose $c_{\text {ref }}=2 \mathrm{mg} / \mathrm{mL}$ as a reference concentration and fit $k_{\text {ref }}=(4.4 \pm 0.3) \cdot 10^{-3} \mu \mathrm{m}^{2}$ and $n=-2.2 \pm 0.5$, with the estimation uncertainty in brackets. This result implies that the number of protofibrils per fibrin fiber cross-section decreases with the fibrinogen concentration, see section 3.6.2 of the Appendix for more information.

\subsection{Conclusions and discussion}

We formulate a closed-form approximate solution to the poroelastic equations of motion which allows, with appropriate phenomenological extensions, to obtain the permeability and the elastic properties of a soft bonded biopolymer network from the measured time-dependent normal force in a ramp compression test. This approximate solution, appropriate for disk-like gels bonded to the rheometer plates, differs strongly from that for frictionless gels, it distinguishes the fluid and network contribution to the normal force, and it holds for all times during ramp compression, which allows for the quantification of strain stiffening during compression, in contrast to previous approximate approaches $[33,181]$.

The normal force contribution of the large-pore fibrin networks is found to increase with increasing strain rate, suggesting fluid flow through the network to make it more resistant against volume change, both for small $(5-10 \%)$ and large $(\leq 80 \%)$ compressive strains. This strain rate dependence suggests that the network-fluid interactions cause a change in the elastic response of the fibrin fiber network. The microscopic 
details giving rise to this change in the elastic response are a subject for further research.

In earlier work the normal force response of fibrin gels has been found to be dependent on the strain rate of compression $[168,169]$. In these works the contributions of fluid pressure and the fibrin network were not separated in a biphasic model, however, and the network elasticity under compression could not be extracted, see for example Figure 5 in Kim et al. [169]. Our work enables the separation of fluid pressure and network elasticity and shows that, additionally to the fluid pressure contribution, the network elasticity also depends on the applied strain rate.

The small-pore fibrin networks are found to strain-stiffen close to the start of compression, and the shear modulus is found to remain at the magnitude attained at the onset time although the shear stress on the network still increases. Possibly, the flow of fluid through the fiber network suppresses the irreversible changes in the hierarchical structure of the fibrin network which are needed for reversible strainstiffening [182].

From compression experiments on small-pore fibrin gels we find the permeability to scale inverse squared with fibrinogen concentration. With the cubic lattice model this suggests that the number of protofibrils per fibrin fiber cross-section decreases with fibrinogen concentration. Considering the complex polymerization kinetics of fibrin from its soluble precursor fibrinogen [94], this dependence could be caused by the enhanced local depletion of protofibrils with increasing fibrinogen concentration, thereby decreasing the number of protofibrils per fiber cross-section [183].

This work quantitatively describes how the complex mechanical behaviour of biopolymer systems can be decomposed into simple physical principles. It provides an alternative method to determine the hydraulic permeability of biopolymer systems based on simple compression measurements, rather than flow-through assays, with the added benefit that their elastic properties are probed at the same time. Therefore, we expect our findings to prove fruitful in, for example, mechanobiological investigations of the relation between fluid flow and the elasticity of biopolymer networks and soft tissues. 

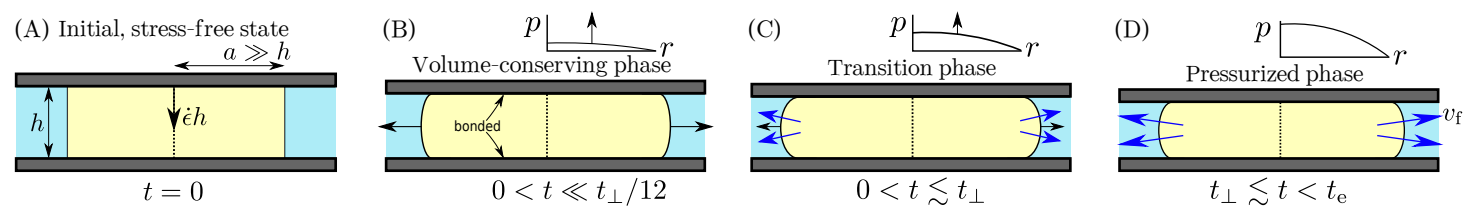

Figure 3.8: The compression phases of (A) an initially stress-free cylindrical fibrin gel (yellow) of radius $a$ and height $h$ with high aspect ratio $S \equiv a / h \gg 1$ (Figure not on scale). The gel is ramp compressed in a parallel-plate rheometer with the upper plate (gray) having a constant velocity $\dot{\epsilon} h$, where $\dot{\epsilon}$ is the strain rate. B) Compression commences with the volume-conserving phase. The fibrin network starts to bulge out (black arrows) because the gel is bonded to the plates, causing the fluid pressure $p$ to build up. $\mathbf{C}$ The build-up proceeds, causing a fluid outflow velocity $v_{\mathrm{f}}$ (blue arrows) due to the fluid pressure gradient, $v_{\mathrm{f}} \propto-\partial p / \partial r$ with $r$ the radial coordinate in the fibrin gel. After the pressurizing time $t_{\perp}$, the outward bulging of the gel network, which induces the fluid pressure, stabilizes. C) In the pressurized phase, the gel is compressed further at maximal fluid pressure until at time $t_{\mathrm{e}}$ the compression stops.

\subsection{Appendix}

In this Appendix we first present in section 3.6.1 our approximate solution to the poroelastic equations of motion, equation (1)-(3) in the manuscript. Afterwards, we treat the assumptions on which the approximate form of the poroelastic equations is based, as well as the assumptions underlying our solution. Next, we consider in section 3.6.2 the cubic lattic model used to estimate the dependence of the permeability $k$ of a fibrin network on the concentration of fibrinogen $c$ and the compressive strain $\epsilon$. In section 3.6.3, we present the fit results of the measured normal force in all compression experiments and discuss them systematically.

\subsubsection{Approximate solution}

The approximate closed-form solution to the poroelastic equations of motion is found as an exact solution to an approximate form of the poroelastic equations. First, we state this exact solution. Afterwards, we motivate the approximate form of the poroelastic equations, and detail the underlying assumptions.

We consider the ramp compression of a cylindrical gel bonded to the plates of a parallel-plate rheometer, where the gel network is treated as a linear elastic solid with 
shear modulus $G$ and bulk modulus $K$, see Figure 3.8 and Figure 2 of the manuscript. The gel network has a permeability $k$, the fluid has dynamic viscosity $\eta$, and the gel is compressed at a strain rate $\dot{\epsilon} \equiv v / h$, with $v$ the velocity of the upper plate. Before compression, the gel has an axial length $h$, a radius $a$ and a large aspect ratio $S \equiv a / h \gg 1$, see Figure 3.8A. The solution of the exact poroelastic equations, equation (1)-(3) of the manuscript, can be specified by the displacement field of the gel network $\boldsymbol{U}$, the velocity field of the fluid $\boldsymbol{v}_{\mathrm{f}}$ and the fluid pressure $p$. For notational convenience, however, we do not use the fluid velocity, but the volumeaveraged local velocity of the gel $\boldsymbol{V} \equiv \phi_{\mathrm{n}} \boldsymbol{v}_{\mathrm{n}}+\phi_{\mathrm{f}} \boldsymbol{v}_{\mathrm{f}}$ instead, with $\phi_{\mathrm{f}}\left(\phi_{\mathrm{n}}\right)$ the volume fraction and $\boldsymbol{v}_{\mathrm{f}}\left(\boldsymbol{v}_{\mathrm{n}} \equiv \partial_{t} \boldsymbol{U}\right)$ the velocity field of the fluid(gel network). In the exact solution of the approximate form of the poroelastic equations, for any radial position $r$, vertical position $z$ and time $t$ with $(r, z, t) \in] 0, a] \times[0, h] \times[0, \infty]$, the displacement field of the gel network $\boldsymbol{U}(r, z, t)=U(r, z, t) \hat{\boldsymbol{r}}+W(z, t) \hat{\boldsymbol{z}}$, with $U(r, z, t)$ the radial displacement and $W(z, t)$ the vertical displacement, reads the following

$$
\begin{aligned}
& U(r, z, t)=T(t) \dot{\epsilon} t_{\perp} \frac{r}{4} \frac{z}{h}\left(1-\frac{z}{h}\right), \\
& W(z, t)=-(1-T(t)) \epsilon z \frac{z}{h}\left(3-2 \frac{z}{h}\right)-T(t) \epsilon z+ \\
& T^{2}(t) \dot{\epsilon} t_{\perp} \frac{h}{12} \frac{M-G}{M}\left(\frac{z}{h}-3\left(\frac{z}{h}\right)^{2}+2\left(\frac{z}{h}\right)^{3}\right), \\
& T(t)=1-\exp \left(-12 \frac{t}{t_{\perp}}\right),
\end{aligned}
$$

where $t_{\perp} \equiv \eta h^{2} / G k$ is the time for the gel to become pressurized, i.e., $\partial_{t} p=0$, during compression, $\epsilon=\dot{\epsilon} t$ is the compressive strain on the gel, $M=K+4 G / 3$ is the longitudinal modulus, and $T(t)$ is the transition function which brings the gel from volume-conserving compression into the pressurized phase. The fluid pressure $p(r, z, t)$ is given by

$$
\begin{aligned}
& p(r, z, t)=T(t) G \frac{S^{2}}{4} \dot{\epsilon} t_{\perp}\left(1-\left(\frac{r}{a}\right)^{2}\right)+2(1-T(t)) G \epsilon+p_{\mathrm{ext}}- \\
& 6 \frac{z}{h}\left(1-\frac{z}{h}\right)\left[(1-T(t)) M \epsilon+\left(T^{2}(t)-T(t)\right) \frac{M-G}{12} \dot{\epsilon} t_{\perp}\right],
\end{aligned}
$$

where $p_{\text {ext }}$ is the pressure of the fluid in which the gel is immersed, and the volume- 
averaged local velocity of the gel $\boldsymbol{V}(r, z, t)$ is found as

$$
\begin{aligned}
\boldsymbol{V}(r, z, t) & =(1-T(t)) \boldsymbol{V}^{\mathrm{vc}}(r, z)+T(t) \boldsymbol{V}^{\mathrm{cp}}(r, z), \\
\boldsymbol{V}^{\mathrm{vc}}(r, z) & =3 \dot{\epsilon} r \frac{z}{h}\left(1-\frac{z}{h}\right) \hat{\boldsymbol{r}}-z \dot{\epsilon} \frac{z}{h}\left(3-2 \frac{z}{h}\right) \hat{\boldsymbol{z}}, \\
\boldsymbol{V}^{\mathrm{cp}}(r, z) & =\frac{r}{2} \dot{\epsilon} \hat{\boldsymbol{r}}-z \dot{\epsilon} \hat{\boldsymbol{z}},
\end{aligned}
$$

which shows that the volume averaged local velocity of the gel transitions between that of volume-conserving compression $\boldsymbol{V}^{\mathrm{vc}}(r, z, t)$ and that in the pressurized phase $\boldsymbol{V}^{\mathrm{cp}}(r, z, t)$ where the fluid pressure is constant in time.

Equation (3.11)-(3.17) exactly solve the following approximate set of poroelastic bulk and boundary equations of motion: equation (3.18)-(3.27). First of all, equation (3.11)-(3.14) solve the exact overall force balance, see equation (1) of the manuscript, which reads

$$
\begin{aligned}
& \frac{\partial p}{\partial r}=M \frac{\partial}{\partial r} \frac{1}{r} \frac{\partial r U}{\partial r}+(M-G) \frac{\partial^{2} W}{\partial r \partial z}+G \frac{\partial^{2} U}{\partial z^{2}}, \\
& \frac{\partial p}{\partial z}=M \frac{\partial^{2} W}{\partial z^{2}}+(M-G) \frac{1}{r} \frac{\partial}{\partial r} r \frac{\partial U}{\partial z}+G \frac{1}{r} \frac{\partial}{\partial r} r \frac{\partial W}{\partial r} .
\end{aligned}
$$

Equation (3.11)-(3.17) solve an approximate form of Darcy's law, see equation (3) in the manuscript, which has been rewritten using the definition of the volume averaged local velocity of the gel $\boldsymbol{V} \equiv \phi_{\mathrm{n}} \boldsymbol{v}_{\mathrm{n}}+\phi_{\mathrm{f}} \boldsymbol{v}_{\mathrm{f}}$

$$
\begin{gathered}
v_{\mathrm{n}, r}-V_{r}=\frac{k}{\eta} \frac{\partial p}{\partial r}, \\
\left\langle v_{\mathrm{n}, z}-V_{z}\right\rangle=\frac{k}{\eta}\left\langle\frac{\partial p}{\partial z}\right\rangle,
\end{gathered}
$$

where $\langle X\rangle \equiv(1 / h) \int_{0}^{h} \mathrm{~d} z X$ is the average of $X$ over the axial height of the gel. This form of Darcy's law, which stems from the force balance of the fluid, is approximate because it requires Darcy's law in the vertical direction to be obeyed only on average. Finally, equation (3.15)-(3.17) solve the incompressibility condition of the gel which follows from mass conservation

$$
\nabla \cdot \boldsymbol{V}=0
$$

The solution in equation (3.11)-(3.13) obeys the following essential boundary conditions. Ramp compression and the binding of the gel network to the rheometer plates 
impose

$$
\begin{aligned}
& W=0 \text { and } V_{z}=0, \text { at } z=0, \\
& W=-\epsilon h \text { and } V_{z}=-\dot{\epsilon} h, \text { at } z=h, \\
& U=0, \text { at both } z=0 \text { and } z=h .
\end{aligned}
$$

At the free boundary the solution obeys the average form of the tangential overall stress balance, and an approximate form of the radial overall stress balance combined with the permeability condition

$$
\begin{aligned}
& \left\langle\sigma_{r z}^{\prime}\right\rangle=0 \text { at } r=a, \\
& \left\langle\sigma_{r r}^{\prime}(1-T(t))-p\right\rangle=-p_{\text {ext }}, \text { at } r=a,
\end{aligned}
$$

where $\sigma^{\prime}$ is the Terzaghi effective stress of the gel network, taken to be that of a linear elastic solid.

The time-dependent normal force $F(t)$ on the plates which is generated by the gel during compression, can be calculated from the overall stress at the gel-plate interface as

$$
F(t)=-\left.\int_{0}^{a} \mathrm{~d} r 2 \pi r\left(\sigma_{z z}^{\prime}-p\right)\right|_{z=0, h}
$$

which gives with equation (3.11)-(3.14)

$$
\frac{F(t)}{\pi a^{2}}=T(t)\left[G\left(\frac{S^{2}}{8}-T(t) \frac{M-G}{12 G}\right) \dot{\epsilon} t_{\perp}+M \epsilon\right]+(1-T(t)) 2 G \epsilon .
$$

The condition for the validity of the solution presented in equation (3.11)-(3.17) is that $M / G S^{2} \ll 1$. Using this validity condition, the dominant part of equation (3.29) gives equation (4) of the manuscript.

\section{Assumptions}

To study the ramp compression of a bonded disk-like gel, we make several assumptions by comparing the bonded gel with a frictionless gel having no friction with the plates of the rheometer. Similar to a frictionless gel, we assume the bonded gel to deform in a volume-conserving manner when compression commences, see Figure $3.8 \mathrm{~B}$. Also similar to a frictionless gel, the fluid pressure is assumed to reach a 
maximal value during compression, the gel is then pressurized, see Figure 3.8D. Finally, the gel transitions in the pressurizing time from the volume-conserving to the pressurized phase in a simple mono-exponential time-dependent fashion, see Figure 3.8C.

The pressurized phase Consider a cylindrical gel under compression identical to the case we treat in the manuscript, see Figure 3.8, but instead of being bonded to the plates, it experiences no friction with the plates. In this case, the exact solution of the poroelastic equations of motion, equation (1)-(3) in the manuscript, is known [176]. During sufficiently slow ramp compression, the frictionless gel becomes pressurized, i.e., $\partial_{t} p=0$, after a pressurizing time $t_{\|}=a^{2} \eta / k M$. When pressurized, the exact solution for the displacement field, the volume-averaged velocity field and the fluid pressure $p$ of the frictionless gel reads

$$
\begin{aligned}
U(r, t) & =\nu r \epsilon+a \frac{\eta \dot{\epsilon} a^{2}}{8 k M}\left(\frac{1}{2}-\nu\right)\left((3-2 \nu) \frac{r}{a}-\left(\frac{r}{a}\right)^{3}\right), \\
W(z, t) & =-z \epsilon \\
\boldsymbol{V}(r, z) & =\frac{r}{2} \dot{\epsilon} \hat{\boldsymbol{r}}-z \dot{\epsilon} \hat{\boldsymbol{z}} \\
p(r) & =\frac{\eta \dot{\epsilon}}{2 k}\left(\frac{1}{2}-\nu\right)\left(a^{2}-r^{2}\right)+p_{\mathrm{ext}},
\end{aligned}
$$

which shows that the gel deformation differs from that of a static frictionless solid by an inhomogeneous radial strain proportional to the compression rate, see equation (3.30). This inhomogeneous strain causes stress in the gel network, which in turn sources the fluid pressure $p$ required for the outflow of fluid from the gel, see equation (3.18) and (3.19). The fact that this fluid pressure is constant in time, implies a constant outflow of fluid from the gel, which brings us to the first assumption.

Assumption 1: similar to a frictionless gel, a bonded gel becomes pressurized, i.e., $\partial_{t} p=0$, after some pressurizing time $t_{\perp}$, see Figure 3.8D.

Given this assumption, we can write down the following approximate solution for a bonded gel in the pressurized phase with constant pressure, which obeys the exact 
form of equation (3.18)-(3.25)

$$
\begin{aligned}
U^{\mathrm{cp}}(r, z) & =\dot{\epsilon} t_{\perp} \frac{r}{4} \frac{z}{h}\left(1-\frac{z}{h}\right), \\
W^{\mathrm{cp}}(z, t) & =-\epsilon z+\dot{\epsilon} t_{\perp} \frac{h}{4} \frac{1}{6(1-\nu)}\left(\frac{z}{h}-3\left(\frac{z}{h}\right)^{2}+2\left(\frac{z}{h}\right)^{3}\right), \\
\boldsymbol{V}^{\mathrm{cp}}(r, z) & =\frac{r}{2} \dot{\epsilon} \hat{\boldsymbol{r}}-z \dot{\epsilon} \hat{\boldsymbol{z}}, \\
p(r) & =G \frac{S^{2}}{4} \dot{\epsilon} t_{\perp}\left(1-\left(\frac{r}{a}\right)^{2}\right)+p_{\text {ext }},
\end{aligned}
$$

where we note that in the bonded case the vertical strain is inhomogeneous instead of the radial strain in the frictionless case, and we used the permeability condition $p=p_{\text {ext }}$ at the free boundary $r=a$. The volume-averaged gel velocity field $\boldsymbol{V}^{\text {cp }}$ equals that of a frictionless volume-conserving solid because $\partial_{t} U=0$, implying all radial motion is due to fluid flow. The velocity field of the fluid equals that of a frictionless volume-conserving solid, because it is not bonded to the rheometer plates and flows through the radially static, but vertically comoving, gel network.

To study whether this approximate solution is reasonable, we consider the exact form of the bulk equations of motion, equation (3.18)-(3.22), giving

$$
\begin{aligned}
\frac{\eta}{k}\left(\frac{\partial U}{\partial t}-V_{r}\right) & =M \frac{\partial}{\partial r} \frac{1}{r} \frac{\partial r U}{\partial r}+(M-G) \frac{\partial^{2} W}{\partial r \partial z}+G \frac{\partial^{2} U}{\partial z^{2}} \\
\frac{\eta}{k}\left(\frac{\partial W}{\partial t}-V_{z}\right) & =M \frac{\partial^{2} W}{\partial z^{2}}+(M-G) \frac{1}{r} \frac{\partial}{\partial r} r \frac{\partial U}{\partial z}+G \frac{1}{r} \frac{\partial}{\partial r} r \frac{\partial W}{\partial r} \\
\nabla \cdot \boldsymbol{V} & =0
\end{aligned}
$$

where we eliminated the fluid pressure $p$. The boundary conditions (BCs) are equations (3.23)-(3.25), combined with the exact boundary conditions at the free boundary: the overall force balance and the permeability condition

$$
\begin{aligned}
\sigma_{r r}^{\prime}-p & =-p_{\text {ext }}, \text { at } r=a, \\
\sigma_{r z}^{\prime} & =0, \text { at } r=a, \\
p & =p_{\text {ext }}, \text { at } r=a,
\end{aligned}
$$

where equation (3.41) is the overall balance of forces perpendicular to the free boundary, equation (3.42) is the overall balance of forces tangential to the free boundary, and equation (3.43) expresses that the gel network is permeable for fluid. 
The exact solution in the constant pressure phase can be written as $\boldsymbol{U}=\boldsymbol{U}^{\mathrm{cp}}+\Delta \boldsymbol{U}$ and $\boldsymbol{V}=\boldsymbol{V}^{\mathrm{cp}}+\Delta \boldsymbol{V}$, with $\Delta \boldsymbol{U}$ and $\Delta \boldsymbol{V}$ the difference solution, i.e., the difference between the exact and the approximate solution in the pressurized phase. To show that the difference may be negligible, we scale all quantities to their presumed typical sizes: $\tilde{U}=\Delta U / h, \tilde{W}=\Delta W / h, \tilde{V}_{r}=\Delta V_{r} /\left(a / t_{\text {ext }}\right), \tilde{V}_{z}=\Delta V_{z} /\left(h / t_{\text {ext }}\right), \xi=$ $z / h, \rho=r / a, \tau_{\text {ext }}=t / t_{\text {ext }}$ and we define $t_{\text {ext }}$ as the externally determined time scale of ramp compression, $t_{\mathrm{ext}}=h / v$ with $v$ the velocity of the upper plate. The bulk equations of motion for the difference solution read

$$
\begin{aligned}
\frac{\partial \tilde{U}}{\partial \tau_{\mathrm{ext}}}-S \tilde{V}_{r} & =\frac{t_{\mathrm{ext}}}{t_{\perp}}\left(\frac{M}{G S^{2}} \frac{\partial}{\partial \rho} \frac{1}{\rho} \frac{\partial \rho \tilde{U}}{\partial \rho}+\frac{M-G}{G S} \frac{\partial^{2} \tilde{W}}{\partial \rho \partial \xi}+\frac{\partial^{2} \tilde{U}}{\partial \xi^{2}}\right) \\
\frac{\partial \tilde{W}}{\partial \tau_{\mathrm{ext}}}-\tilde{V}_{z} & =\frac{t_{\mathrm{ext}} M}{t_{\perp} G}\left(\frac{\partial^{2} \tilde{W}}{\partial \xi^{2}}+\frac{1-\frac{G}{M}}{S} \frac{1}{\rho} \frac{\partial}{\partial \rho} \rho \frac{\partial \tilde{U}}{\partial \xi}+\frac{G}{M S^{2}} \frac{1}{\rho} \frac{\partial}{\partial \rho} \rho \frac{\partial \tilde{W}}{\partial \rho}\right) \\
\tilde{\nabla} \cdot \tilde{\boldsymbol{V}} & =0
\end{aligned}
$$

where $t_{\perp}=h^{2} \eta / k G$ is the pressurizing time, and the boundary conditions read

$$
\begin{gathered}
\tilde{W}=0, \tilde{V}_{z}=0 \text { and } \tilde{U}=0, \text { at both } \xi=0 \text { and } \xi=1 \\
M \frac{\partial \tilde{U}}{\partial \rho}+\Lambda \frac{\tilde{U}}{\rho}=-S\left(\Lambda \frac{\partial \tilde{W}}{\partial \xi}+\Lambda\left\{-\epsilon+\frac{\dot{\epsilon} t_{\perp}}{24(1-\nu)}\left[1-6\left(\frac{z}{h}-\left(\frac{z}{h}\right)^{2}\right)\right]\right\}+\right. \\
\left.(M+\Lambda) \frac{\dot{\epsilon} t_{\perp}}{4}\left\{\frac{z}{h}-\left(\frac{z}{h}\right)^{2}\right\}\right), \text { at } \rho=1 \\
\frac{\partial \tilde{W}}{\partial \rho}=-S\left(\frac{\partial \tilde{U}}{\partial \xi}+\dot{\epsilon} t_{\perp} \frac{r}{4 h}\left\{1-2 \frac{z}{h}\right\}\right), \text { at } \rho=1
\end{gathered}
$$

If we assume that $M / G S^{2} \ll 1$, then the $M \partial_{r}(1 / r) \partial_{r} r U$ term is much smaller than the $G \partial_{z}^{2} U$ term, compare equation (3.38) to equation (3.44), and the $G(1 / r) \partial_{r} r \partial_{r} W$ term is much smaller than the $M \partial_{z}^{2} W$ term since $G<M$, compare equation (3.39) to equation (3.45). Neglecting the small terms, both equation (3.44) and (3.45) are only first order in $r$ instead of second order. The boundary conditions for $U$ and $W$ at $r=0$ are imposed by smoothness and symmetry: $U=0$ and $\sigma_{r z}=0 \Longrightarrow \partial_{r} W=$ 0 at $r=0$, and can not be ignored. Therefore, we must neglect the Robin boundary condition for $U$ at $r=a$ in equation (3.48), and the Neumann boundary condition 
for $W$ at $r=a$ in equation (3.49). Then, it follows that there are no sources for a nontrivial solution to equation (3.44)-(3.47), implying that $\tilde{U}=0$ and $\tilde{\boldsymbol{V}}=0$ is the solution. Therefore, the approximate solution in equation (3.34)-(3.36) is expected to be accurate in the pressurized phase, provided that $M / G S^{2} \ll 1$.

The neglect of the two small terms is equivalent to treating the dependencies of $\tilde{U}$ and $\tilde{\boldsymbol{V}}$ to the radial coordinate $r$ as static. In fact, the relative size of the different network stress terms, provided $M / G S^{2} \ll 1$, holds during the whole of compression, which gives the full time-dependent approximate solution, contained in equation (3.11),(3.12) and (3.15)-(3.17) a static dependence on $r: U(r, z, t) \propto r^{1}$, $W(z, t) \propto r^{0}, V_{r}(r, z, t) \propto r^{1}$ and $V_{z}(z, t) \propto r^{0}$ for all times $t$.

Physically, the condition for the approximate solution to hold, $M / G S^{2} \ll 1$, means that the radial force in the gel network due to bending of the gel network, sourced by $G \partial_{z}^{2} U$, is much greater than the radial force due to inhomogeneity in the radial strain, sourced by $M \partial_{r}(1 / r) \partial_{r} r U$. That is, the shear stress induced by friction between the gel and the rheometer plate dominates the network stress in the gel. Similarly, the vertical force in the gel network due to inhomogeneity in the vertical strain, sourced by $M \partial_{z}^{2} W$, is much greater than the vertical force induced by vertical out of plane displacements, sourced by $G(1 / r) \partial_{r} r \partial_{r} W$. The term $(M-G) \partial_{z} \partial_{r} W$ can be smaller or comparable to the $G \partial_{z}^{2} U$ term, depending on the size of the aspect ratio $S \gg 1$, likewise for the size of $(M-G)(1 / r) \partial_{r} r \partial_{z} U$ relative to $M \partial_{z} W$. Finally, it should be noted that although the presumed magnitude of $U$, which we take to be $h$, is uncertain, this presumption does not influence the condition to neglect the radial inhomogeneity term, because the shear stress term, relative to which the radial inhomogeneity term is small, also scales with $U$.

The volume-conserving phase Next, we consider that in the frictionless case [176] for short times after the commencement of compression, i.e., $\pi^{2} t / t_{\|} \ll 1$ with $t_{\|}$the pressurizing time, the gel deforms to good approximation as a volume-conserving frictionless solid, because the outflow of fluid from the gel network is still small. Again, assuming the bonded case to behave analogous to the frictionless case, we arrive at the second assumption.

Assumption 2: similar to a frictionless gel, for times much shorter than the, yet to be determined, pressurizing time $t_{\perp}$, a bonded gel deforms like a bonded volume- 
conserving solid, see Figure 3.8B.

The (quasi)-static displacement field of a volume-conserving linear elastic solid bonded to the plates is [177]

$$
\begin{aligned}
U^{\mathrm{vc}}(r, z, t) & =3 \epsilon r \frac{z}{h}\left(1-\frac{z}{h}\right), \\
W^{\mathrm{vc}}(z, t) & =-z \epsilon \frac{z}{h}\left(3-2 \frac{z}{h}\right),
\end{aligned}
$$

which gives equation (3.18) and (3.19) in dimensionless form as

$$
\begin{aligned}
& \frac{\partial \tilde{p}}{\partial \rho}=-6 S^{2} \epsilon \rho, \\
& \frac{\partial \tilde{p}}{\partial \xi}=-6 \epsilon(1-2 \xi),
\end{aligned}
$$

where we defined $\tilde{p}=p / G$. As $S \gg 1$, the majority of the pressure builds up radially, stemming from the $G \partial_{z}^{2} U^{\mathrm{vc}}$ term in equation (3.18). This term quantifies the stress induced by bending of the gel network, which is imposed by the binding of the network to the plates. At $z=0$ and $z=h$, we find that $\partial p / \partial z \neq 0$, however, which implies with Darcy's law, see equation (3) of the manuscript, a vertical flow of fluid relative to the gel network through the impermeable plates of the rheometer. As this would render the plates permeable, we adopt an approximative form of the vertical part of Darcy's law, see equation (3.21), by requiring it to hold only when averaged over the axial height of the gel.

Transition from the volume-conserving phase to the pressurized phase The frictionless gel transitions from volume-conserving compression to the pressurized phase on a pressurizing time scale $t_{\|}[176]$. Also, previous work in the context of gel compression demonstrated mono-exponential time-dependence, see equation (32) in Yamaue et al. [181]. This brings us to the final assumption

Assumption 3: we assume the time-dependent dynamics of the radial displacement of the gel network, transitioning from volume-conserving deformation to the pressurized phase, to be proportional to a transition function $T(t)$, see Figure 3.8C.

We write $U(r, z, t)=T(t) U^{\mathrm{cp}}$, such that $T(t) \approx 1$ if $t \gtrsim t_{\perp}$ with $t_{\perp}$ the pressurizing time, and $T(0)=0$, because at $t=0$ we assume the gel to be stressfree. Then, we assume that $\boldsymbol{V}(r, z, t)$, see equation (3.15), interpolates between 
the volume-conserving velocity field of a bonded solid $\boldsymbol{V}^{\mathrm{vc}}(r, z, t)$, see the timederivative of equation (3.50) and (3.51), and the volume-conserving velocity field of a frictionless solid $\boldsymbol{V}^{\mathrm{cp}}(r, z, t)$, see equation (3.36). The exact radial force balance, equation (3.18) combined with equation (3.20), can than be solved for $T(t)$, giving the transition function $T(t)$, see equation (3.13), along with the pressurizing time $t_{\perp}=h^{2} \eta / k G$. Then, by requiring also $W(z, t)$ and $p(r, z, t)$ to transition from their volume-conserving form to their form in the pressurized phase, using only $T(t)$, we obtain as a solution to equation (3.18)-(3.25) the solution contained in equation (3.11)-(3.17), but slightly more general with

$$
\begin{aligned}
W(z, t) & =-(1-T(t)) \epsilon z \frac{z}{h}\left(3-2 \frac{z}{h}\right)-T(t) \epsilon z+ \\
& T^{n}(t) \dot{\epsilon} t_{\perp} \frac{h}{12} \frac{M-G}{M}\left(\frac{z}{h}-3\left(\frac{z}{h}\right)^{2}+2\left(\frac{z}{h}\right)^{3}\right), \\
p(r, z, t) & =T(t) G \frac{S^{2}}{4} \dot{\epsilon} t_{\perp}\left(1-\left(\frac{r}{a}\right)^{2}\right)+C- \\
6 & \frac{z}{h}\left(1-\frac{z}{h}\right)\left[(1-T(t)) M \epsilon+\left(T^{n}(t)-T(t)\right) \frac{M-G}{12} \dot{\epsilon} t_{\perp}\right],
\end{aligned}
$$

where $n \geq 2$, and $C$ is an integration constant. The non-uniqueness of this solution, illustrated by the arbitrariness of $n$, may be related to the approximate nature of the equations of motion it solves.

The terms proportional to $T^{n}(t)$ are related to the inhomogeneous vertical strain in the pressurized phase, compare equation (3.35) to equation (3.54). This inhomogeneous vertical strain balances the $(M-G)(1 / r) \partial_{r} r \partial_{z} U$ term in equation (3.39), which measures the local increase in vertical force on the gel network due to the radial change in the deflection of the gel network. If we consider the vertical force balance with the exact form of Darcy's law, and plug in equation (3.11), (3.15)(3.17), and (3.54), we find that the left hand side can be grouped in terms proportional to $T(t)^{0}, T(t)^{1}, T(t)^{n-1}$ and $T(t)^{n}$, whereas the right hand side can be grouped in terms proportional to $T(t)^{0}, T(t)^{1}$ and $T(t)^{n}$. We then set the value of $n \geq 2$ by requiring the dependencies on the transition function $T(t)$ on both sides of the exact equation to match: for each term on the left hand side proportional to $T(t)^{m}$, there should be a term on the right hand side also proportional to $T(t)^{m}$. This requirement uniquely sets $n=2$.

Finally, to determine the integration constant $C$ for the fluid pressure $p$, we consider 
the force balance for the overall radial stress at the free boundary. The volumeconserving solution, see equation (3.50)-(3.53), can satisfy the weak form of the radial force balance $\left\langle\sigma_{r r}^{\prime}-p\right\rangle=-p_{\text {ext }}$ at $r=a$. On the other hand, our approximate solution, as explained above, ignores the exact boundary conditions at the free boundary in the pressurized phase, see equation (3.41)-(3.43), and thus does not obey the stress-free condition on the gel network $\sigma_{r r}^{\prime}=0$ at $r=a$, which is implied by the exact boundary conditions. This artifact of our approximate solution comes from ignoring the $M \partial_{r}(1 / r) \partial_{r} r U$ term, implying we do not take into account the relaxations in the radial strain which are induced by $\sigma_{r r}^{\prime}=0$ and which are expected to occur, similar to the frictionless case, on a time scale $t_{\|}$. Therefore, we assume an approximate condition at the free boundary which interpolates between the weak form of the overall radial force balance in the volume-conserving phase and the weak form of the permeability condition, see equation (3.43), in the pressurized phase, giving equation (3.27). This approximate condition makes the contribution of the gel network to the radial stress vanish in the pressurized phase, thereby enforcing the weak form of the permeability condition in the pressurized phase. Using equation (3.27), we then find $C=2(1-T(t)) G \epsilon+p_{\text {ext }}$. Similarly, we assume the force balance of the overall tangential stress at the free boundary, see equation (3.42), to hold in weak form, i.e., $\left\langle\sigma_{r z}^{\prime}\right\rangle=0$ at $r=a$, which is satisfied trivially due to symmetry.

\subsubsection{Cubic lattice model}

During the polymerization of a fibrin fiber network fibrinopeptides are cleaved from fibrinogen molecules, thereby creating fibrin monomers. These monomers polymerize into protofibrils which in turn form fibrin fibers by both end-to-end and lateral aggregation, see section 1.3.1 for more information. To estimate the scaling of the permeability of a fibrin network with both the pre-polymerization fibrinogen concentration and large compressive strains on the network, we model the fibrin fiber network after polymerization as a cubic lattice.

Mass conservation Consider a volume $V$ in which a fibrinogen solution with overall mass density $c$ has been polymerized into a large number of cubic cells, forming a cubic lattice. Because of the large number of cells, the volume $V$ can have any macroscopic shape. The cube edges have a length $\zeta$, the mesh size, and consist of 
Table 3.1: Experimental conditions and fit results for compression experiments on largepore fibrin gels at small strain. From the left to the right, the columns provide: the number tag given to each experiment, the concentration of fibrinogen $c$, the initial radius $a$ of the gel before compression, the strain rate at which the gel is compressed $\dot{\epsilon}$, the amount of engineering strain put on the gel after compression $\epsilon_{\mathrm{e}}$, the fitted permeability $k$, the fitted longitudinal modulus $M$, the measured shear modulus just before compression $G_{0}$ from small-strain rheometry, the calculated Poisson's ratio $\nu=\left(M-2 G_{0}\right) /\left(2 M-2 G_{0}\right)$, the exponential relaxation time $t_{\perp} / 12$ with $t_{\perp}=h^{2} \eta / k G$ the pressurizing time, and the validity condition $M / G_{0} S^{2}$. All gels had an initial height of $h=1 \mathrm{~mm}$ and the estimation uncertainty is in brackets.

\begin{tabular}{c|cccccccccc}
$\#$ & $c(\mathrm{mg} / \mathrm{mL})$ & $a(\mathrm{~mm})$ & $\dot{\epsilon}\left(10^{-3} / \mathrm{s}\right)$ & $\epsilon_{\mathrm{e}}(\%)$ & $k\left(10^{-1} \mu \mathrm{m}^{2}\right)$ & $M(\mathrm{kPa})$ & $G_{0}(\mathrm{kPa})$ & $\nu(-)$ & $t_{\perp} / 12(\mathrm{~s})$ & $M / G S^{2}$ \\
\hline \hline 1 & 2 & 20 & 1.0 & 10 & $1.26(0.03)$ & $1.45(0.12)$ & 0.139 & $0.447(0.005)$ & 3.3 & 0.026 \\
2 & 2 & 20 & 1.0 & 10 & $4.97(0.19)$ & $0.43(0.05)$ & 0.142 & $0.257(0.039)$ & 0.8 & 0.008 \\
3 & 2 & 20 & 1.0 & 10 & $2.79(0.11)$ & $0.91(0.09)$ & 0.212 & $0.347(0.019)$ & 1.0 & 0.011 \\
4 & 2 & 20 & 1.0 & 10 & $1.73(0.03)$ & $1.48(0.07)$ & 0.087 & $0.469(0.002)$ & 3.8 & 0.042 \\
5 & 2 & 10 & 1.0 & 10 & $1.54(0.02)$ & $0.15(0.01)$ & 0.227 & $1.950(0.235)$ & 1.6 & 0.007 \\
6 & 2 & 20 & 1.0 & 5 & $5.00(0.33)$ & $0.99(0.16)$ & 0.119 & $0.431(0.013)$ & 1.0 & 0.021 \\
7 & 2 & 20 & 1.0 & 5 & $2.29(0.11)$ & $1.28(0.26)$ & 0.213 & $0.401(0.024)$ & 1.2 & 0.015 \\
8 & 4 & 20 & 1.0 & 10 & $2.17(0.08)$ & $1.14(0.10)$ & 0.444 & $0.181(0.048)$ & 0.6 & 0.006 \\
9 & 4 & 20 & 1.0 & 10 & $0.63(0.02)$ & $3.43(0.34)$ & 1.400 & $0.156(0.058)$ & 0.7 & 0.006 \\
10 & 2 & 20 & 5.0 & 10 & $1.20(0.07)$ & $2.04(1.40)$ & 0.162 & $0.457(0.032)$ & 3.0 & 0.032 \\
11 & 2 & 20 & 5.0 & 10 & $1.05(0.15)$ & $0(4.10)$ & 0.139 & - & 3.9 & - \\
12 & 2 & 10 & 0.5 & 5 & $1.98(0.10)$ & $0.30(0.04)$ & 0.202 & $-0.562(0.418)$ & 1.4 & 0.015 \\
13 & 2 & 10 & 0.5 & 5 & $1.97(0.10)$ & $0.36(0.04)$ & 0.194 & $-0.071(0.133)$ & 1.5 & 0.019 \\
14 & 2 & 20 & 0.1 & 10 & $2.16(0.10)$ & $0.27(0.01)$ & 0.158 & $-0.184(0.075)$ & 1.7 & 0.004 \\
15 & 2 & 20 & 0.1 & 10 & $1.83(0.07)$ & $0.30(0.01)$ & 0.202 & $-0.537(0.132)$ & 1.5 & 0.004 \\
\hline \hline
\end{tabular}

cylindrical fibrin fibers with radius $R$ and fibrin monomer mass density $\rho$, see Figure 3.9. The total mass $m$ of fibrinogen in the volume is $m=c V$. By mass conservation, the fibrinogen mass must be equal to the mass of fibrin monomers in the fibrin fibers, implying $m=\rho V_{\text {fiber }}$ with $V_{\text {fiber }}$ the total volume of fibrin fibers, where we assume all fibrin monomers to be polymerized. As the mass of a fibrinogen molecule is about $340 \mathrm{kDa}$ and the mass of the cleaved fibrinopeptides is about $1.5 \mathrm{kDa}$, we ignore the mass difference between a fibrinogen molecule and a fibrin monomer. Assuming the mesh size to be much larger than the radius $R$ of the fibrin fibers, i.e., $\zeta / R \gg 1$, the total volume of fibrin fiber can be expressed approximately as $V_{\text {fiber }}=L \pi R^{2}$, with $L$ the total axial length of fibrin fiber in the volume. We then obtain

$$
c V=\rho L \pi R^{2}
$$

where $V=\zeta^{3} N$ and $L=3 \zeta N$, since the unit cell of a cubic lattice contains three edges of a cube. We consider equation (3.56) as an implicit function for $\zeta$ as a 


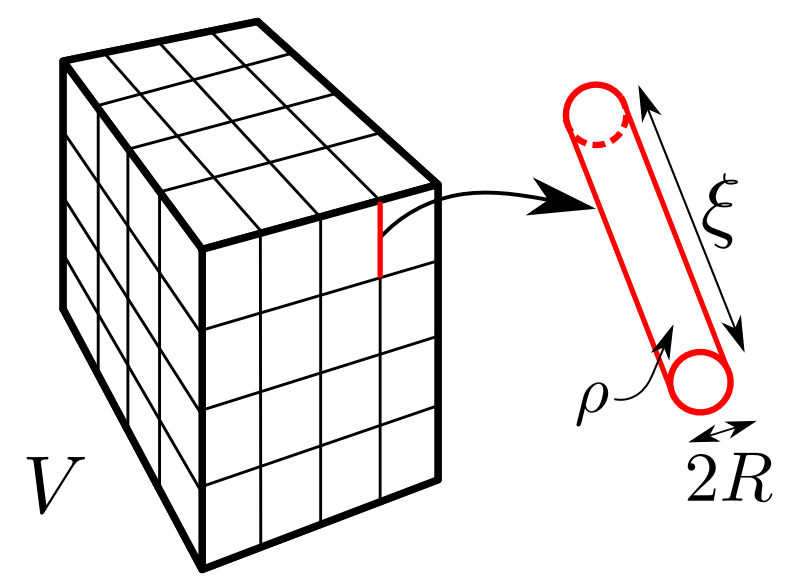

Figure 3.9: In the cubic lattice model a fibrin network of volume $V$ is modelled as a cubic lattice of fibrin fibers, where the edge length equals the mesh size $\zeta$. The fibrin fibers have a radius $R$ and mass density $\rho$.

function of $c$, so a given overall fibrinogen mass density $c$ results in a mesh size $\zeta$ after polymerization of the fibrinogen.

Scaling laws If the fibrinogen mass per unit of axial length $\pi R^{2} \rho$ depends on the fibrinogen concentration as $\pi R^{2} \rho \propto c^{n}$, we obtain $\zeta \propto c^{(n-1) / 2}$. And because the permeability is expected to scale as $k \propto \zeta^{2}$, we find $k \propto c^{n-1}$. As reported in the manuscript, we find from experiments on small-pore fibrin gels that $k \propto c^{m}$, with $m=-2.2(0.5)$ and the uncertainty in brackets. This result implies that the fibrinogen concentration per unit of axial fiber length $\pi R^{2} \rho$ decreases with the overall fibrinogen concentration $c$. suggesting the polymerization kinetics to depend on c.

As the fibrin fibers are formed out of protofibrils, which in turn consist of fibrin monomers, one can express the mass of fibrinogen in the fibrin fibers as $N m_{\text {proto }}$, with $N$ the total number of protofibrils and $m_{\text {proto }}$ the mass per protofibril. The protofibrils are formed by both end-to-end and lateral aggregation of fibrin monomers, see Figure 1.4. Therefore, the total number of protofibrils in the fibrin fibers can be calculated as $N=N_{\text {cross }} L / l_{\text {proto }}$, with $N_{\text {cross }}$ the number of protofibrils per fibrin fiber cross-section, $L$ the total axial length of fibrin fiber and $l_{\text {proto }}$ the 
length of a protofibril. The mass balance then becomes

$$
c V=\frac{L N_{\text {cross }} m_{\text {proto }}}{l_{\text {proto }}} .
$$

As the mass and length of a protofibril are both expected to be proportional to the number of fibrinogen monomers in the protofibril, we expect the ratio $m_{\text {proto }} / l_{\text {proto }}$ to be independent of the fibrinogen concentration $c$. Assuming the number of protofibrils per fiber cross-section to scale with fibrinogen concentration $N_{\text {cross }} \propto c^{d}$, we find that $k \propto \zeta^{2} \propto c^{d-1}$. Our finding that $k \propto c^{m}$, with $m=-2.2(0.5)$, thus implies that the number of protofibrils per fibrin fiber cross-section decreases with increasing fibrinogen concentration.

Large compression When a fibrin gel is under large compression, the vertically oriented fibers in the cubic lattic model will buckle. Assuming approximate homogeneous deformation, the vertical height of the buckled fibers is $\zeta(1-\epsilon)$, where $\epsilon$ is the engineering strain. As fluid flows out of the gel radially, and since the permeability $k$ is proportional to the surface area of the pores, we expect the permeability to scale as $k \propto \zeta^{2}(1-\epsilon)$, giving the compression dependent permeability as $k(\epsilon)=k_{0}(1-\epsilon)$ with $k_{0}$ the permeability at zero strain. This strain-dependent permeability we use in equation (10) of the manuscript.

\subsubsection{Fit results}

In section 3.6.1 the normal force exerted by a bonded disk-like gel under ramp compression was obtained from an approximate closed-form solution of the poroelastic equations of motion. Here, we analyze the compression experiments we performed on fibrin gels. By varying the experimental conditions, i.e., the amount of strain $\epsilon_{\mathrm{e}}$, the fibrinogen concentration $c$, the aspect ratio $S=a / h$ with the initial height $h$ and radius $a$ of the gel, and the strain rate $\dot{\epsilon}$, the microscopic response of the fibrin network will be reflected in the fitted elastic constants and the permeability. We use the Mathematica function NonlinearModelFit for fitting.

We conducted experiments at body temperature on fibrin gels having either a largepore fibrin network or a small-pore network. The large-pore gels have a typical mesh size $\zeta$ of about 1 micrometer $[172,173]$. The small-pore gels, on the other hand, 
have a mesh size of about a hundred nanometers. For the large-pore fibrin gels, compression experiments with both small and large strain have been conducted. In the small strain experiments the engineering strain, i.e., the ratio of the change in gap size over the gap size at polymerization, was maximally $10 \%$. In the large strain experiments the fibrin gels were compressed up to $80 \%$ engineering strain in subsequent steps of $10 \%$ compression. For small-pore fibrin gels only small strain compression experiments up to $10 \%$ compression were conducted.

\section{Compression of large-pore fibrin gels}

We conducted ramp compression experiments on large-pore fibrin gels under variable conditions in which we measured the normal force $F$ exerted by the gel on the rheometer as a function of time $t$. See Figure 3.10 for an example of a compression experiment with relatively high strain rate and Figure 3.11 for a compression experiment with relatively low strain rate. These experiments were conducted with an initial gap size of $h=1 \mathrm{~mm}$, and the standard conditions (SC) were chosen to be $\epsilon_{\mathrm{e}}=0.1, \dot{\epsilon}=10^{-3} / \mathrm{s}, c=2 \mathrm{mg} / \mathrm{mL}$ and $S=20$. By varying one of these conditions relative to the experiments at standard conditions, the influence of the different conditions could be studied. After compression, the gel relaxes while the engineering strain is held constant. During and after compression the normal force exerted by the gel on the rheometer is measured. Moreover, just before the start of compression, the shear modulus $G_{0}$ of the gel is measured from small oscillation rheometry.

Comparing equation (3.29) to the measured normal force, we fit the permeability $k$ and the longitudinal modulus $M=K+4 G_{0} / 3$. Table 3.1 gives the results for the fitted material parameters, the experimental conditions, and the exponential relaxation time. Moreover, we calculated $M / G_{0} S^{2}$ which, as shown in section 3.6.1, should be much smaller than unity to validly apply the approximate solution. Indeed, this is the case for all large-pore fibrin compression experiments.

From the measured shear modulus $G_{0}$ and the fitted longitudinal modulus $M$ follows the Poisson's ratio of the gel network $\nu=\left(M-2 G_{0}\right) /\left(2 M-2 G_{0}\right)$, where we assumed the ratio of the standard deviation in the measurement of the shear modulus to its measured value to be $1 \%$. The uncertainties in $G_{0}$ and $M$ are assumed to be independent because $M$ was fitted against normal force measurements, while $G_{0}$ was inferred from a torsion measurement. 


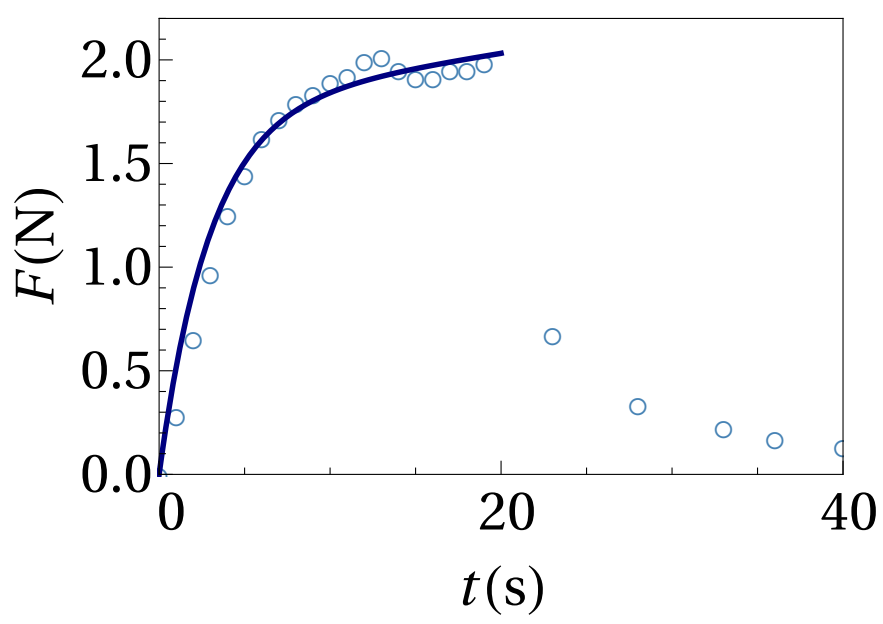

Figure 3.10: The measured normal force $F$ (blue circles) of a large-pore fibrin gel in response to fast ramp compression, see experiment 10 in Table 3.1 for the experimental conditions. The pressurizing time for the fluid pressure to build up to its maximal value is $t_{\perp}=36 \mathrm{~s}$. The blue curve is a fit of equation (3.29) to the measured normal force during compression, giving the permeability of the fibrin network $k$ and its longitudinal modulus $M$ as listed in Table 3.1.

Next, we consider the influence of the different experimental conditions on the results for the permeability $k$ and the Poisson's ratio $\nu$.

Compressive strain The standard amount of engineering strain after compression of the gel is $10 \%$. To probe the influence of strain, two compression experiments have been conducted with 5\% strain instead, experiment 6 and 7. The standard condition (SC) experiments, experiment $1 \mathrm{t} / \mathrm{m} \mathrm{4}$, give an average of the best estimates for the permeability of $\bar{k}_{\mathrm{SC}}=0.27(0.17) \mu \mathrm{m}^{2}$, with the standard deviation of the four best estimates in brackets. The uncertainty in the best estimate for each of the four individual experiments is smaller than 5\%, see Table 3.1. Moreover, the average of the Poisson's ratio best estimates of the SC experiments is $\bar{\nu}_{\mathrm{SC}}=0.38(0.10)$, with individual uncertainty all less than $15 \%$. We have four experiments at standard conditions, which is the highest number of repeated experiments within a single set of conditions in our data set. Since the individual uncertainties are much smaller than the standard deviation of the best estimates, we assume the standard deviation of the permeabilities and the Poisson's ratios to reflect the sample-to-sample variation among the different fibrin gels [162]. The variation probably arises from the 


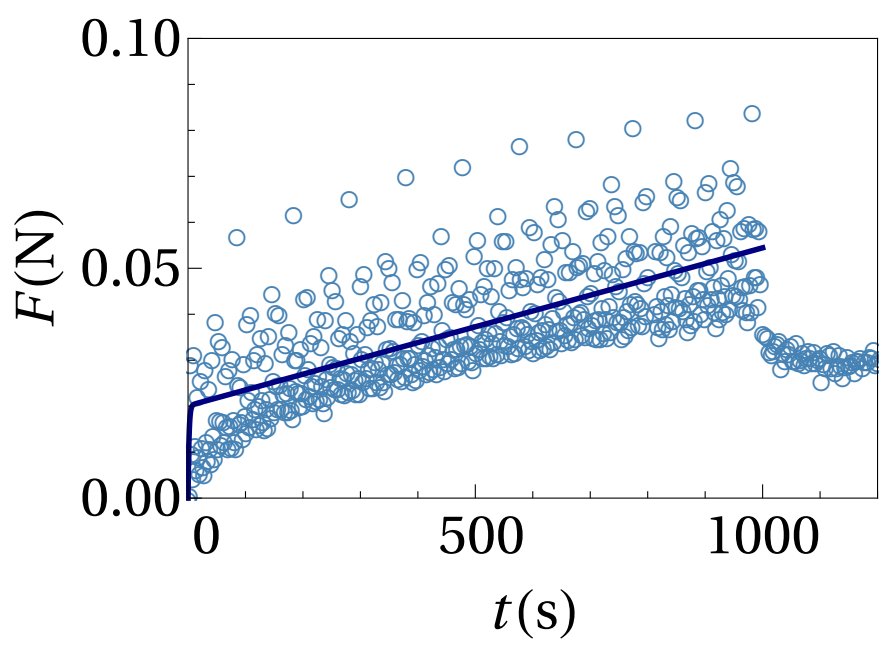

Figure 3.11: The measured normal force $F$ (blue circles) of a large-pore fibrin gel in response to slow ramp compression, see experiment 14 in Table 3.1 for the experimental conditions. The pressurizing time for the fluid pressure to build up to its maximal value is $t_{\perp}=20 \mathrm{~s}$. The blue curve is a fit of equation (3.29) to the measured normal force during compression, giving the permeability of the fibrin network $k$ and its longitudinal modulus $M$ as listed in Table 3.1.

nature of the polymerization process and the origin and purification process of fibrinogen.

The permeability estimates of the two $5 \%$ strain experiments are $k_{6}=$ $0.50(0.03) \mu \mathrm{m}^{2}$ and $k_{7}=0.23(0.01) \mu \mathrm{m}^{2}$, with the uncertainty of the best estimate in brackets. The first of these two estimates is more than one standard deviation from the average of the standard condition experiments. Therefore, the $5 \%$ strain permeabilities measurements suggest that possibly the permeability for $5 \%$ strain is significantly different from a gel which is $10 \%$ compressed. The best estimates of the Poisson's ratio of the small strain experiments, however, are $\nu_{6}=0.43(0.01)$ and $\nu_{7}=0.40(0.02)$, which both lie within one standard deviation of the standard condition experiments. Therefore, the 5\% Poisson's ratios suggest constancy of the Poisson's ratio in the range of $5-10 \%$ strain. Due to the small number of experiments, no conclusions can be drawn with respect to the influence of the magnitude of compression on $k$ and $\nu$. 
Concentration The standard condition (SC) in the compression experiments takes a fibrinogen solution with mass concentration $c=2 \mathrm{mg} / \mathrm{mL}$, see experiment $1 \mathrm{t} / \mathrm{m}$ 4. Two experiments were conducted with $c=4 \mathrm{mg} / \mathrm{mL}$, experiment 8 and 9 , to observe the influence of the concentration of fibrinogen. We expect a higher fibrinogen concentration $c$ to give rise to a smaller mesh size $\zeta$ and thus a lower permeability $k$. Assuming the mass density per unit length of fibrin fiber to be independent of the fibrinogen concentration $c$, the cubic lattice model gives $k \propto c^{-1}$, see section 3.6.2.

With the scaling relation between the permeability and the mass concentration of the fibrin network, we turn to the fit results. Taking the average of the best estimates of the fitted permeability of the standard condition experiments, we find $\bar{k}_{\mathrm{SC}}=0.27(0.17) \mu \mathrm{m}^{2}$, where in brackets is the standard deviation of the estimates, which we assume to measure the sample-to-sample variability of the permeability of different fibrin gels. The best estimates of the fitted permeability of the two 4 $\mathrm{mg} / \mathrm{mL}$ gels are $k_{8}=0.217(0.008) \mu \mathrm{m}^{2}$ and $k_{9}=0.063(0.002) \mu \mathrm{m}^{2}$, with the uncertainty in brackets. Given the sample-to-sample variation of the permeability, one of the fitted permeabilities of the $4 \mathrm{mg} / \mathrm{mL}$ experiments does not lie within one standard deviation, suggesting that possibly the permeabilities of $4 \mathrm{mg} / \mathrm{mL}$ experiments differs significantly from the standard condition experiments. Given the scaling relation derived above and the permeability estimation from the standard condition experiments, we would expect the permeabilities of the $4 \mathrm{mg} / \mathrm{mL}$ gels to equal $\bar{k}_{2} / 2=0.13(0.08) \mu \mathrm{m}^{2}$, with the sample-to-sample variation in brackets. The average of the two $4 \mathrm{mg} / \mathrm{mL}$ experiments is $\bar{k}_{4}=0.14 \mu \mathrm{m}^{2}$, therefore pointing to the validity of the scaling relation.

Next, the fitted Poisson's ratio from the standard condition experiments is $\bar{\nu}_{\mathrm{SC}}=$ $0.38(0.10)$. The best estimates for the $4 \mathrm{mg} / \mathrm{mL}$ gels are $\nu_{8}=0.18(0.05)$ and $\nu_{9}=$ $0.16(0.06)$, suggesting a significant difference in the Poisson's ratio. Due to the small number of experiments, however, no conclusions can be drawn on the influence of concentration on the permeability $k$ and the Poisson's ratio $\nu$.

Geometry To observe the influence of geometry, experiment 5 has been conducted, see Table 3.1, where the aspect ratio of the gel was $S=a / h=10$ instead of $S=20$ in the standard condition experiments. The fitted permeability of experiment 5 is $k_{5}=0.154(0.002) \mu \mathrm{m}^{2}$, whereas the standard condition experiments give an av- 


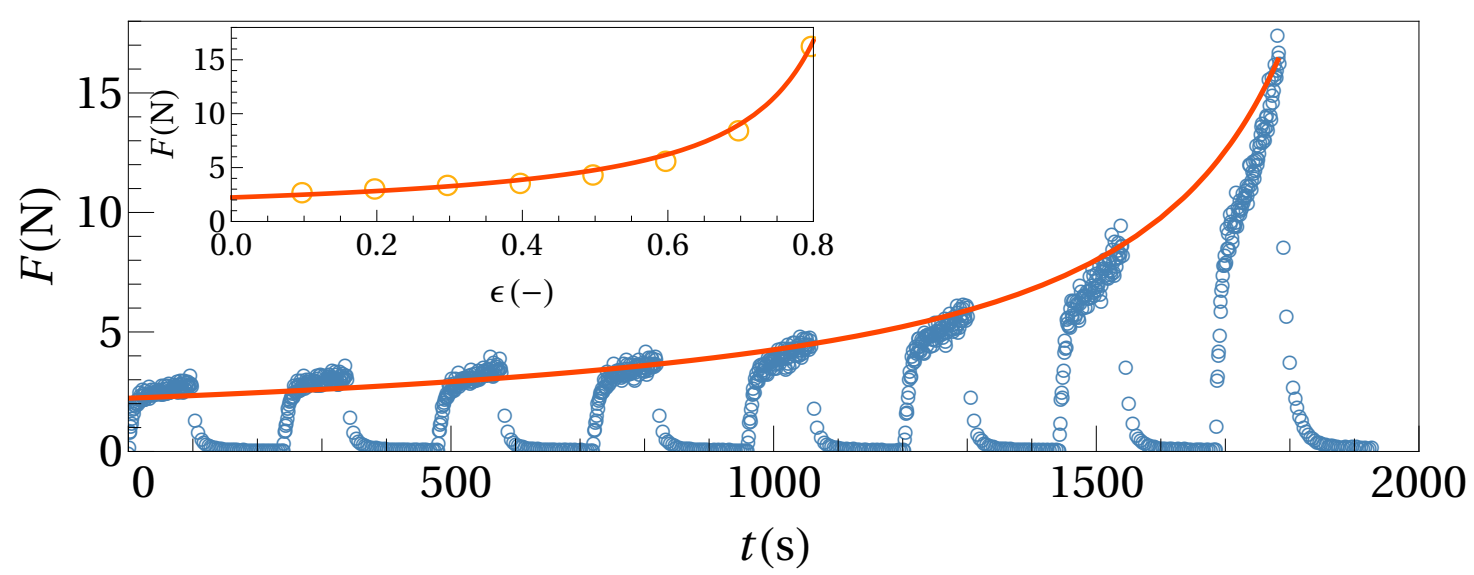

Figure 3.12: The measured normal force $F$ (blue circles) of a large-pore fibrin gel in response to eight consecutive ramp compression steps of $10 \%$ compressive strain each, see experiment 2 in Table 3.2 for the experimental conditions. The inset displays the maximal normal force during each compression step at the corresponding compressive strain (orange circles), which has been obtained by averaging the last $5 \%$ of data points of the compression step. The orange curve is a fit of equation (10) of the manuscript to the maximal normal force, giving the permeability of the fibrin network $k_{0}$ in the stress-free initial state and the proportionality constant of the Toll model $b$, as listed in Table 3.2.

erage permeability of $\bar{k}_{\mathrm{SC}}=0.27(0.17) \mu \mathrm{m}^{2}$, with the standard deviation of the best estimates of the permeability of the standard condition experiments in brackets, which is presumed to measure the sample-to-sample variation in permeability. Therefore, the permeability of experiment 5 does not seem to be significantly different from the permeability with $S=20$. The fitted Poisson's ratio of experiment 5 is $\nu_{10}=2.2(0.3)$, because the longitudinal modulus $M$ is fitted to be $160 \mathrm{~Pa}$ while the shear modulus $G$ is $227 \mathrm{~Pa}$, suggesting spontaneous contraction of the fibrin network. The reason for this awkward result is probably that the normal force increase during the pressurized phase is approximately $0.005 \mathrm{~N}$, while the uncertainty in the rheometer measurements is about $0.01 \mathrm{~N}$. Experiment 12 and 13 have also been conducted with an aspect ratio of $S=10$, however, also having increases of the normal force during the pressurized phase of less than $0.01 \mathrm{~N}$, but they do give physically reasonable fit values for the Poisson's ratio, $\nu=-0.56(0.42)$ and $\nu=-0.07(0.13)$, albeit with large uncertainties. Therefore, the anomalous value of the Poisson's ratio drawn from experiment 5 does not allow for a simple explanation. 
Strain rate The standard strain rate is taken to be $\dot{\epsilon} \equiv v / h=10^{-3} / \mathrm{s}$, with $v=1 \mu \mathrm{m} / \mathrm{s}$ the velocity of the upper plate and $h=1 \mathrm{~mm}$ the initial height of the sample. To observe the influence of strain rate, we conducted two experiments at $\dot{\epsilon}=5 \cdot 10^{-3} / \mathrm{s}$, see Figure 3.10, and two at $\dot{\epsilon}=0.1 \cdot 10^{-3} / \mathrm{s}$, see Figure 3.11. The standard condition (SC) experiments, experiment $1 \mathrm{t} / \mathrm{m} \mathrm{4}$, yield an average permeability $\bar{k}_{\mathrm{SC}}=0.27(0.17) \mu \mathrm{m}^{2}$, where the standard deviation due to sample-tosample variability is between brackets. The high strain rate experiments, experiment 10 and 11 , yield $k_{10}=0.120(0.007) \mu \mathrm{m}^{2}$ and $k_{11}=0.11(0.02) \mu \mathrm{m}^{2}$, which both fall within one standard deviation of the standard condition experiments. The low strain rate experiments, experiment 14 and 15 , yield $k_{14}=0.22(0.01) \mu \mathrm{m}^{2}$ and $k_{15}=0.183(0.007) \mu \mathrm{m}^{2}$, both within the standard deviation of the standard condition experiments. Strain rate therefore does not seem to significantly influence the permeability of the fibrin network, as one would expect, because the permeability is expected to depend primarily on the microscopic structure of the fibrin network, see section 3.6.2.

The Poisson's ratio of the SC experiments yields $\bar{\nu}_{1}=0.38(0.10)$, and the high speed experiments, experiment 10 and 11 , provide $\nu_{10}=0.46(0.03)$ and $\nu_{11}=1(14)$. Experiment 11 clearly gives an unreliable value for the Poisson's ratio. In this experiment the normal force decreases significantly during the pressurized phase, causing the fitted value of the longitudinal modulus to be fitted to zero, $M=0(4) \mathrm{kPa}$, though the uncertainty indicates that $M$ is in the order of $\mathrm{kPa}$, similar to experiment 10. The Poisson's ratio of experiment 10 falls within the variation of the SC experiments. The slow compression experiments, however, yield $\nu_{14}=-0.18(0.07)$ and $\nu_{15}=-0.54(0.13)$, clearly falling out of the sample-to-sample variation implied by the SCEs, see section 3.1 of the manuscript for interpretation of the strain rate dependence of the Poisson's ratio.

\section{Large compression of large-pore fibrin gels}

Additional to the small strain experiments on large-pore fibrin gels, see section 3.6.3, large strain compression experiments on large-pore fibrin gels have been conducted to observe the fibrin fiber network response to large compressive strains. The standard experimental conditions were equal to that of the small strain experiments: the fibrinogen concentration in the gels was taken to be $c=2 \mathrm{mg} / \mathrm{mL}$, the applied strain 
Table 3.2: Experimental conditions and fit results for the large compression experiments $(\epsilon \leq 80 \%)$ on large-pore fibrin. From the left to the right the columns provide: the tag given to each experiment, the concentration of fibrinogen $c$, the strain rate at which the gel is compressed $\dot{\epsilon}$, the fitted permeability $k$, and the fitted proportionality constant of the Toll model [179] $b$. All gels have height $h=1 \mathrm{~mm}$ and radius $a=20 \mathrm{~mm}$ before compression, and the estimation uncertainty is in brackets.

\begin{tabular}{c|cccc}
$\#$ & $c(\mathrm{~g} / \mathrm{L})$ & $\dot{\epsilon}\left(10^{-3} / \mathrm{s}\right)$ & $k\left(10^{-1} \mu \mathrm{m}^{2}\right)$ & $b(-)$ \\
\hline \hline 1 & 4 & 1.0 & $0.180(0.030)$ & $2.6(6.4)$ \\
2 & 4 & 1.0 & $0.195(0.008)$ & $12.0(1.4)$ \\
3 & 2 & 1.0 & $0.682(0.048)$ & $3.9(5.7)$ \\
4 & 2 & 1.0 & $1.070(0.050)$ & $22.0(2.4)$ \\
5 & 2 & 5.0 & $1.510(0.068)$ & $25.0(8.6)$ \\
6 & 2 & 5.0 & $1.400(0.029)$ & $76.0(4.2)$ \\
7 & 2 & 0.1 & $1.020(0.031)$ & $3.4(0.2)$ \\
8 & 2 & 0.1 & $0.943(0.045)$ & $3.0(0.3)$ \\
\hline \hline
\end{tabular}

rate was $\dot{\epsilon}=1 \cdot 10^{-3} / \mathrm{s}$, the initial gap size was $h=1 \mathrm{~mm}$ and the initial radius of the gel was $a=20 \mathrm{~mm}$. All gels were compressed up to $80 \%$ engineering strain in a stepwise fashion, see Figure 3.12. Each step comprised 10\% strain and after each step the gel was allowed to relax, i.e., the measured normal force decreased after compression to a constant value. Figure 3.12 shows that the different compression steps have a similar normal force response, although the maximal normal force increases with compression. Therefore, we assume that also under large compression the gel enters the pressurized phase during compression.

In section 3.6.1, we assumed the gel network to be linear elastic, and to have a constant permeability, both rather poor assumptions for a fibrin gel under large compression. Therefore, as explained in section 2 of the manuscript, we assume a phenomenological form of the normal force as a function of the compressive strain in the pressurized phase, see equation (10) of the manuscript.

We assume the Young's modulus of a single fibrin fiber to be in the order of MPa: $E_{\mathrm{f}}=1 \mathrm{MPa}$ [184]. The volume fraction of fibrin fiber before compression is $\phi_{0}=f c / \rho_{\text {fibrinogen }}$, with $\rho_{\text {fibrinogen }}=1.4 \cdot 10^{3} \mathrm{~kg} / \mathrm{m}^{3}$ the mass density of pure fibrinogen [173], $f=5.0$ the volume which a fibrin fiber encompasses relative to the volume of fibrinogen molecules in the fiber [185], and $c$ the overall concentration of 
Table 3.3: Experimental conditions and fit results for compression experiments on smallpore fibrin gels. From left to right, the columns provide: the tag given to each experiment, the concentration of fibrinogen $c$, the initial height $h$ and radius $a$ of the gel before compression, the strain rate at which the gel is compressed $\dot{\epsilon}$, the amount of engineering strain put on the gel after compression $\epsilon_{\mathrm{e}}$, the fitted permeability $k$, the fitted longitudinal modulus $M$, the measured shear modulus just before compression $G_{0}$ from small-strain rheometry, the augmented shear modulus during compression $G_{\mathrm{C}}$, and the exponential relaxation time $t_{\perp} / 12$ with $t_{\perp}$ the pressurizing time. The strain rate is $\dot{\epsilon}=10^{-3} / \mathrm{s}$ in all experiments, and the uncertainty is given in brackets.

\begin{tabular}{c|cccccccccc}
\hline$\#$ & $c(\mathrm{~g} / \mathrm{L})$ & $h(\mathrm{~mm})$ & $a(\mathrm{~mm})$ & $\epsilon_{\mathrm{e}}(\%)$ & $k\left(10^{-3} \mu \mathrm{m}^{2}\right)$ & $M(\mathrm{kPa})$ & $G_{0}(\mathrm{kPa})$ & $G_{\mathrm{c}}(\mathrm{kPa})$ & $t_{\mathrm{c}}(\mathrm{s})$ & $t_{\perp} / 12(\mathrm{~s})$ \\
\hline \hline 18 & 2 & 0.5 & 20 & 10 & $6.64(0.24)$ & $6.1(2.7)$ & 0.04 & $0.19(0.02)$ & $6.4(0.6)$ & 11 \\
19 & 2 & 1.0 & 20 & 10 & $3.86(0.15)$ & $0(4.6)$ & 0.05 & $0.59(0.02)$ & $7.9(0.3)$ & 25 \\
20 & 2 & 1.0 & 20 & 10 & $4.75(0.20)$ & $0(4.1)$ & 0.04 & $0.50(0.02)$ & $7.9(0.3)$ & 24 \\
21 & 2 & 1.0 & 20 & 5 & $3.37(0.11)$ & - & 0.05 & $0.49(0.01)$ & $6.2(0.3)$ & 35 \\
22 & 2 & 1.0 & 20 & 5 & $3.40(0.13)$ & - & 0.04 & $0.50(0.02)$ & $6.7(0.3)$ & 34 \\
23 & 4 & 1.0 & 20 & 5 & $0.73(0.05)$ & - & 0.24 & $1.03(0.02)$ & $5.8(0.3)$ & 77 \\
24 & 4 & 1.0 & 20 & 5 & $0.73(0.06)$ & - & 0.19 & $0.90(0.02)$ & $9.3(0.2)$ & 88 \\
25 & 6 & 1.0 & 20 & 5 & $0.22(0.04)$ & - & 0.47 & $1.22(0.03)$ & $5.6(0.3)$ & 211 \\
26 & 6 & 1.0 & 20 & 5 & $0.30(0.04)$ & - & 0.49 & $1.39(0.04)$ & $5.9(0.3)$ & 137 \\
27 & 6 & 1.0 & 20 & 5 & $0.48(0.05)$ & - & 0.36 & $1.08(0.03)$ & $5.7(0.3)$ & 110 \\
28 & 6 & 1.0 & 10 & 5 & $0.80(0.07)$ & - & 0.36 & $1.17(0.05)$ & $10.8(0.4)$ & 62 \\
29 & 6 & 1.0 & 10 & 5 & $0.43(0.04)$ & - & 0.40 & $1.99(0.07)$ & $9.9(0.4)$ & 68 \\
30 & 6 & 1.0 & 10 & 5 & $0.74(0.06)$ & - & 0.49 & $1.36(0.05)$ & $9.9(0.4)$ & 57 \\
31 & 8 & 1.0 & 20 & 5 & $0.22(0.03)$ & - & 0.65 & $1.69(0.04)$ & $7.2(0.3)$ & 152 \\
32 & 8 & 1.0 & 20 & 5 & $0.16(0.04)$ & - & 0.70 & $1.24(0.03)$ & $9.8(0.4)$ & 295 \\
33 & 10 & 1.0 & 20 & 10 & $0.17(0.05)$ & - & 0.97 & $1.32(0.05)$ & $14.4(1.0)$ & 264 \\
\hline \hline
\end{tabular}

fibrinogen in the gel. As the fibrin fiber network sticks to the plates, one can approximate the average volume fraction as $\phi=\phi_{0} /(1-\epsilon)$, where we ignore the bulging of the gel since $S=a / h \gg 1$.

To fit equation (10) of the manuscript to the measured normal force in the pressurized phase, in principle one needs to know when the pressurized phase sets in. This is unknown outside of the linear regime.

As the exponential relaxation time in the first compression step is in the order of seconds, however, see Table 3.1, we assume that at the end of each compression step, with $t_{\mathrm{e}}=20100 \mathrm{~s}$, the gel has entered the pressurized phase, and we fit equation (10) of the manuscript to the maximal normal forces. Due to the uncertainty in the measured normal force, we take the last five percent of data points of a given compression step and average both the normal force and the strain of the different data points, to 
obtain a single data point per compression step, see the inset of Figure 3.12.

Two of the large compression experiments were performed at standard conditions, two at high strain rate $\dot{\epsilon}=5 \cdot 10^{-3} / \mathrm{s}$, two at low strain rate $\dot{\epsilon}=0.1 \cdot 10^{-3} / \mathrm{s}$, and two with a doubled concentration of fibrinogen $c=4 \mathrm{mg} / \mathrm{mL}$, see Table 3.2. Comparing experiment 1 and 2 to experiment 3 and 4 , the fitted prefactors $b$ do not appear to be influenced by the fibrinogen concentration $c$. This result confirms the assumed form of the stress response of the fibrin fiber network as that of a randomly structured fiber network in the Toll model [179], where the concentration of fibrinogen is taken into account by the volume fraction of fibrin fibers $\phi$. Comparison of experiment $3-8$, where the influence of strain rate is probed, suggests a monotonous increase of $b$ with the strain rate, see Figure 4 of the manuscript. This increase is analogous with the findings described in section 3.6.3, which showed that for higher strain rate the gel responds more like a volume-conserving solid. The increase in strain rate suggests that for the strain rate going to zero the proportionality constant $b$ of the fiber network response could be of order 1 or less. The dependence of the normal force to strain rate in large compression experiments shows that the strain rate dependence of the mechanical response of the fibrin fiber network holds both for small and large compressive strain.

Next, as noted in section 3.6.3, we expect the permeability to scale as $k \propto c^{-1}$, and we do not expect it to depend on the strain rate, which determines the fluid velocity through the fiber network, as the permeability is supposed to be determined by the architecture of the network. The network architecture would depend on the amount of compressive strain, but not on the strain rate. Therefore, we expect the fitted permeability at zero compression $k_{0}$ to be independent of the strain rate, although we do expect sample-to-sample variation, see section 3.6.3. Experiment 3-8 provide permeabilities which indeed fall within approximately one standard deviation of the permeability of experiment 1-4 of the small strain experiments under standard conditions, see Table 3.1 and 3.2. All fitted permeabilities are at the lower end, however, which seems somewhat unexpected. Moreover, given the scaling relation $k \propto c^{-1}$, the permeability of experiment 1 and 2 with $c=4 \mathrm{mg} / \mathrm{mL}$ would be expected to be approximately half of that of experiment $3-8$. This is not confirmed by the fitted values, however. Due to the low number of experiments no conclusions can be drawn. 


\section{Compression of small-pore fibrin gels}

Next to the compression experiments on large-pore fibrin gels, see section 3.6.3, gels of small-pore fibrin networks have been compressed while measuring the normal force. The standard conditions were, similar to those in section 3.6.3 and 3.6.3, a fibrinogen concentration of $c=2 \mathrm{mg} / \mathrm{mL}$, a strain rate of $\dot{\epsilon}=10^{-3} / \mathrm{s}$, an initial gel height of $h=1 \mathrm{~mm}$, and an initial radius of $20 \mathrm{~mm}$. We increased the concentration of fibrinogen up to $10 \mathrm{mg} / \mathrm{mL}$ to observe its influence on the permeability. Moreover, we varied the geometry of the gels to test the validity of the approximate solution presented in section 3.6.1. Below, we first discuss the numerical details of the fitting procedure. Afterwards, we consider the influence of fibrinogen concentration and geometry on the permeability and shear modulus of small-pore fibrin networks.

Strain stiffening As noted in section 2 of the manuscript, we accommodate for strain stiffening by replacing $G t \rightarrow \int_{0}^{t} G\left(t^{\prime}\right) \mathrm{d} t^{\prime}$ where $G$ enters in equation (3.29), with $G(t)$ as given in equation (7) of the manuscript. For the numerical fit routine, we use a sigmoidal function with very high power $n=33$ to interpolate approximately stepwise and analytically from $G_{0}$ to the augmented shear modulus $G_{\mathrm{c}}$. This gives for the time-dependent shear modulus

$$
G(t)=G_{0}+\left(G_{\mathrm{c}}-G_{0}\right) \frac{\left(t / t_{\mathrm{c}}\right)^{n}}{1+\left(t / t_{\mathrm{c}}\right)^{n}} .
$$

All small-pore fibrin experiments can be described well by fitting the permeability $k$, the augmented shear modulus $G_{\mathrm{c}}$, the onset time for strain stiffening $t_{\mathrm{c}}$, and, if possible, the longitudinal modulus $M$, see Table 3.3.

In experiment 18, the fit routine was able to converge with a meaningful value of the longitudinal modulus $M=6.2(2.7) \mathrm{kPa}$. The reason for convergence is that in this experiment the initial height of the gel was $h=0.5 \mathrm{~mm}$, causing a relatively short exponential relaxation time $t_{\perp} / 12=h^{2} \eta / 12 k G_{\mathrm{c}}$ of $11 \mathrm{~s}$. Therefore, during a significant portion of compression the gel is to good approximation in the pressurized phase, in which the increase in normal force is solely due to the longitudinal modulus, see equation (3.29). For experiment 21-33, we put $M=0$ by hand, because otherwise the fit routine does not converge. This presumption is also justified, however, for the following reasons. Arguably, the non-convergence is due to the negligible effect 


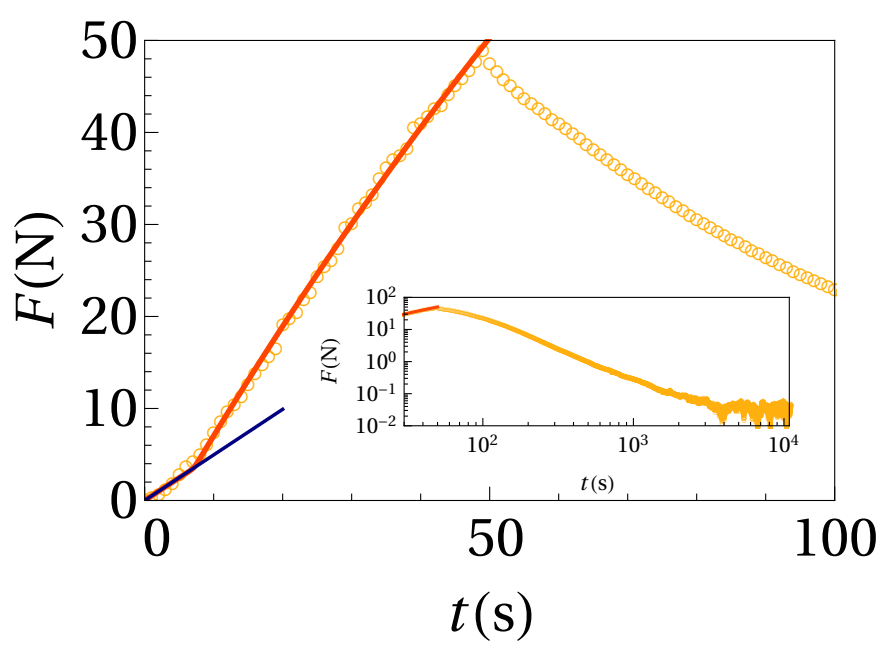

Figure 3.13: The measured normal force $F$ (orange circles) of a small-pore fibrin gel with high fibrinogen concentration, see experiment 31 in Table 3.3 for the experimental conditions. The pressurizing time for the fluid pressure to build up to its maximal value is $t_{\perp}=1824 \mathrm{~s}$. The orange curve is a fit of equation (3.29) to the measured normal force during compression, giving the permeability of the fibrin network $k$, the onset time of strain stiffening $t_{\mathrm{c}}$ and the augmented shear modulus $G_{\mathrm{c}}$ as listed in Table 3.3. Before the onset time of strain stiffening, the normal force follows the time dependence expected for a volume-conserving solid with the measured initial shear modulus $G_{0}$. The inset shows an extended process of gel relaxation after compression stops.

of the mechanical response, $\pi a^{2} M \epsilon_{\mathrm{e}}$ with $a$ the initial radius of the gel and $\epsilon_{\mathrm{e}}$ the amount of strain after compression, in the pressurized phase. Moreover, these experiments all have $h=1 \mathrm{~mm}$, implying a longer pressurizing time than in experiment 18. In part of the experiments, the gel is compressed with only $5 \%$ engineering strain $\epsilon=0.05$, reducing the mechanical response contribution even further. Finally, with increasing concentration the relaxation time $t_{\perp}$ seems to increase, thereby increasing the time needed to enter the pressurized phase and decreasing the influence of $M$, see Figure 3.13 for a high fibrinogen concentration experiment with $M=0$.

Concentration and geometry To observe the influence of the concentration of fibrinogen $c$ we conducted experiments with $c=2,4,6,8,10 \mathrm{mg} / \mathrm{mL}$, see Table 3.3 for the results and Figure 3.13 for a high fibrinogen gel. The permeability $k$ decreases with increasing fibrinogen concentration $c$, see Figure (7) of the manuscript.

Up to the increase of the shear modulus at $t_{\mathrm{c}}$, the fibrin gels deform in an approxi- 
mately volume-conserving manner. Therefore, the compression up to $t=t_{\mathrm{c}}$ can be considered as a shear deformation of the sample. The tangential radial stress at the sample-plate interface therefore shears the sample. Within the gel the shear stress is lower because the gel can bulge out. The tangential stress at the sample-plate interface before stiffening is given as $\sigma_{\mathrm{n}, r z}=G_{0}\left(\partial_{z} U+\partial_{r} W\right)=G_{0} \partial_{z} U$, since $W$ is independent of $r$, as we presumed in this entire analysis. The radial deformation $U$ grows linearly with the radial coordinate $r$, see equation (3.11). Therefore, we average the tangential stress over the gel plate interface to obtain the critical stress: $\left.\sigma_{\mathrm{c}} \propto\left(1 / \pi a^{2}\right) \int_{0}^{a} \mathrm{~d} r 2 \pi r \sigma_{r z}^{\prime}\right|_{z=h, t=t_{\mathrm{c}}}$, giving equation (9) of the manuscript, and the dependence of $\sigma_{\mathrm{c}}$ on the concentration of fibrinogen $c$ is given in Figure 6 of the manuscript.

Experiments 25-30 have a fibrinogen concentration of $c=6 \mathrm{mg} / \mathrm{mL}$, whereas experiments 25-27 have an initial radius of $a=20 \mathrm{~mm}$ and experiments 28-30 have $a=10 \mathrm{~mm}$. According to equation (3.29), a difference in the magnitude of the normal force $F_{\mathrm{N}} \propto a^{4}$ is to be expected, but no difference in the fitted permeability $k$, the shear modulus $G_{\mathrm{c}}$ and the critcial time $t_{\mathrm{c}}$. The three fit parameters are all of the same order, their differences are probably due to sample-to-sample variation, and the coefficient of determination exceeds 0.9992 for every experiment. Therefore, our model seems to account correctly for the influence of geometry.

Shear modulus In section 3.6.3 we found the measured normal force in all largepore fibrin compression experiments during compression to be explained by the approximate solution of section 3.6.1, while using the measured value of the shear modulus $G_{0}$ just before compression. For small-pore fibrin gels, however, we found the shear modulus to increase at a critical stress and to remain constant afterwards. In this section, we also consider the evolution of the shear modulus after compression.

For large-pore fibrin gels undergoing large compression, see section 3.6.3, the shear modulus has been measured before and after compression, see for example Figure 3.14. Before compression, the shear modulus has a constant value $G_{0}$. After compression, it first decreases rapidly. Subsequently, it increases very slowly and can be regarded as approximately constant, i.e., as if the gel is relaxed. Just after compression, the shear modulus seems to be at approximately the same value as before compression, suggesting the shear modulus in large-pore fibrin gels to be approximately 


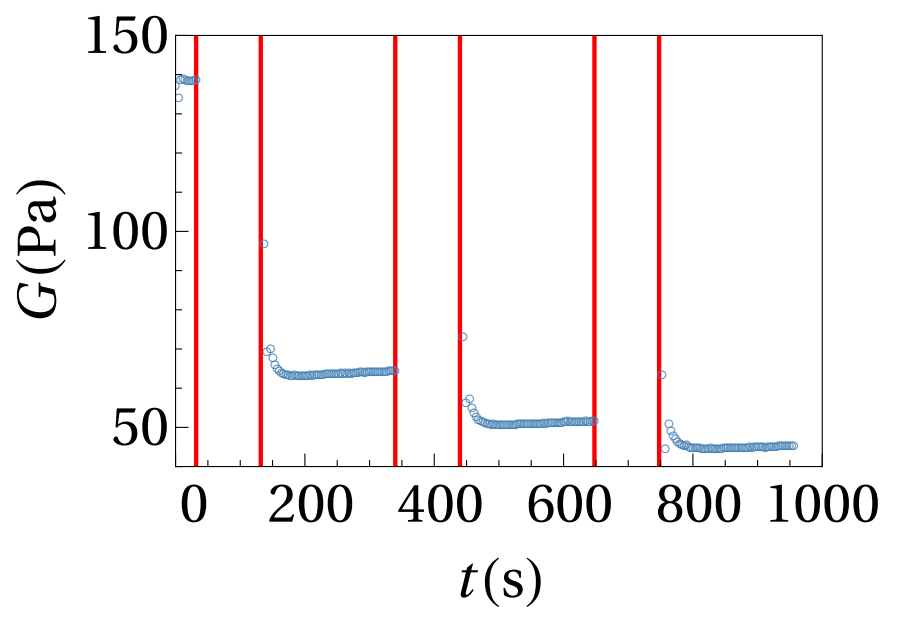

Figure 3.14: The measured shear modulus $G$ (blue dots) before and after three compression steps of $10 \%$ compressive strain for a large-pore fibrin sample. The beginning and end of a compression step are denoted by a red line. Before each compression step the shear modulus is (approximately) constant. After each step the shear modulus decreases rapidly to a smaller value than before compression.

constant during compression, as we assumed in section 3.6.3. With each compression step, the magnitude of the relaxed shear modulus is decreased compared to its value before compression, in accordance with literature [167].

Combining the results from section 3.6.3, 3.6.3 and the shear modulus measurements just after compression, we can form a coherent picture of the evolution of the shear modulus during and after the compression of fibrin gels, see Figure 3.15. Before compression, the gel has a constant shear modulus which reflects that the gel is in equilibrium. As soon as compression starts it is first compressed in a volume-conserving manner because the (low) permeability of the gel prevents fluid to be squized out instantaneously. In this phase the shear modulus may increase around an onset stress $\sigma_{\mathrm{c}}$ in the network, see equation (9) of the manuscript. If fluid starts to flow out before the gel is stressed to $\sigma_{\mathrm{c}}$, the shear modulus remains constant throughout the whole of the ramp compression, as with the large-pore compression experiments in section 3.6.3. If $\sigma_{\mathrm{c}}$ is reached while being in the volume-conserving phase, the shear modulus can increase significantly and remains so during the rest of compression, see section 3.6.3. After compression, the shear modulus relaxes to its static value.

The evolution of the shear modulus of a fibrin gel of Figure 3.15, and the difference in stiffening depending on whether the gel reaches the onset stress $\sigma_{\mathrm{c}}$ in the 


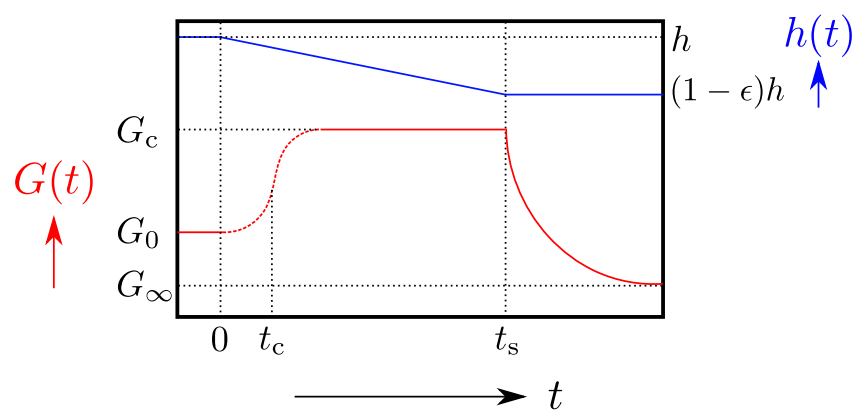

Figure 3.15: Schematic of the development of the shear modulus $G(t)$ in response to changes in the height $h(t)$ of a ramp-compressed fibrin gel during and after compression. Initially, for $t<0$ the shear modulus has magnitude $G_{0}$. As compression commences, the shear modulus increases to $G_{\mathrm{c}}$, with the point of maximum increase at the onset time $t_{\mathrm{c}}$. The difference between $G_{\mathrm{c}}$ and $G_{0}$ is negligibly small for large-pore fibrin gels but may be significant for small-pore fibrin gels. This difference is probably due to the much shorter volume-conserving phase of large-pore fibrin gels. After compression stops at $t_{\mathrm{e}}$, the shear modulus decreases to its static value $G_{\infty}$, which is determined by the compressive strain.

volume-conserving phase or not, suggests that the occurrence of strain stiffening has a strong connection with the flow of fluid through the fibrin network. In the volumeconserving phase, when no fluid flows relative to the fibrin network, the shear modulus shows compressive stiffening, as most materials do when compressed. As the gel transitions into the pressurized phase and the fluid velocity relative to the network increases to a nonzero value, however, the modulus becomes fixed. Therefore, it seems as if flow of fluid through the network prevents further stiffening of the shear modulus. After compression stops, however, and the relative velocity starts to decrease to zero, the shear modulus also decreases to its new equilibrium value. 


\section{Chapter 4}

\section{Plate-sphere compression}

The results of this chapter are contained in:

M.T.J.J.M. Punter \& B.M. Mulder. "Uniaxial compression tests using a spherically curved contact surface: a perturbative approach". In: preparation. 


\section{Abstract}

Conventional uniaxial compression tests employ testing machines where flat surfaces touch the flat ends of a cylindrical specimen. Within this geometry, misalignment of the surfaces may induce significant error in the determination of, e.g., the Young's modulus. As a novel geometry, we investigate the compression of a cylinder with a flat surface from below and a spherical surface from above. Using a perturbative analysis of the elastostatic equations in displacement form, we report for this geometry a polynomial full-field solution for the displacement and stresses of a frictionless linear elastic material. This solution provides the exact expression for the required load for any shape of the cylinder. Our perturbative approach is fit for wider application in the field of elasticity theory. 


\subsection{Introduction}

For the determination of the mechanical properties of a material, e.g., the Young's modulus, a commonly used method is to press a sample between two parallel flat surfaces in a uniaxial compression test [186-189]. A practical problem in compression testing is the friction which exists between the sample and the plates. It causes the relation between the engineering strain and the load to be nontrivial, causing discrepancy between the measured apparent Young's modulus and the intrinsic Young's modulus [177, 190-192]. For materials which can be shaped at will, e.g., metals, the solution is to use a long cylinder because the end effects decay by Saint Venant's principle, causing the middle to be in a state of homogeneous strain [193]. For materials with shape limitations, on the other hand, lubrication of the ends can be a solution $[188,189]$.

Recent experimental and numerical studies suggest, however, that apart from friction, in the case of an elastic-plastic material, an important source of discrepancy between the apparent and the intrinsic Young's modulus is misalignment of the two flat plates straining the sample [194]. The misalignment causes non-axisymmetric deformation of the compressed cylinder with local zones of high plastic deformation [195]. These local zones of high plastic deformation blur the cross-over between the regimes of elastic and plastic compression, thereby introducing error in the determination of the Young's modulus. Moreover, for shape-limited materials with medium to high aspect ratios (radius/height), misalignment has a strong influence because the end effects are significant.

Can misalignment problems be evaded by choosing a different geometry of the compression test? To explore alternative testing machine geometries, we consider the compression of a frictionless cylinder not with two flat surfaces, but with a flat surface from below and a spherical surface from above, see Figure 4.1A. The use of a spherical surface can be advantageous, because the spherical symmetry of the surface circumvents the need to align the two surfaces in the first place. Also, it could reduce the effect of tilt of the spherical surface due to testing machine wear. In the case of elastic-plastic materials, the spherical symmetry could make regions of plastic deformation less concentrated, thereby reducing error in the determination of the Young's modulus. For shape-limited materials, the end effect of sphericity of the upper plate in lubricated compression can be taken into account exactly, as we show here. Moreo- 
ever, the use of a spherical surface can facilitate the pinning of a lubricated cylindrical sample.

A. Uncompressed

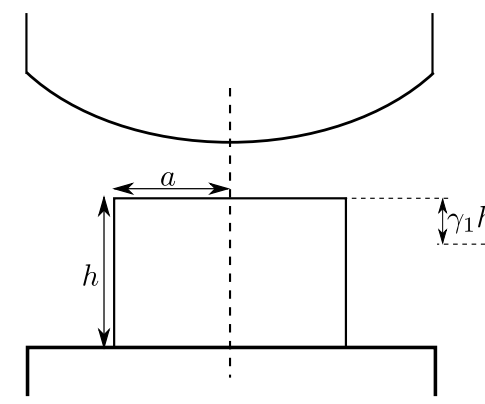

B. Plate-sphere compression

Figure 4.1: A) An initially unstressed and frictionless cylinder of radius $a$ and height $h$ of an isotropic and homogeneous linear elastic material in a compression testing machine with plate-sphere geometry. The dotted line gives the axis of symmetry of the cylinder. B) During compression, the tip of the surface is moved over the axis of symmetry and translates the center of the upper end of the cylinder over a distance $\gamma_{1} h$. The circular boundary of the upper end of the cylinder is translated over a distance $h\left(\gamma_{1}-\gamma_{2}\right)$, where $\gamma_{2} h=a^{2} / 2 R$ is determined by the radius of curvature $R$ of the spherical surface. For medium to high aspect ratio $S \equiv a / h$, the entire sample is in a state of inhomogeneous strain due to the curvature of the upper plate.

To good approximation, a spherical surface can be regarded as a paraboloid. Therefore, we study theoretically the compression of a frictionless cylinder made of a linear elastic material at any aspect ratio, using a flat surface from below and a paraboloidal shaped surface from above, where the tip of the paraboloid is exactly aligned with the axis of symmetry of the cylinder. Under these conditions, the sample will be strained inhomogeneously, see Figure 4.1B. To find the displacement field, we employ a perturbative expansion of the elastostatic equations in displacement form by regarding, essentially, the ratio of the shear modulus over the bulk modulus as a small parameter. We solve the equations of motion to all orders in the perturbation with the radial traction-free boundary condition in weak form. We find a polynomial solution which becomes, independent of Saint Venant's principle, exact in the limit of high aspect ratio. Using a corrective solution, we find the exact solution for any aspect ratio. The corrective solution does not add to the load, however, rendering the calculated load from the polynomial solution exact for all aspect ratios. Therefore, our solution can be used in a plate-sphere testing machine to determine the Young's modulus of materials of any cylindrical shape, while possibly avoiding problems associated to 
misalignment.

We first introduce our perturbative approach of the elastostatic equations. Next, we use this approach to find the displacement field of a cylinder under plate-sphere compression.

\subsection{Perturbation expansion around an incompressible solid}

In this section we employ perturbation theory to reformulate the equilibrium equations of motion and appurtenant boundary conditions (BCs), i.e., the elastostatic equations, for a body of a linear elastic isotropic and homogeneous material. In the next section, we use this approach to find the solution for frictionless plate-sphere compression.

For a body of a homogeneous and isotropic linear elastic material without body forces acting on it, the elastostatic equations can be written as [196]

$$
\begin{aligned}
0 & =\nabla(\nabla \cdot \mathbf{U})+\epsilon \nabla^{2} \mathbf{U}, \text { in } \mathcal{B}, \\
\mathbf{U}(\mathbf{x}) & =\mathbf{h}(\mathbf{x}), \text { on } \overline{\partial \mathcal{B}_{1}}, \\
n_{j} \sigma_{i j} & =l_{i}(\mathbf{x}), \text { on } \partial \mathcal{B}_{2},
\end{aligned}
$$

where $\mathbf{U}(\mathbf{x})$ is the displacement field, $\mathcal{B}$ is an open region of Euclidean $2 \mathrm{D}$ or $3 \mathrm{D}$ space occupied by the body with a bounding surface $\partial \mathcal{B}=\overline{\partial \mathcal{B}_{1}} \cup \partial \mathcal{B}_{2}$, and $\mathbf{h}(\mathbf{x})$ and $\mathbf{l}(\mathbf{x})$ are, respectively, prescribed displacements and tractions. We assumed the socalled chi-modulus to be nonzero $\chi \equiv K+G / 3 \neq 0$, and defined the deviation of the material from an incompressible material, the so-called compressibility parameter, as $\epsilon \equiv 1-2 \nu=G / \chi$, with $K$ the bulk modulus, $G$ the shear modulus and $\nu$ the Poisson ratio.

Considering the chi-modulus $\chi$ and the compressibility parameter $\epsilon$ as independent elastic constants, we treat $\epsilon>0$ as a small perturbative parameter, and expand the displacement field, the prescribed displacements and the tractions around the incompressible limit [197-200]. For example, we write for the displacement field

$$
\mathbf{U}(\mathbf{x})=\sum_{n=0}^{\infty} \epsilon^{n} \mathbf{U}^{(n)}(\mathbf{x}),
$$


where $\mathbf{U}^{(n)}$ is the $n$-th order displacement field. From the constitutive equations, we find the stress fields as

$$
\frac{\sigma_{i j}}{\chi}=e_{l l}^{(0)} \delta_{i j}+\sum_{n=1}^{\infty} \epsilon^{n}\left(e_{l l}^{(n)} \delta_{i j}+2 d_{i j}^{(n-1)}\right),
$$

where $e_{i j}^{(n)}=\frac{1}{2}\left(\nabla_{i} U_{j}^{(n)}+\nabla_{j} U_{i}^{(n)}\right)$ and $d_{i j}^{(n)} \equiv e_{i j}^{(n)}-\delta_{i j} e_{l l}^{(n)} / 2$ are, respectively, the strain tensor and the $2 \mathrm{D}$ deviatoric strain tensor of the $n$-th order displacement field, and repeated indices imply summation: $\epsilon_{l l}^{(n)}=\nabla \cdot \mathbf{U}^{(n)}$.

This expansion amounts to treating the body as the superposition of a nearly incompressible body, defined by the combination of the zeroth and first order, with an infinite number of compressible body's, defined by each higher order. All body's are coupled in a linear hierarchy. We note that this expansion is not unique, as one could expand in any $\eta \equiv \epsilon f(\epsilon)$, with $f(\epsilon) \rightarrow 1$ if $\epsilon \rightarrow 0$. Our choice, however, gives a convenient linear hierarchical form of the perturbative equations of motion.

As the prescribed displacements and tractions are also expanded, our approach allows for a systematic analysis of BCs of different order of magnitude in $\epsilon$. Generally, however, prescriptions will be independent of $\epsilon$, and are thus treated at the lowest order nearly incompressible body, implying all higher order compressible body's to obey homogeneous boundary conditions, similar to other recursive solution schemes [201].

From the force balance, equation (4.1), we obtain

$$
0=\nabla e_{l l}^{(0)}+\sum_{n=1}^{\infty} \epsilon^{n}\left(\nabla e_{l l}^{(n)}+\nabla^{2} \mathbf{U}^{(n-1)}\right)
$$

which shows that nonhomogeneous deformation in the $n$-th order body induces gradients in the local dilatation of the $(n+1)$-th order body. In principle, we expanded the elastostatic equations, equation (4.1)-(4.3) in an infinite number of static problems, which can be easier to solve. For illustration of the perturbative expansion, we treat the standard problems of isotropic, constrained and plate-plate compression in 4.5.1.

Truncating the expansions in equation (4.4) and (4.5) at first order, we find the constitutive equations of a nearly incompressible body

$$
\sigma_{i j}=-\delta_{i j} p+2 G e_{i j}^{\prime(0)}
$$


A. Boundary value problems

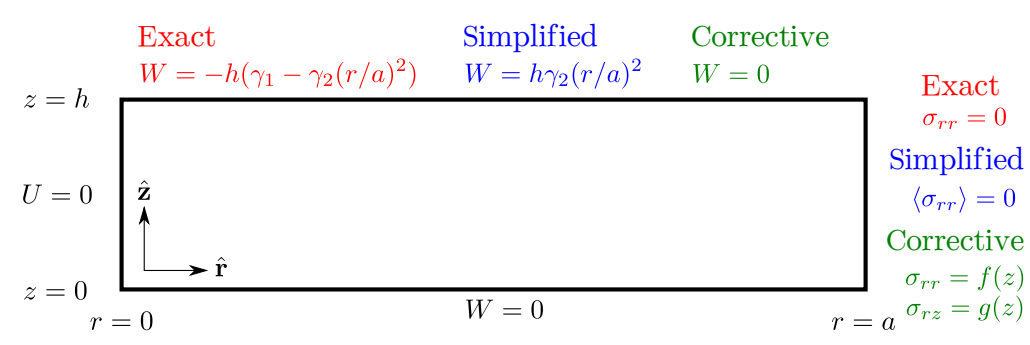

B. Solution flow chart

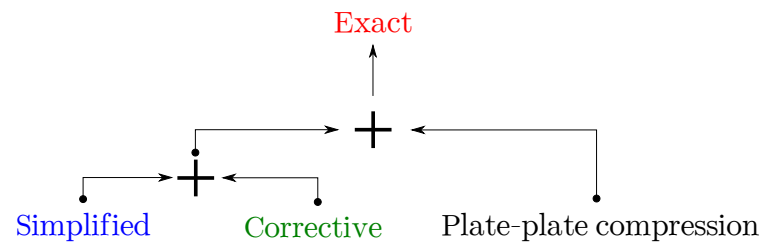

Figure 4.2: A) Overview of boundary value problems for the displacement field $\mathbf{U}(r, z)=$ $U(r, z) \hat{\mathbf{r}}+W(r, z) \hat{\mathbf{z}}$ of a cylinder of height $h$ and radius $a$. The exact boundary value problem (red) is that of a free frictionless cylinder compressed by a spherical surface with radius of curvature $R \equiv a^{2} / 2 h \gamma_{2}$, giving a resulting engineering strain $\gamma_{1}$ at the cylinder axis, see Figure 4.1. The simplified problem (blue) obeys the weak form of the radial traction-free boundary condition and has $\gamma_{1}=0$ for clarity. The corrective solution (green) generates a radial[tangential] traction distribution $f(z)[g(z)]$ on the free boundary symmetrical[antisymmetrical] in $z=0$. Unless specified otherwise, the tangential tractions vanish at each boundary $\sigma_{r z}=G\left(\partial_{z} U+\partial_{r} W\right)=0$. B) Flow chart of the relations between the different boundary value problems. Plate-plate compression is the solution of a standard uniaxial compression test with engineering strain $\gamma_{1}$, see 4.5.1.

provided one identifies $p=-\left(K e_{l l}^{(0)}+G e_{l l}^{(1)}\right)$ as the pressure in the body, with $e_{i j}^{\prime(n)} \equiv e_{i j}^{(n)}-\delta_{i j} e_{l l}^{(n)} / 3$ the $3 \mathrm{D}$ deviatoric strain. In this constitutive equation, the stress scale of the zeroth and first order contribution, $K=\chi(1-\epsilon / 3)$ and $G=\chi \epsilon$ respectively, are linearly independent, implying as force balance equations $0=\nabla e_{l l}^{(0)}$ and $0=\nabla e_{l l}^{(1)}+\nabla^{2} \mathbf{U}^{(0)}$. If $e_{l l}^{(0)}=0$, we obtain the equations of motion of an incompressible solid [196]. Usually, the pressure of an incompressible solid is introduced as a Lagrange multiplier [21], but it follows naturally from our perturbative expansion.

Using the perturbative system of equations, we solve the problem of plate-sphere compression in the next section. 


\subsection{Plate-sphere compression}

In this section, we consider a cylinder of length $h$ and radius $a$ compressed by a flat plate from below and a paraboloidal plate from above. At the ends the displacements are prescribed by the shape of the plates and the condition of frictionless contact. The displacement imposed by the upper plate, assuming full contact, is a combination of a translational engineering strain $\gamma_{1}$, similar to plate-plate compression, and a curvature-induced strain $\gamma_{2}$, see Figure 4.1. At the free boundary the tractions vanish, see Figure 4.2A for the appurtenant exact boundary value problem (BVP), where we assume axial symmetry, $U(r, z)$ is the radial translation and $W(r, z)$ is the vertical translation of the displacement field $\mathbf{U}(r, z)=U(r, z) \hat{\mathbf{r}}+W(r, z) \hat{\mathbf{z}}$.

In principle, the exact solution to this problem is known [19, 202, 203], but is in the form of infinite series whose coefficients must be determined by matrix inversion. Seeking an exact closed-form expression for the engineering stress, we treat the equilibrium equations of motion perturbatively, as outlined in the previous section. To solve the perturbative elastostatic equations, we simplify the problem by requiring the radial stress at the free boundary to vanish only on average. This simplified BVP, see Figure 4.2A, does not have a unique solution [196]. Nevertheless, we can find a polynomial solution for the simplified BVP from which we find the engineering stress of the exact BVP in closed-form, as will be shown below. As the exact BVP is the sum of a translation and curvature of the upper plate, we take $\gamma_{1}=0$ in the simplified problem for clarity, retaining only the effect of curvature of the upper plate.

For the simplified BVP, the displacement fields $\mathbf{U}^{(n)}\left(\mathbf{W}^{(n)}\right)$ which solve the perturbative form of the elastostatic equations, equation (4.6), are found for each order in terms of polynomials of odd(even) powers in $r$ and even(odd) in $z$, where the degree of the polynomials in $z$ and $r$ are found through trial and error using Mathematica, 
giving

$$
\begin{aligned}
U^{(0)}(r, z) & =-\frac{\gamma_{2} r}{4}\left[\left(\frac{r}{a}\right)^{2}-\frac{4}{3 S^{2}}\left(1-3\left(\frac{z}{h}\right)^{2}\right)\right], \\
W^{(0)}(r, z) & =\gamma_{2} z\left[\left(\frac{r}{a}\right)^{2}-\frac{2}{3 S^{2}}\left(1-\left(\frac{z}{h}\right)^{2}\right)\right], \\
U^{(1)}(r) & =-\frac{\gamma_{2} r}{4}\left(1-2\left(\frac{r}{a}\right)^{2}\right), \\
U^{(n)}(r) & =(-1)^{n} \frac{\gamma_{2} r}{2}\left(1-\left(\frac{r}{a}\right)^{2}\right), \\
W^{(m)}(z) & =(-1)^{m+1} \frac{4 \gamma_{2} z}{3 S^{2}}\left(1-\left(\frac{z}{h}\right)^{2}\right),
\end{aligned}
$$

where $n \geq 2$ and $m \geq 1$ and $S \equiv a / h$ is the aspect ratio. The prescribed vertical displacements at the sample-plate interfaces, $z=0$ and $z=h$, are taken into account by the zeroth order displacement field $\mathbf{U}^{(0)}(r, z)$. Strikingly, the higher order displacement fields are of equal form, alternating only in sign. This can be seen by noting that the force balance equation, equation (4.6), can be written for $n \geq 1$ as

$$
\nabla \times\left(\nabla \times \mathbf{U}^{(n)}\right)+\nabla^{2}\left(\mathbf{U}^{(n)}+\mathbf{U}^{(n-1)}\right)=0,
$$

where the first term vanishes because the radial translation depends only on $r$ and the vertical only on $z$. For $n \geq 1$ each order has vanishing boundary conditions, it then follows from equation (4.13) that their heterogeneous parts will differ in sign, implying only a homogeneous deformation term as possible differences, compare for example $U^{(1)}(r)$ and $U^{(2)}(r)$.

Assuming $\epsilon \equiv 1-2 \nu<1$, with $\nu$ the Poisson ratio, the perturbation expansion of the displacement field in equation (4.4) is convergent, and we find for the full displacement field

$$
\begin{aligned}
U(r, z) & =-\frac{r}{4} \gamma_{2}\left(\frac{\nu}{1-\nu}\left[1-2 \nu+\left(\frac{r}{a}\right)^{2}\right]-\frac{4}{3 S^{2}}\left[1-3\left(\frac{z}{h}\right)^{2}\right]\right), \\
W(r, z) & =\gamma_{2} z\left[\left(\frac{r}{a}\right)^{2}-\frac{2}{3 S^{2}} \frac{\nu}{1-\nu}\left(1-\left(\frac{z}{h}\right)^{2}\right)\right]
\end{aligned}
$$

which, as one checks, solves the elastostatic equations, equation (4.1)-(4.3), for the 
simplified BVP with any $\nu$. For the stress fields, we find

$$
\begin{aligned}
\sigma_{r r} & =-\gamma_{2} E\left[\frac{\nu}{4\left(1-\nu^{2}\right)}\left(1-\left(\frac{r}{a}\right)^{2}\right)-\frac{1}{3 S^{2}(1-\nu)}\left(1-3\left(\frac{z}{h}\right)^{2}\right)\right] \\
\sigma_{\theta \theta} & =-\gamma_{2} E\left[\frac{\nu}{4\left(1-\nu^{2}\right)}\left(1-3\left(\frac{r}{a}\right)^{2}\right)-\frac{1}{3 S^{2}(1-\nu)}\left(1-3\left(\frac{z}{h}\right)^{2}\right)\right] \\
\sigma_{z z} & =\gamma_{2} \frac{E}{1-\nu^{2}}\left[\left(\frac{r}{a}\right)^{2}-\frac{\nu^{2}}{2}\right] \\
\sigma_{r z} & =0
\end{aligned}
$$

where $E$ is the Young's modulus. In the limit of large aspect ratio, i.e., in the limit of a plate cylinder with $S \gg 1$, the radial stress-free boundary condition in the simplified BVP is satisfied pointwise, and the solution becomes exact and unique.

In principle, we can find the engineering stress from equation (4.18). As the solution solves the simplified BVP, however, it is not generally unique. Therefore, we find the solution to the exact BVP by adding the corrective solution $\mathbf{U}^{\mathrm{cor}}(r, z)$ to the solution of the simplified BVP [193], see Figure 4.2A for the boundary value problem appurtanent to the corrective solution and Figure 4.2B for the relation between the exact, the simplified and the corrective solution. This corrective solution cancels the erroneous radial tractions acting on the free boundary in the simplified BVP, see equation (4.16). The corrective solution does not add to the engineering stress, however, as will be shown below.

For any radial and tangential stress distribution at the curved boundary of a frictionless cylinder that are, respectively, symmetric and antisymmetric around $z=0$, the corrective solution satisfying the BVP given in Figure 4.2 can be written as

$$
\mathbf{U}^{\mathrm{cor}}(r, z)=\sum_{n=0}^{\infty}\left(C_{1, n} \mathbf{U}_{n}^{\mathrm{cor}, 1}(r, z)+C_{2, n} \mathbf{U}_{n}^{\mathrm{cor}, 2}(r, z)\right)
$$

with $C_{1, n}$ and $C_{2, n}$ arbitrary constants, and

$$
\begin{aligned}
U_{n}^{\mathrm{cor}, 1}(r, z) & =-I_{1}\left(\beta_{n} r\right) \cos \left(\beta_{n} z\right), \\
W_{n}^{\mathrm{cor}, 1}(r, z) & =I_{0}\left(\beta_{n} r\right) \sin \left(\beta_{n} z\right), \\
U_{n}^{\mathrm{cor}, 2}(r, z) & =\left(4(1-\nu) I_{1}\left(\beta_{n} r\right)-\beta_{n} r I_{0}\left(\beta_{n} r\right)\right) \cos \left(\beta_{n} z\right), \\
W_{n}^{\mathrm{cor}, 2}(r, z) & =\beta_{n} r I_{1}\left(\beta_{n} r\right) \sin \left(\beta_{n} z\right),
\end{aligned}
$$


where $\beta_{n} \equiv \pi n / h$.

Suppose the radial $f(z)$ and tangential $g(z)$ tractions at the curved boundary can be expanded as $f(z)=\sum_{n=1}^{\infty} f_{n} \cos \left(\beta_{n} z\right)$ and $g(z)=\sum_{n=1}^{\infty} g_{n} \sin \left(\beta_{n} z\right)$. Solving for $C_{1, n}$ and $C_{2, n}$, we find for the engineering stress induced by the tractions

$$
\left.\bar{\sigma}_{z z}^{\mathrm{cor}} \equiv \frac{1}{\pi a^{2}} \int_{0}^{a} \mathrm{~d} r 2 \pi r \sigma_{z z}^{\mathrm{cor}}\right|_{z=h} \propto \sum_{n=1}^{\infty}(-1)^{n} \frac{g_{n} h}{n \pi a},
$$

which shows that only with nonzero tangential stresses at the free boundary, the corrective solution adds to the engineering stress. To correct the polynomial solution to the simplified BVP, we set $f(z)=-\left.\sigma_{r r}\right|_{r=a}$ and $g(z)=-\left.\sigma_{r z}\right|_{r=a}$, with $\sigma_{r r}$ and $\sigma_{r z}$ from equation (4.16) and (4.19). Because the tangential stress at the free boundary in the polynomial solution vanishes, however, we have $g(z)=0$, and we observe from equation (4.25) that the corrective solution gives zero contribution to the engineering stress.

Finally, to obtain the solution to the exact BVP, see Figure 4.2, we add to the solution of the simplified BVP both the corrective solution and that of a translation of the upper plate, i.e., that of plate-plate compression with engineering strain $\gamma_{1}$. From this exact solution, we find the engineering stress as

$$
\frac{F}{\pi a^{2}}=E \gamma_{\mathrm{eq}}
$$

where $\gamma_{\text {eq }} \equiv \gamma_{1}-\gamma_{2} / 2$ is the equivalent strain. Equation (4.26) holds for any aspect and Poisson ratio, provided there is full contact between the spherical surface and the upper cylinder end. The equivalent strain equals the engineering strain in a hypothetical plate-plate compression experiment where the upper flat plate displaces the same volume as the curved plate in a plate-sphere compression experiment. This result is to be expected for any long prismatic bar with end loading [204], but is here found to hold for a frictionless cylinder of any aspect ratio. This result allows for straightforward interpretation of measured stress-strain curves in plate-sphere compression experiments on any linear elastic homogeneous and isotropic material, similar to plate-plate compression. 


\subsection{Conclusions and discussion}

Using a novel perturbative approach we obtain the exact solution for the plate-sphere compression of a frictionless cylindrical sample by combining a full field polynomial solution of the displacement field with the corrective solution. The former provides the exact closed-form expression for the engineering stress for cylindrical samples of any aspect ratio. In the limit of high aspect ratio the polynomial solution for the displacement field becomes exact. Our solution enables plate-sphere compression as a method to determine the Young's modulus, with the possible benefit of avoiding misalignment problems in the experimental setup.

For a long cylinder, the result we found for the load is exactly what one would expect from standard methods [204], since in the plane strain regime the equivalent strain should determine the vertical stress by Saint Venant's principle. It is striking, however, that the load is exact for all aspect ratio's.

In the plate-sphere compression method we propose, the problem of geometrical misalignment is replaced by the need to move the tip of a spherically curved plate in line with the symmetry axis of the cylinder. In principle, this is easy to solve as the sample can be positioned accurately in line with the tip of the spherical surface. A possible source of error which remains, however, is that the tip of the surface could move misaligned with the axis of the cylinder. Future work should examine the influence of this misalignment on the deformation of the sample.

For large curvatures of the upper surface the vertical normal stress at the sample-plate required for full contact can be positive, implying adhesive forces to be present. For many materials no significant adhesive forces are present. Practically, the theoretical compression tests which need adhesive forces are easily excluded, however, as a nonadhesive material will not make full contact with the spherical surface.

We hope our perturbative approach to be fruitful in many problems where an exact solution is lacking. By finding a simple approximate solution, as illustrated here, some properties of the system can yet be found exactly. As noted above, our perturbative approach reminds somewhat of the recursive approach to the three-dimensional linear elastic problem of a long prismatic bar subjected to arbitrary polynomial tractions on its lateral surfaces [201], though this approach holds only for relatively long bars. Relations between our perturbative approach and the recursive approach could be a 
subject for further research. The conventional analysis of the perturbative equations we obtained involves the use of vector field decomposition and the search for appropriate scalar and vector potential functions. This kind of analysis is a matter for future research.

We assumed the material to be linear elastic, which can be a good approximation of a real material up to $10 \%$ engineering strain. Therefore, given that the erroneous tractions scale as $1 / S^{2}$, our polynomial solution for the displacement field is accurate for disk-like samples with aspect ratio $S \gtrsim 10$, provided friction between the cylinder and the compression surfaces is negligible.

\subsection{Appendix}

\subsubsection{Standard problems with perturbation theory}

For illustration, we treat three standard problems within our perturbative approach.

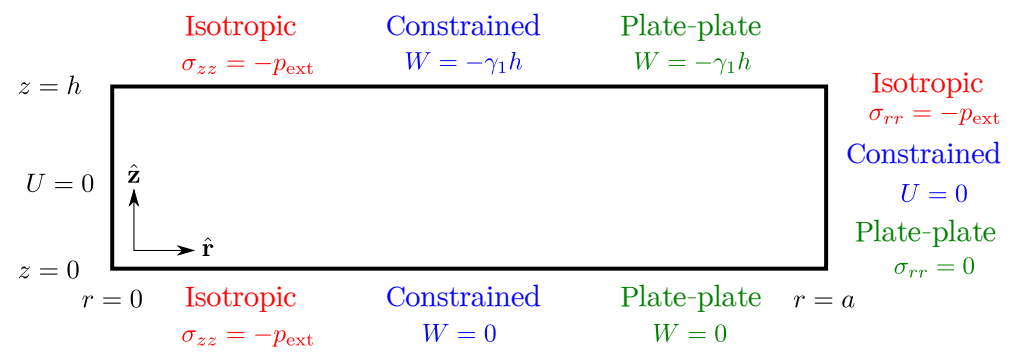

Figure 4.3: Illustration of the boundary value problem for the displacement field $\mathbf{U}(r, z)=$ $U(r, z) \hat{\mathbf{r}}+W(r, z) \hat{\mathbf{z}}$ of a cylindrical sample in the standard problems of isotropic (red), constrained (blue) and plate-plate (green) compression. All problems have vanishing tangential tractions at each boundary $\sigma_{r z}=G\left(\partial_{z} U+\partial_{r} W\right)=0$.

\section{Isotropic compression}

For a cylinder under some uniform external hydrostatic pressure $p_{\text {ext }}$, see Figure 4.3, at all sides without any tangential tractions acting at the boundaries, the solution of 
the force balance equations can be found as

$$
\begin{array}{ll}
U^{(0)}=-\gamma r, & W^{(0)}=-\gamma z, \\
U^{(n)}=0, & W^{(n)}=0,
\end{array}
$$

where $n \geq 1$. Only the zeroth and first order contribute to the stresses which are uniform throughout the body. From, say, the radial $\mathrm{BC} \sigma_{r r}=-p_{\text {ext }}$ we then find $\gamma=p_{\text {ext }} / 3 K$.

\section{Constrained compression}

For a frictionless constrained cylinder under a load $F$, see Figure 4.3, the force balance equations of motion can be solved by taking

$$
\begin{array}{ll}
U^{(0)}=0, & W^{(0)}=-\gamma_{1} z, \\
U^{(n)}=0, & W^{(n)}=0,
\end{array}
$$

where $n \geq 1$. From the stress BC's, we find the zeroth order and first order to contribute to the vertical and the radial stress, implying $\gamma_{1}=F / M \pi a^{2}$ and $\sigma_{r r}=$ $-\gamma_{1} \Lambda$, with $M \equiv K+4 G / 3$ the longitudinal modulus and $\Lambda \equiv K-2 G / 3$ Lamé's first parameter.

\section{Plate-plate compression}

For a cylinder under plate-plate compression, see Figure 4.3, under an engineering strain $\gamma_{1}$, the solution of the force balance equations can be found as

$$
\begin{array}{ll}
U^{(0)}=\gamma_{1} r / 2, & W^{(0)}=-\gamma_{1} z, \\
U^{(1)}=-\gamma_{1} r / 2, & W^{(1)}=0, \\
U^{(n)}=0, & W^{(n)}=0,
\end{array}
$$

where $n \geq 2$. Only the first order of the radial stress is nonzero. At zeroth order, there is zero contribution to the vertical stress, but it receives nonzero contributions in first and second order, giving rise to the Young's modulus $E=9 K G /(3 K+G)$. 


\subsubsection{Classical derivation of the polynomial solution}

The solution to the simplified BVP, equation (4.14) and (4.15), can alternatively be derived using the polynomial potential functions $\phi_{n}^{A}$ and $\phi_{m}^{B}$ [205]

$$
\begin{aligned}
& \phi_{n}^{A}(r, z)=\left(r^{2}+z^{2}\right)^{n / 2} P_{n}\left(\frac{z}{\sqrt{r^{2}+z^{2}}}\right), \\
& \phi_{m}^{B}(r, z)=\left(r^{2}+z^{2}\right) \phi_{m-2}^{A}(r, z),
\end{aligned}
$$

where $n \geq 0$ and $m \geq 2$ and $P_{n}$ is the $n$-th Legendre polynomial. From the polynomial potential functions four linearly independent solutions $f_{i}(r, z)$ can be formed which separately satisfy $\sigma_{r z}=0$ for any value of $r$ and $z$

$$
\begin{aligned}
& f_{1}(r, z)=\phi_{3}^{A}(r, z), \\
& f_{2}(r, z)=\phi_{3}^{B}(r, z), \\
& f_{3}(r, z)=\frac{\phi_{4}^{A}(r, z)}{12}+\frac{\phi_{4}^{B}(r, z)}{2(8-7 \nu)}, \\
& f_{4}(r, z)=\frac{\phi_{5}^{A}(r, z)}{60}+\frac{\phi_{5}^{B}(r, z)}{6(8-9 \nu)},
\end{aligned}
$$

giving as a general solution $f^{\text {tot }}(r, z)=C_{1} f_{1}(r, z)+C_{2} f_{2}(r, z)+C_{3} f_{3}(r, z)+$ $C_{4} f_{4}(r, z)$, with $C_{i}$ arbitrary constants. The displacement field $\mathbf{U}(r, z)=U(r, z) \hat{\mathbf{r}}+$ $W(r, z) \hat{\mathbf{z}}$ can be found as $U=-\partial_{r} \partial_{z} f^{\text {tot }} / 2 G$ and $W=\left(2(1-\nu) \nabla^{2}-\partial_{z}^{2}\right) f^{\text {tot }} / 2 G$, with $G$ the shear modulus and $\nabla^{2}$ the Laplacian [205]. Next, the prescribed vertical displacements at the cylinder ends, $W=h \gamma_{2}(r / a)^{2}$ at $z=h$ and $W=0$ at $z=0$, are uniquely satisfied by setting

$$
\begin{aligned}
C_{2} & =\frac{3 C_{1}}{7-10 \nu}-\frac{2}{3} \gamma_{2} G\left(\frac{h}{a}\right)^{2} \frac{\nu}{(1-\nu)(7-10 \nu)}, \\
C_{3} & =0 \\
C_{4} & =\frac{4}{9} \gamma_{2} G \frac{1}{a^{2}} \frac{9 \nu-8}{1-\nu} .
\end{aligned}
$$

Finally, requiring the average radial tractions to vanish at the free boundary implies

$$
C_{1}=-\gamma_{2} G \frac{3(7-10 \nu) \nu-4(h / a)^{2}(7-5 \nu)}{90(1-\nu)}
$$


and

$$
C_{2}=\gamma_{2} G \frac{4(h / a)^{2}-3 \nu}{30(1-\nu)} .
$$

With the arbitrary constants $C_{i}$ fixed, we find the solution for the displacement field in equation (4.14) and (4.15). 


\section{Chapter 5}

\section{Scoop syneresis}

The results of this chapter are contained in ${ }^{1}$ :

Q. Wu, M.T.J.J.M. Punter, T.E. Kodger, L. Arnaudov, B.M. Mulder, S. Stoyanov \& J. van der Gucht. "Gravity-driven syneresis in model low fat mayonnaise". In: Soft Matter 15.46 (Nov. 2019), pp. 9474-9481.

\footnotetext{
${ }^{1}$ The experiments reviewed in this chapter have been designed and carried out by Qimeng Wu, T.E. Kodger \& J. van der Gucht from Wageningen University (The Netherlands) and L. Arnaudov \& S. Stoyanov from Unilever B.V.
} 


\section{Abstract}

In the food industry, low fat products are often enriched with polymer-forming ingredients, e.g., starch, to obtain similar mechanical properties and mouth feel as full fat products. Syneresis, i.e., densification stemming from fluid expulsion, is a common problem associated with low fat products. To obtain insight in the governing mechanism of syneresis in low fat mayonnaise, we study a model system composed of emulsified oil droplets with heated starch in the aqueous phase. In syneresis experiments, the fluid expulsion was measured and we find the rate of expulsion to be proportional to the hydrostatic pressure. We formulate a two-cylinders model which takes into account the geometry of the experimental setup by separating it into a cylinder with high and a cylinder with low fluid velocity. First, the measured fluid expulsion is explained by assuming filter paper clogging and the subsequent buildup of a cake of densified mayonnaise. Through additional experiments, filter paper clogging is shown to be negligible, however, and instead an oil droplet-starch network is hypothesized to govern the expulsion of fluid. Regarding the network as a quasi-static porous medium of oil droplets, and using the two-cylinders model, the expulsion of fluid is explained as a hydrostatically driven flow of fluid through a bed of packed oil droplets. 


\subsection{Introduction}

A known and poorly understood problem for low fat mayonnaise is its lack of stability. It suffers from the expulsion of watery fluid after a first spoon has been taken from the mayonnaise jar, implying reduced attractiveness for consumers. Remarkably, the expulsion does not occur in the absence of spooning, suggesting, for instance, a role for the height difference that spooning induces. Therefore, we pose the question, what physical mechanism governs fluid expulsion from low fat mayonnaise?

Fluid expulsion has been measured in a model system for low fat mayonnaise: a starch paste, combined with typical mayonnaise ingredients, with oil droplets dispersed throughout. Searching for the physical mechanism that governs the expulsion, we first hypothesize that a fringe effect, i.e., clogging of the filter paper used in the experimental setup, determines the fluid expulsion. This investigation serves to put the experimental results under scrutiny. As the theoretical results for filter clogging compare well to experiments, it seems a possible explanation of the observed fluid expulsion. Additional experiments, however, exclude filter paper clogging as an explanatory mechanism for fluid expulsion. Next, the measured fluid expulsion is hypothesized to arise from the local yielding and reforming of both an oil droplet and a starch network. It is argued that this double network can act as a semi-static porous medium for fluid to flow through.

Below, after introducing the experimental setup, materials and results, we model the experimental geometry with a two-cylinders model. Thereafter, we introduce and argue for the filter paper clogging hypothesis, implement it, and compare the results to experiments. Next, the validation experiments are introduced which show no filter paper clogging to be present. Finally, the fluid expulsion is explained as a hydrostatically driven syneresis of the double network in the model low fat mayonnaise.

\subsection{Syneresis experiments: materials, methods and re- sults}

The model low fat mayonnaise is composed of $52 \mathrm{v} \%$ of sunflower oil and $48 \mathrm{v} \%$ of aqueous phase. Sunflower oil is added to the aqueous phase in the form of emulsified oil droplets using a Silverson LSM-A Laboratory Mixer with Emulsor Screens 
$\mathrm{A}$

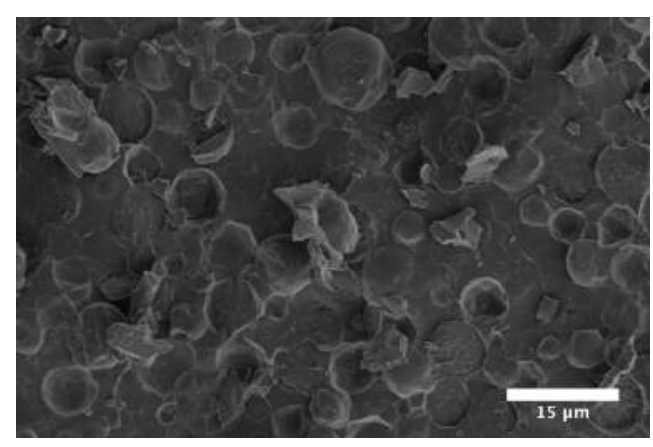

$\mathrm{B}$

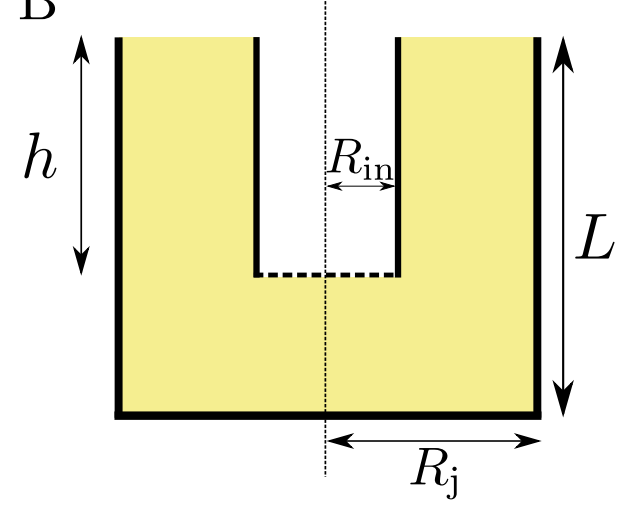

Figure 5.1: A) Scanning electron microscopy image of the model low fat mayonnaise. Oil droplets are approximately spherical due to the relatively low packing fraction and have radii in the order of micrometers. B) Schematic of the container setup in which the expulsion experiments on the model low fat mayonnaise (yellow) have been conducted. The radius of the jar is $R_{\mathrm{j}}=3.14 \mathrm{~cm}$ and the radius of the inner tube is $R_{\text {in }}=1 \mathrm{~cm}$. The vertical dashed line gives the axis of cylindrical symmetry and the horizontal dashed line denotes the filter paper at the bottom of the tube. The total height of the mayonnaise in the jar is $L=4.8 \mathrm{~cm}$ and the height of the inner tube, setting the height of the mayonnaise column above the filter paper, is $h$.

at $5800 \mathrm{rpm}$. Oil droplets are produced whose radius is in the order of micrometers, see Figure 5.1A. To have the model low fat mayonnaise be reminiscent of real low fat mayonnaise, the aqueous phase consists of $4 \mathrm{wt} \%$ native rice starch, $7 \mathrm{wt} \%$ of egg yolk, $5 \mathrm{wt} \%$ of vinegar, $2.8 \mathrm{wt} \%$ of $\mathrm{NaCl}$, and is prepared as follows. First, the rice starch is suspended in Milli-Q water and gently stirred for 5 minutes, thereby avoiding sedimentation and/or agglomeration, at a temperature of $90{ }^{\circ} \mathrm{C}$, while compensating for evaporation. Afterwards, the suspension is cooled to $50{ }^{\circ} \mathrm{C}$ and the egg yolk, the vinegar and the $\mathrm{NaCl}$ are added.

In the validation experiments, see section 5.5, the syneresis of a starch paste is compared to syneresis of the model low fat mayonnaise. To obtain a starch paste, a $4 \mathrm{wt} \%$ native starch suspension is heated, as described above, albeit no other ingredients are added.

It is known that starch, when heated above the gelation temperature, forms a turbid viscoelastic paste consisting of an amylose network with swollen starch granules acting as a filler [206, 207]. In section 5.6, it is shown through rheology experiments that 


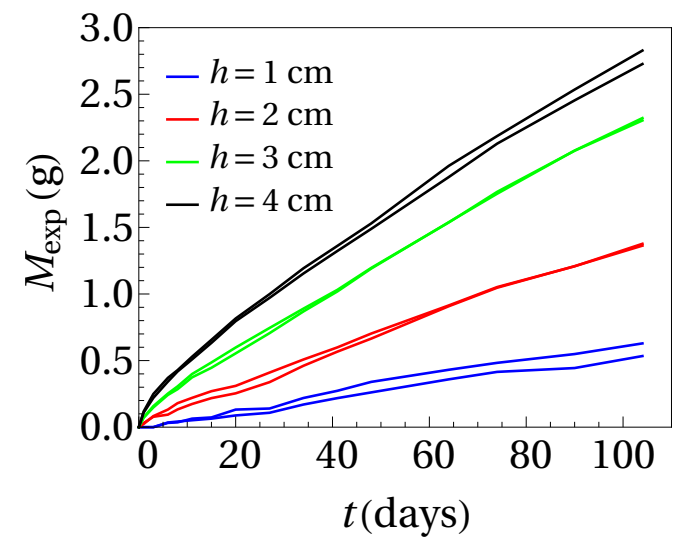

(A)

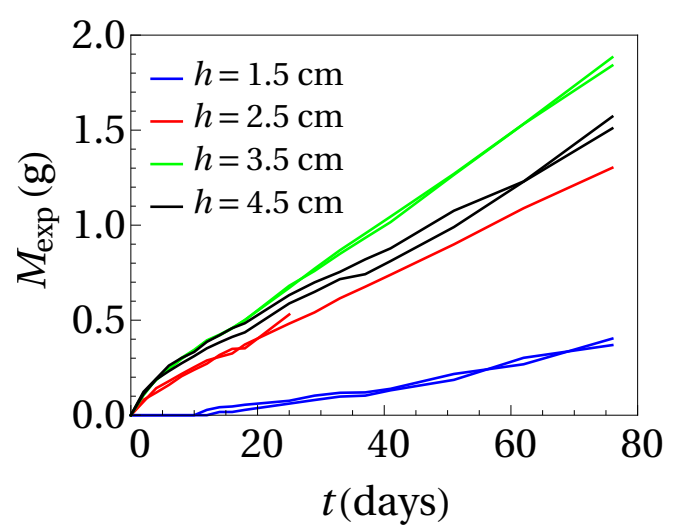

(B)

Figure 5.2: The mass of expelled fluid $M_{\mathrm{ex}}$ into the inner tube from the model low fat mayonnaise for integer values (A) and half-integer values $(\mathbf{B})$ of the height of the inner tube $h$ as a function of time $t$, see Figure 5.1 for the experimental setup. Each experiment has been performed twice.

the starch paste indeed has the rheological characteristics of a complex viscoelastic fluid, see Figure 5.10. Interestingly, the aqueous phase does not seem to have a yield stress, see Figure 5.5, implying the egg yolk, vinegar and $\mathrm{NaCl}$ to alter the rheological characteristics of the starch paste.

The syneresis experiments on model low fat mayonnaise have been conducted in a container setup, see Figure 5.1B. In this setup, a mayonnaise filled inner tube with filter paper at the bottom is located inside a volume of mayonnaise in a cylindrical jar, and is left for a week before initiation of a syneresis experiment. To initiate the experiment, the mayonnaise in the tube is aspirated from the open end. As soon as the tube is filled with air, a hydrostatic pressure difference is established between the inside of the tube and the mayonnaise just below the bottom end of the tube, thereby simulating a scoop taken from a real-life mayonnaise. It is observed that over time fluid, consisting of a combination of water and dissolved starch components, is expelled from the mayonnaise without any oil, see Figure 5.2 for the expelled mass $M_{\mathrm{ex}}$ as a function of time $t$. In the first few weeks the rate of fluid expulsion decreases, after which it seems to become approximately constant.

We determine the average rate of fluid expulsion $\bar{r}$ by fitting $M_{\mathrm{ex}}=\bar{r} t$ to the measurements. The fitted expulsion rates $\bar{r}$ increase proportional to $h$ for $h \leq 4 \mathrm{~cm}$, 


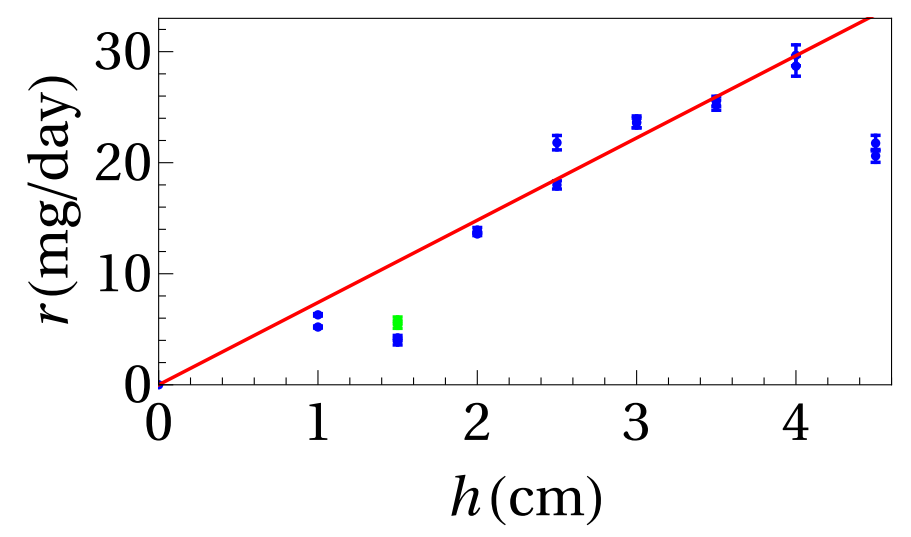

Figure 5.3: The fitted average rate of fluid expulsion $\bar{r}=M_{\mathrm{ex}} / t$ for the syneresis experiments in Figure 5.2 (blue dots), the error bars represent the fit uncertainties. Apart from the $h=1.5 \mathrm{~cm}$ and $h=4.5 \mathrm{~cm}$ experiments, the average expulsion rate seems to scale with the height of the inner tube $\bar{r} \propto h$. A linear fit of the expulsion rates (red line) gives $\bar{r} / h=7.4(0.1) \mathrm{mg} \mathrm{day}^{-1} \mathrm{~cm}^{-1}$, with the uncertainty in brackets. The green dots give the average expulsion rate for the $h=1.5 \mathrm{~cm}$ experiments when ignoring the lag phase.

see Figure 5.3. Ignoring the $h=1.5 \mathrm{~cm}$ experiments, we fit the line $r=a h$ to the inferred expulsion rates for $h \leq 4 \mathrm{~cm}$, giving $a=7.4(0.1) \mathrm{mg} \mathrm{day}^{-1} \mathrm{~cm}^{-1}$ with the uncertainty in brackets, see the red line in Figure 5.3. The proportionality of the expulsion rates with $h$ suggests the syneresis process to be driven by the hydrostatic pressure $\Delta p=\rho g h$ of the mayonnaise column around the inner tube above the level of the filter paper, with $\rho$ the density of the low fat mayonnaise and $g$ the gravitational acceleration.

The $h=1.5 \mathrm{~cm}$ experiments seem to break the trend $\bar{r} \propto h$, however. This could be connected to the fact that in these experiments there is a lag time for the fluid expulsion to initiate. This lag time could be caused by an energy barrier which needs to be overcome before fluid expulsion can initiate, or by some slow time-dependent process. Ignoring the lag phase and refitting the average rate of fluid expulsion $\bar{r}$ with the line $M_{\mathrm{ex}}=\bar{r} t+b$, taking $b$ as a fit parameter, the $h=1.5 \mathrm{~cm}$ experiments still seem to break the trend, see the green datapoints in Figure 5.3.

For $h>4 \mathrm{~cm}$ the expulsion rate decreases. Possibly, this is because the total height of the mayonnaise in each experiment is $L=4.8 \mathrm{~cm}$, see Figure $5.1 \mathrm{~B}$, causing the bottom of the jar to generate boundary effects which influence the fluid flow if $h$ becomes too large. If so, the fluid flow would be negligible more than about $1 \mathrm{~cm}$ below 


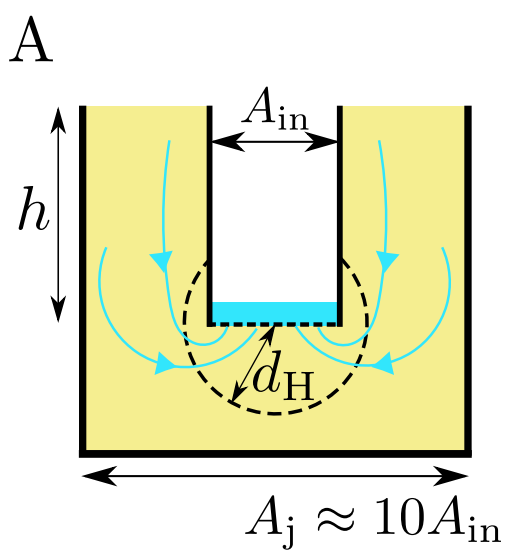

$\mathrm{B}$

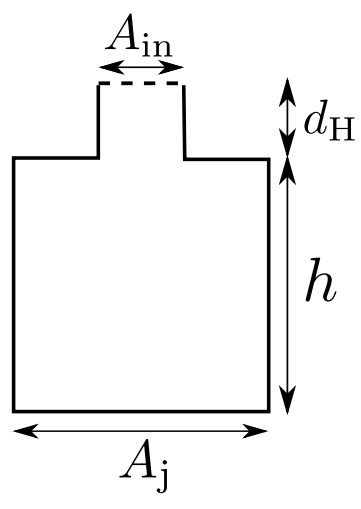

Figure 5.4: A) As fluid flows out of model low fat mayonnaise, a region of high fluid velocity, with characteristic size $d_{\mathrm{H}}$, and a region of low fluid velocity, whose characteristic size equals the height of the mayonnaise in the jar $h$. B) The two-cylinders model abstracts the 3D fluid flow of the experimental geometry as a 1D flow through two connected cylinders with unequal cross-section. The high-velocity cylinder ends at the filter paper of surface area $A_{\text {in }}$, thereby setting its cross-sectional area. The low-velocity cylinder has cross-sectional area $A_{\mathrm{j}}$, with $A_{\mathrm{j}}$ the end surface area of the jar.

the filter paper. Assuming the fluid flow to be isotropically concentrated around the filter paper, this suggests that for $h \leq 4 \mathrm{~cm}$ the entire jar has negligible influence on the fluid flow, because the radius of the jar $R_{\mathrm{j}}=3.14 \mathrm{~cm}$ while the radius of the inner tube is $R_{\text {in }}=1 \mathrm{~cm}$. Given these observations, we model the geometry of the experimental setup in the next section as two connected cylinders, one with high fluid velocity near the filter paper, and one with low fluid velocity far away from the filter paper.

\subsection{Two-cylinders model}

Before considering a specific mechanism governing the syneresis, we model the geometry of the experimental setup, because this generally determines the flow of fluid through the mayonnaise. The experimental setup has axial symmetry, and is parametrized by the height of the jar $L=4.8 \mathrm{~cm}$, the radius of the inner tube $R_{\text {in }}=1 \mathrm{~cm}$, the radius of the jar $R_{\mathrm{j}}=3.14 \mathrm{~cm}$ and the height of the inner tube 
$h$, which is varied in the experiments, see Figure 5.1B. Independent of the height of the inner tube, however, the fluid has to flow from the bulk of the mayonnaise through the filter paper into the inner tube, see Figure 5.4A. As the end area of the filter paper is $A_{\text {in }}=\pi R_{\text {in }}^{2}$ and the end area of the jar is $A_{\mathrm{j}}=\pi R_{\mathrm{j}}^{2}$, the cross-section of the fluid flow should decrease when fluid flows from, e.g., the top of the mayonnaise in the jar towards the filter paper. As explained in section 5.2, the mayonnaise within about 1 $\mathrm{cm}$ from the filter paper seems to significantly influence the rate of fluid expulsion. Therefore, we model the fluid flow as a one-dimensional flow through a cylinder with high fluid velocity and a cylinder with low fluid velocity, see Figure 5.4B. The lowvelocity cylinder models the mayonnaise in the jar far away from the filter paper, has end area $A_{\mathrm{j}}$, its length equals the height of the inner tube $h$, and is with one end connected to the high-velocity cylinder. We take the length to be $h$ because the fluid above the level of the filter paper is driven by gravity to flow into the inner tube. The high-velocity cylinder connects the low-velocity cylinder with the filter paper, has end area $A_{\text {in }}$ and length $d_{\mathrm{H}}$. We expect $d_{\mathrm{H}}$ to be dependent on the radius of the inner tube $R_{\text {in }}$, because it determines over what distance the fluid should flow horizontally, but not on $L$ and $R_{\mathrm{j}}$ for $h \leq 4 \mathrm{~cm}$, as argued for in the previous section. Due to mass conservation, the fluid flow velocities in the two cylinders are related by

$$
v_{\mathrm{L}} A_{\mathrm{j}}=v_{\mathrm{H}} A_{\text {in }},
$$

where $v_{\mathrm{L}}$ is the fluid velocity in the low-velocity cylinder and $v_{\mathrm{H}}$ is the fluid velocity in the high-velocity cylinder. Since $A_{\mathrm{j}} \approx 10 A_{\mathrm{in}}$, the low fluid velocity $v_{\mathrm{L}}$ is about an order of magnitude smaller than the high fluid velocity $v_{\mathrm{H}}$. With this model for the experimental geometry, the mechanisms governing syneresis can be explicated, as will be done in section 5.4 and 5.6.

\subsection{Filter paper clogging}

In this section we argue for the hypothesis that the observed fluid expulsion in the syneresis experiment, see Figure 5.2, can be explained by clogging of the filter paper with oil droplets, and that the expulsion of fluid causes the mayonnaise to compactify into a cake of close-packed oil droplets, such that the resistance against fluid outflow increases over time. First, we consider how a clogged filter paper can set the initial outflow of fluid from the mayonnaise. Second, with the two-cylinders model the 
fluid outflow during compactification of mayonnaise is calculated. Finally, fitting the results for the fluid outflow to the available experimental results, we find credible values for the two fit parameters: the length of the high-velocity cylinder $d_{\mathrm{H}}$, see section 5.3, and the influence of the clogged filter paper on the outflow of fluid. If the filter paper is clogged with oil droplets, the majority of the hydrostatic pressure is found to be dissipated in the clogged filter paper throughout the experiment.

\subsubsection{Clogging hypothesis}

The model low fat mayonnaise has a yield stress in the order of 0.1 Pa, see Figure 5.5, implying it has weak solid-like properties. As the hydrostatic pressure differences are typically in the order of $100 \mathrm{~Pa}$, the model low fat mayonnaise is expected to flow as a fluid. Therefore, the model low fat mayonnaise should flow as a whole through the filter paper once the hydrostatic pressure difference is present. Since the filter paper is semi-permeable, however, the oil droplets, combined with swollen starch granules, can not pass and could clog the filter paper instead.

The rate of fluid outflow from the model low fat mayonnaise initially decreases, approaching an approximately constant value after at most a few weeks, see Figure 5.2. This suggests that the resistance against fluid outflow initially increases, which would be the case if a cake of close-packed oil droplets forms beneath the filter paper by compactification of the mayonnaise.

Taking into account the above observations, we hypothesize that as soon as the hydrostatic pressure difference is actuated, the mayonnaise is pushed through the filter paper and clogs it with oil droplets and insoluble starch components. Therefore, the permeability of the filter paper is reduced drastically and the initial rate of fluid outflow is set. Upon the expulsion of fluid the mayonnaise near the filter paper compactifies into a cake until the oil droplets are at random close packing. Fluid outflow causes this compactified layer to grow throughout the mayonnaise. The larger this layer, the more resistance against fluid flow, thereby decreasing the rate of fluid expulsion over time.

In section 5.2 the average rate of fluid expulsion $\bar{r}$ was shown to increase proportional to the hydrostatic pressure of the mayonnaise above the level of the filter paper $\Delta p=\rho g h$. In Darcy flow, the average fluid velocity through a porous medium is 


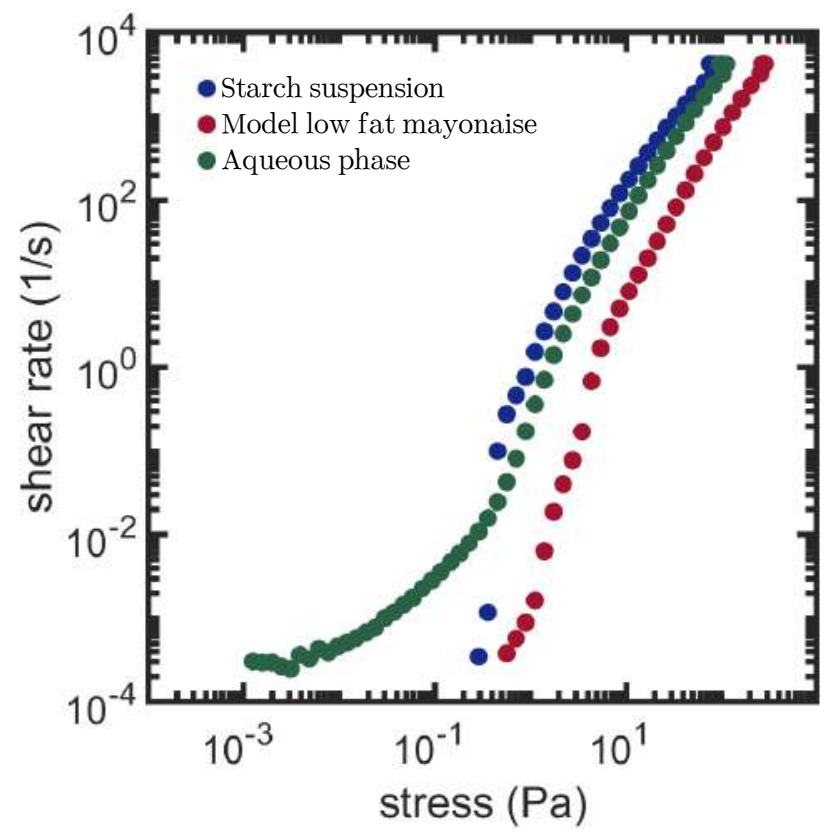

Figure 5.5: Rheology measurements of the required stress to establish a given shear rate for the model low fat mayonnaise (red), the starch paste (blue), and the aqueous phase used to prepare the mayonnaise (green). The starch paste and model low fat mayonnaise have a yield stress which is in the order of $0.1 \mathrm{~Pa}$.

proportional to the pressure change, suggesting the mayonnaise should be regarded as a porous medium.

Assuming the clogged filter paper to obey Darcy's law with filter paper permeability $k_{\mathrm{f}}$, thickness $d_{\mathrm{f}}$ and dynamic viscosity of the expelled fluid $\eta$, we obtain for the initial superficial velocity of fluid $v_{\text {i }}$ through the filter paper, i.e., the fluid velocity averaged over the total surface area of the filter paper,

$$
v_{\mathrm{i}}=\frac{k_{\mathrm{f}}}{\eta} \frac{\rho g h}{d_{\mathrm{f}}},
$$

where $\rho g h$ is the hydrostatic pressure difference induced by the mayonnaise column of height $h$, with $\rho \approx 10^{3} \mathrm{~kg} / \mathrm{m}^{3}$ the density of the fluid and the mayonnaise which we take to be approximately equal because we observe no creaming, and $g=9.81 \mathrm{~m} / \mathrm{s}^{2}$ the gravitational acceleration constant. From measurements, we can infer what the initial average fluid velocity $v_{\mathrm{i}}$ is, because we know the initial expulsion rate $r_{0}$ of fluid from the mayonnaise from Figure 5.2. The expulsion rate 
obeys at all times

$$
r=v \rho A_{\text {in }}
$$

with $A_{\text {in }}=\pi R_{\text {in }}^{2}$ the surface area of the filter paper, see Figure 5.1, with $R_{\text {in }}=1 \mathrm{~cm}$

From Figure 5.2 we find the initial expulsion rate for, e.g., $h=3 \mathrm{~cm}$ to be about $r_{0}=70 \mathrm{mg} \mathrm{day}^{-1}$ at the start of the experiment, implying the initial fluid expulsion velocity to be $v_{\mathrm{i}}=3 \cdot 10^{-9} \mathrm{~m} \mathrm{~s}^{-1}$ with equation (5.3). We then find $k_{\mathrm{f}} / \eta d_{\mathrm{f}}=$ $9 \cdot 10^{-12} \mathrm{~kg}^{-1} \mathrm{~m}^{2} \mathrm{~s}$. Estimating the dynamic viscosity of expelled fluid to be slightly higher than that of pure water due to soluble starch components, say $\eta \sim 10 \mathrm{mPa} \cdot \mathrm{s}$, and estimating the thickness of the clogged layer in the filter paper to be in the order of ten times the order of magnitude of the average oil droplet radius $R_{\mathrm{oil}}=1 \mu \mathrm{m}$, see Figure 5.1, implying $d_{\mathrm{f}} \sim 10 \mu \mathrm{m}$, we find the permeability of the clogged filter paper to be $k_{\mathrm{f}} \sim 9 \cdot 10^{-19} \mathrm{~nm}^{2}$, suggesting the effective pore size of the clogged filter paper to be of the order of nanometers. The treatment of the clogged filter paper with Darcy's law may be too simplistic, however, as the initial fluid velocity could obey a more general law, e.g., $v_{\mathrm{i}} \propto(\Delta p)^{n}$, with $n$ a positive power law exponent.

As soon as fluid is expelled through the clogged filter paper, oil droplets in the bulk mayonnaise must be compactified until they are randomly close packed due to mass conservation. Therefore, the resistance to fluid outflow grows with time because a growing layer of compactified mayonnaise with permeability $k$ arises. We define the resistance as the ratio of the fluid velocity through the filter paper over the net hydrostatic pressure producing the fluid velocity. To compare the contribution to the resistance of the clogged filter paper with that of the compactification layer, it is convenient to define the effective length of the clogged filter paper $d_{\text {eff. This effective }}$ length equals the length of a hypothetical layer of compactified mayonnaise having the same resistance as the filter paper. Therefore, we define

$$
d_{\text {eff }} \equiv d_{\mathrm{f}} \frac{k}{k_{\mathrm{f}}},
$$

which is useful because of the following. First, the definition of the effective length will simplify the results of section 5.4.2. For example, if a pressure difference $\rho g h$ over the clogged filter paper produces an initial fluid velocity $v_{\mathrm{i}}$, the latter can be written as

$$
v_{\mathrm{i}}=\frac{k}{\eta} \frac{\rho g h}{d_{\mathrm{eff}}},
$$


which allows for easy comparison with fluid flow through compactified bulk mayonnaise. Second, in section 5.4.2, where we treat $d_{\text {eff }}$ as a fitting parameter, its magnitude shows the importance of the filter paper, relative to macroscopically compactified mayonnaise, in determining the measured outflow of fluid.

Given the above estimation of the permeability of the clogged filter paper, we can estimate the effective length of the filter paper as follows. Considering the oil droplets in the compactified mayonnaise as random close packed spheres, the volume fraction of the aqueous phase is $\phi_{\mathrm{aq}}=0.375$, and its permeability $k$ can be estimated with the Kozeny-Carman expression [27]

$$
k=\frac{R_{\mathrm{oil}}^{2}}{180} \frac{\phi_{\mathrm{aq}}^{3}}{\left(1-\phi_{\mathrm{aq}}\right)^{2}},
$$

where $R_{\mathrm{oil}}=1 \mu \mathrm{m}$ is order of magnitude of the radius of the oil droplets. With an estimated fluid viscosity of $\eta \sim 10 \mathrm{mPa} \cdot \mathrm{s}$, we find from equation (5.2) and (5.4) with $v_{\mathrm{i}}=3 \cdot 10^{-9} \mathrm{~m} / \mathrm{s}$ for $h=3 \mathrm{~cm}$, that $d_{\mathrm{eff}} \sim 3 \mathrm{~cm}$, which implies the clogged filter paper to have a similar resistance as a macroscopically compacted mayonnaise layer, since the dimensions of the experimental setup are of the order of centimeters.

The above estimation shows that a clogged filter paper can have a significant influence on the outflow of fluid. In the next section, using the two-cylinders model of section 5.3, we account for the expulsion process with a clogged filter paper and a growing compactification layer.

\subsubsection{Clogging in the two-cylinders model}

In the previous section the clogging hypothesis was introduced. Here, we take into account the growth of a layer of compactified oil droplets in the bulk mayonnaise due to the expulsion of fluid. As introduced in section 5.4, we model the experimental geometry with a two-cylinders model, a high-velocity cylinder representing the mayonnaise close to the filter paper and a low-velocity cylinder representing mayonnaise far away.

As water is expelled from the mayonnaise, the compactification layer starts to grow in the high-velocity cylinder. We model the compactification layer as having a well defined length $y(t)$, which grows from $y=0$ at $t=0$ to the length of the high- 
velocity cylinder $y=d_{\mathrm{H}}$, where all of the mayonnaise in the high-velocity cylinder has been compactified, see Figure 5.6A. Afterwards, the mayonnaise in the lowvelocity cylinder also starts to compactify, such that the layer ultimately grows to a length $y=d_{\mathrm{H}}+h$, see Figure 5.6B. After that the mayonnaise will be compacted further like a porous medium due to the elasticity of the randomly close packed oil droplets. Initially, no significant elastic effects arising from the compression and shear of the network of starch components and oil droplets is expected because the yield stress is negligible and the initial volume fraction of oil droplets is $52 \mathrm{v} \%$, well below random close packing. Due to the expulsion of fluid the oil droplets can become random close packed, however, at which point we assume the bulk modulus to be non-negligible.

By mass conservation, the velocity of fluid in the filter paper and the high-velocity cylinder must be equal because the cross-section is constant, i.e., $v_{\mathrm{f}}=v_{\mathrm{H}}$, with $v_{\mathrm{f}}$ the average fluid velocity through the filter paper and $v_{\mathrm{H}}$ the velocity in the high-velocity cylinder. Moreover, the fluid velocities in the two cylinders are related through equation (5.1). Relative to the undisturbed mayonnaise the volume fraction of the aqueous phase in the compactification layer is lowered with $\Delta \phi \approx 0.1$, for the oil droplet volume fraction goes from its initial value $\phi_{\mathrm{oil}, 0}=0.52$ to random close packing $\phi_{\text {rcp }}=0.62$ [208]. Therefore, the larger the compactification layer, the larger the amount of expelled fluid.

Next, the growth of the compactification layer in the two cylinders is treated separately and explicit expressions for the height of the column of expelled fluid in the inner tube as a function of time are derived.

\section{High velocity cylinder}

After initiation of the syneresis experiment, a compactification layer develops in the mayonnaise close to the filter paper: the high velocity cylinder. If the compactification layer is only present in the high-velocity cylinder, i.e., $0<y \leq d_{\mathrm{H}}$, the hydrostatic pressure generated by the mayonnaise column above the filter paper must be balanced by the hydrostatic pressure of the column of expelled fluid, and by dissipation in both the filter paper and the compactification layer. The mayonnaise column generates a pressure of $p_{\mathrm{g}}=\rho g h$, while the column of expelled fluid in the inner tube generates $p_{\text {in }}=\rho g x(t)$, with $x(t)$ the height of the fluid column in the inner tube. 

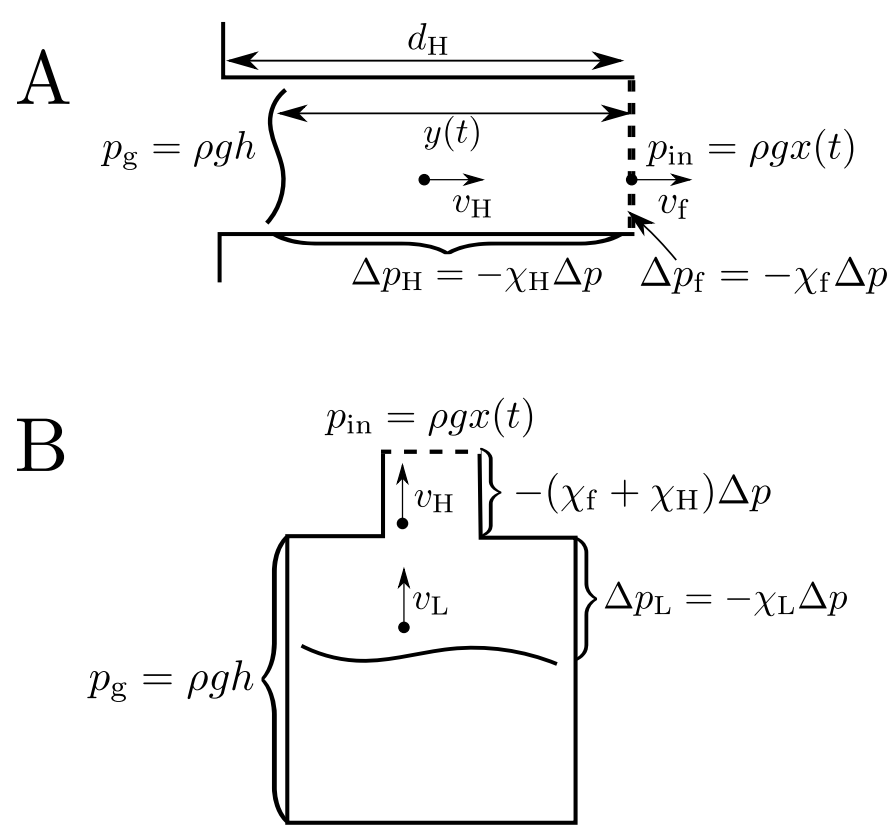

Figure 5.6: A) As the layer of compactified mayonnaise grows in the high-velocity cylinder, $0 \leq y(t)<d_{\mathrm{H}}$, the net hydrostatic pressure $\Delta p \equiv p_{\mathrm{g}}-p_{\text {in }}$ is dissipated in both the compactification layer $\Delta p_{\mathrm{H}}=-\chi_{\mathrm{H}} \Delta p$ and the clogged filter paper $\Delta p_{\mathrm{f}}=-\chi_{f} \Delta p$, with $\chi_{\mathrm{f}}+\chi_{\mathrm{H}}=1$. B) If the compactification layer grows into the low-velocity cylinder, the net hydrostatic pressure $\Delta p$ is dissipated in both the high and low velocity cylinder and the clogged filter paper, with $\chi_{\mathrm{f}}+\chi_{\mathrm{H}}+\chi_{\mathrm{L}}=1$, inducing a fluid velocity $v_{\mathrm{L}}$ in the compactification layer in the low-velocity cylinder.

The net hydrostatic pressure $\Delta p \equiv \rho g(h-x(t))$ is dissipated partly in the filter paper $\Delta p_{\mathrm{f}}=-\chi_{\mathrm{f}} \Delta p<0$ and partly in the compactificaton layer $\Delta p_{\mathrm{H}}=-\chi_{\mathrm{H}} \Delta p<0$, see Figure 5.6A, with $\chi_{\mathrm{H}}+\chi_{\mathrm{f}}=1$, such that $\Delta p+\Delta p_{\mathrm{f}}+\Delta p_{\mathrm{H}}=0$.

The relative dissipation contribution of the filter paper and the compactification layer can be found using mass conservation and Darcy's law. If $y<d_{\mathrm{H}}$, the velocity of fluid through the filter paper $v_{\mathrm{f}}$ and the velocity through the compactification layer in 
the high-velocity cylinder $v_{\mathrm{H}}$ can be written with Darcy's law as

$$
\begin{aligned}
v_{\mathrm{f}} & =\frac{k_{\mathrm{f}}}{\eta} \frac{\chi_{\mathrm{f}} \Delta p}{d_{\mathrm{f}}}, \\
v_{\mathrm{H}} & =\frac{k}{\eta} \frac{\chi_{\mathrm{H}} \Delta p}{y(t)},
\end{aligned}
$$

where $k_{\mathrm{f}}$ and $k$ are the permeability of the filter paper and the compactification layer respectively, $d_{\mathrm{f}}$ is the length of the clogged oil layer in the filter paper, and $\eta$ is the dynamic viscosity of the fluid. Mass conservation implies for a 1D flow with constant cross section that $v_{\mathrm{f}}=v_{\mathrm{H}}$, determining $\chi_{\mathrm{f}}$ as

$$
\chi_{\mathrm{f}}=\frac{d_{\mathrm{eff}}}{d_{\mathrm{eff}}+y(t)},
$$

where we used equation (5.4), and yields for the fluid velocity through the filter paper

$$
v_{\mathrm{f}}=\frac{v_{\mathrm{i}}(1-x(t) / h)}{1+y(t) / d_{\mathrm{eff}}},
$$

where we used equation (5.2). Equation (5.9) shows that as the thickness of the compactification layer grows, a smaller fraction of the hydrostatic pressure is dissipated in the filter paper. Moreover, equation (5.10) shows the fluid velocity through the filter paper to decrease with time both due to antipressure of the expelled fluid and because the total resistance against fluid flow increases upon growth of the compactification layer.

In an infinitesimal time $d t$ the compactification layer grows a distance $d y$, implying with mass conservation that $\Delta \phi_{\mathrm{f}} A_{\text {in }} d y=v_{\mathrm{H}} A_{\text {in }} d t$, i.e., $\Delta \phi_{\mathrm{f}} \dot{y}=v_{\mathrm{H}}$. Therefore, fluid which flows through the compactification layer comes from further compactification of the mayonnaise at the end of the compactification layer. Also, the volume with which the mayonnaise has shrunk due to compactification needs to equal the amount of expelled fluid in the fluid column: $x(t)=\Delta \phi_{\mathrm{f}} y(t)$. Then, we can write equation (5.10), for $0<x \leq x_{\mathrm{H}} \equiv \Delta \phi_{\mathrm{f}} d_{\mathrm{H}}$, as

$$
\dot{x}(t)=\frac{v_{\mathrm{i}}(1-x(t) / h)}{1+x(t) / \Delta \phi_{\mathrm{f}} d_{\mathrm{eff}}},
$$

which can be solved with $x=0$ at $t=0$ to find

$$
\frac{x(t)}{h}=1+\alpha_{\mathrm{H}} W_{0}\left[-\frac{1}{\alpha_{\mathrm{H}}} \exp \left(-\frac{1+t / \tau}{\alpha_{\mathrm{H}}}\right)\right],
$$


where $W_{0}(x)$ is the principal branch of the product logarithm, defined as $z=W_{0}(g)$, if $g=z \exp (z)$ for any real number $z \geq-1$. Moreover, we defined

$$
\alpha_{\mathrm{H}} \equiv 1+\frac{\Delta \phi_{\mathrm{f}} d_{\mathrm{eff}}}{h},
$$

and we defined $\tau \equiv \eta h / \Delta \phi_{\mathrm{f}} k \rho g$ as the typical time scale of fluid expulsion. At time $t=t_{\mathrm{H}}$ the compactification layer comprises the entire high-velocity cylinder, i.e., $y=d_{\mathrm{H}}$ and $x=x_{\mathrm{H}}$, which gives

$$
t_{\mathrm{H}}=\tau\left[\alpha_{\mathrm{H}} \ln \left(\frac{1}{1-x_{\mathrm{H}} / h}\right)-\frac{x_{\mathrm{H}}}{h}\right] .
$$

Summarizing, due to the simplicity of the two-cylinders model we find a definite time at which the compactification layer encompasses the entire high-velocity cylinder. As argued in section 5.2 and 5.3, we expect $d_{\mathrm{H}}$ to be in the order of centimeters, and since $\Delta \phi=0.10$, we expect $x_{\mathrm{H}} \sim 1 \mathrm{~mm}$, implying $x_{\mathrm{H}} \ll h$ for all experiments, and the compactification layer to grow further into the low-velocity cylinder. In the next section, the expulsion of fluid is treated when the compactification layer grows in the low-velocity cylinder.

\section{Low velocity cylinder}

The previous section treated the growth of the compactification layer in the highvelocity cylinder of length $d_{\mathrm{H}}$. Here, we consider its growth in the low-velocity cylinder of length $h$, see Figure 5.6B. When compactification starts to occur in the low-velocity cylinder, the length of the compactification layer satisfies $0<$ $y-d_{\mathrm{H}} \leq h$ and the height of the column of expelled fluid obeys $x(t)=$ $\Delta \phi_{\mathrm{f}}\left[d_{\mathrm{H}}+\left(y(t)-d_{\mathrm{H}}\right) \Lambda\right]$ with $\Lambda \equiv\left(R_{\mathrm{j}} / R_{\text {in }}\right)^{2} \approx 10$, as $R_{\text {in }}=1 \mathrm{~cm}$ and $R_{\mathrm{j}}=$ $3.14 \mathrm{~cm}$. The hydrostatic pressure $p_{\mathrm{g}}=\rho g h$ generated in the mayonnaise column in the low-velocity cylinder is divided between the filter paper $\Delta p_{\mathrm{f}}$, the compactification layer in the high-velocity cylinder $\Delta p_{\mathrm{H}}$, the layer in the low-velocity cylinder $\Delta p_{\mathrm{L}}$ and the hydrostatic pressure of the column of expelled fluid in the inner tube $p_{\text {in }}=\rho g x(t)$, in such a way that the fluid velocities in the cylinders obey mass conservation, see equation (5.1). Force balance requires $p_{\mathrm{g}}+\Delta p_{\mathrm{f}}+\Delta p_{\mathrm{H}}+\Delta p_{\mathrm{L}}=p_{\text {in }}$, and it can be satisfied by taking $\Delta p_{\mathrm{f}}=-\chi_{\mathrm{f}} \Delta p, \Delta p_{\mathrm{H}}=-\chi_{\mathrm{H}} \Delta p$ and $\Delta p_{\mathrm{L}}=-\chi_{\mathrm{L}} \Delta p$, 
with $\Delta p \equiv p_{\mathrm{g}}-p_{\text {in }}=\rho g(h-x(t))$ the net hydrostatic pressure, and the normalization condition $\chi_{\mathrm{f}}+\chi_{\mathrm{H}}+\chi_{\mathrm{L}}=1$. Using Darcy's law, the fluid velocities are expressed as

$$
\begin{aligned}
v_{\mathrm{f}} & =\frac{k_{\mathrm{f}}}{\eta} \frac{\chi_{\mathrm{f}} \Delta p}{d_{\mathrm{f}}}, \\
v_{\mathrm{H}} & =\frac{k}{\eta} \frac{\chi_{\mathrm{H}} \Delta p}{d_{\mathrm{H}}}, \\
v_{\mathrm{L}} & =\frac{k}{\eta} \frac{\chi_{\mathrm{L}} \Delta p}{y-d_{\mathrm{H}}} .
\end{aligned}
$$

Due to mass conservation, $v_{\mathrm{L}}$ and $v_{\mathrm{H}}$ are not independent, see equation (5.1), and because the high-velocity cylinder has the same cross-section as the filter paper: $v_{\mathrm{f}}=$ $v_{\mathrm{H}}$. Under these conditions, and using equation (5.4), we find for $\chi_{\mathrm{f}}$

$$
\chi_{\mathrm{f}}=\frac{d_{\mathrm{eff}}}{d_{\mathrm{eff}}+d_{\mathrm{H}}\left(1+\left(y / d_{\mathrm{H}}-1\right) / \Lambda\right)},
$$

which shows that growth of the compactification layer in the low-velocity cylinder has a much smaller influence on the expulsion velocity of fluid than in the highvelocity cylinder since $\Lambda \approx 10$, see equation (5.9) for comparison. Given that $v_{\mathrm{f}}=\dot{x}$, using that $x(t)=\Delta \phi_{\mathrm{f}}\left(d_{\mathrm{H}}+\left(y(t)-d_{\mathrm{H}}\right) \Lambda\right)$, that $x_{\mathrm{H}} \equiv \Delta \phi_{\mathrm{f}} d_{\mathrm{H}}$ and equation (5.2), we find

$$
\dot{x}(t)=\frac{v_{\mathrm{i}}(1-x(t) / h)}{1+d_{\mathrm{H}}\left(1+\left(x(t) / x_{\mathrm{H}}-1\right) / \Lambda^{2}\right) / d_{\mathrm{eff}}},
$$

which reduces to equation (5.11) if there is no difference in cross-section between the low and high-velocity cylinders, i.e., if $\Lambda=1$. Moreover, given that $\Lambda \approx 10$ in the experiments, and because $d_{\mathrm{eff}}, h, d_{\mathrm{H}} \sim R_{\mathrm{in}}$, there is a small quantity, $\left(x / x_{\mathrm{H}}-1\right) / \Lambda^{2}=\left(y / d_{\mathrm{H}}-1\right) / \Lambda \ll 1$, and up to leading order we obtain

$$
\dot{x}=\frac{v_{\mathrm{i}}(1-x / h)}{1+d_{\mathrm{H}} / d_{\mathrm{eff}}},
$$

which shows that the hydrostatic pressure is predominantly dissipated in the filter paper and the compactified mayonnaise in the high-velocity cylinder, because they have a much larger fluid velocity than the low-velocity cylinder.

Equation (5.20) shows why after an initial decrease the expulsion rate becomes approximately constant, see Figure 5.2. The initial decrease is due to growth of the 
compactification layer in the high-velocity cylinder. As soon as it grows into the lowvelocity cylinder, however, the large value of $\Lambda$ causes the leading order contribution to the rate of fluid outflow to be approximately constant, provided $x(t) / h$ is small compared to unity. Therefore, the observed constancy of the rate of fluid expulsion is an artifact of the experimental setup.

Solving equation (5.19) with $x=x_{\mathrm{H}}$ at $t=t_{\mathrm{H}}$, we find

$$
\frac{x(t)}{h}=1+\alpha_{\mathrm{L}} W_{0}\left[-\frac{1-x_{\mathrm{H}} / h}{\alpha_{\mathrm{L}}} \exp \left(-\frac{1-x_{\mathrm{H}} / h+\left(t-t_{\mathrm{H}}\right) \Lambda^{2} / \tau}{\alpha_{\mathrm{L}}}\right)\right],
$$

where $\tau \equiv h \eta / k \rho g$ is the typical time for fluid outflow and

$$
\alpha_{\mathrm{L}} \equiv 1+\Lambda^{2} \frac{\Delta \phi_{\mathrm{f}} d_{\mathrm{eff}}}{h}+\frac{x_{\mathrm{H}}}{h}\left(\Lambda^{2}-1\right) .
$$

At $t=t_{\mathrm{L}}$ all mayonnaise in the low-velocity cylinder has compactified, i.e., $y=d_{\mathrm{H}}+h$ or $x=x_{\mathrm{L}}=\Delta \phi_{\mathrm{f}}\left[d_{\mathrm{H}}+h \Lambda\right]$. For $t>t_{\mathrm{L}}$ fluid can still be expelled, but the compactified network, consisting of random close packed oil droplets and possibly also starch components, has to compactify further. Therefore, the elasticity of the network becomes important and the problem becomes poroelastic, implying the poromechanical relaxation time to become important $\tau_{\mathrm{p}} \propto L^{2} \eta / k K$, with $L$, the total height of the mayonnaise in the jar, a measure for the distance over which fluid needs to be transported, and $K$ the bulk modulus of the random close-packed oil droplets. Also, the boundary conditions provided by the jar and the mayonnaise surface become important.

In this section we found the height of the column of expelled fluid as a function of time within the two-cylinders model. Given these results, the results for the height of the fluid column in the inner tube are fitted to the available experimental measurements in the next section.

\section{Comparison to experiment}

Here, we compare the theoretical results for the height of the column of expelled fluid in the inner tube, derived in the previous section, with the measurements, see Figure 5.2. The mass of expelled fluid $M_{\mathrm{ex}}$ can be calculated from $x(t)$ as $M_{\mathrm{ex}}(t)=\rho A_{\mathrm{in}} x(t)$, with $x(t)$ given by equation (5.12) and (5.21) in their respective 


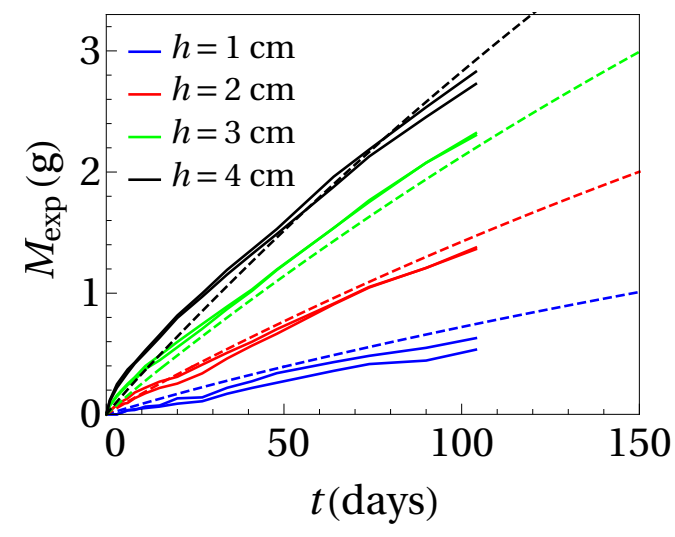

(A)

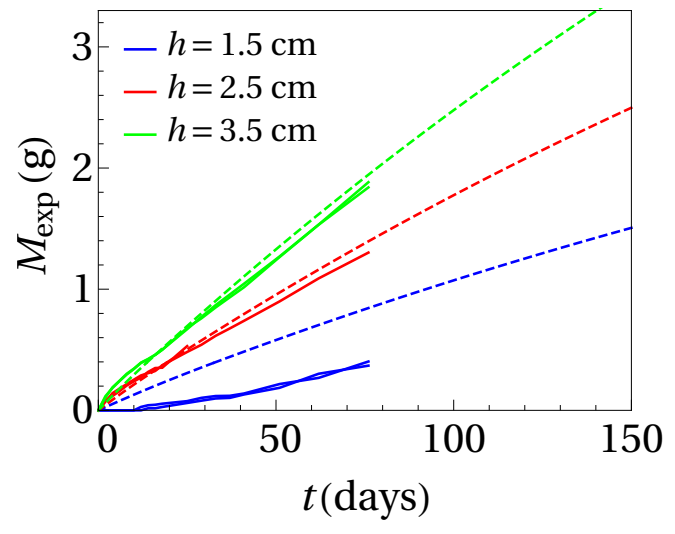

(B)

Figure 5.7: A) The measured amount of expelled fluid (full lines) for integer heights $h$ of the inner tube. The dashed lines give fits of the results of the clogging model, assuming the change in the volume fraction of the aqueous phase due to compactification to be $\Delta \phi_{\mathrm{f}}=$ 0.10 , the average oil droplet radius to be $R_{\mathrm{oil}}=1 \mu \mathrm{m}$, and by taking $d_{\mathrm{eff}}=9.0 \mathrm{~cm}$ and $d_{\mathrm{H}}=1.0 \mathrm{~cm}$ as fitted parameters. B) Full lines give the amount of expelled fluid for halfinteger heights of the inner tube. The dashed lines give the prediction of the two-cylinders model using the fitted parameters from $\mathbf{A}$.

time domains. We fix the permeability $k$ of the compactification layer by assuming Kozeny-Carman for the random close packed oil droplets, see equation (5.6), with an average oil droplet radius of $R_{\mathrm{oil}}=1 \mu \mathrm{m}$ and a volume fraction of the aqueous phase of $\phi_{\mathrm{aq}}=\phi_{\mathrm{aq}, 0}-\Delta \phi$, with $\phi_{\mathrm{aq}, 0}=0.48$ the uncompactified volume fraction of the aqueous phase and $\Delta \phi_{\mathrm{f}}=0.10$ the change in fluid volume fraction for random close packing of the oil droplets in the compactification layer to occur. Finally, we take the fluid viscosity as $\eta=10 \mathrm{mPa} \cdot \mathrm{s}$. We fit the model to the experiments with integer height of the inner tube by setting the effective length of the clogged filter paper as $d_{\text {eff }}=9.0 \mathrm{~cm}$ and the length of the high-velocity cylinder as $d_{\mathrm{H}}=1.0 \mathrm{~cm}$, see Figure 5.7A for the results. The result that $d_{\mathrm{H}}=1.0 \mathrm{~cm}$ suggests that the cross-section of the fluid flow grows significantly on the order of $1 \mathrm{~cm}$ away from the filter paper, in agreement with the results in section 5.2. Moreover, as the effective length is much larger than $d_{\mathrm{H}}$, most of the hydrostatic pressure is dissipated in the filter paper.

Given the definition of the effective length, see section 5.4, the total resistance against fluid flow is given by the sum of the effective length of the clogged filter paper $d_{\text {eff }}$ and the length of the high-velocity cylinder $d_{\mathrm{H}}$, because, as shown in section 5.4.2, 
to good approximation all hydrostatic pressure is dissipated in these parts of the experimental setup. Therefore, one can think of the experiments in the constant expulsion rate regime as a single layer of random close packed oil droplets of length $d_{\mathrm{eff}}+d_{\mathrm{H}}=10 \mathrm{~cm}$, in which all hydrostatic pressure $\rho g h$ is dissipated.

As the clogged filter paper sets the initial velocity of fluid outflow, see section 5.4, initially $100 \%$ of the hydrostatic pressure is dissipated in the filter paper. From equation (5.18), with $\left(y / d_{\mathrm{H}}-1\right) / \Lambda \ll 1$, we find that to leading order $90 \%$ of the hydrostatic pressure is dissipated in the filter paper in the constant expulsion regime, implying the clogged filter paper to be the dominant factor determining the outflow of fluid throughout the experiment.

Using the fitted values, we predict the expulsion for the experiments with half-integer values for the height of the inner tube, see Figure 5.7B. These predictions agrees reasonably well with the experiments, except for $h=1.5 \mathrm{~cm}$, as expected, because of the lag phase, see section 5.2. This delay might be caused by some slow timedependent process in the clogged filter paper, allowing fluid to pass only after 10 days.

To summarize, the measured expulsion of fluid from low fat mayonnaise can be fitted by assuming the filter paper to be clogged with oil droplets, and by using the results of the two-cylinders model, with reasonable values for the effective length of the filter paper $d_{\mathrm{eff}}$ and the length of the high-velocity cylinder $d_{\mathrm{H}}$. Therefore, we deem the hypothesis of a clogged filter paper and accompanying compactification layer to be a possible mechanism for fluid expulsion from model low fat mayonnaise. The additional experiments described in the next section, however, suggest the filter paper not to be clogged.

\subsection{Validation experiments}

In section 5.4, the measured expulsion in the syneresis experiments, see Figure 5.2, is explained by presuming the filter paper to be clogged with oil droplets. To test whether oil droplets indeed clog the filter paper in the experiments, several additional experiments were conducted.

As a reference experiment, the inner tube of the experimental setup is filled with 

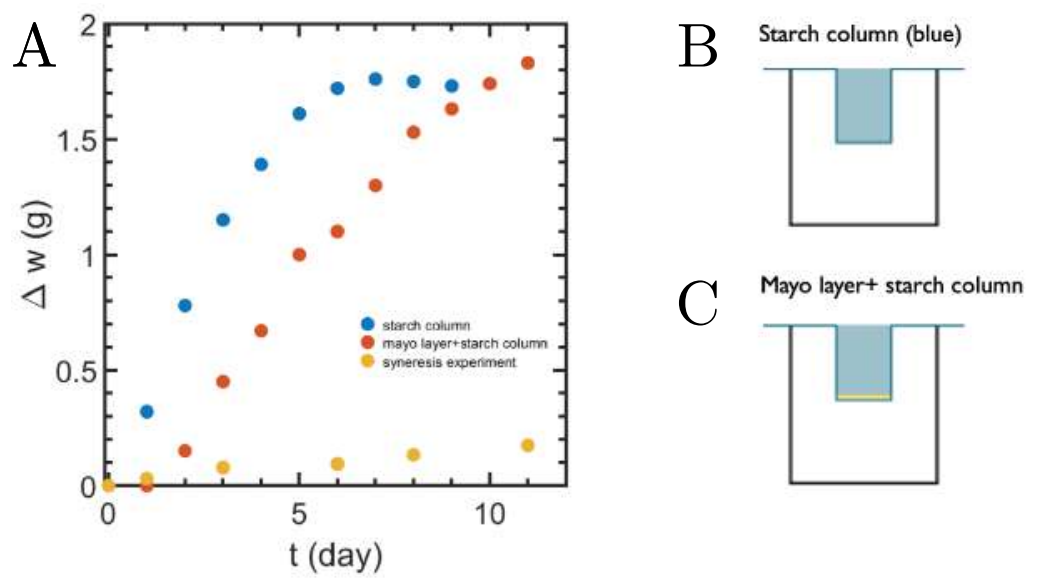

Figure 5.8: Schematic (B and $\mathbf{C})$ and results $(\mathbf{A})$ of the mayonnaise layer experiment to test whether clogging of the filter paper is significant. B) The inner tube, of height $h=2 \mathrm{~cm}$, is filled with starch paste as a reference experiment. C) The inner tube is filled with starch paste with a small layer of model low fat mayonnaise in between the paste and the filter paper. A) The weight of expelled fluid from the tube into the jar as a function of time $t$. The appurtenant syneresis experiment from Figure 5.2 (yellow dots) is given for comparison.

starch paste while leaving the jar empty, see Figure 5.8B, and the expulsion of fluid into the jar is measured. Next, in a so-called mayonnaise interlayer experiment we fill the inner tube with starch paste, but with a small, yet macroscopic, layer of mayonnaise in between the starch paste and the filter paper, see Figure 5.8C. We measure the mass of expelled fluid in the jar as a function of time, see Figure 5.8A. If oil droplets indeed clog the filter paper, the expulsion in the interlayer experiment is expected to be similar to the appurtenant syneresis experiment in Figure 5.2, where the inner tube has the same height $h=2 \mathrm{~cm}$ as the starch paste column, because in section 5.4 .2 it was found that at least $90 \%$ of the hydrostatic pressure is dissipated in the clogged filter paper. We observe, however, that the interlayer experiment exhibits similar fluid expulsion as the reference experiment, see Figure 5.8A. As both the reference and the interlayer experiment have an expulsion rate of about an order of magnitude larger than the appurtenant syneresis experiment, these results suggest the filter paper not to be clogged with oil droplets.

In the fluid expulsion experiments both the jar and the syneresis tube are filled with model low fat mayonnaise and left for approximately one week before initiation of the experiment. Next, the experiment is initiated by aspiration of the mayonnaise in the tube by a vacuum pump. In principle, this aspiration may cause clogging 

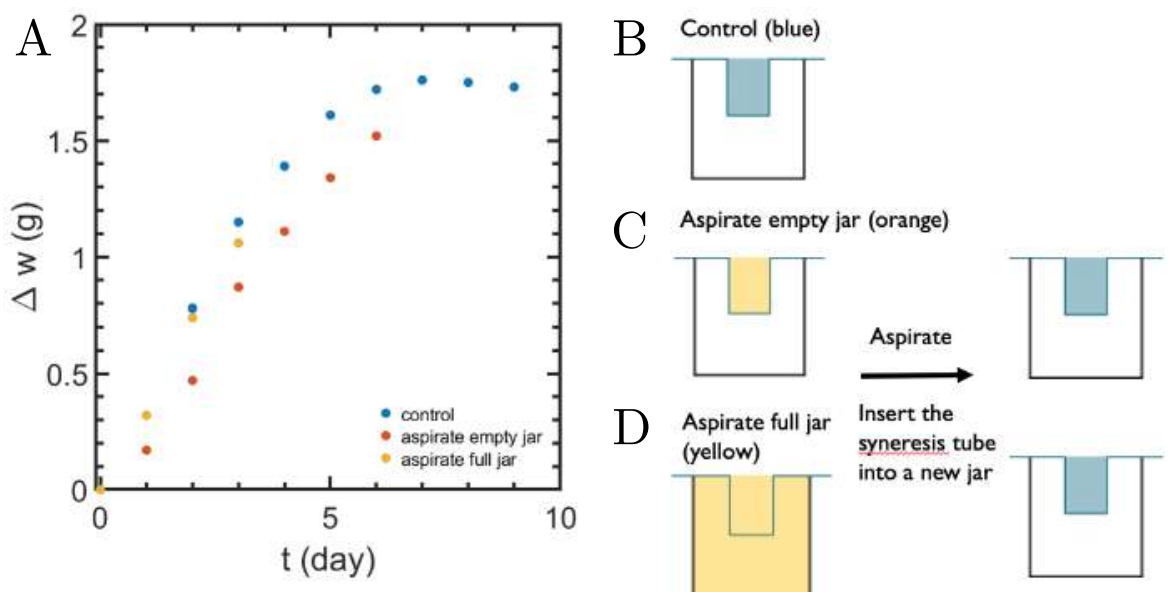

Figure 5.9: Schematics (B, C and $\mathbf{D})$ and results (A) of the experiments to test whether aspiration of model low fat mayonnaise causes clogging of the filter paper. B) In the control experiment the inner tube is filled with starch paste. C) The inner tube is first filled with model low fat mayonnaise and left for one week. Afterwards, the mayonnaise is aspirated and replaced by starch paste. D) Same as $\mathbf{C}$ but both the inner tube and the jar are filled with mayonnaise. A) The mass of expelled fluid from the inner tube into the jar as a function of time. No significant difference is observed, suggesting filter paper clogging to be irrelevant.

by sucking mayonnaise from beneath the filter paper through the filter paper. To test this we conduct the following experiments. First, a syneresis tube filled with mayonnaise is aspirated and the mayonnaise is replaced by starch paste, see Figure 5.9C. Also, a setup where both the jar and the tube are filled with mayonnaise is aspirated, after which the inner tube is filled with starch paste, see Figure 5.9D. The expulsion characteristics of these are compared to the control experiment in which the inner tube was filled with starch paste to start with, see Figure 5.9B. As the difference in expulsion rates between the three experiments is negligible, see Figure 5.9A, aspiration does not seem to clog the filter paper.

From the mayonnaise layer and aspiration experiments we conclude that clogging of the filter paper is insignificant for the expulsion characteristics we measure in Figure 5.2. Therefore, instead of the clogged filter paper hypothesis, we deem a double network in the mayonnaise to be responsible for the measured expulsion, see the next section. 


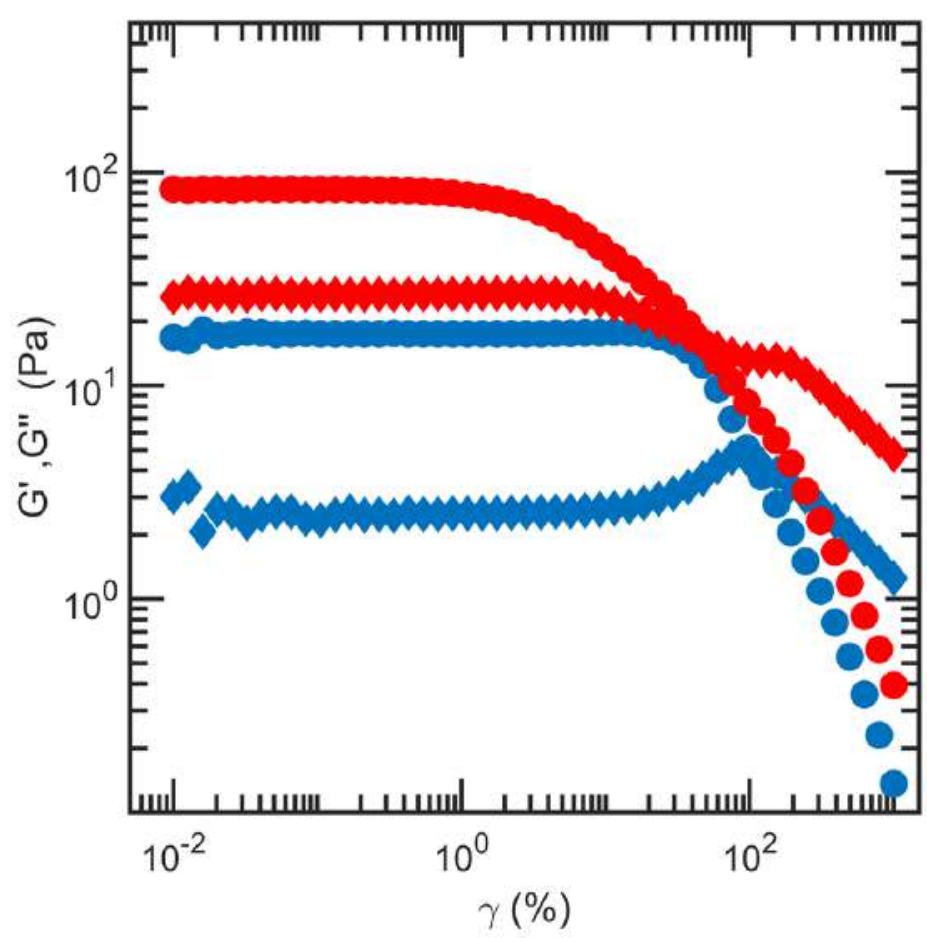

Figure 5.10: The storage $G^{\prime}(\bullet)$ and loss modulus $G^{\prime \prime}(\diamond)$ of the model low fat mayonnaise (red) and the starch paste (blue) as a function of shear strain. The peak in $G^{\prime \prime}$ for the starch paste is typical for a viscoelastic complex fluid that behaves as a solid at smalls strains but yields and flows at larger strains [209].

\subsection{Double network}

As shown in the previous section, clogging of the filter paper does not seem to determine the observed outflow of fluid. Another possible mechanism governing the outflow of fluid is the existence of a double network in the model low fat mayonnaise which yields and reforms during fluid outflow.

From the stress sweep experiment, see Figure 5.5, we find the yield stress of the starch paste and the model low fat mayonnaise to be approximately equal, implying solid-like properties for both materials. The aqueous phase, however, does not seem to have a yield stress, implying the extra ingredients in the aqueous phase, relative to the pure starch paste, to somehow destroy the starch network. To further test the rheo- 
logical properties of the model low fat mayonnaise and the starch paste, their storage and loss moduli have been measured, see Figure 5.10. The starch paste and the model low fat mayonnaise yield at, respectively, about $10 \%$ and $1 \%$ of shear strain, implying solid properties for both materials under small stresses. As the storage modulus of the model low fat mayonnaise before yielding is about $100 \mathrm{~Pa}$ and that of the starch paste about $20 \mathrm{~Pa}$, the presence of the oil droplets seems to enhance the solid properties. Indeed, an independent colloidal network could be formed through depletion interaction among the oil droplets caused by dissolved starch constituents, e.g., amylose [207, 210-212]. Moreover, the loss modulus of the starch paste shows a peak, typical of a viscoelastic complex fluid [209]. The loss modulus of the model low fat mayonnaise, on the other hand, seems to be a sum of a solid and a viscoelastic complex fluid because of the double plateau. This suggests the oil droplets somehow restore the viscoelastic properties of the starch in the aqueous phase. The starch and the oil droplets in the model low fat mayonnaise thus seem to form a double network.

As the hydrostatic pressure is in the order of $100 \mathrm{~Pa}$, we can not generally expect the whole double network to remain intact during the experiment. On the other hand, as verified in the previous section, the network does not yield in such a way that fluid is pushed through the filter paper while the filter paper becomes clogged with oil droplets. Therefore, we hypothesize both the oil droplet and the starch network to yield and reform by the generation of new bonds, the details of which depend on, for example, the dynamic viscosity of the continuous phase, the strength of depletion interactions, the size of the oil droplets and the connectivity of the oil droplet network [213-216]. Therefore, the double network could have a mechanical strength while at the same time being compressed, implying the process of fluid expulsion to be a poromechanical problem from the start of the experiment, contrary to the assumptions of the filter paper clogging hypothesis, see section 5.4.

Using the Terzaghi effective stress, we decompose the total stress in the model low fat mayonnaise as the sum of the Terzaghi effective stress, stemming from the mechanical contribution of the double network, and the fluid pressure [23, 27]. Because the yield stress is very small compared to the hydrostatic pressure, however, we neglect the Terzaghi effective stress. Also, as shown in section 5.4.2, the striction of the fluid flow through the mayonnaise causes nearly all hydrostatic pressure to be dissipated in a high fluid velocity region near the filter paper of size $d_{\mathrm{H}}$, independent of the tube 
height $h$ because the rate of dissipation is proportional to the fluid velocity according to Darcy's law. Therefore, assuming all hydrostatic pressure to be dissipated in the high velocity cylinder, $d_{\mathrm{H}}$ can be estimated using Darcy's law.

To use Darcy's law, the permeability of the low fat mayonnaise needs to be known. The double network is expected to have a permeability predominantly determined by the oil droplets, as the experiments in Figure 5.8 show that the initial rate of fluid expulsion for model low fat mayonnaise is about an order of magnitude smaller than that of the starch paste. By treating the double network as a porous bed of oil droplet particles, we estimate the total length of the bed $d_{\mathrm{H}}$ using the Kozeny-Carman expression for the permeability [27], which describes laminar fluid flow through a packed bed of oil droplets as a collection of curved tubes with diameter comparable to the oil droplets. In this way, the superficial velocity $v$ through the high-velocity cylinder and the hydrostatic pressure difference generated by the mayonnaise above the filter paper $\Delta P=-\rho g h$, can be related as

$$
v=-\frac{\left(\Phi R_{\mathrm{oil}}\right)^{2}}{45 \eta} \frac{\phi^{3}}{(1-\phi)^{2}} \frac{\Delta P}{d_{\mathrm{H}}},
$$

with $\phi$ the volume fraction of fluid, $\eta$ the dynamic viscosity of the expelled liquid, $\Phi$ the sphericity of the oil droplets, and $R_{\text {oil }}$ the average oil droplet radius. As we assume all of the low fat mayonnaise to be compressed from the start, we do not expect any appreciable change in the volume fraction of the aqueous phase during the experiments. Taking into account the $7 \mathrm{wt} \%$ of egg yolk in the aqueous phase, we estimate $\phi=\phi_{\mathrm{aq}}-\phi_{\text {egg }}$, where $\phi_{\mathrm{aq}}=0.48$ is the initial volume fraction of the aqueous phase and $\phi_{\text {egg }}=0.07$ is estimated to be equal to its mass fraction, giving $\phi \approx 0.41$. The order of magnitude of the average size of the oil droplets can be estimated from the SEM image of the model low fat mayonnaise, see Figure 5.1, giving $R_{\mathrm{oil}}=1 \mu \mathrm{m}$. Also, the SEM image suggests the particles to be approximately spherical, implying $\Phi \approx 1$. Based on the average expulsion rate $\bar{r}$, see Figure 5.3, we estimate $v / h \approx 2.7 \cdot 10^{-8} \mathrm{~s}^{-1}$, where we used $\bar{r}=\rho A_{\text {in }} v$, with $A_{\text {in }}$ the end area of the inner tube. Finally, we estimate the dynamic viscosity of the expelled fluid to be about ten times the viscosity of water, i.e., $\eta=10 \mathrm{mPa} \cdot \mathrm{s}$. Using these estimated values, we find $d_{\mathrm{H}}=16 \mathrm{~cm}$, which differs from the dimensions of the experimental setup by one order of magnitude. In fact, in section 5.2 and 5.3 it was argued that $d_{\mathrm{H}}$ should be about $1 \mathrm{~cm}$. This discrepancy could be explained in the following manner. 
A starch paste can retrograde into a firm gel after it has been made [217]. Suppose that in the low fat mayonnaise starch retrogradation occurs, possibly in interplay with the oil droplets. This gel formation need not be homogeneous throughout the model low fat mayonnaise. Therefore, at the initiation of a syneresis experiment only parts of the double network could be prone to yielding and subsequent reformation. Then, the fluid velocity through the yielded mayonnaise would actually be higher than the estimated $v$, giving rise to a smaller value of $d_{\mathrm{H}}$. Moreover, as starch retrogradation initially involves the relatively fast recrystallization of amylose molecules, followed by the slow crystallization of amylose pectin molecules [217], the decrease in expulsion rate after initiation of the experiment could be caused by ongoing starch retrogradation. Finally, the lag phase in the $h=1.5 \mathrm{~cm}$ experiments could be caused by an energy barrier to yielding of the double network which builds up in the process of starch retrogradation, only to be overcome after the lag phase.

\subsection{Conclusions and discussion}

The mechanism governing syneresis in low fat mayonnaise has been investigated by conducting syneresis experiments on a model low fat mayonnaise. In these experiments a height difference, simulating a scoop taken from the mayonnaise, is established. The expulsion rate of fluid is found to be proportional to the induced height difference. In first instance, the rate of expulsion is assumed to be set by the filter paper in the experimental setup. Taking into account the geometry of the setup in a two-cylinders model, the clogged filter paper can explain the measured fluid expulsion. Further experiments, show, however, that the filter paper is not clogged with oil droplets. Next, measurements of the storage and loss modulus of both the model low fat mayonnaise and the starch paste where the first is based on, suggest a network of starch components and a network of oil droplets to be present in the low fat mayonnaise, where the latter forms through depletion interaction mediated by dissolved starch components. This network is supposed to yield and reform during fluid expulsion, thereby forming a porous medium for fluid to flow through. Again, simplifying the fluid flow as a one-dimensional flow through two cylinders, we estimate the size of the high-velocity cylinder to be of the order of $10 \mathrm{~cm}$. Measurements of the average rate of fluid expulsion suggest the size of the high-velocity cylinder to be of the order of $1 \mathrm{~cm}$, however. This discrepancy could be caused by starch retrogradation 
in the low fat mayonnaise, which causes only parts of the double network to yield, therefore producing a higher fluid velocity through the yielded mayonnaise and a lower estimation of the size of the high-velocity cylinder. 


\section{Chapter 6}

\section{General discussion}


In this thesis we have investigated a relatively large variety of experimental systems, ranging from synthetic- to fibrin fiber hydrogels. Our investigations were largely connected by focusing on the characterization of the poromechanical properties of gels. Below, we discuss our findings and provide some directions for future research.

\subsection{Hydrogel dynamics in polymer solutions}

In the first chapter, we focused on the swelling and compression dynamics of hydrogels in (concentrated) polymer solutions. The hydrogels were observed to exhibit non-monotonic swelling and deswelling, an observation which asked for explanation. Through a numerically solved relaxational dynamics model, we could give an accurate account of the measured volume of a hydrogel as a function of time. Through this account, we were able to estimate the bulk modulus and permeability of the hydrogel network, as well as the diffusion constant of the dissolved polymer molecules and the solvent quality change they bring about for the hydrogel network. On the other hand, through a poromechanical approach, we theoretically examined the response of a hydrogel on the diffusing polymers from a diluted solution, and we were able to formulate an explicit expression for the displacement field of the hydrogel network and the concentration profile of polymer molecules in the hydrogel. Assuming the dominant contribution to the hydrogel dynamics of a diluted solution to also be the dominant response of a hydrogel in a concentrated solution, we constructed a closed-form model for hydrogel dynamics in concentrated polymer solutions. Using this model, one can extract the bulk modulus, the permeability, the diffusion constant and the hydrogel-polymer interaction coefficient from volume measurements on a hydrogel.

Conventionally, the permeability of hydrogels is established through a flow assay [146], a complicated measurement compared to the mass measurements on hydrogels we considered. The bulk modulus of hydrogels can be measured by Capillary Micromechanics [122] and the diffusion coefficient of polymer molecules in hydrogels can be determined by, among others, nuclear magnetic resonance techniques [138, 218]. These three material parameters are, in principle, readily accessible through the dominant-mode model combined with hydrogel volume measurements. We expect the dominant-mode model to be especially useful as a novel method to charac- 
terize the diffusion coefficient of guest molecules in (soft) hydrogels at different $\mathrm{pH}$ and temperatures. This method distinguishes itself from existing techniques [138] by using relatively simple mass measurements, which might even develop into a highthroughput technique with appropriate automation. Limitations of the method are that the diffusion time scale needs to be (much) larger than the (de)swelling timescale of the hydrogel, such that diffusion-induced (de)swelling can be clearly observed, as well as that the magnitude of (de)swelling needs to be measurable. A systematic assessment of the accuracy of the model would first be necessary, however, which is a task for future research. In practice, we experienced problems with convergence of fitting the data, which forced us to fix a material parameter from conventional measurement techniques in some of the experiments we analyzed. Part of the convergence problems might be explained by the fact that the PEO polymer solutions were polydisperse. Structurally, the convergence problems might be solved by making mass measurements on the hydrogel at equal time intervals. In this manner the longtime processes of slow (de)swelling effectively gain more weight in the fit routine. Due to the large time scales involved, however, many mass measurements would be required, the automation of which could be an object for future research.

Both the relaxational dynamics and the dominant-mode model show that nonmonotonic (de)swelling of hydrogels arises from an interplay between the osmotic pressure of the osmolyte molecules and the change in solvent quality for the hydrogel network that these molecules may bring about. When the hydrogel is immersed in polymer solution, it is compressed by the osmotic pressure difference between the inside and the outside of the hydrogel. Upon diffusion of osmolyte molecules into the hydrogel, however, this pressure difference decreases while the solvent quality can change. The combination of these two processes determines whether the hydrogel swells or compresses due to the diffusion of polymer molecules. As these two different processes produce a single measurable characteristic, one may expect that there are some caveats when deducing the material properties from a volume measurement. The 'mapping' of the measured volume to the material properties through the dominant-mode model may be, so to say, non-surjective. When an initially swollen hydrogel exhibits an undershoot in the measured volume, however, the measurement has four clear characteristics on the basis of which the bulk modulus and permeability of the hydrogel network, and the diffusion constant and solvent quality change of the polymer molecules, can be characterized, possibly uniquely. Initially, the hydrogel compresses fast, while for later times it slowly reswells. The magnitude and the time 
scale of the former correspond to, respectively, the bulk modulus and permeability of the hydrogel network. The magnitude and time time scale of the latter is caused by, respectively, the change in solvent quality brought about by the polymer molecules and their diffusion constant. Our results suggest that for a non-monotonic evolution of the hydrogel volume our model provides a 'bijective' mapping. Whether this holds in a robust manner, however, is a subject for future research. If a compressed hydrogel does not exhibit an undershoot, but its volume decreases monotonically, the distinction between the fast initial and the slow asymptotic compression is less clear and may lead to a non-unique determination of the material properties. In this case, a swelling experiment on an initially dry hydrogel in the polymer solution may be more appropriate, for it can exhibit an overshoot.

\subsection{Compression of (bio)polymer gels}

As we have seen in the introduction, hydrogels are often used in biomedical applications. Considering the mechanical aspects of living creatures, one encounters biopolymer networks and tissues which exhibit gel dynamics due to their porosity and elasticity. We investigated a novel method to determine the poromechanical properties of (bio)polymer gels and tissues from a ramp compression test in a commercial rheometer. As biopolymer gels, e.g., fibrin gels, are prone to stick to the rheometer plates during compression, we developed a novel approximate solution to the poroelastic equations of motion: a closed-form expression for the displacement field of the gel network and the flow field of the fluid. With this solution, the measured force in a ramp compression test can be interpreted to yield the permeability and (effective) elastic properties of the gel network. Furthermore, we found our approximate solution, with appropriate phenomenological extensions, to be capable of probing the poromechanical properties of fibrin gels in the nonlinear regimes of large compression and strain stiffening. In particular, the contribution to the normal force in the linear regime was found to hold at large compression with a strain-dependent permeability, and we could model strain stiffening during compression with a stepwise increase in the shear modulus of the gel network. Often, research focuses on the discovery of materials with low surface friction [219]. Methodological reasons for this are that with zero surface friction the poroelastic equations can be solved exactly for plate-plate compression [176] or be well-approximated in indentation testing [220, 
221]. In practice, however, small surface friction coefficients can already make a large impact on the measured normal force in a compression experiment [222]. Our approach avoids the need to decrease the friction coefficient of a hydrogel through, e.g., lubrication, or to limit oneself to low-friction materials, but fully incorporates the friction between the hydrogel and the rheometer plates. In case the gel might (partially) slip over the rheometer plates, it may be bonded by using, e.g., surgical glue [222].

Our approximate solution of the compression of a bonded disk-like gel is essentially different from that of a frictionless gel, for in a bonded gel the network is heavily strained by bulging of the network. The most important approximation we have made, is the neglect of the influence of the free boundary conditions. In very slow compression relative to the pressurizing time, that is, the time in which the fluid pressure becomes maximal, the (radial) relaxation stemming from the free boundary conditions can occur during the compression process. Indeed, in the slow-compression experiment displayed in Figure 3.11, the normal force might to increase sub-linearly due to radial relaxations, though this is difficult to judge because of the measurement uncertainty. If the compression time is of the same order and larger than the pressurizing time, however, we seem to observe a linear increase in the normal force, see Figure 3.1, which suggests that our approximate model is indeed applicable. A systematic investigation of the influence of the free boundary conditions by a numerical solution of the equations of motion through, e.g., finite element analysis, is a subject for future research.

A remarkable finding from our analysis of the compression experiments on largepore fibrin gels is that the (effective) elastic properties of the fibrin network during compression are found to depend on the strain rate. This suggests that the elasticity of the fibrin fibers depends on the pressure of the fluid, which scales proportionally to the strain rate. It is known that protein structure depends on the pressure of the surrounding fluid [223], suggesting that the fibrinogen protein structure might change with fluid pressure, thereby giving rise to strain rate dependent elasticity of the fibrin network in a compression experiment. Moreover, in the compression experiments on small-pore fibrin gels, we found the fibrin network to strain stiffen during compression. We modelled this by assuming a single stepwise increase in the shear modulus of the fibrin network at the early stage of fluid pressure build-up in the gel. After stiffening, the fluid pressure increases to its maximal value which implies, accord- 
ing to the approximate model, that the fibrin network is increasingly strained also after stiffening. From earlier work, however, stiffening is found to increase continuously with strain on the fibrin network [100], which suggests that the fibrin network increasingly stiffens throughout compression. Therefore, the increase of the fluid pressure seems to inhibit strain stiffening. Possibly, this inhibition is also related to a change in the structure of the fibrinogen monomers with fluid pressure. The influence of fluid pressure on the elastic properties of fibrin networks is a subject for future research.

In the large compression experiments on fibrin gels, we only considered the measured normal force when the fluid pressure was (presumed to be) maximal. Therefore, we did not investigate whether the exponential relaxation in our approximate solution is also applicable when the fluid pressure builds up during the compression steps at large compressive strain. Possibly, our approximate solution can account for the full timevarying normal force at large compressive strain if the dependence of the pressurizing time on the compressive strain is taken into account through the permeability; this provides a question for future research.

Using a cubic lattice model for the fibrin network, we found from our analysis of compression experiments on small-pore fibrin gels that the mass density per unit of fiber length decreases with increasing concentration of the initial fibrinogen solution, or equivalently, the number of protofibrils per fibrin fiber cross-section decreases with fibrinogen concentration. An earlier work has suggested that a higher supply rate of fibrin monomers during polymerization, which can be expected to increase with fibrinogen concentration, gives rise to more branching of fibrin fibers and shorter fiber segments between branch points [224]. Possibly, branching inhibits the lateral aggregation of protofibrils, thereby lowering the number of protofibrils per fibrin fiber with increasing initial fibrinogen concentration.

We hope that our approximate model will stimulate further research into the mechanisms, structural motifs and driving forces of lateral protofibril aggregation, as these are mostly still unknown [94]. For example, if one wishes to investigate the influence of, say, molecule A on lateral protofibril aggregation, one can proceed in the following manner. The dependence of the permeability $k\left(c_{\mathrm{A}}\right)$ on the concentration of the molecule $c_{\mathrm{A}}$ can be inferred from compression experiments using our approximate model. Assuming the ratio of the protofibril mass to the protofibril length to be independent of the concentration of molecule A, the cubic lattice model asserts that the 
permeability is proportional to the number of protofibrils per fibrin fiber cross-section $N_{\text {cross }}\left(c_{\mathrm{A}}\right)$, that is, $k\left(c_{\mathrm{A}}\right) \propto N_{\text {cross }}\left(c_{\mathrm{A}}\right)$, thereby relating the measured permeability to the number of protofibrils per fibrin fiber cross-section.

\subsection{Plate-sphere compression}

In compression tests on solids, one may have problems with misalignment of the rheometer plates, which can cause regions of strong plastic deformation in elasticplastic materials in an uncontrolled manner. Moreover, some materials are shapelimited, or highly prone to buckle, such that they cannot be given a rod-like shape which minimizes the effects of friction in the determination of the Young's modulus in a compression test. In the latter case, the materials need to be lubricated, but lubrication may render the positioning of a compression sample problematic. Therefore, we investigated a compression test with a spherically tipped plate. This geometry would make the test less sensitive to plate misalignment, for the rotation of a sphere produces another spherical tip. Moreover, a spherically tipped plate would facilitate the positioning of the sample. But how does a compressed solid respond to compression with a spherically tipped plate? To answer this question, we solved the force balance of a linear elastic solid compressed with a parabolically curved plate using a novel perturbation approach which allowed to decompose the problem into many, infinitely many, subproblems, yielding a solution which provides the exact magnitude of the required load for compression of a sample, free of surface friction, having any aspect ratio. In the limit of a disk-like sample, this solution becomes exact, and in the limit of a rod-like sample, it pertains that the normal force is equivalent to a plate-plate compression displacing an equal quantity of volume.

\subsection{Scoop syneresis}

We investigated a problem with industrial relevance: the expulsion of fluid from low fat mayonnaise. To attack this problem, we formulated a two-cylinders model to take into account the geometry of the experimental setup. With this model, we first investigated the appropriateness of the setup, that is, we considered whether clogging of the membrane in the setup could explain the observed expulsion of fluid. Through 
experiments, it was found that the membrane was not clogged, however. Furthermore, given that low fat mayonnaise is a complex material, we probed what the physicochemical origin of the observed expulsion process could be.

The model low fat mayonnaise which we have put under scrutiny was a far cry from consumer mayonnaise, for its yield stress $\sigma_{\mathrm{Y}}$ is in the order of tens of Pascal. This implies that the order of magnitude of the surface height inhomogeneities $h$ of the model low fat mayonnaise in a jar are expected to be no more than tens of micrometers $\left(\sigma_{\mathrm{Y}}=\rho g h\right)$, i.e., the mayonnaise has a practically flat surface. A more realistic model low fat mayonnaise with higher yield stress might give highly different expulsion characteristics, for the recipe of this mayonnaise can have very different concentrations of the ingredients, which may interact in unexpected ways. A rational design of low fat mayonnaise seems yet elusive. 



\section{Appendix A}

\section{Dimensonal analysis}

As an appendix to this thesis we introduce a part of physical analysis which is generally not very well known: dimensional analysis. It is applied within engineering [225] and applied physics [226, 227], and it is related to the dimensionless numbers arising within fluid dynamics, e.g., the Reynolds number Re. Dimensional analysis is very helpful to obtain a 'taste' of a physical problem before entering into a firstprinciple analysis: it can give an idea of the relevant relations between the physical characteristics pertaining to the system under consideration, and in some cases it may provide the solution.

In the following sections we briefly describe the main idea of dimensional analysis through some instructive examples, and we state its most important result: the $\Pi-$ theorem.

\section{A.1 Complete physical relations}

Dimensional analysis is founded on the elegant principle that any complete physical relation should hold irrespective of the fundamental units in which the physical quantities entering the relation are expressed [226]. As a simple example of a complete physical relation, one can consider the period $T$ for small oscillations of a rigid pendulum in the earth gravitational field when friction is negligible. Within classical mechanics, this period can be calculated in terms of the length of the pendulum $l$ 
and the gravitational acceleration $g$ as $T=2 \pi \sqrt{l / g}$. Irrespective of the fundamental units of length and time in which $T, g$, and $l$ are measured, the relation holds in the same form, therefore it is called complete.

Valid physical relations do not need to be complete. Suppose we would not know of classical mechanics and through experiment we find for the pendulum that $T=$ $C \sqrt{l}$, with $C$ a constant whose numerical value is equal to 2.01 when $T$ and $l$ are measured in, respectively, seconds and meters. The numerical value of $C$ in our experimental result will change if we measure the length in centimeters. In the result from classical mechanics quoted above, on the other hand, the value of the constant $2 \pi$ is unchanged by switching from one system of units to another, thereby making it a complete physical relation. The unit-invariance of complete physical relations is at the core of dimensional analysis.

At this point one may object that in the result of classical mechanics the gravitational acceleration $g$ acts effectively like the constant $C$ in the experimental relation when changing the units, that is, one could consider $C$ as a dimensional constant expressed as $C=2 \pi / \sqrt{g}$ : each incomplete physical relation can be made complete by introducing a dimensional constant.

The power of dimensional analysis is in situations in which the relevant physical constants are known beforehand, in other words, when the physical constants entering the equations of motion underlying the phenomenon are known. In the case of the period of a pendulum, for example, one does not consider the Boltzmann constant, as the problem is clearly in the realm of classical mechanics and not in that of thermodynamics. To know what the relevant constants are, however, one needs experience and theoretical formation.

\section{A.2 A frictionless pendulum}

To make use of the unit-invariance of complete physical relations, one can consider a physical relation between some quantities which is of unknown form, and presuppose that it is complete. That is, one assumes to have complete knowledge of the relevant physical quantities in the physical relation. Returning to the example of the pendulum, suppose the period of oscillation is not known explicitly through classical mechanics. One can write a general functional relationship between the period of 
oscillation $T$ and other physical quantities which supposedly determine the period. For example, one could write

$$
T=f_{1}(g, l, \theta),
$$

where $f_{1}$ is some unknown function and $\theta$ is the dimensionless initial angle in radians under which the pendulum is released, not necessarily small. One could argue for the relevance of the quantities in this relation by asserting that the pendulum makes a falling movement in the earths gravitational field, implying the gravitational acceleration $g$ to be into play. Moreover, the larger the length of the pendulum is, the larger is the distance of the tip to travel in one period, pointing towards a dependence on $l$. Finally, for a pendulum of a given length $l$ the initial angle $\theta$ of the pendulum could be relevant as it determines which part of a circle the pendulum runs through in one period, therefore one expects a larger period for a larger initial angle $\theta$. On the other hand, to neglect quantities like the mass of the pendulum and the friction constant of the pendulum with the surrounding air is nontrivial. The latter rests on the presumption that friction is negligible, and the former presupposes theoretical formation that in free fall the mass of an object does not enter in the kinematic description.

Assuming equation (A.1) to be complete, the right hand side needs to be expressed in units of time, since the left hand side has units of time. Therefore, it has to be possible to form a quantity with units of time on the right hand side, and we can write in general

$$
T=\sqrt{\frac{l}{g}} f_{2}(g, l, \theta),
$$

where $f_{2}$ is another unknown function. Upon an arbitrary change of the unit of time in equation (A.2), it is necessary for the value of $f_{2}$ not to change, otherwise the relation between $T$ and $g, l$ and $\theta$ changes when changing the units, thereby rendering equation (A.2) incomplete. The numerical value of $g$, one of the arguments of $f_{2}$, does change by changing the unit of time, however, which implies that $f_{2}$ has to be independent of $g$. Next, by the same argument, we find $f_{2}$ to be independent of $l$. Equation (A.2) then becomes

$$
T=\sqrt{\frac{l}{g}} f_{2}(\theta),
$$

where $f_{2}(\theta)$ is a function to be determined through experiment or through classical mechanics. In fact, within classical mechanics $f_{2}(\theta)$ can be calculated to be $f_{2}(\theta)=$ 
$4 F(\pi / 2, \sin (\theta / 2))$, with $F$ the incomplete elliptic integral of the first kind. The above example shows that through the right assertion of the relevant quantities in a physical relation, that is, by writing down a complete physical relation, important results can be deduced on the basis of dimensional arguments alone. In this way, the experimental work of determining $f_{1}(g, l, \theta)$ in equation (A.1) is reduced by, say, two orders of magnitude in equation (A.3), where a function of only one argument needs to be determined.

\section{A.3 The П-theorem}

The supposed complete relation for a pendulum's period, equation (A.1), was a functional relationship between four quantities. After using dimensional arguments, however, it can be rewritten in terms of two dimensionless quantities, $T \sqrt{g / l}$ and $\theta$, in equation (A.3), thereby reducing the number of independent quantities by two. From dimensional analysis, it turns out that in general, when writing the physical relationship in terms of dimensionless groups, the number of independent quantities in a complete physical relation can be reduced by the number of fundamental units they are expressed in. In the above example, the fundamental units in which equation (A.1) was expressed were that of length and time, as the period could be expressed in seconds, the length in meters, and the gravitational acceleration in meters per second squared. Therefore, equation (A.1) was expressed in $4-2=2$ quantities in equation (A.3).

The $\Pi$-theorem can be stated as follows [226]. A complete physical relationship between $n$ quantities $a_{1}, a_{2}, \ldots, a_{n}$, given in general by

$$
f\left(a_{1}, a_{2}, \ldots, a_{n}\right)=0
$$

can be written as

$$
F\left(\Pi_{1}, \Pi_{2}, \ldots, \Pi_{n-m}\right)=0,
$$

which depends on $n-m$ dimensionless groups $\Pi_{1}, \Pi_{2}, \ldots, \Pi_{n-m}$ where $m$ is the number of fundamental units in which the $n$ dimensional quantities are expressed. These dimensionless groups are products of the original quantities where the each of the original quantities is raised to some power. 


\section{A.4 A pendulum with friction}

For the remainder of this section, it is instructive to illustrate the influence of presumptions on the results of dimensional analysis by returning to the example of the pendulum. If one would not have presupposed that friction is negligible, one should have taken the mass of the pendulum $m$ and the frictional constant of the pendulum with the surrounding air $\xi$ into consideration. In the laminar flow limit, $\xi \boldsymbol{v}$ is the frictional force acting on the center of mass of the pendulum for a center of mass velocity $\boldsymbol{v}$. Also, because friction destroys time translation symmetry, the time $t$ after release of the pendulum should be taken into account. Equation (A.1) would then have had seven relevant quantities, and by expressing the unknown function in terms of dimensionless groups, similar as in section A.2, equation (A.3) becomes

$$
T=\sqrt{\frac{l}{g}} f_{3}\left(\theta, \frac{\xi \sqrt{g l}}{m g}, \frac{t}{\sqrt{l / g}}\right)
$$

where we redefined $T$ as the time between two subsequent maximum extensions of the pendulum at, say, the positive angle side. As the original quantities are now expressed in three fundamental units: mass, length and time, the resulting number of dimensionless groups is $7-3=4$. We find a dimensionless group $\xi \sqrt{g l} / m g$ giving the ratio of the typical size of the frictional force to the gravitational force acting on the pendulum, therefore measuring the importance of friction relative to the driving force of the movement. Also, we find $t / \sqrt{l / g}$ as a measure of the number of periods which have been elapsed after release of the pendulum, in case of the underdamped limit.

The product of the two new dimensionless groups in equation (A.6), $(\xi \sqrt{g l} / m g)(t / \sqrt{l / g})=t \xi / m$, gives another dimensionless group determining the influence of friction on the behaviour of the pendulum. This group is the ratio of the typical quantity of momentum lost to friction after time $t, \xi \sqrt{g l} t$, to the typical quantity of momentum contained originally in a (hypothetical) free fall of the pendulum, $m \sqrt{g l}$. Because $f_{3}$ in equation (A.6) is a general relation, it can be reformulated as

$$
T=\sqrt{\frac{l}{g}} f_{4}\left(\theta, \frac{\xi \sqrt{g l}}{m g}, \frac{t \xi}{m}\right),
$$

Equation (A.7) shows that neglect of the influence of friction in this problem is the 
same as asserting that $t \xi / m \ll 1$, meaning that no significant quantity of the initial momentum has been lost to friction. The question of whether this 'practically frictionless' regime comprises a fraction of a period or several periods is subsequently given by $\xi \sqrt{g l} / m g$, the ratio of the typical size of the frictional force to the typical size of the gravitational force. When this group is also much smaller than one, it can be assumed that there is a number of periods in which the frictional force can be safely neglected.

The extension of the pendulum example to the frictional case shows that dimensional analysis can give quite precise information on the conditions for limiting cases in a physical problem. On the other hand, it should be noted that this example is well known from classical mechanics and simulations. For a problem where the determination of a complete set of quantities may be done, but for which the explicit equations of motion are either unknown or insolvable, the neglect of appropriate groups in a given limit is not necessarily straightforward. 


\section{Samenvatting}

Hydrogels bestaan in het algemeen uit een combinatie van een netwerk van vaste stof en een vloeistof die in dit netwerk doordringt. Interacties tussen de vaste stof en de vloeistof bepalen de respons van een hydrogel wanneer deze wordt verstoord door bijvoorbeeld externe mechanische of osmotische druk. De resulterende tijdsafhankelijke dynamiek van (ont)zwelling wordt gekarakteriseerd door de poromechanische eigenschappen van een hydrogel: de (visco)elasticiteit, plasticiteit en permeabiliteit. In dit proefschrift richten we ons voornamelijk op de vaststelling van de permeabiliteit en elasticiteit van hydrogels door middel van de modellering van de dynamica van enkele (ont)zwellingsprocessen bij synthetische hydrogels en biopolymeergels. Ook richten we ons op het meer algemene probleem van compressietesten op vaste stoffen waarbij we een nieuw soort geometrie van de testmachine behandelen en we maken een poromechanische studie van een industrieel relevant fenomeen: de spontane expulsie van vloeistof uit vetarme mayonaise.

In Hoofdstuk 2 behandelen we de zwelling en compressie dynamica van synthetische hydrogels in (geconcentreerde) polymeeroplossingen. We observeren niet-monotone (ont)zwelling bij de hydrogels, een observatie die om uitleg vraagt. Door middel van een numeriek model, gebaseerd op fenomenologische relaxionele dynamica, geven we een accurate beschrijving van het gemeten hydrogel volume als functie van de tijd. Met deze beschrijving verkrijgen we zowel schattingen van de compressiemodulus en de permeabiliteit van het hydrogel netwerk als van de diffusieconstante van de opgeloste polymeren in het hydrogel netwerk en de verandering in oplosmiddelkwaliteit die de polymeren teweeg brengen voor het netwerk. Verder onderzoeken we door middel van een poromechanisch model de theoretische respons van een hydrogel op een verdunde oplossing van polymeren en we formuleren een expliciete uitdrukking voor zowel het verplaatsingsveld van het hydrogel netwerk als voor het concentratie- 
profiel van polymeermoleculen in de hydrogel. Op basis van de aanname dat de dominante contributie aan de hydrogeldynamica voor een verdunde polymeeroplossing van eenzelfde vorm is als die voor een geconcentreerde oplossing, construeren we een gesloten-vorm model voor de dynamica van hydrogelen in geconcentreerde polymeeroplossingen. Gebruikmakend van dit model kan men de compressiemodulus, permeabiliteit, diffusieconstante en hydrogel-polymeer interactiecoefficient afleiden vanuit volumemetingen aan een hydrogel.

In Hoofdstuk 3 bestuderen we een nieuwe methode om de poromechanische eigenschappen van biopolymeergels en weefsels te karakeriseren door middel van een constante-snelheid compressietest in een commerciële rheometer. Aangezien biopolymeergels, bijvoorbeeld fibrinegels, zich sterk hechten aan de rheometerplaten gedurende compressie, ontwikkelen we een nieuwe benaderende oplossing van de poroelastische bewegingsvergelijkingen: een gesloten-vorm expressie voor het verplaatsingsveld van het gel netwerk en het stromingsveld van de vloeistof, waarbij de gel gebonden is aan de rheometerplaten. Gebruikmakend van deze oplossing kan men de gemeten normaalkracht in een constante-snelheid compressietest interpreteren om zo de permeabiliteit en de (effectieve) elastische eigenschappen van het gel netwerk te verkrijgen. Bovendien kan men met onze benaderende oplossing, gegeven de nodige fenomenologische extensies, de poromechanische eigenschappen van fibrinegels in het niet-lineaire regime van grote compressie en rekverstijving bestuderen. Meer precies, we vinden dat de vorm van de normaalkracht in het lineaire regime behouden blijft tijdens grote compressie mits een rek-afhankelijke permeabiliteit wordt gebruikt en de rekverstijving tijdens compressie wordt gemodelleerd met een stapsgewijze toename in de schuifmodulus van het gel netwerk.

In Hoofdstuk 4 behandelen we compressietesten op vaste stoffen. We bekijken een nieuw soort geometrie van testmachines om zo de elasticiteitsmodulus van vormbeperkte materialen te bepalen alsmede die van elasto-plastische materialen die sensitief kunnen zijn voor foutieve uitlijning van vlakke compressieplaten. In deze nieuwe compressie geometrie, waarbij de bovenste plaat sferisch is gebogen, rapporteren we de kracht-rek relatie van een lineair elastisch materiaal door middel van een perturbatieve oplossingstrategie toegepast op de Navier-Cauchy vergelijkingen.

Tenslotte bestuderen we in Hoofdstuk 5 de spontane expulsie van vloeistof, dat wil zeggen de synerese, van een industrieel relevant systeem: een modelsubstantie voor vetarme mayonaise. Door de mayonaise als een poreus materiaal te beschouwen, en 
door de geometrie van de experimentele opstelling met een twee-cylinder model in ogenschouw te nemen, beschrijven we de gemeten expulsie van vloeistof en maken een kritische evaluatie van de experimentele opstelling. 


\section{Summary}

Hydrogels generally consist of a solid network and of a fluid permeating the network. Interactions between the solid and the fluid determine the response of a hydrogel when it is perturbed by, for example, the external mechanical pressure or the external osmotic pressure. The resulting time-dependent dynamics of (de)swelling are characterized by the poromechanical properties of hydrogels: their (visco)elasticity, plasticity and permeability. In this thesis we mainly focus on the characterization of the permeability and elasticity of hydrogels by modelling the dynamics of several (de)swelling processes for synthetic hydrogels and (bio)polymer gels. Also, we delve into the broader problem of compression tests on solids where we investigated a novel compression testing geometry, and we perform a poromechanical study of an industrially relevant phenomenon: the spontaneous expulsion of fluid from low fat mayonnaise.

In Chapter 2, we focus on the swelling and compression dynamics of synthetic hydrogels in (concentrated) polymer solutions. The hydrogels are observed to exhibit non-monotonic swelling and deswelling, an observation which asks for explanation. Through a numerically solved relaxational dynamics model, we give an accurate account of the measured volume of a hydrogel as a function of time. Through this account, we estimate the bulk modulus and the permeability of the hydrogel network, as well as the diffusion constant of the dissolved polymer molecules and the solvent quality change they bring about for the hydrogel network. On the other hand, through a poromechanical approach, we examine the theoretical response of a hydrogel on the diffusing polymers from a diluted solution, and we formulate an explicit expression for the displacement field of the hydrogel network and the concentration profile of polymer molecules in the hydrogel. Assuming the dominant contribution to the hydrogel dynamics of a diluted solution to also be the dominant response of a hydrogel 
in a concentrated solution, we construct a closed-form model for hydrogel dynamics in concentrated polymer solutions. Using this model, one can extract the bulk modulus, the permeability, the diffusion constant and the hydrogel-polymer interaction coefficient from volume measurements on a hydrogel.

In Chapter 3, we investigate a novel method to characterize the poromechanical properties of (bio)polymer gels and tissues from a ramp compression test in a commercial rheometer. As biopolymer gels, e.g., fibrin gels, are prone to stick to the rheometer plates during compression, we develop a novel approximate solution to the poroelastic equations of motion: a closed-form expression for the displacement field of the gel network and the flow field of the fluid where the gel is bonded to the rheometer plates. With this solution, the measured force in a ramp compression test can be interpreted to obtain the permeability and (effective) elastic properties of the gel network. Furthermore, we find our approximate solution, with appropriate phenomenological extensions, to be capable of probing the poromechanical properties of fibrin gels in the nonlinear regimes of large compression and strain stiffening. In particular, we find the contribution to the normal force in the linear regime to hold at large compression with a strain-dependent permeability, and we can model strain stiffening during compression with a stepwise increase in the shear modulus of the gel network.

In Chapter 4, we direct our attention to compression tests on solid materials. We consider a novel geometry in compression tests aiming to determine the Young's modulus of shape-limited materials and elasto-plastic materials which are sensitive to misalignment of the compression plates. In this compression geometry, where the upper plate is spherically tipped, we report the force-strain response of a linear elastic solid by solving the Navier-Cauchy equations through a novel perturbation approach.

Finally, in Chapter 5, we investigate the spontaneous expulsion of fluid, that is, syneresis, from an industrially relevant system: a model low fat mayonnaise. Considering the mayonnaise as a porous material, and by accounting for the geometry of the experimental setup with a two-cylinder model, we describe the measured expulsion and we critically evaluate the appropriateness of the experimental setup. 


\section{Dankwoord}

$\mathrm{Nu}$ is het tijd om dank te betuigen aan ieder die mij in staat heeft gesteld om dit proefschrift te voltooien. Allereerst gaat mijn dank uit naar God, de Schepper van hemel en aarde, van al wat zichtbaar en onzichtbaar is. $U$ hebt alles in het bestaan geroepen: alle onderwerpen die zijn onderzocht in dit proefschrift, mijzelf en alles wat mijn gebrekkige ik nodig had om dit werk te volbrengen. Vervolgens wil ik mijn ouders danken, door wiens onwankelbare liefde, zorg en opvoeding ik hier heb kunnen komen. Dan volgen mijn familie en vrienden: dank voor alle steun en interese, ook al is mijn onderzoek als een andere planeet voor de meesten van jullie. Verder dank aan alle collega's op AMOLF voor het goede (kantoor)gezelschap en wederom voor alle steun die ik heb mogen ontvangen, in het bijzonder toen ik kampte met pijnen vanwege het vele computerwerk.

Tenslotte een speciaal dankwoord gericht aan Bela, mijn promotor: allereerst wil ik je bedanken voor het vertrouwen dat je in mij hebt gesteld bij het vergeven van de promotieplek waarin ik dit proefschrift heb geschreven. Dankzij jouw vaderlijke bijstand heb ik deze promotie met succes kunnen voltooien. Je hebt mij gevormd in het (natuur)wetenschappelijke denken, ik ben er trots op in deze zin jouw zoon te zijn. Ik zie nu in dat de vereiste intellectuele zelfstandigheid in het wetenschappelijke bedrijf in het bijzonder door een promotie kan worden verkregen: hierbij moet je uit het nest worden gegooid opdat je kunt leren vliegen. Gelukkig was jij zo trouw om wekelijks mijn ideeën aan te horen en mij bij te sturen waar nodig.

In de toekomst hoop ik wetenschappelijk actief te blijven, al zij het niet meer in het puur natuurwetenschappelijke bedrijf. 


\section{References}

[1] J. F. Besseling and E. van der Giessen. Mathematical Modeling of Inelastic Deformation. Applied Mathematics. Taylor \& Francis, 1994, p. 328.

[2] S. R. Eugster. "On the Foundations of Continuum Mechanics and its Application to Beam Theories". PhD thesis. ETH Zurich, 2014, p. 122.

[3] H. D. Young et al. Sears and Zemansky's University Physics, 12th Edition. v. 1. Pearson Education, Limited, 2009, p. 1551.

[4] J. R. Taylor. Classical Mechanics. University Science Books, 2005, p. 786.

[5] C. Truesdell. "History of classical mechanics, part I". In: Die Naturwissenschaften 63.2 (1976), pp. 53-62.

[6] R. P. Agarwal and S. K. Sen. Creators of Mathematical and Computational Sciences. Springer International Publishing, 2014, p. 514.

[7] E. Feser. Aristotle's Revenge: The Metaphysical Foundations of Physical and Biological Science. Editiones scholasticae. Editiones Scholasticae, 2019, p. 515 .

[8] A. Driessen. "Aristotle and the Foundation of Quantum Mechanics". In: PhilSci-Archive [16265] (July 2019), p. 15.

[9] C. Rovelli. "Aristotle's Physics: A Physicist's Look". In: Journal of the American Philosophical Association 1.1 (Mar. 2015), pp. 23-40.

[10] O. Balaban. "The modern misunderstanding of Aristotle's theory of motion". In: Journal for General Philosophy of Science 26.1 (Mar. 1995), p. 10.

[11] R. Dugas. A History of Mechanics. London: Routledge \& Kegan Paul LTD, 1955, p. 661. 
[12] E. Mach and T. J. McCormack. The Science of Mechanics. Cambridge Library Collection - Physical Sciences. Cambridge University Press, 2013, p. 556.

[13] J. Sarnowsky. "Concepts of Impetus and the History of Mechanics". In: Mechanics and Natural Philosophy Before the Scientific Revolution. Ed. by W. R. Laird and S. Roux. Dordrecht: Springer Netherlands, 2008, pp. 121145 .

[14] M. Clagett. "Some General Aspects of Physics in the Middle Ages". In: Isis 39.1/2 (1948), pp. 29-44.

[15] P. Duhem. "Research on the history of physical theories". In: Synthese 83 (1990), pp. 189-200.

[16] S. Drake. "Impetus Theory Reappraised". In: Journal of the History of Ideas 36.1 (1975), pp. 27-46.

[17] J. L. Basdevant. Variational Principles in Physics. Springer New York, 2007, p. 186.

[18] D. Capecchi. History of Virtual Work Laws. Springer-Verlag Italia, 2012, p. 491.

[19] S. Timoshenko. History of Strength of Materials: With a Brief Account of the History of Theory of Elasticity and Theory of Structures. Dover Civil and Mechanical Engineering Series. Dover Publications, 1983, p. 452.

[20] G. A. Maugin. "On Porous Media and Mixtures". In: Continuum Mechanics through the Ages - From the Renaissance to the Twentieth Century. Springer, Cham, 2016. Chap. 3, pp. 57-79.

[21] J. E. Marsden and T. J. R. Hughes. Mathematical Foundations of Elasticity. Dover Civil and Mechanical Engineering Series. Dover, 1994, p. 576.

[22] G. A. Maugin. "Particles/Molecules Versus Continuum: The Never-Ending Debate". In: Continuum Mechanics through the Ages - From the Renaissance to the Twentieth Century. Springer, Cham, 2016. Chap. 1, pp. 1-25.

[23] R. de Boer. Theory of Porous Media. 1st ed. Springer-Verlag Berlin Heidelberg, 2000, p. 618.

[24] G. A. Maugin. Continuum Mechanics Through the Eighteenth and Nineteenth Centuries. Springer International Publishing, 2014, p. 275. 
[25] W. Ehlers. "Porous Media in the Light of History". In: The History of Theoretical, Material and Computational Mechanics - Mathematics Meets Mechanics and Engineering. Ed. by E. Stein. Berlin Heidelberg: SpringerVerlag, 2014. Chap. II, pp. 211-227.

[26] M. B. Clennell. "Tortuosity: a guide through the maze". In: Geological Society Special Publication 122 (1997), pp. 299-344.

[27] C. W. Macminn, E. R. Dufresne, and J. S. Wettlaufer. "Large Deformations of a Soft Porous Material”. In: Physical Review Applied 5.4 (2016), p. 30.

[28] O. Coussy. Poromechanics. Wiley, 2004, p. 298.

[29] B. E. Vos. "Fibrin structure and mechanics. A journey across scales". PhD thesis. VU University, 2018, p. 207.

[30] S. Whitaker. "Flow in Porous Media I: A theoretical derivation of Darcy's law". In: Transport in Porous Media 1 (1986), pp. 3-25.

[31] D. Lasseux and F. J. Valdés-Parada. "On the developments of Darcy's law to include inertial and slip effects". In: Comptes Rendus - Mecanique 345.9 (2017), pp. 660-669.

[32] D. Maity et al. "Response of collagen matrices under pressure and hydraulic resistance in hydrogels". In: Soft Matter 15.12 (2019), pp. 2617-2626.

[33] M. Doi. "Gel dynamics". In: Journal of the Physical Society of Japan 78.5 (2009), p. 19.

[34] M. Doi. "Onsager principle as a tool for approximation". In: Chinese Physics B 24.2 (2015), p. 6.

[35] M. Doi. "Onsager's Variational Principle in Soft Matter Dynamics". In: Nonequilibrium Soft Matter Physics. Ed. by S. Komura and T. Ōta. Series in soft condensed matter. World Scientific, 2012. Chap. 1, pp. 1-36.

[36] M. Doi. "Onsager's variational principle in soft matter". In: Journal of Physics Condensed Matter 23.28 (2011), p. 8.

[37] M. Doi and A. Onuki. "Dynamic coupling between stress and composition in polymer solutions and blends". In: Journal de Physique II 2.8 (1992), pp. 1631-1656. 
[38] M. Doi. "Effects of Viscoelasticity on Polymer Diffusion". In: Dynamics and patterns in complex fluids. Ed. by A. Ōnuki and K. Kawasaki. Springer proceedings in physics. Springer-Verlag, 1990, pp. 100-112.

[39] S. J. Buwalda et al. "Hydrogels in a historical perspective: From simple networks to smart materials". In: Journal of Controlled Release 190 (2014), pp. 254-273.

[40] F. Ganji, S. Vasheghani-Farahani, and E. Vasheghani-Farahani. "Theoretical Description of Hydrogel Swelling: A Review". In: Iranian Polymer Journal 19.5 (2010), pp. 375-398.

[41] J. M. van Bemmelen. "Das Hydrogel und das krystallinische Hydrat des Kupferoxyds". In: Zeitschrift für anorganische Chemie 5.1 (1894), pp. 466483.

[42] T. D. Campbell. "Synthesis and Physical Characterization of Biocompatible Hydrogels". PhD thesis. Florida State University, 2007, p. 99.

[43] P. J. Flory. "Molecular Size Distribution in Three Dimensional Polymers. I. Gelation". In: Journal of the American Chemical Society 63.11 (Nov. 1941), pp. 3083-3090.

[44] P. J. Flory. "Themodynamics of high polymer solutions". In: The Journal of Chemical Physics 10.1 (1942), pp. 51-61.

[45] M. L. Huggins. "Solutions of long chain compounds". In: The Journal of Chemical Physics 9.5 (1941), pp. 440-440.

[46] M. L. Huggins. "Properties of Rubber Solutions and Gels". In: Rubber Chemistry and Technology 16.3 (Sept. 1943), pp. 550-559.

[47] L. R. G. Treloar. "The thermo dynamic study of rubber-like elasticity". In: Proceedings of the Royal Society of London. Series B - Biological Sciences 139.897 (July 1952), pp. 506-512.

[48] L. R. G. Treloar. "The elasticity and related properties of rubbers". In: Reports on Progress in Physics 36.7 (July 1973), pp. 755-826.

[49] O. Wichterle and D. Lim. "Hydrophilic gels for biological use". In: Nature 185 (1960), pp. 117-118. 
[50] S. Thakur, V. K. Thakur, and O. A. Arotiba. "History, Classification, Properties and Application of Hydrogels: An Overview". In: Hydrogels - Recent Advances. Ed. by V. K. Thakur and M. K. Thakur. Singapore, 2018. Chap. 2, pp. 29-50.

[51] N. Chirani et al. "History and Applications of Hydrogels". In: Journal of Biomedical Sciencies 4.2 (2015), p. 23.

[52] K. A. Brandt, S. A. Goldman, and T. A. Inglin. Hydrogel-forming polymer compositions for use in absorbent structures. 1987.

[53] N. A. Peppas and L. Brannon-Peppas. "Controlled release of fragrances from polymers I. Thermodynamic analysis". In: Journal of Controlled Release 40.3 (July 1996), pp. 245-250.

[54] D. H. Lorenz. Skin adhesive hydrogel, its preparation and uses. 1992.

[55] K. Y. Lee and D. J. Mooney. "Hydrogels for Tissue Engineering". In: Chemical Reviews 101.7 (2001), pp. 1869-1880.

[56] M. S. Bae et al. "Photo-cured hyaluronic acid-based hydrogels containing growth and differentiation factor 5 (GDF-5) for bone tissue regeneration". In: Bone 59 (Feb. 2014), pp. 189-198.

[57] T. Wang et al. "Hydrogel sheets of chitosan, honey and gelatin as burn wound dressings". In: Carbohydrate Polymers 88.1 (Mar. 2012), pp. 75-83.

[58] G. W. Ashley et al. "Hydrogel drug delivery system with predictable and tunable drug release and degradation rates". In: Proceedings of the National Academy of Sciences of the United States of America 110.6 (2013), pp. 23182323.

[59] V. Nistor et al. "Stimuli-responsive cylindrical hydrogels mimic intestinal peristalsis to propel a solid object". In: Soft Matter 12.15 (Apr. 2016), pp. 3582-3588.

[60] S. A. Shahid et al. "Improvement in the Water Retention Characteristics of Sandy Loam Soil Using a Newly Synthesized Poly(acrylamide-co-acrylic Acid)/AlZnFe2O4 Superabsorbent Hydrogel Nanocomposite Material”. In: Molecules 17.8 (Aug. 2012), pp. 9397-9412.

[61] V. Arbona et al. "Hydrogel substrate amendment alleviates drought effects on young citrus plants". In: Plant and Soil 270.1 (Mar. 2005), pp. 73-82. 
[62] A. Rehab et al. "Controlled Release of Herbicides Supported on Polysaccharide Based Hydrogels". In: Journal of Bioactive and Compatible Polymers 6.1 (Jan. 1991), pp. 52-63.

[63] H. Mittal, S. S. Ray, and M. Okamoto. "Recent Progress on the Design and Applications of Polysaccharide-Based Graft Copolymer Hydrogels as Adsorbents for Wastewater Purification". In: Macromolecular Materials and Engineering 301.5 (May 2016), pp. 496-522.

[64] Y. C. Fung. Biomechanics: Mechanical Properties of Living Tissues. Biomechanics. Springer New York, 1993, p. 568.

[65] J. M. Wallace. "Skeletal Hard Tissue Biomechanics". In: Basic and Applied Bone Biology. 2nd ed. Academic Press, 2019. Chap. 7, pp. 125-140.

[66] W. F. Larrabee. "A finite element model of skin deformation. I. Biomechanics of skin and soft tissue: a review”. In: The Laryngoscope 96.4 (Apr. 1986), pp. 399-405.

[67] R. V. O'Toole et al. "Biomechanics for preoperative planning and surgical simulations in orthopaedics". In: Computers in Biology and Medicine 25.2 (Mar. 1995), pp. 183-191.

[68] A. Gefen, ed. Bioengineering Research of Chronic Wounds. Vol. 1. Studies in Mechanobiology, Tissue Engineering and Biomaterials. Berlin, Heidelberg: Springer Berlin Heidelberg, 2009, p. 449.

[69] M. Doblare and J. Merodio. Biomechanics. EOLSS Publications, 2015, p. 498.

[70] E. J. Gardner. History of Biology. 3rd ed. Mineapolis: Burgess Publishing Company, 1972, p. 464.

[71] M. Grene and D. J. Depew. "Aristotle and after". In: The philosophy of biology : an episodic history. Cambridge University Press, 2004. Chap. 1, pp. 134.

[72] B. Martin. "A Genealogy of Biomechanics”. In: Presidential Lecture. Pittsburgh, PA: 23rd Annual Conference of ASB, 1999.

[73] M. Ageno. "Methods and problems in biophysics". In: La Rivista del Nuovo Cimento 14.1 (Jan. 1991), p. 71. 
[74] P. Cranefield. "The Organic Physics of 1847 and the Biophysics of Today". In: Journal of the History of Medicine and Allied Sciences 12.4 (1957), pp. 407-423.

[75] W. Bialek. Biophysics: Searching for Principles. Princeton University Press, 2012.

[76] M. Barbieri. Introduction to Biosemiotics. Ed. by M. Barbieri. Dordrecht: Springer Netherlands, 2007.

[77] L. H. Hartwell et al. "From molecular to modular cell biology". In: Nature 402.December (1999), pp. 47-52.

[78] Yuri Lazebnik. "Can a biologist fix a radio?-Or, what I learned while studying apoptosis". In: Cancer Cell 67.6 (2002), pp. 179-182.

[79] U. Alon. "Network motifs: Theory and experimental approaches". In: Nature Reviews Genetics 8.6 (June 2007), pp. 450-461.

[80] M. Nakamura et al. "SPR2 protects minus ends to promote severing and reorientation of plant cortical microtubule arrays". In: Journal of Cell Biology 217.3 (Mar. 2018), pp. 915-927.

[81] B. Chakrabortty et al. "A Plausible Microtubule-Based Mechanism for Cell Division Orientation in Plant Embryogenesis". In: Current Biology 28.19 (Oct. 2018), pp. 3031-3043.

[82] E. E. Deinum et al. "How selective severing by katanin promotes order in the plant cortical microtubule array". In: Proceedings of the National Academy of Sciences of the United States of America 114.27 (July 2017), pp. 6942-6947.

[83] S. H. Tindemans, R. J. Hawkins, and B. M. Mulder. "Survival of the aligned: Ordering of the plant cortical microtubule array". In: Physical Review Letters 104.5 (Feb. 2010), p. 4.

[84] K. A. Jansen et al. "A guide to mechanobiology: Where biology and physics meet". In: Biochimica et Biophysica Acta (BBA) - Molecular Cell Research 1853.11 (Nov. 2015), pp. 3043-3052.

[85] E. Moeendarbary et al. "The cytoplasm of living cells behaves as a poroelastic material”. In: Nature Materials 12.3 (2013), pp. 253-261. 
[86] A. W. Holle et al. "Cell-Extracellular Matrix Mechanobiology: Forceful Tools and Emerging Needs for Basic and Translational Research". In: Nano Letters 18.1 (Jan. 2018), p. 8.

[87] K. A. Piez. "History of extracellular matrix: A personal view". In: Matrix Biology 16.3 (Aug. 1997), pp. 85-92.

[88] A. Serafini. The Epic History of Biology. Basic Books, 2001, p. 408.

[89] A. E. Ehret et al. "Inverse poroelasticity as a fundamental mechanism in biomechanics and mechanobiology". In: Nature Communications 8.1 (Dec. 2017), p. 10.

[90] C. Ghnatios et al. "Modeling soft, permeable matter with the proper generalized decomposition (PGD) approach, and verification by means of nanoindentation". In: Soft Matter 13.25 (2017), pp. 4482-4493.

[91] S. C. Cowin and L. Cardoso. "Mixture theory-based poroelasticity as a model of interstitial tissue growth”. In: Mechanics of Materials 44 (Jan. 2012), pp. 47-57.

[92] M. A. Swartz and M. E. Fleury. "Interstitial Flow and Its Effects in Soft Tissues". In: Annual Review of Biomedical Engineering 9.1 (2007), pp. 229256.

[93] R. de Boer. Trends in Continuum Mechanics of Porous Media. Dordrecht: Springer Netherlands, 2005, p. 282.

[94] J. W. Weisel and R. I. Litvinov. "Fibrin Formation, Structure and Properties". In: Fibrous Proteins: Structures and Mechanisms. Ed. by D. Parry and J. Squire. Vol. 82. Springer International Publishing, 2017, pp. 405-456.

[95] N. Laurens, P. Koolwijk, and M. P. M. de Maat. "Fibrin structure and wound healing". In: Journal of Thrombosis and Haemostasis 4.5 (May 2006), pp. 932-939.

[96] A. E. X. Brown et al. "Multiscale mechanics of fibrin polymer: Gel stretching with protein unfolding and loss of water". In: Science 325.5941 (2009), pp. 741-744.

[97] E. Ban et al. "Strong triaxial coupling and anomalous Poisson effect in collagen networks". In: Proceedings of the National Academy of Sciences 116.14 (2019), pp. 6790-6799. 
[98] J. Steinwachs et al. "Three-dimensional force microscopy of cells in biopolymer networks". In: Nature Methods 13.2 (2016), pp. 171-176.

[99] J. L. Shivers, S. Arzash, and F. C. MacKintosh. "Nonlinear Poisson effect governed by mechanical critical transition". In: arXiv:1905.09844v1 [condmat.soft] (2019), p. 7.

[100] C. Storm et al. "Nonlinear elasticity in biological gels". In: Nature 435.May (2005), pp. 191-194.

[101] P. K. Purohit et al. "Protein unfolding accounts for the unusual mechanical behavior of fibrin networks". In: Acta Biomaterialia 7.6 (June 2011), pp. 2374-2383.

[102] F. Esposito et al. "Fibrin sealants in dura sealing: A systematic literature review”. In: PLOS ONE 11.4 (Apr. 2016), p. 28.

[103] M. Radosevich, H. A. Goubran, and T. Burnouf. "Fibrin Sealant: Scientific Rationale, Production Methods, Properties, and Current Clinical Use”. In: Vox Sanguinis 72 (1997), pp. 133-143.

[104] P. Heher et al. "Fibrin-based delivery strategies for acute and chronic wound healing”. In: Advanced Drug Delivery Reviews 129 (Apr. 2018), pp. 134147.

[105] J. Gil et al. "A PEGylated fibrin hydrogel-based antimicrobial wound dressing controls infection without impeding wound healing". In: International Wound Journal 14.6 (Dec. 2017), pp. 1248-1257.

[106] O. Moreno-Arotzena et al. "Characterization of fibrin and collagen gels for engineering wound healing models". In: Materials 8.4 (2015), pp. 16361651.

[107] A. Verruijt. An Introduction to Soil Mechanics. Theory and Applications of Transport in Porous Media. Springer International Publishing, 2017, p. 420.

[108] V. Novák and H. Hlaváčiková. Applied Soil Hydrology. Theory and Applications of Transport in Porous Media. Springer International Publishing, 2018, p. 342.

[109] J. Bear and A. H. D. Cheng. Modeling Groundwater Flow and Contaminant Transport. Theory and Applications of Transport in Porous Media. Springer Netherlands, 2010, p. 756. 
[110] E. Fjar et al. Petroleum Related Rock Mechanics. Developments in Petroleum Science. Elsevier Science, 2008, p. 514.

[111] K. Khanafer et al. "Flow and Heat Transfer in Biological Tissues: Application of Porous Media Theory". In: Emerging Topics in Heat and Mass Transfer in Porous Media. Dordrecht: Springer Netherlands, 2008, pp. 237-259.

[112] R. de Boer. "Applications in Engineering and Biomechanics". In: Trends in Continuum Mechanics of Porous Media. Dordrecht: Springer Netherlands, 2005. Chap. 10, pp. 227-250.

[113] M. I. H. Khan et al. "Multiphase porous media modelling: A novel approach to predicting food processing performance". In: Critical Reviews in Food Science and Nutrition 58.4 (Mar. 2018), pp. 528-546.

[114] A. H. D. Cheng. Poroelasticity. Theory and Applications of Transport in Porous Media. Springer International Publishing, 2016, p. 877.

[115] Unilever. Hybrid soft materials: from physical mechanisms to designer products. Tech. rep. 2014, p. 49.

[116] K. A. Abbas et al. "Modified Starches and Their Usages in Selected Food Products: A Review Study". In: Journal of Agricultural Science 2.2 (2010), pp. 90-100.

[117] P. C. Hohenberg and B. I. Halperin. "Theory of dynamic critical phenomena". In: Reviews of Modern Physics 49.3 (1977), pp. 435-479.

[118] L. R. Kesselman et al. "Synthesis of monodisperse, covalently cross-linked, degradable "smart" microgels using microfluidics". In: Small 8.7 (2012), pp. 1092-1098.

[119] L. Ionov. "Hydrogel-based actuators: Possibilities and limitations". In: $M a$ terials Today 17.10 (2014), pp. 494-503.

[120] J. J. Sleeboom et al. "Compression and Reswelling of Microgel Particles after an Osmotic Shock". In: Physical Review Letters 119.9 (2017), p. 5.

[121] T. Tanaka and D. J. Fillmore. "Kinetics of swelling of gels". In: The Journal of Chemical Physics 70.3 (1979), pp. 1214-1218.

[122] H. M. Wyss et al. "Capillary micromechanics: Measuring the elasticity of microscopic soft objects”. In: Soft Matter 6.18 (2010), pp. 4550-4555. 
[123] C. Bonnet-Gonnet, L. Belloni, and B. Cabane. "Osmotic Pressure of Latex Dispersions”. In: Langmuir 10 (1994), pp. 4012-4021.

[124] K. Devanand and J. C. Selser. "Asymptotic Behavior and Long-Range Interactions in Aqueous Solutions of Poly(ethylene oxide)". In: Macromolecules 24.22 (1991), pp. 5943-5947.

[125] J. Tong and J. L. Anderson. "Partitioning and diffusion of proteins and linear polymers in polyacrylamide gels". In: Biophysical Journal 70.3 (1996), pp. 1505-1513.

[126] J. C. Williams, L. A. Mark, and S. Eichholtz. "Partition and Permeation of Dextran in Polyacrylamide Gel”. In: Biophysical Journal 75 (1998), pp. $493-$ 502 .

[127] H. O. Johansson et al. "Driving forces for phase separation and partitioning in aqueous two- phase systems". In: Journal of Chromatography B: Biomedical Applications 711.1-2 (1998), pp. 3-17.

[128] B. N. Preston et al. "Diffusion of dextran at intermediate concentrations". In: Journal of the Chemical Society, Faraday Transactions 1: Physical Chemistry in Condensed Phases 78.4 (Jan. 1982), pp. 1209-1221.

[129] Harries Research Group. Osmotic stress data PEG 20k, https://scholars.huji.ac.il/danielharries/book/peg-20k (accessed 12-122019).

[130] J. Crank. The Mathematics of Diffusion. Oxford science publications. Clarendon Press, 1979, p. 414.

[131] V. Klika. "A Guide through Available Mixture Theories for Applications". In: Critical Reviews in Solid State and Materials Sciences (2014), pp. 154174.

[132] R. L. Mauck, C. T. Hung, and G. A. Ateshian. "Modeling of Neutral Solute Transport in a Dynamically Loaded Porous Permeable Gel: Implications for Articular Cartilage Biosynthesis and Tissue Engineering”. In: Journal of Biomechanical Engineering (2003), pp. 602-614.

[133] S. Peppin, J. Elliott, and M. Worster. "Pressure and relative motion in colloidal suspensions". In: Physics of Fluids 17.5 (2005), p. 10. 
[134] T. M. Quinn and A. J. Grodzinsky. "Longitudinal modulus and hydraulic permeability of poly(methacrylic acid) gels: effects of charge density and solvent content". In: Macromolecules 26.16 (Aug. 1993), pp. 4332-4338.

[135] M. N. Ozisik. Boundary Value Problems of Heat Conduction. Dover Books on Engineering. Dover Publications, 2013, p. 512.

[136] T. Boudou et al. "An extended relationship for the characterization of Young's modulus and Poisson's ratio of tunable polyacrylamide gels". In: Biorheology 43 (2006), pp. 721-728.

[137] M. J. Krafcik and K. A. Erk. "Characterization of superabsorbent poly(sodium-acrylate acrylamide) hydrogels and influence of chemical structure on internally cured mortar". In: Materials and Structures 49.11 (2016), pp. 4765-4778.

[138] D. Sandrin et al. "Diffusion of macromolecules in a polymer hydrogel: From microscopic to macroscopic scales". In: Physical Chemistry Chemical Physics 18 (2016), pp. 12860-12876.

[139] P. A. Hassan, S. Rana, and G. Verma. "Making sense of Brownian motion: Colloid characterization by dynamic light scattering". In: Langmuir 31.1 (2015), pp. 3-12.

[140] S. W. Provencher. "A constrained regularization method for inverting data represented by linear algebraic or integral equations". In: Computer Physics Communications 27.3 (1982), pp. 213-227.

[141] J. Armstrong et al. "The Hydrodynamic Radii of Macromolecules and Their Effect on Red Blood Cell Aggregation”. In: Biophysical Journal 87.6 (Dec. 2004), pp. 4259-4270.

[142] S. M. Russell and G. Carta. "Mesh Size of Charged Polyacrylamide Hydrogels from Partitioning Measurements". In: Industrial \& Engineering Chemistry Research 44.22 (Oct. 2005), pp. 8213-8217.

[143] N. C. Stellwagen. "Apparent pore size of polyacrylamide gels: Comparison of gels cast and run in Tris-acetate-EDTA and Tris-borate-EDTA buffers". In: Electrophoresis 19.10 (July 1998), pp. 1542-1547.

[144] S. R. H. Davidson and M. D. Sherar. "Measurement of the thermal conductivity of polyacrylamide tissue-equivalent material". In: International Journal of Hyperthermia 19.5 (Jan. 2003), pp. 551-562. 
[145] A. Tél et al. "Heat conduction in poly(N-isopropylacrylamide) hydrogels". In: International Journal of Thermal Sciences 85 (Nov. 2014), pp. 47-53.

[146] M. Tokita and T. Tanaka. "Friction coefficient of polymer networks of gels". In: Journal of Chemical Physics 95.6 (1991), pp. 4613-4619.

[147] S. Caracciolo, B. M. Mognetti, and A. Pelissetto. "Virial coefficients and osmotic pressure in polymer solutions in good-solvent conditions". In: The Journal of Chemical Physics 125.9 (Sept. 2006), p. 8.

[148] R. K. Purama et al. "Structural analysis and properties of dextran produced by Leuconostoc mesenteroides NRRL B-640". In: Carbohydrate Polymers 76.1 (Mar. 2009), pp. 30-35.

[149] D. S. Clague and R. J. Phillips. "Hindered diffusion of spherical macromolecules through dilute fibrous media". In: Physics of Fluids 8.7 (July 1996), pp. 1720-1731.

[150] D. C. Lin, J. F. Douglas, and F. Horkay. "Development of minimal models of the elastic properties of flexible and stiff polymer networks with permanent and thermoreversible cross-links". In: Soft Matter 6.15 (July 2010), pp. 35483561 .

[151] R. H. Pritchard, Y. Y. Shery Huang, and E. M. Terentjev. "Mechanics of biological networks: From the cell cytoskeleton to connective tissue". In: Soft Matter 10.12 (2014), pp. 1864-1884.

[152] F. Huber et al. "Cytoskeletal crosstalk: When three different personalities team up". In: Current Opinion in Cell Biology 32 (2015), pp. 39-47.

[153] J. K. Mouw, G. Ou, and V. M. Weaver. "Extracellular matrix assembly: A multiscale deconstruction”. In: Nature Reviews Molecular Cell Biology 15.12 (2014), pp. 771-785.

[154] V. Vogel. "Unraveling the Mechanobiology of Extracellular Matrix". In: Annual Review of Physiology 80.1 (2018), pp. 353-387.

[155] Z. Bagoly et al. "Clot Structure and Fibrinolysis in Thrombosis and Hemostasis”. In: BioMed Research International (2017), p. 2.

[156] W. Wilson, C. C. van Donkelaar, and J. M. Huyghe. "A Comparison Between Mechano-Electrochemical and Biphasic Swelling Theories for Soft Hydrated Tissues". In: Journal of Biomechanical Engineering 127 (2005), pp. 158165. 
[157] A. Avendano, M. Cortes-Medina, and J. W. Song. "Application of 3-D Microfluidic Models for Studying Mass Transport Properties of the Tumor Interstitial Matrix”. In: Frontiers in Bioengineering and Biotechnology 7 (2019), p. 8 .

[158] G. T. Charras, T. J. Mitchison, and L. Mahadevan. "Animal cell hydraulics". In: Journal of Cell Science 122.18 (2009), pp. 3233-3241.

[159] R. S. Voronov et al. "Simulation of Intrathrombus Fluid and Solute Transport Using In Vivo Clot Structures with Single Platelet Resolution”. In: Annals of Biomedical Engineering 41.6 (2013), pp. 1297-1307.

[160] Z. Chen et al. "Thrombus Permeability on Dynamic CTA Predicts Good Outcome after Reperfusion Therapy". In: American Journal of Neuroradiology 39.10 (2018), pp. 1854-1859.

[161] E. M. Santos et al. "Thrombus Permeability Is Associated with Improved Functional Outcome and Recanalization in Patients with Ischemic Stroke". In: Stroke 47.3 (2016), pp. 732-741.

[162] M. Pieters et al. "An international study on the standardization of fibrin clot permeability measurement: Methodological considerations and implications for healthy control values". In: Journal of Thrombosis and Haemostasis 10.10 (Oct. 2012), pp. 2179-2181.

[163] R. E. Shadwick. "Mechanical design in arteries". In: The Journal of experimental biology 202 (1999), pp. 3305-3313.

[164] G. S. Schultz, G. Ladwig, and A. Wysocki. "Extracellular matrix: review of its roles in acute and chronic wounds". In: World Wide Wounds (2010), p. 8.

[165] F. Burla et al. "From mechanical resilience to active material properties in biopolymer networks". In: Nature Reviews Physics 1.4 (2019), pp. 249-263.

[166] B. A. Roeder. "Fibril Microstructure Affects Strain Transmission Within Collagen Extracellular Matrices". In: Journal of Biomechanical Engineering 131.3 (2009), p. 11.

[167] A. S. G. van Oosten et al. "Uncoupling shear and uniaxial elastic moduli of semiflexible biopolymer networks: compression-softening and stretchstiffening”. In: Scientific Reports 6.1 (2016), p. 9.

[168] O. V. Kim et al. "Structural basis for the nonlinear mechanics of fibrin networks under compression”. In: Biomaterials 35.25 (2014), pp. 6739-6749. 
[169] O. V. Kim et al. "Foam-like compression behavior of fibrin networks". In: Biomechanics and Modeling in Mechanobiology 15.1 (2016), pp. 213-228.

[170] T. Brynk et al. "Experimental poromechanics of trabecular bone strength: Role of Terzaghi's effective stress and of tissue level stress fluctuations". In: Journal of Biomechanics 44.3 (2011), pp. 501-508.

[171] L. Cardoso et al. "Advances in assessment of bone porosity, permeability and interstitial fluid flow”. In: Journal of Biomechanics 46.2 (2013), pp. 253-265.

[172] H. C. De Cagny et al. "Porosity governs normal stresses in polymer gels". In: Physical Review Letters 117.21 (2016), p. 5.

[173] C. Yeromonahos, B. Polack, and F. Caton. "Nanostructure of the fibrin clot". In: Biophysical Journal 99.7 (2010), pp. 2018-2027.

[174] M. D. Bale, M. F. Mullert, and J. D. Ferry. "Effects of fibrinogen-binding tetrapeptides on mechanical properties of fine fibrin clots (modulus of elasticity/viscoelastic creep/peptides)". In: Biochemistry 82.5 (1985), pp. 14101413.

[175] F. L. Macrae et al. "A fibrin biofilm covers the blood clot and protects from microbial invasion”. In: The Journal of Clinical Investigation 128.8 (2018), pp. 3356-3368.

[176] C. G. Armstrong, W. M. Lai, and V. C. Mow. "An Analysis of the Unconfined Compression of Articular Cartilage". In: Journal of Biomechanical Engineering 106.2 (1984), pp. 165-173.

[177] S. Qiao and N. Lu. "Analytical solutions for bonded elastically compressible layers". In: International Journal of Solids and Structures 58 (2015), pp. 353365 .

[178] I. K. Piechocka et al. "Structural hierarchy governs fibrin gel mechanics". In: Biophysical Journal 98.10 (2010), pp. 2273-2280.

[179] S. Toll. "Packing mechanics of fiber reinforcements". In: Polymer Engineering and Science 38.8 (1998), pp. 1337-1350.

[180] J. V. Shah and P. A. Janmey. "Strain hardening of fibrin gels and plasma clots”. In: Rheologica Acta 36.3 (1997), pp. 262-268. 
[181] T. Yamaue and M. Doi. "Theory of one-dimensional swelling dynamics of polymer gels under mechanical constraint". In: Physical Review E - Statistical Physics, Plasmas, Fluids, and Related Interdisciplinary Topics 69.4 (2004), p. 5.

[182] N. A. Kurniawan et al. "Fibrin Networks Support Recurring Mechanical Loads by Adapting their Structure across Multiple Scales”. In: Biophysical Journal 111.5 (2016), pp. 1026-1034.

[183] J. W. Weisel and C. Nagaswami. "Computer modeling of fibrin polymerization kinetics correlated with electron microscope and turbidity observations: clot structure and assembly are kinetically controlled". In: Biophysical Journal 63 (1992), pp. 111-128.

[184] J.-P. Collet et al. "The elasticity of an individual fibrin fiber in a clot". In: Proceedings of the National Academy of Sciences 102.26 (2005), pp. 91339137.

[185] M. E. Carr and J. Hermans. "Size and Density of Fibrin Fibers from Turbidity”. In: Macromolecules 11.1 (1978), pp. 46-50.

[186] ASTM International. "Standard test methods of compression testing of metallic materials at room temperature". In: Annual Book of ASTM Standards. Vol. 03.01. West Conshohocken, PA: ASTM International, 2000. Chap. ASTM-E9-89, p. 9.

[187] ASTM International. "Standard Test Method for Compressive Properties of Rigid Plastics". In: Annual Book of ASTM Standards. Vol. 08.01. West Conshohocken, PA: ASTM International, 2015, p. 8.

[188] M. N. Charalambides et al. "The analysis of the frictional effect on stress strain data from uniaxial compression of cheese". In: Journal of Materials Science 36.9 (2001), pp. 2313-2321.

[189] M. N. Charalambides et al. "Effect of friction on uniaxial compression of bread dough". In: Journal of Materials Science 40.13 (2005), pp. 3375-3381.

[190] M. L. Anderson, P. H. Mott, and C. M. Roland. "The compression of bonded rubber disks". In: Rubber Chem. technol. 77.3 (2004), pp. 293-302. 
[191] J. G. Williams and C. Gamonpilas. "Using the simple compression test to determine Young's modulus, Poisson's ratio and the Coulomb friction coefficient". In: International Journal of Solids and Structures 45.16 (2008), pp. 4448-4459.

[192] K. Chau and S. Watanabe. "Young's modulus interpreted from compression tests with end friction". In: Journal of Engineering Mechanics ASCE 123.1 (1997), p. 7.

[193] J. R. Barber. "End Effects". In: Elasticity. Dordrecht: Springer Netherlands, 2010, pp. 77-89.

[194] W. E. Luecke et al. Repeatability and reproducibility of compression strength measurements conducted according to ASTM E9. Tech. rep. National Institute of Standards and Technology, U.S. Department of Commerce, 2009, p. 39.

[195] J. Dong et al. "The Influence of Boundary Conditions on Modulus Measurement in Uniaxial Compression Tests". In: Experimental Techniques 41.3 (2017), pp. 327-330.

[196] R. Knops and L. Payne. Uniqueness Theorems in Linear Elasticity. 1st ed. Springer-Verlag Berlin Heidelberg, 1971, p. 132.

[197] E. J. Hinch, D. G. Crighton, and M. J. Ablowitz. Perturbation Methods. Cambridge Texts in Applied Mathematics. Cambridge University Press, 1991, p. 160.

[198] J. H. Bramble and L. E. Payne. "Some uniqueness theorems in the theory of elasticity”. In: Archive for Rational Mechanics and Analysis 9 (Jan. 1962), pp. 319-328.

[199] P. Wang. "The limiting case of zero shear modulus in linear elasticity". In: Journal of Elasticity 38.2 (Feb. 1995), pp. 121-132.

[200] E. Radi, E. Dragoni, and A. Spaggiari. "Adhesively bonded disk under compressive diametrical load". In: International Journal of Solids and Structures 152-153 (2018), pp. 51-65.

[201] J. R. Barber. "Three-dimensional elasticity problems for the prismatic bar". In: Proceedings of the Royal Society A: Mathematical, Physical and Engineering Sciences 462.2070 (2006), pp. 1877-1896. 
[202] B. Brady. "An exact solution to the radially end-constrained circular cylinder under triaxial loading". In: International Journal of Rock Mechanics and Mining Sciences \& Geomechanics Abstracts 8.2 (1971), pp. 165-178.

[203] S. Moghe and H. Neff. "Elastic Deformations of Constrained Cylinders". In: Journal of Applied Mechanics 38 (1971), pp. 393-399.

[204] J. R. Barber. Elasticity. Solid Mechanics and Its Applications. Springer Netherlands, 2010, p. 534.

[205] S. P. Timoshenko and J. N. Goodier. Theory of elasticity. 3rd ed. Engineering societies monographs. McGraw-Hill, 1987, p. 567.

[206] M. J. Miles et al. "The roles of amylose and amylopectin in the gelation and retrogradation of starch". In: Carbohydrate Research 135.2 (1985), pp. 271281.

[207] V. J. Morris. "Starch gelation and retrogradation". In: Trends in Food Science and Technology 1.C (1990), pp. 2-6.

[208] R. D. Kamien and A. J. Liu. "Why is random close packing reproducible?" In: Physical Review Letters 99.15 (2007), p. 4.

[209] K. Miyazaki et al. "Nonlinear viscoelasticity of metastable complex fluids". In: Europhysics Letters 75.6 (2006), pp. 915-921.

[210] T. B. Blijdenstein et al. "Scaling behavior of delayed demixing, rheology, and microstructure of emulsions flocculated by depletion and bridging". In: Langmuir 20.26 (2004), pp. 11321-11328.

[211] A. H. Krall, Z. Huang, and D. A. Weitz. "Dynamics of density fluctuations in colloidal gels". In: Physica A: Statistical Mechanics and its Applications 235.1-2 (1997), pp. 19-33.

[212] A. Meller et al. "Viscoelasticity of depletion-induced gels in emulsionpolymer systems”. In: Langmuir 15.6 (1999), pp. 1918-1922.

[213] T. Gibaud, D. Frelat, and S. Manneville. "Heterogeneous yielding dynamics in a colloidal gel”. In: Soft Matter 6.15 (2010), pp. 3482-3488.

[214] T. Gibaud et al. "Multiple yielding processes in a colloidal gel under large amplitude oscillatory stress”. In: Soft Matter 12.6 (2016), pp. 1701-1712.

[215] A. H. Krall and D. A. Weitz. "Internal Dynamics and Elasticity of Fractal Colloidal Gels”. In: Physical Review Letters 80.4 (1998), pp. 778-781. 
[216] J. M. Van Doorn et al. "Linking Particle Dynamics to Local Connectivity in Colloidal Gels”. In: Physical Review Letters 118.18 (2017), p. 5.

[217] S. Wang et al. "Starch Retrogradation: A Comprehensive Review". In: Comprehensive Reviews in Food Science and Food Safety 14.5 (2015), pp. 568585.

[218] B. A. Westrin, A. Axelsson, and G. Zacchi. "Diffusion measurement in gels". In: Journal of Controlled Release 30.3 (July 1994), pp. 189-199.

[219] J. P. Gong et al. "Synthesis of Hydrogels with Extremely Low Surface Friction". In: Journal of the American Chemical Society 123.23 (June 2001), pp. 5582-5583.

[220] V. Arbabi et al. "Determination of the mechanical and physical properties of cartilage by coupling poroelastic-based finite element models of indentation with artificial neural networks". In: Journal of Biomechanics 49.5 (Mar. 2016), pp. 631-637.

[221] Y. Hu et al. "Using indentation to characterize the poroelasticity of gels". In: Applied Physics Letters 96.12 (Mar. 2010), p. 3.

[222] K. Miller. "Method of testing very soft biological tissues in compression". In: Journal of Biomechanics 38 (Jan. 2005), pp. 153-158.

[223] V. V. Mozhaev et al. "High pressure effects on protein structure and function". In: Proteins: Structure, Function, and Genetics 24.1 (Jan. 1996), pp. 81-91.

[224] A. L. Fogelson and J. P. Keener. "Toward an understanding of fibrin branching structure". In: Physical Review E 81.5 (May 2010), p. 9.

[225] J. Gibbings. Dimensional Analysis. London: Springer London, 2011, p. 297.

[226] P. W. Bridgman. Dimensional Analysis. New Haven: Yale University Press, 1963.

[227] G. I. Barenblatt et al. Scaling, Self-similarity, and Intermediate Asymptotics: Dimensional Analysis and Intermediate Asymptotics. Cambridge Texts in Applied Mathematics. Cambridge University Press, 1996, p. 386.

[228] M. Punter et al. "Poroelasticity of (bio)polymer networks during compression: theory and experiment”. In: Soft Matter Accepted Manuscript (2020), p. 8. 
[229] Q. Wu et al. "Gravity-driven syneresis in model low-fat mayonnaise". In: Soft Matter 15.46 (Nov. 2019), pp. 9474-9481.

[230] M. Punter et al. "Self-Assembly Dynamics of Linear Virus-Like Particles: Theory and Experiment". In: Journal of Physical Chemistry B 120.26 (2016), pp. 6286-6297.

[231] J. C. Everts et al. "A Landau-de Gennes theory for hard colloidal rods: Defects and tactoids". In: Journal of Chemical Physics 144.19 (2016), p. 13. 


\section{About the author}

Melle Punter (1990) was born in Amsterdam, the Netherlands. After finishing the Atheneum studies at Hofstad Lyceum (formerly known as St-Janscollege) in The Hague (2008), while visiting Pre-University College at Leiden University, he tasted of the newly established Future Planet Studies at the University of Amsterdam for one year. Afterwards, he switched to study physics at the University of Utrecht, obtaining a Bachelor of Science (2012) with a thesis on Landau-de Gennes theory for lyotropic liquid crystals under the supervision of Prof. Dr René van Roij and Prof. Dr Paul van der Schoot. In a gap year he served in the board of the Catholic Student Parish of Utrecht (KSU), after which he earned a Master of Science in Theoretical Physics (2015). He performed master thesis research under the supervision of Prof. Paul van der Schoot on the role of assembly signals in the self-assembly of linear virus-like particles. After his physics studies he conducted research into the mechanics and syneresis of gels under the supervision of Prof. Dr Bela Mulder, the thesis of which is presented in this book.

\section{Peer-reviewed journal publications}

- M. Punter et al. "Poroelasticity of (bio)polymer networks during compression: theory and experiment". In: Soft Matter Accepted Manuscript (2020), p. 8.

- Q. Wu et al. "Gravity-driven syneresis in model low-fat mayonnaise". In: Soft Matter 15.46 (Nov. 2019), pp. 9474-9481.

- J. J. Sleeboom et al. "Compression and Reswelling of Microgel Particles after an Osmotic Shock”. In: Physical Review Letters 119.9 (2017), p. 5.

- M. Punter et al. "Self-Assembly Dynamics of Linear Virus-Like Particles: Theory and Experiment". In: Journal of Physical Chemistry B 120.26 (2016), pp. 6286-6297.

- J. C. Everts et al. "A Landau-de Gennes theory for hard colloidal rods: Defects and tactoids". In: Journal of Chemical Physics 144.19 (2016), p. 13. 


\section{Overview of completed training activities}

With the training and education activities listed below the $\mathrm{PhD}$ candidate has complied with the requirements set by the VLAG Research School which comprises of a minimum total of 30 ECTS (=21 weeks of activities)

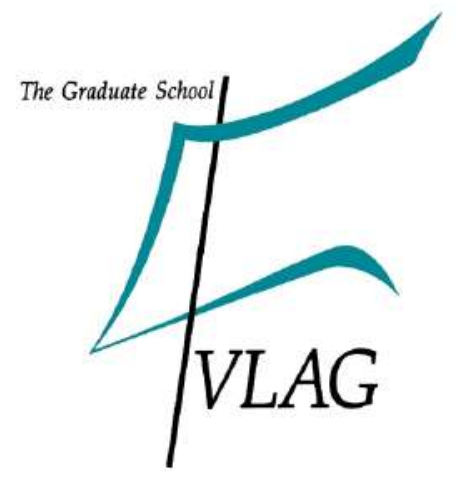

\section{Discipline specific activities (12.4 ECTS)}

- Han-sur-Lesse winterschool (WUR/TU-Delft, 2016)

- $6^{\text {th }}$ Biot conference (ECIS, 2017)

- Interpore (TU-Delft, 2017)

- Biophysics@ Veldhoven (NWO; 2015,2016)

-Physics@ Veldhoven (NWO; 2017,2018,2019)

- Biannual Soft Matter Meeting (WUR/UvA/UU/UT/TUE, 2015-2019)

- FYSICA (NNV; 2017,2018)

\section{General courses (6.3 ECTS)}

- Promotie in eigen regie (FOM, 2015)

- Young Scientists' Day (FOM/NWO; 2015,2017)

- Art of presenting science (FOM, 2016)

- Writing better code (FOM, 2016)

- Physics with Industry (Lorentz Center, 2017)

- Career orientation taster day (Cygnus Gymnasium Amsterdam, 2018)

\section{Optional courses and activities (12.1 ECTS)}

- AMOLF institute colloquium (AMOLF, 2015-2019)

- Theory and Computation meeting (AMOLF, 2015-2019)

- Biomeeting presentations (AMOLF, 2015-2019)

- Biomeeting poster session (AMOLF, 2015-2019)

- Theory of Biomolecular Matter group meeting (AMOLF, 2016-2019) 
The research described in this thesis has been performed at the NWO Institute AMOLF, Science Park 104, 1098 XG Amsterdam, The Netherlands.

This work is part of an Industrial Partnership Programme of the Foundation for Fundamental Research on Matter (FOM), which is part of the Netherlands Organisation for Scientific Research (NWO), and the NWO division Earth and Life Sciences. This Industrial Partnership Programme is co-financed by Unilever Research and Development B.V. 